\title{
ÜBERLEGUNGEN ZU DEN THEOGNIDEEN
}

\author{
Thomas Gärtner
}

\begin{abstract}
The following remarks concerning textual criticism are not intended to extrapolate from the Byzantine redactions any kind of "Ur-Theognis“, i.e. to reconstruct archaic elegies, from which the transmitted verses might have arisen by being repeatedly excerpted. Rather they start from the fact that the individual pieces as presented in West's editions are on the one hand text segments of high and concise stylistic quality, while on the other they have become heavily corrupt. Corrupt pieces of text may be recognized and emended by the process of text-immanent observation. Consequently immanent textual criticism without recurrence to an „Ur-Theognis“ seems to be possible.
\end{abstract}

\section{a. Textkritik}

Die folgenden textkritischen Bemerkungen zielen nicht darauf ab, aus den byzantinischer Redaktion entstammenden "Theognideen“ einen "Ur-Theognis" herauszuschälen, d.h. archaische Elegien zu rekonstruieren, aus denen auf dem Wege mehrfachen Exzerpierens die vorliegenden „Theognideen“ entstanden sein könnten. Sie gehen vielmehr von der Beobachtung aus, daß in den Einzelstücken, wie sie etwa in Wests modernen Ausgaben vorliegen, Textsegmente von hoher sprachlicher Qualität und Prägnanz vorliegen, die oftmals von schweren Korruptelen betroffen sind, und daß sich solche Korruptelen in der Regel mit Hilfe textimmanenter, d.h. nicht über das jeweilige Textsegment herausreichender, sprachlicher Beobachtungen emendieren lassen, daß also eine „immanente“ Textkritik der Einzelexzerpte ohne hypothetischen Rekurs auf eine zu rekonstruierende „Urelegie“ möglich ist. Wenn im folgenden von einer "Urfassung“ die Rede ist, geht es nur um die von Korruptelen freie Gestalt eines solchen Textsegments, nicht etwa um eine „Urelegie“.

\section{(1) Cruces innerhalb der Theognideen}

So existiert eine große Zahl anerkannter Cruces innerhalb der Theognideen, deren Heilung nicht aussichtslos erscheint.

$125-128$

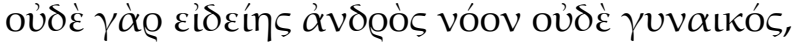

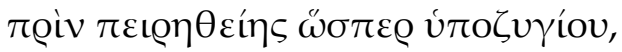

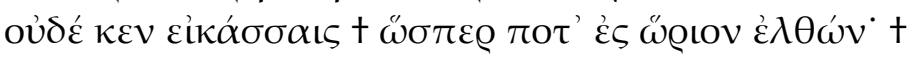

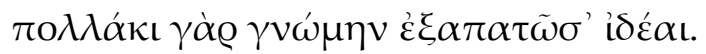

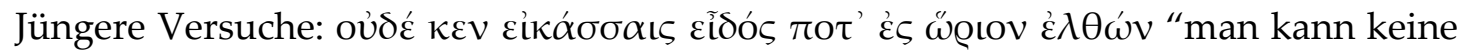
Mutmaßungen anstellen, wenn man auf jemand trifft, der ein schönes Äußeres hat"

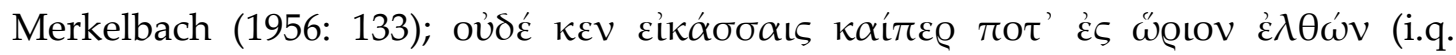




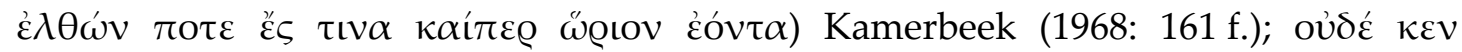

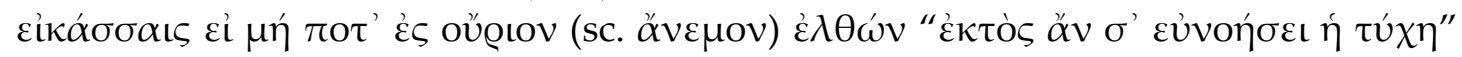

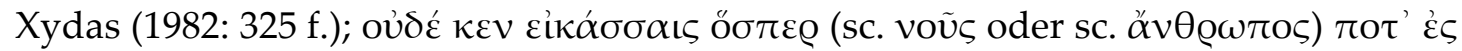
$\alpha$ ụ́ıov ع̌ $\lambda \theta$ oı "né potresti immaginarti come possa presentarsi domani" Ercolani

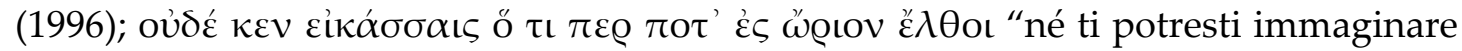

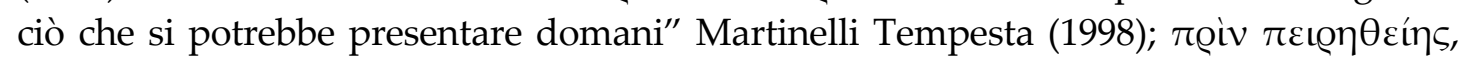

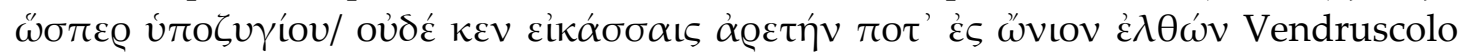

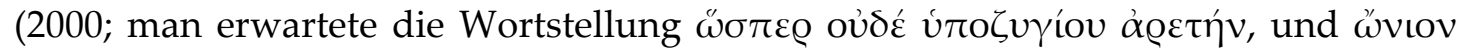
i.q. "Verkaufsplatz" ist nicht belegt, s.u.). Gerber (1970: 281) deutet die Überlieferung

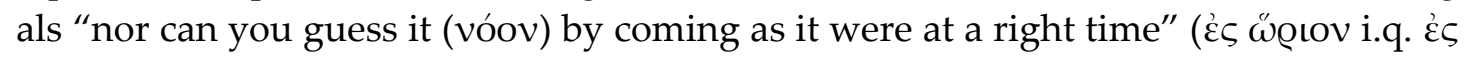

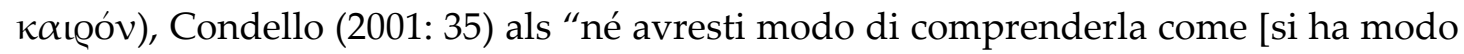
di comprenderla] quando si giunge a un momento cruciale".

West notiert im Apparat von den modernen Versuchen, wohl zurecht, nur die Konjektur des Camerarius, ف̈vเov. Insofern dieses Wort nur ein Handelsgut, nicht einen Handelsplatz bezeichnen kann, muß dann die Praeposition $\dot{\varepsilon} \varsigma$ korrupt sein.

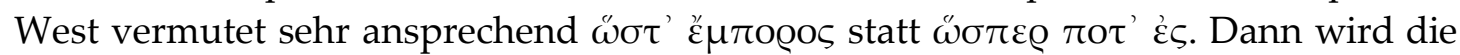
Person, die ihre Mitmenschen bewertet, verglichen mit einem Handelsgüter begutachtenden Kaufmann; so würde der Vergleich $\omega \sigma \pi \varepsilon \varrho$ vं vorigen Vers passend fortgeführt. Am Versende vermutet man ein transitives

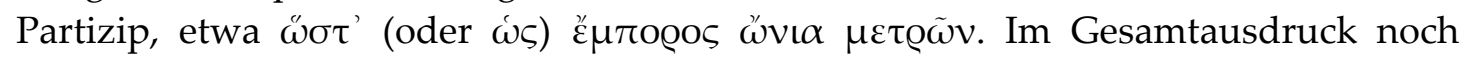

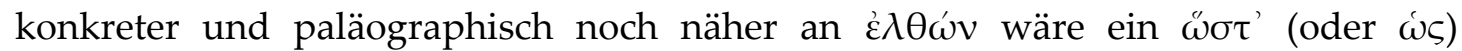

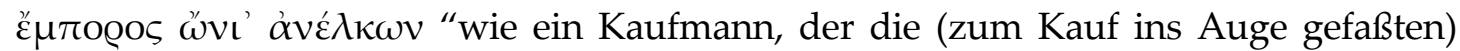
Waren (wägend) emporzieht". Zur Verwendung von ḋvé $\kappa \kappa \omega v$ vgl. Hom. Il. M 433-

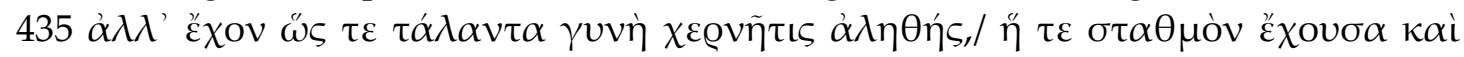

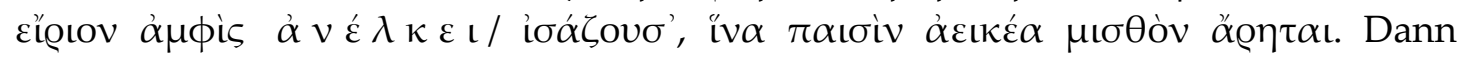

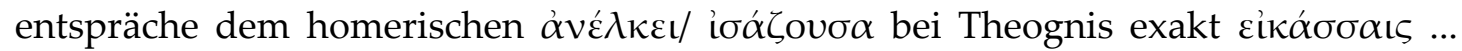

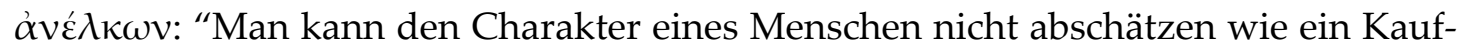
mann seine Ware durch (wägendes) Hochziehen". Zum unvermittelten Gebrauch der Wägemetapher "Ziehen" im gleichen Zusammenhang in den Theognideen vgl.

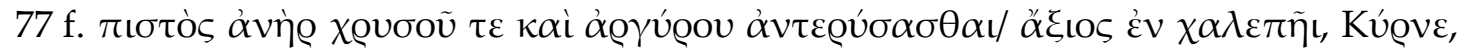

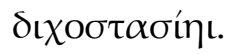

$1097-1100$

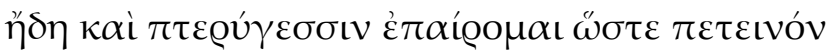

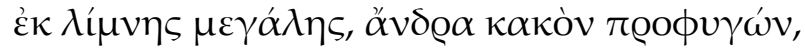

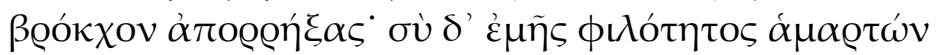

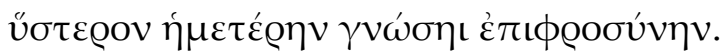

Die in Wests Apparat notierten Konjekturen zu Vers 1098 ( $\lambda$ ó $\chi \mu\rceil$ s Hermann, ihm

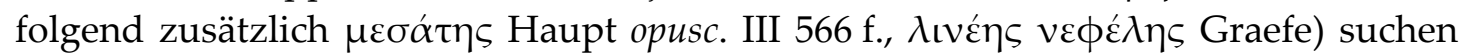
die Vogelmetaphorik aus Vers 1097 fortzuführen. Dies ist jedoch kaum erforderlich.

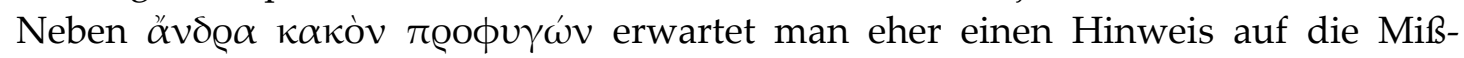
handlung, welcher der Sprecher entkommen ist, wohl im Zusammenhang einer

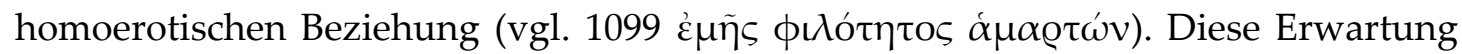

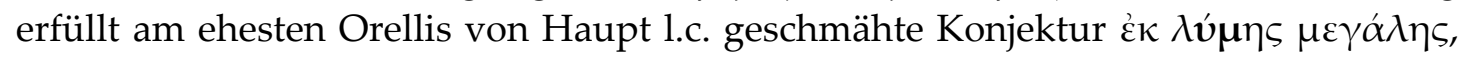

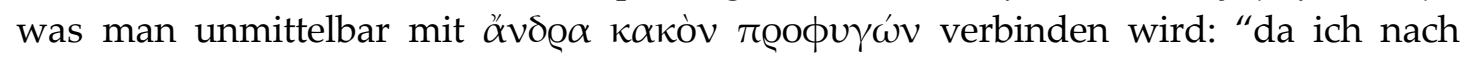


schwerer Mißhandlung einem üblen Mann entkommen bin". So wird der Text richtig nach Orelli hergestellt und verstanden bei van Herwerden (1870: 41), der mit Hilfe

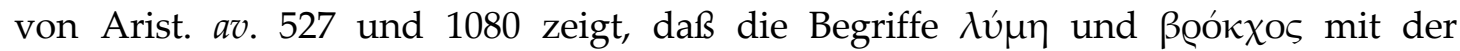
Vorstellung eines gefangenen Vogels durchaus vereinbar sind: "ceu avis, quae rupto laqueo aucupem contumeliosamque sortem effugit, ego te effugi".

$1197-1202$

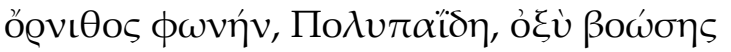

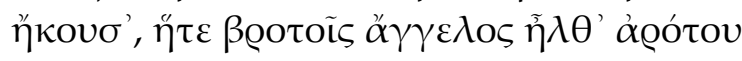

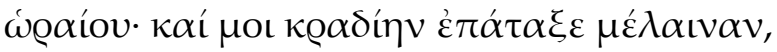

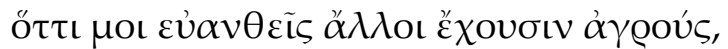

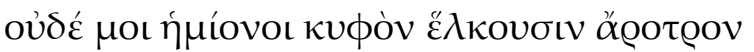

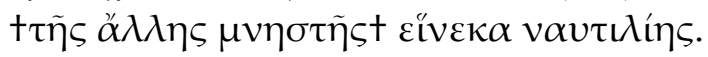

Der Versuch Nagys (1982: 109-111), die Überlieferung mit Parallelen aus Hesiod zu rechtfertigen und insbesondere $\alpha \ddot{\alpha} \lambda \lambda\rceil \varsigma$ in dem Sinne "unseasonal" bzw. "dangerous" (vgl. Gerber 1991: 198) zu deuten, schlägt nicht durch.

West (1974: 164 f.) verwirft alle früheren Konjekturen und berücksichtigt in

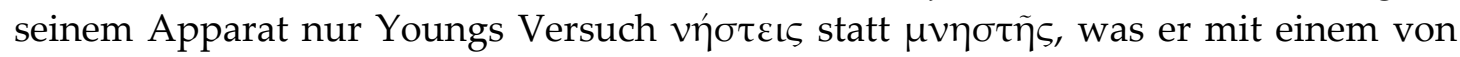

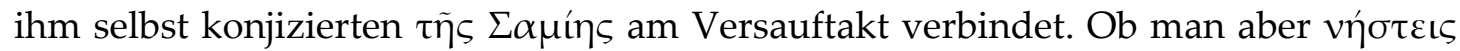
mit West 1.c. im Sinne von Hes. op. 459-461 von rastlosem Arbeiten der Tiere infolge des durch eine Seereise bedingten verspäteten Beginns des Pflügens verstehen kann, ist zweifelhaft: Denn wenn das dichterische Ich zu Beginn der Pflügezeit Schmerz

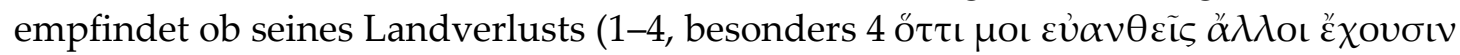
$\dot{\alpha} \gamma$ oov́s), so ist es wahrscheinlicher, daß für ihn überhaupt keine Halbesel pflügen als daß diese besonders angestrengt ( (was ja voraussetzen würde, daß Ackerland noch vorhanden wäre). Gerber sagt zurecht: "It seems likely that Theognis has lost his land because of a $\mathrm{d}$ i s a s t r o u s s e a v o y a g e "; ebenso erkennt Erbse (1998: 240), daß ein "abfälliges Urteil über die Seefahrt" erfordert ist - dieser Gedanke kommt in seiner eigenen Konjektur $\tau \tilde{\eta} \varsigma$

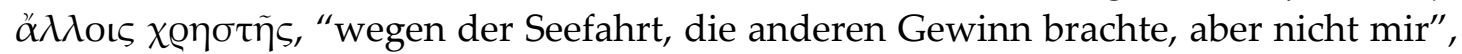
nur sehr indirekt heraus ${ }^{1}$. Genau diesen Gedanken erwartet man am Schlusse des

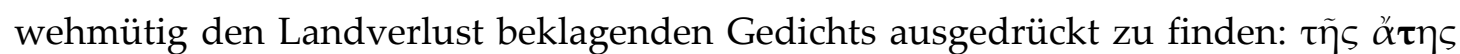

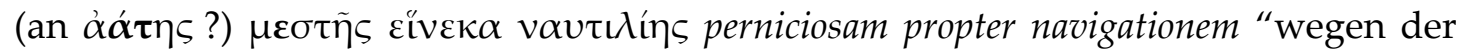

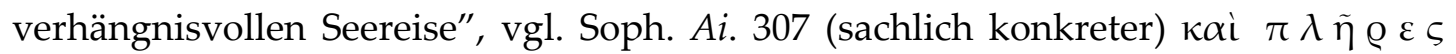

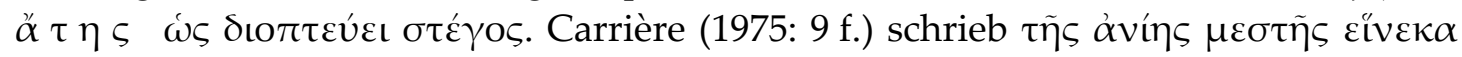

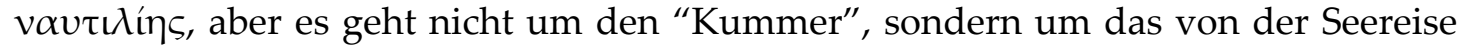
angerichtete, in den ersten beiden Distichen in seinen subjektiven Folgen ausgemalte "Verderben".

$1279-1282$

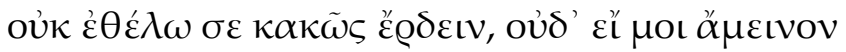

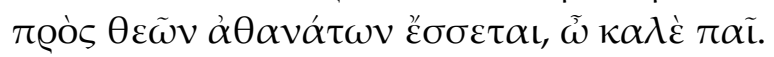

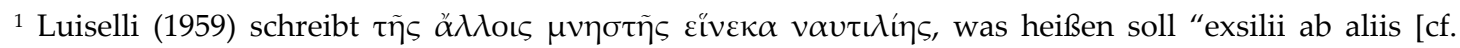

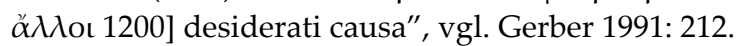




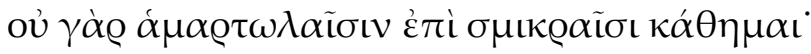

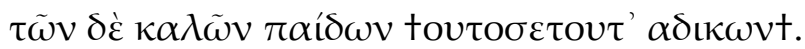

Sämtlichen anderen konjekturalen Versuchen entschieden vorzuziehen ist Boissona-

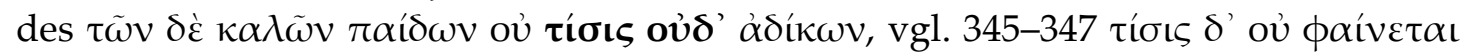

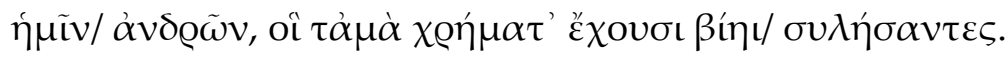

$1305-1310$

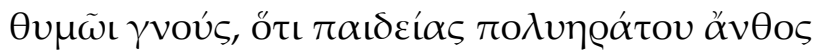

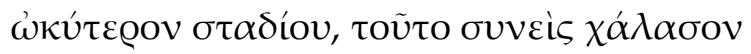

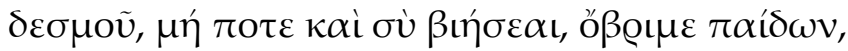

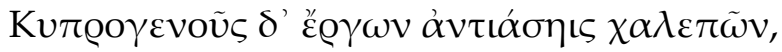

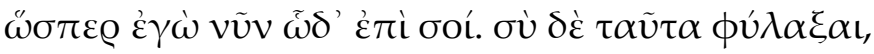

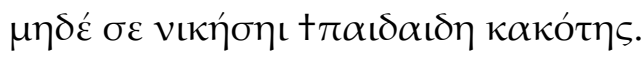

Die Emendation des Schlußverses liegt wesentlich näher als die in Wests Apparat

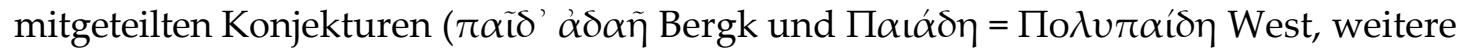
Vorschläge bei Vetta). Die Schlußwarnung kann doch kaum darauf gehen, daß der angesprochene Knabe von moralischer Schlechtigkeit "besiegt", d.h. überkommen wird (was aus Sicht des Sprechers längst eingetreten sein müßte). Vielmehr wird er

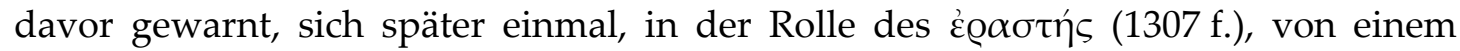
Knaben ähnlicher Charakteranlage, wie er selbst sie jetzt offenbart, "besiegen zu

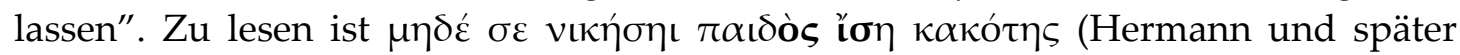
Kroll 1955: 79). Zur Kurzmessung der ersten Silbe von loos vgl. 678 und zu 183-192.

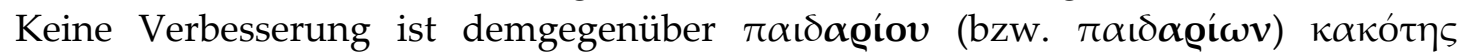
(Garzya 1957: 217), weil mit dieser Konjektur der in ı́o $\eta$ liegende Aspekt der Vergleichbarkeit der Situationen nicht zum Ausdruck kommt.

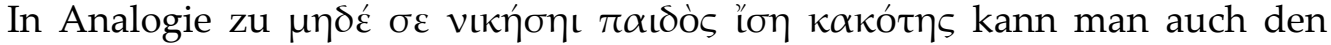
sonderbaren und unpassenden - denn in der ausgemalten Situation ist der Angeredete kein "Knabe" mehr, sondern bereits seinerseits غ̇ $\alpha \sigma \tau \eta \dot{s}$ - Vokativ

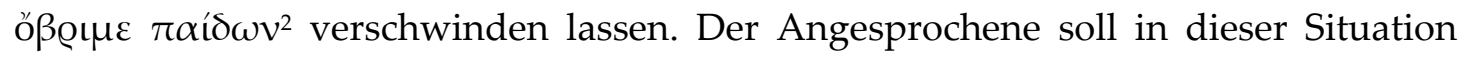
keine Gewalt durch (ähnliche) Übermütigkeiten von Knaben erleiden: $\mu \eta \dot{\eta} \pi о \tau \varepsilon \kappa \alpha i$

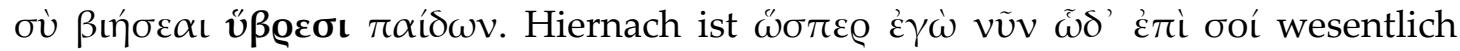
ausdruckskräftiger. Der Plural von ưß@ı ist schon belegt bei Hes. op. $145 \mathrm{f}$. oĩoเv

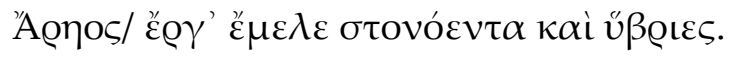

\section{(2) Abwägung bzw. Kombination der Varianten mehrfach überlieferter Stücke}

Eine in der Textkritik der Theognideen häufig zum Erfolg führende Verfahrensweise ist die kombinierende Abwägung mehrerer Überlieferungsvarianten, ein Verfahren, welches sich insbesondere dann anbietet, wenn Textsegemente entweder innerhalb der Theognideen mehrfach überliefert sind oder wenn eine Parallelüberlieferung außerhalb der Theognideen existiert:

\footnotetext{
${ }^{2}$ Nach van Herwerden (1870: 14) "subabsurde", nach Hudson-Williams "mock-heroic on the analogy of

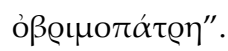




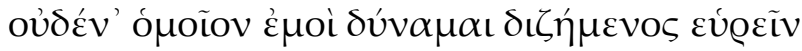

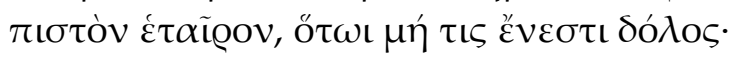

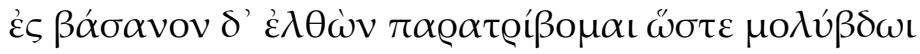

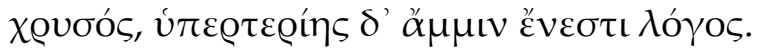

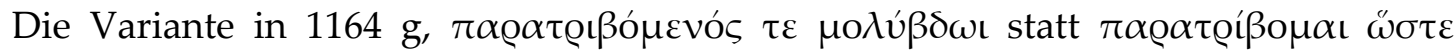
$\mu \mathrm{o}\langle\hat{\beta} \beta \delta \omega \mathrm{l}$ (verbunden mit einem Fehlen des $\delta$ 'im folgenden Vers), erweist sich durch die formal anstößige enge Verbindung zwischen aoristischem und praesenti-

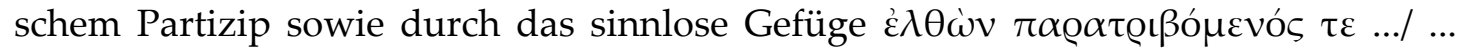

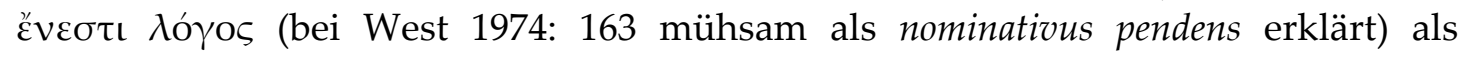

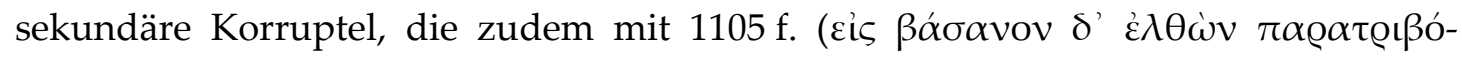

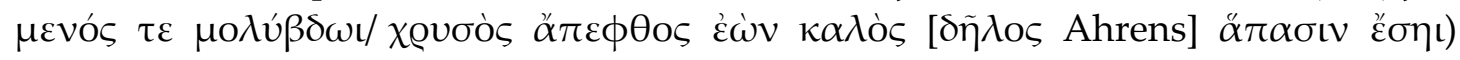

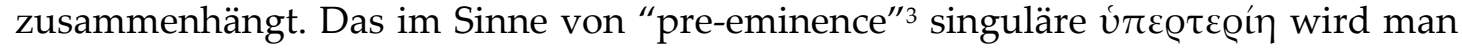
jedoch auch in der Textfassung von $417 \mathrm{f}$. nicht akzeptieren, zumal sich eine einfache

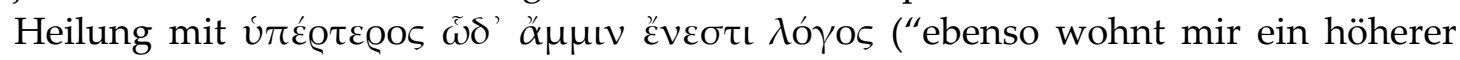

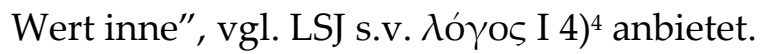

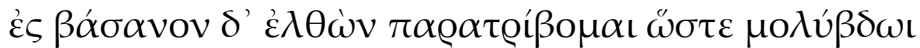

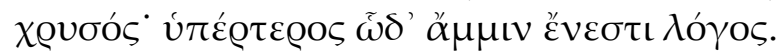

Darüber hinaus könnte man erwägen, mit einem weiteren leichten Eingriff das dem Sprecher günstige Ergebnis des Vergleichs zu einem vom Gleichnis zur Wirklichkeitsebene zurückleitenden Hauptsatz auszugestalten:

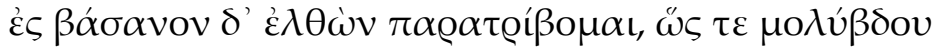

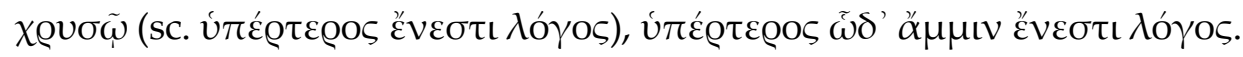
„... und wie dem Golde im Vergleich zum Blei, so wohnt auch mir höherer Wert inne“.

$441-446=1162 \mathrm{a}-\mathrm{f}$

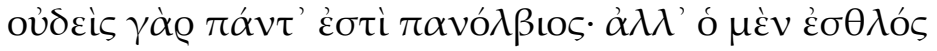

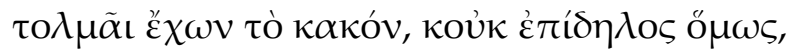

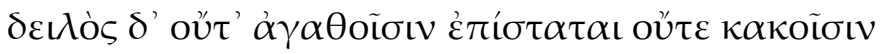

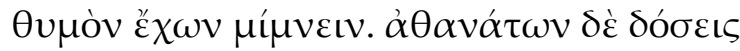

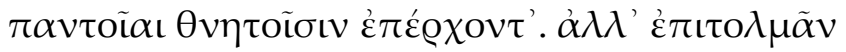

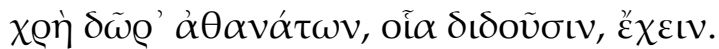

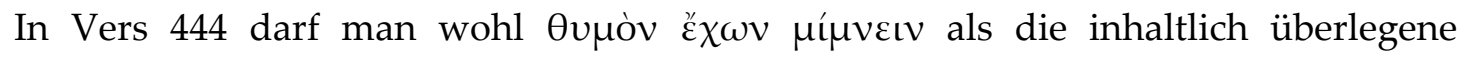

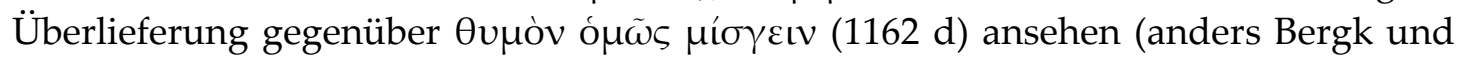
Hudson-Williams): Wenn sich ó $\mu \tilde{\omega} \varsigma$ auf die beiden denkbaren Fälle $\alpha \gamma \gamma \alpha \theta$ oĩ $\sigma \iota v$ bzw.

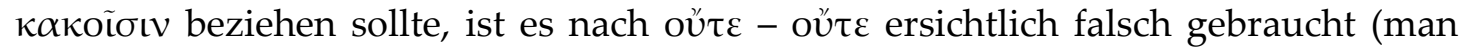
erwartet ein ,sowohl - als auch“), und die ganze Variante ist verdächtig, auf einem nachträglichen Verbesserungsversuch eines Redaktors zu beruhen. Wenn man

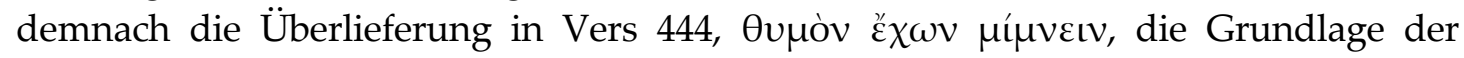

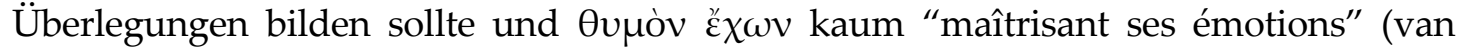

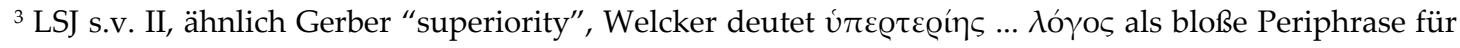

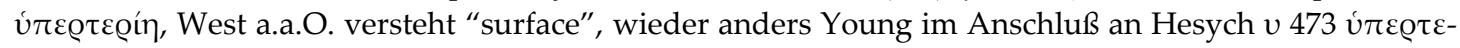

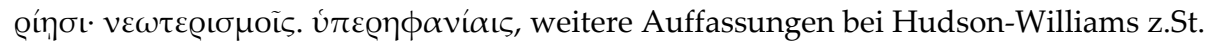

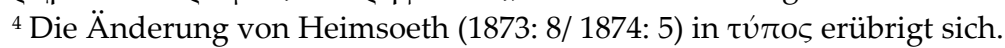


Groningen ohne passende Parallelen, ähnlich Gerber "to control his emotions") bedeuten kann und das alleinstehende $\theta v \mu$ òv $\varepsilon^{\prime} \chi \omega v$ auch nicht von 1178 a gestützt

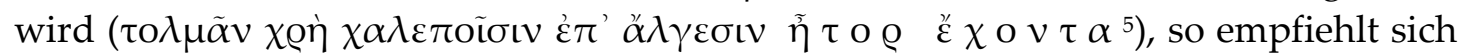
die Herstellung eines Ausdrucks der Art "einen (im Guten wie im Schlechten)

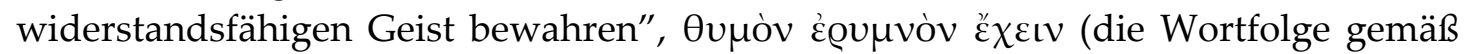

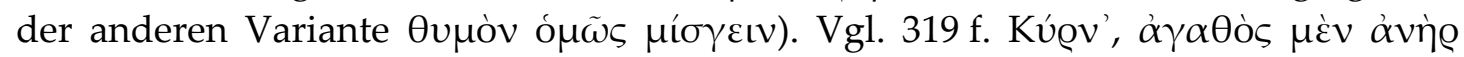

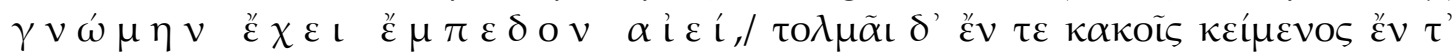

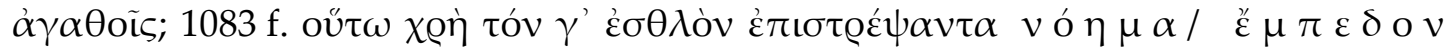

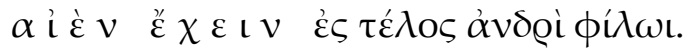

$523-526$

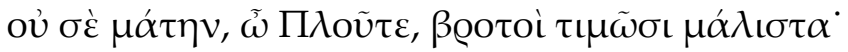

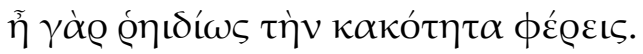

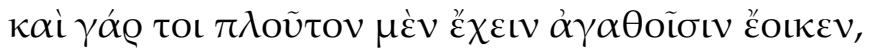

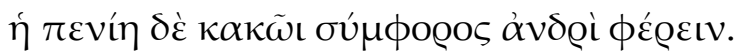

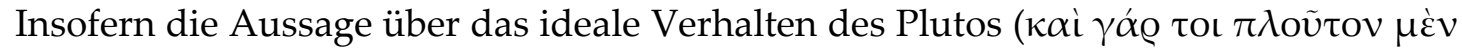

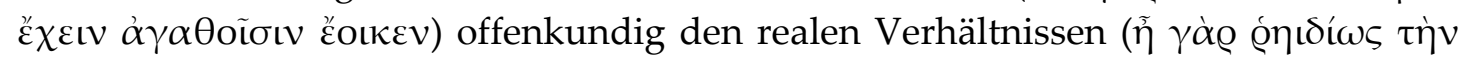

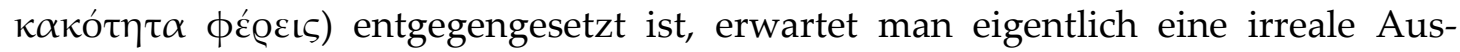

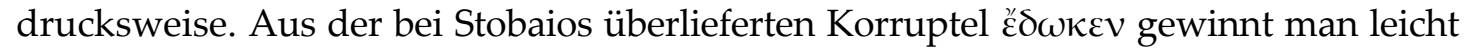
als Urfassung das Imperfekt $\dot{\varepsilon} \omega \kappa \varepsilon \varepsilon$.

$963 \mathrm{f}$.

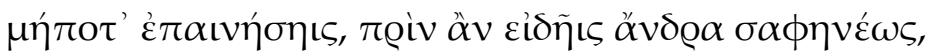

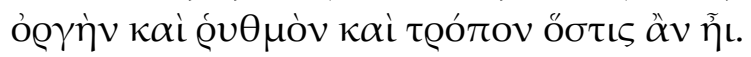

An der oben wiedergegebenen Variante der direkten Überlieferung befremdet die

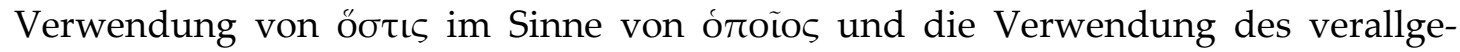
meinernden Relativsatzes anstelle eines indirekten Fragesatzes. Stobaios überliefert

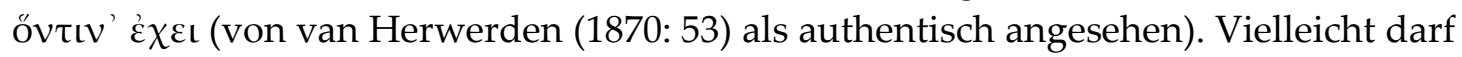

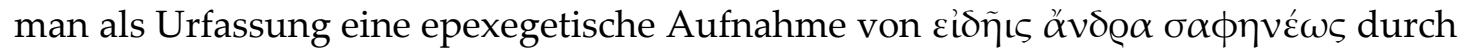

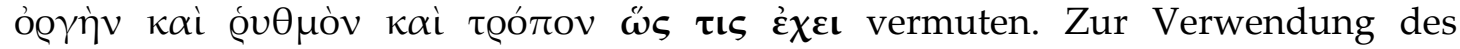
einfachen Relativpronomens nach Verben des Wissens vgl. Kuehner/ Gerth II 438 f.;

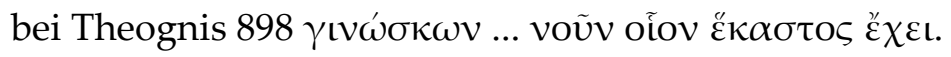

$1095 \mathrm{f}$.

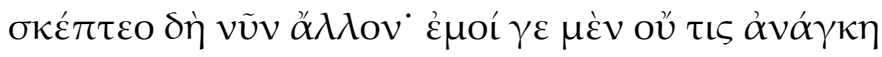

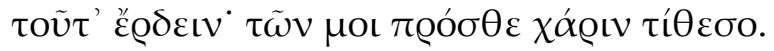

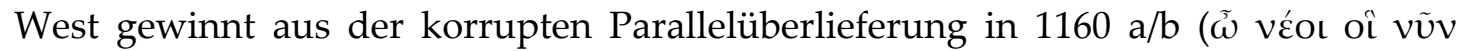

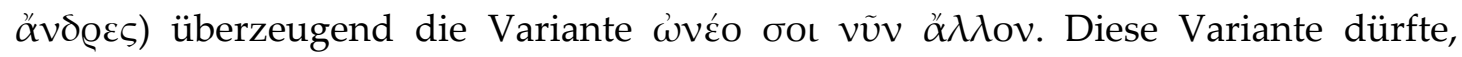

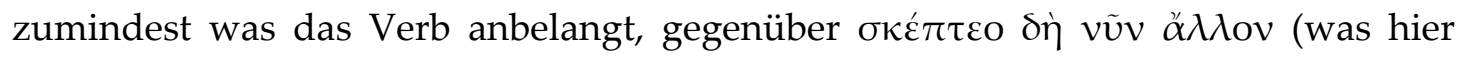

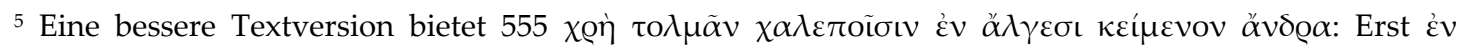

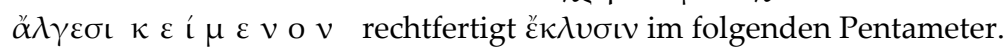


heißen müßte "suche nach einem anderen", nicht "betrachte einen anderen") entschieden den Vorzug verdienen, zumal sie sich mit der ebenfalls an der späteren Stelle überlieferten Variante $\tau \alpha \tilde{v} \tau^{\prime} \varepsilon^{\prime} \rho \delta \varepsilon เ v$ (in der üblichen falschen Orthographie $\tau \alpha \tilde{v} \theta^{\prime}$ $\varepsilon ๊ \rho \delta \varepsilon v)$ zu einem vorzüglichen Sinn zusammenschließt, wenn man nur $\tau \tilde{\omega} v$ als Relativpronomen und $\tau i ́ \theta \varepsilon \sigma o$ als augmentloses Imperfekt auffaßt ${ }^{6}$ :

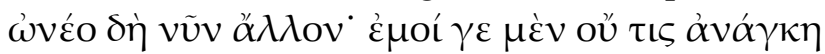

$\tau \alpha \tilde{v} \tau$ '

"Kauf dir (durch deine Gefälligkeiten) einen anderen: Ich jedenfalls habe es nicht nötig, das zu tun, wofür du dir (schon) im Vorhinein bei mir (durch deine Zuwendungen) Dank hinterlegt hast".

$1107 \mathrm{f} .=1318 \mathrm{ab}$

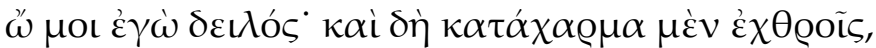

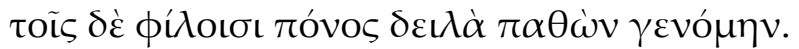

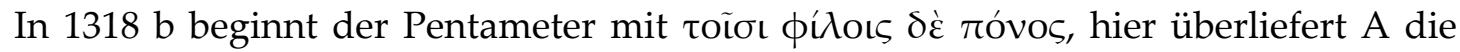

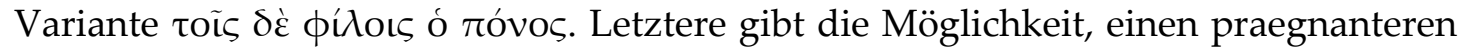

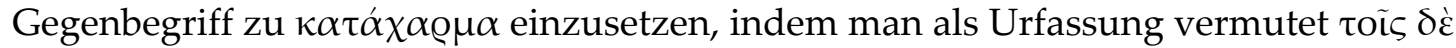

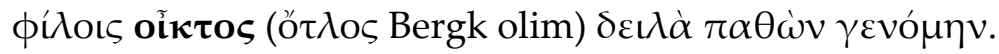

1159 f. (erweitert durch Stob. IV 31, 26)

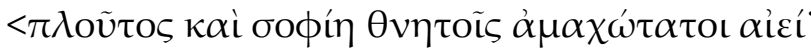

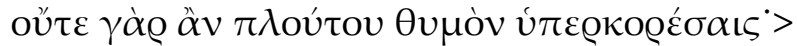

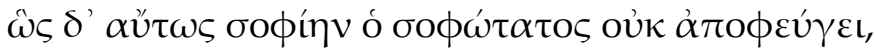

$\alpha \lambda \lambda$ '

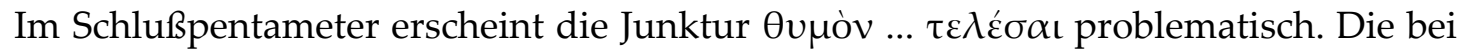
Hudson-Williams und van Groningen danebengestellten homerischen Wendungen

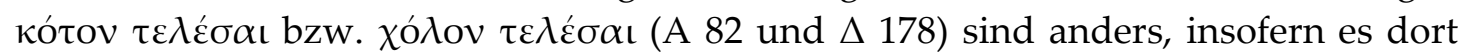
nicht um die Sättigung einer psychischen Instanz, sondern die Befriedigung eines

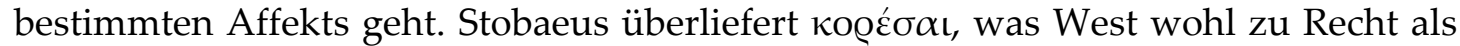
Influenzfehler aus dem ersten Pentameter ansieht. Vielleicht ist auch $\theta v \mu$ òv als Influenzfehler zu bewerten (der dann bereits in der gemeinsamen Vorlage von Stobaeus und dem Theognideen-Excerptor gestanden haben müßte). Den Grundgedanken des Epigramms (für Reichtum und Weisheit gibt es kein Vollmaß) käme

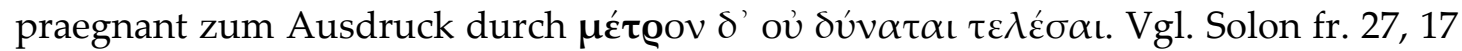

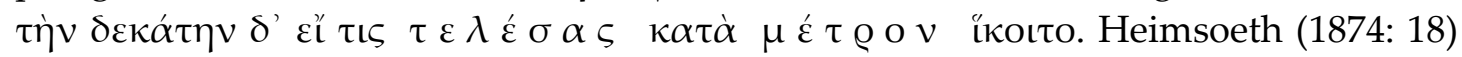

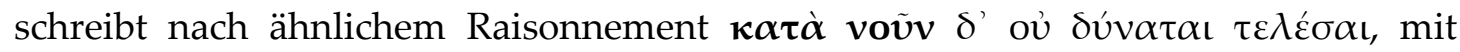

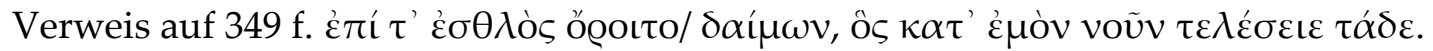

$1161 \mathrm{f}$.

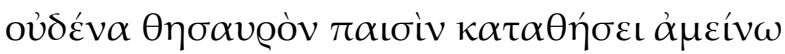

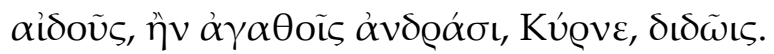

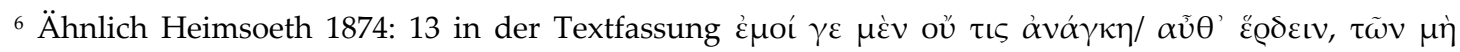

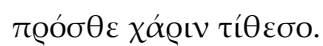


Im zweiten Teil des Distichons muß man in der Tat der Stobaios-Überlieferung

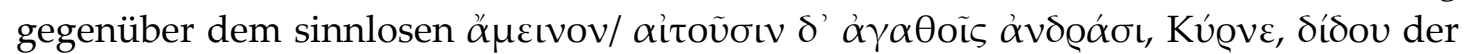
Theognis-Handschriften folgen. Allerdings kann man, abweichend von Wests oben wiedergegebener Textfassung, auf einen Relativsatz zu aiঠoṽ $\varsigma$ kaum verzichten, der

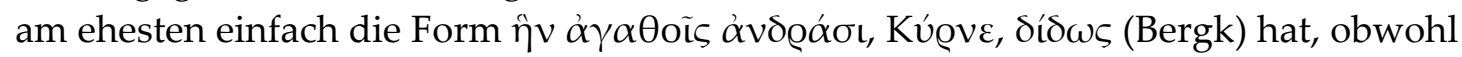
ein generalisierender Konjunktiv $\delta \iota \tilde{\omega} \iota \varsigma$ natürlich nicht auszuschließen ist.

Daß es sich um einen indikativischen Relativsatz handeln sollte, zeigt auch

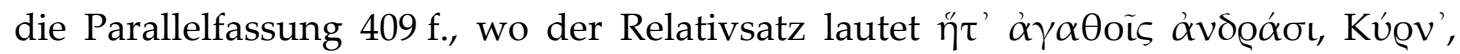
$\varepsilon \check{\tau} \pi \tau \alpha$. Eine inhaltliche Abwägung dieser beiden Varianten soll erst unten im Zusammenhang mit dem Hexameter erfolgen.

Wenn die direkte Überlieferung in dem vorliegenden Distichon ohnehin gestört ist, spricht nichts dagegen, auch im ersten Teil Stobaios zu folgen: $\pi \alpha$ เoív ist

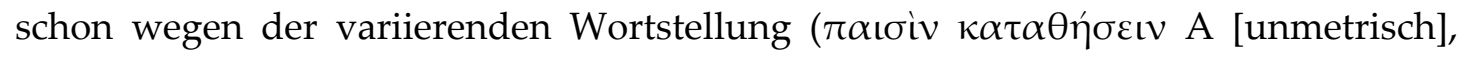

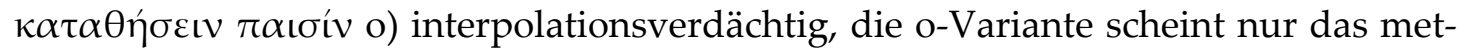
rische Problem zu kaschieren. Ein Alternativversuch, die metrische Schwierigkeit zu beheben, ist die in 409 überlieferte mediale Variante $\pi \alpha \iota \sigma i v \kappa \alpha \tau \alpha \theta \eta ́ \sigma \varepsilon \iota(-\eta \iota)$, die die Metrik mit einer genos-konformen Hiatkürzug elegant in Ordnung bringt, aber andererseits das Medium sinnwidrig mit dem Dativus commodi $\pi \alpha \iota \sigma i ́ v$ verbindet.

Inhaltlich erscheint ein Rekurs auf "Kinder" als Nutznießer des "thesaurisierten" moralischen Guts unnötig umständlich: Der Nutznießer wird der Angesprochene selbst sein, was in der medialen Ausdrucksweise $\kappa \alpha \tau \alpha \theta \eta ́ \sigma \varepsilon \alpha \iota ~ \varepsilon ้ v \delta o v$

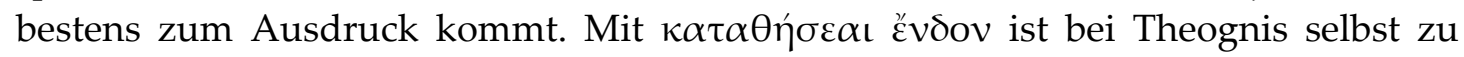

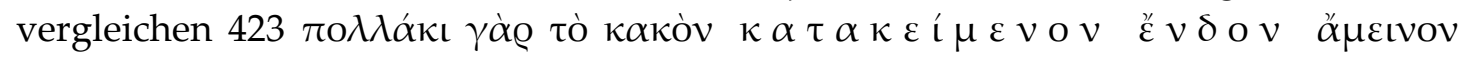
(wo sich die Junktur etwas anders auf eine Geheimhaltung bezieht), vor allem aber

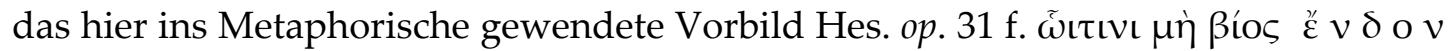

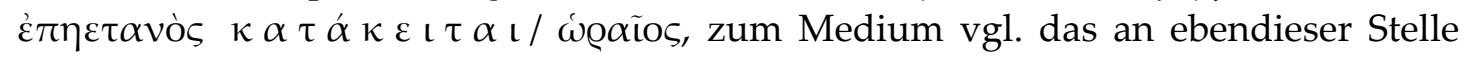

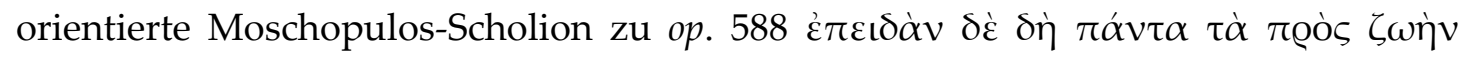

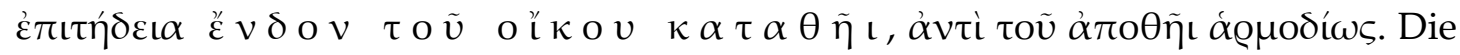
interpolierte Variante der direkten Überlieferung mit $\pi \alpha$ เóv orientiert sich dagegen

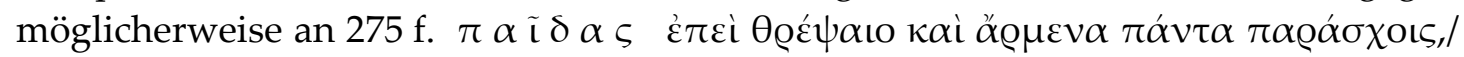

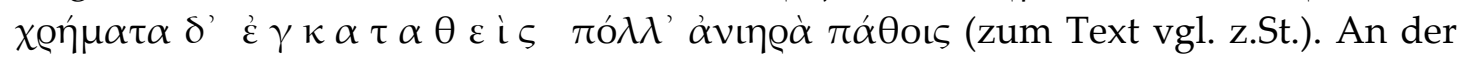
von Hudson-Williams (zu 409 f.) verglichenen Parallelstelle Plat. leg. V 729 b ist charakteristischerweise nicht von "Hinterlegen", sondern von "Hinterlassen" die

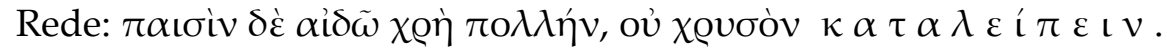

Wenn es demnach in der Urform des Distichons um die $\alpha i \delta \omega \varsigma s$ als ein im eigenen Interesse thesaurisiertes moralisches Gut geht, kommt wenig darauf an,

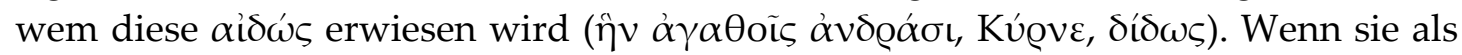
"im Inneren des Hauses eingelagerter Schatz" angesehen wird, so scheint sie vor allem als Indiz der moralischen Überlegenheit des Besitzenden betrachtet zu werden.

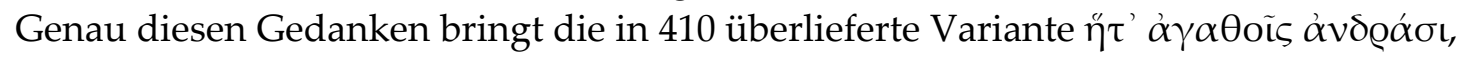

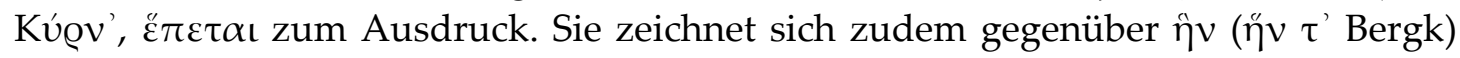

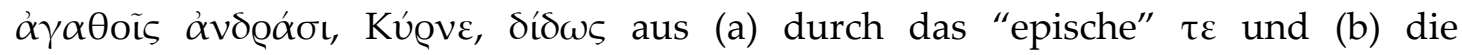
idiomatische Verwendung von ع̊r $\varepsilon \sigma \theta \alpha \mathrm{l}$ (vgl. LfgrE I $4 \mathrm{~b}$ und bes. Theog. 635

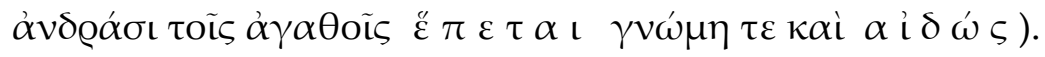

Als Urfassung ergäbe sich also: 


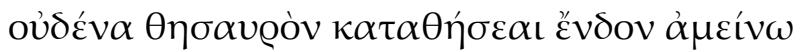

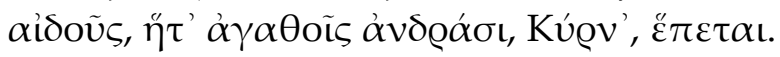

Mit solchem „Kombinationsverfahren" ist gelegentlich auch die Frage nach Versinterpolationen verbunden.

$53-62$

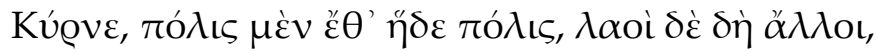

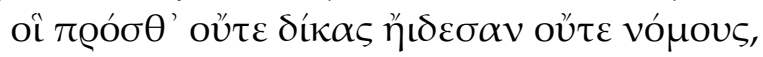

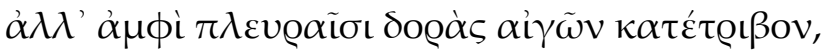

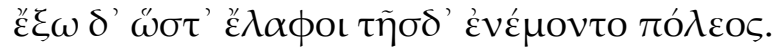

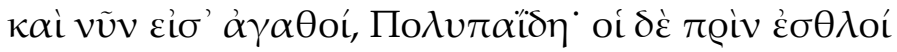

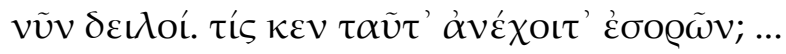

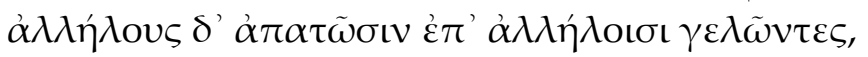

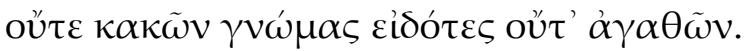

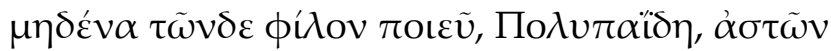

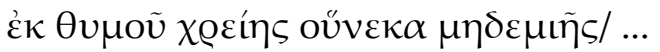

West setzt im Anschluß an Schneidewin eine Lücke nach Vers 58 an, in der ein Exzerptor das Distichon ausgelassen habe, welches in der Parallelüberlieferung 1109-1114 erhalten ist:

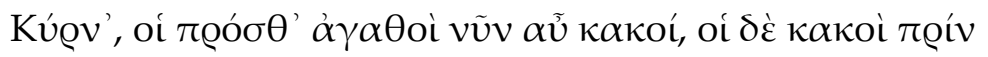

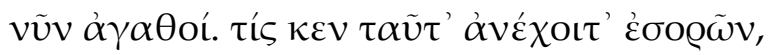

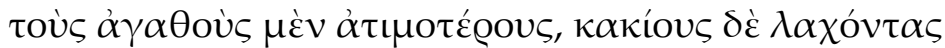

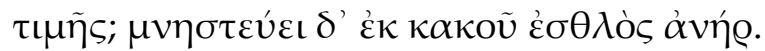

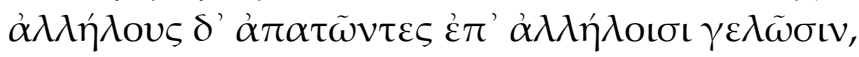

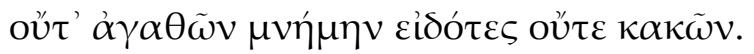

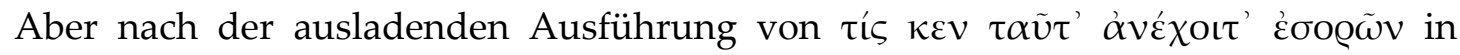
einem vollständigen Distichon fällt es schwer, die einfache 3. Person Plural in 59 bzw. 1113 wieder auf die zuvor genannten Emporkömmlinge zu beziehen. Das Distichon $1111 \mathrm{f}$. läßt sich sehr gut als eine Interpolation verstehen mit dem Zweck,

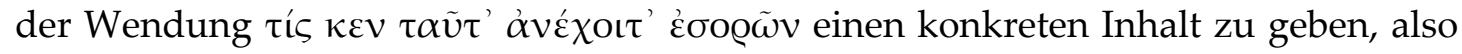
das Demonstrativum zu explizieren (wobei die Heiratsverhältnisse wohl einfach der Komplettierung des Distichons dienen, das sich nicht ganz mit dem Gedanken der Statusvertauschung füllen ließ).

Auch sonst tragen die Verse 1109-1114 alle Merkmale sekundärer Bearbei-

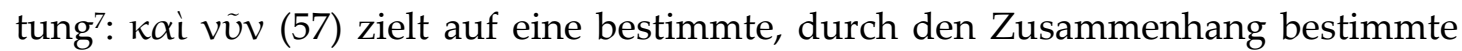
politische Situation (nach dem "Austausch" der Bürger, 53-56), während das einleitende $\operatorname{Kú} \varrho v(\varepsilon)$ in 1109 eine denkbar allgemeine Betrachtung praepariert. In dem ursprünglichen Gedicht geht es primär um die emporgekommenen Neubürger, die ehemaligen $\dot{\varepsilon} \sigma \theta \lambda$ oí kommen nur in der Form eines parenthetischen "Stoßseufzers" aus oligarchischer Perspektive (zu dieser Interpunktion s.u.) ins Blickfeld, während es dem sekundären Bearbeiter in $1109 \mathrm{f}$. lediglich um eine begrifflich geglättete Gegenüberstellung von "Gut" und "Böse" geht.

\footnotetext{
7 Vgl. schon Reitzenstein 1893: 63 f. und Friedländer 1913: 579 f. Anm. 3; anders Steffen 1967/ 8, der in 1109-1114 eine sekundäre Bearbeitung des Theognis selbst sieht.
} 


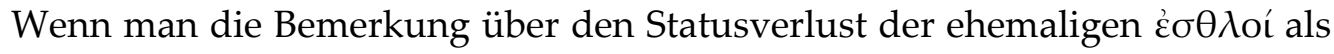
Parenthese faßt, schließen sich die Verse 59 f. ohne Schwierigkeit an $57 \mathrm{f}$. an:

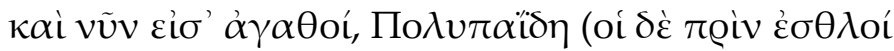

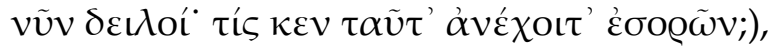

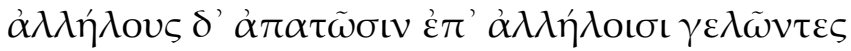

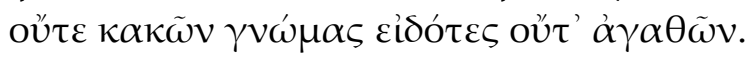

Mit $\dot{\alpha} \lambda \lambda \eta \dot{\lambda o v \varsigma} \delta$ ' $\alpha \pi \alpha \tau \tilde{\omega} \sigma \iota v$ wird eine schneidende Widerlegung des Selbstverständnisses der selbsterkorenen $\dot{\alpha} \gamma \alpha \theta$ oí eingeleitet: Sie beanspruchen, $\dot{\alpha} \gamma \alpha \theta$ oí zu sein, führen sich aber gegenseitig in geradezu läppischer Weise hinters Licht ( $\dot{\alpha} \pi \alpha \tau \tilde{\alpha} v$ muß Hauptverb sein, 59 ursprünglich gegenüber 1113) und bekunden damit das

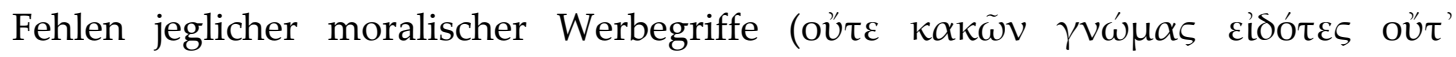

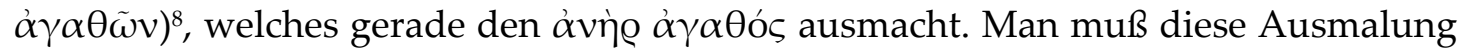

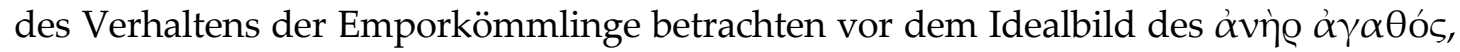
der sich infolge seiner von vorneherein gefestigten $\gamma \nu \omega \mu \eta \eta$ eben nicht hinters Licht führen läßt (1037 f., zum Text vgl. z.St.):

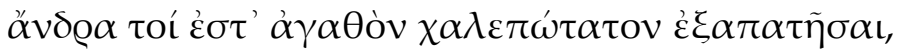

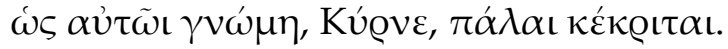

In Vers 61 beginnt eine als separates Gedicht zu betrachtende Ermahnung an Kyrnos, sich keinen der "Städter" zum Freund zu machen. Darin bekundigt sich das typisch oligarchische Mißtrauen gegen Mitglieder des Demos. Die emporgekommenen Neubürger, denen in 53-60 eben nicht der Titel "Bürger" gegeben wird, sondern die unter den wohl bewußt abqualifizierend gebrauchten Begriff $\lambda \alpha$ oí (53) zu subsumieren sind, würde Theognis kaum unvermittelt als $\tau \tilde{\omega} v \delta \varepsilon \ldots . \dot{\alpha} \sigma \tau \tilde{\omega} v$ (61) bezeichnet haben.

Gelegentlich läßt sich mit diesem Verfahren auch ein innerhalb der Theognideen sekundär überlieferter älterer Autor rekonstruieren.

719-728 = Solon fr. 24 W. $=18$ G./P.

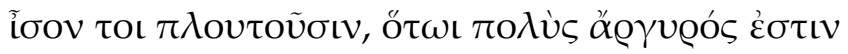

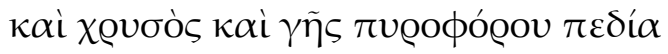

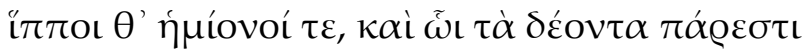

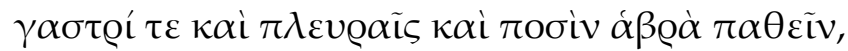

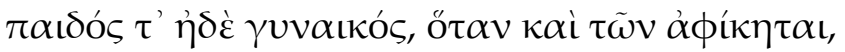

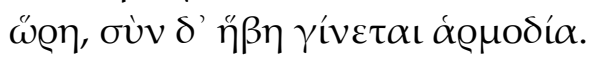

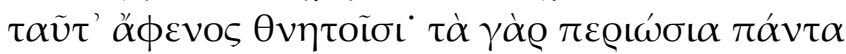

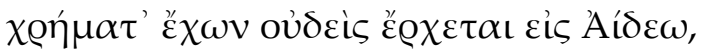

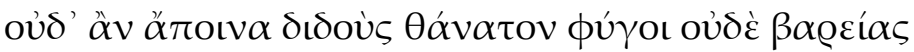

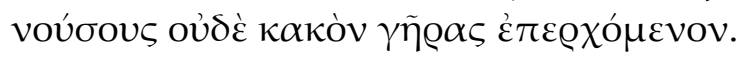

Die folgenden Bemerkungen dienen dem Zweck, eine sinnvolle (wahrscheinlich solonische) Urform aus den bei Theognis, Plutarch und Stobaios überlieferten Varianten zu rekonstruieren.

\footnotetext{
${ }^{8}$ Hudson-Williams' Deutung " $\gamma \nu \omega ́ \mu \eta=\gamma \nu \omega ́ \varrho \iota \sigma \mu \alpha$, distinctive mark of good and bad men" scheitert schon am Plural $\gamma v \omega \dot{\mu} \alpha \varsigma$.
} 


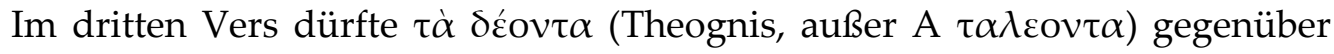

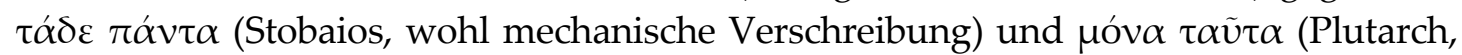
vgl. West 1974: 158) das Ursprüngliche treffen. Den zuvor aufgezählten materiellen

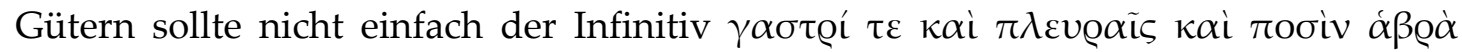
$\pi \alpha \theta \varepsilon \tilde{\varepsilon} v$ entgegentreten, sondern vielmehr die für dieses $\alpha \beta \mathrm{O} \alpha \dot{\alpha} \pi \theta \varepsilon \tilde{\varepsilon} v$ erforderlichen

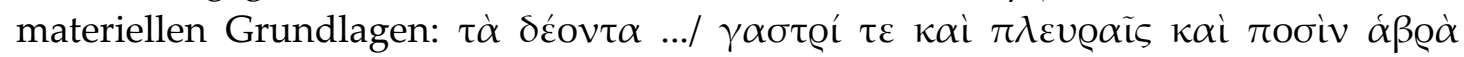
$\pi \alpha \theta \varepsilon \tilde{\imath} v$. Wichtig ist eine Bemerkung Kaibels (Crönert 1925: 314, vgl. auch

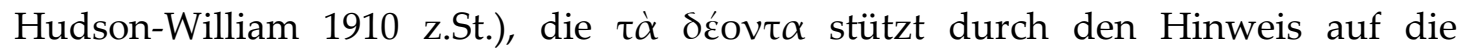
horazische Imitation in epist. I 12, 4-6: Pauper enim non est cui re ru $m$ sup pe tit us us./ Si ventri bene, si lateri est pedibusque tuis, nil/ Divitiae poterunt regales addere maius. In der Tradition solcher popularphilosophischer Wunschkataloge steht ferner Mart. II 48, wo auch ein Knabe (5) zu den erwünschten Gütern gehört.

$\mathrm{Zu}$ diesen materiellen Grundlagen gesellt sich im dritten Distichon (bei Horaz

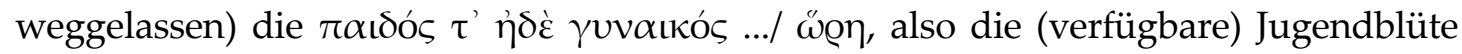
eines männlichen oder weiblichen Sexualpartners. Im dritten Hexameter bieten

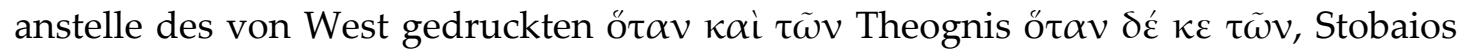

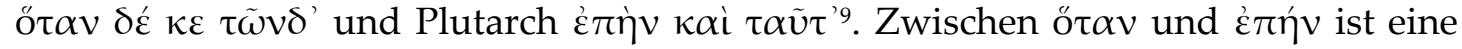
Entscheidung kaum möglich. Was die nachfolgenden Worte anbelangt, so müßte ein

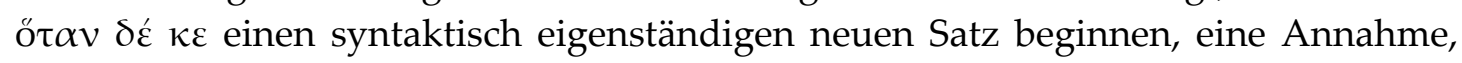
die zu erheblichen Schwierigkeiten führt (vgl. West 1974: 158). Sowohl $\delta \varepsilon ́ \kappa \varepsilon$ als auch $\kappa \alpha$ í lassen sich ohne weiteres als Lückenfüller eines verstümmelten Texts begreifen. $\tau \alpha \tilde{v} \tau$ ' könnte ein Versuch sein, anstatt $\tau \tilde{\omega} v$ notdürftig ein (ausgefallenes) Subjekt zu

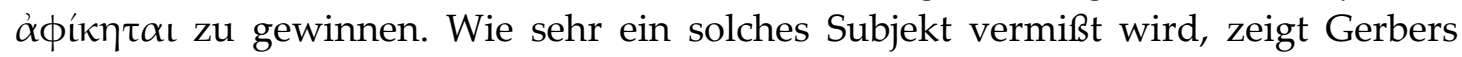

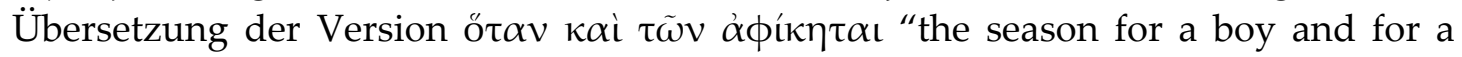

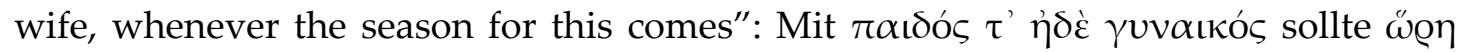
doch eher "Jugendblüte" bedeuten (LSJ s.v. B II 1), während es als zu supplierendes Subjekt in dem Nebensatz ö $\alpha \nu \kappa \alpha i ̀ \tilde{\omega} \nu \dot{\alpha} \phi i ́ \kappa \eta \tau \alpha \iota$ "the fitting time or season for a thing" (LSJ s.v. B I 1) heißen müßte.

Man vermutet den Gedanken, daß die Blüte eines jugendlicher Knabe oder einer jungen Frau im Falle des Aufkommens eines Verlangens danach vorhanden ist. Als Urfassung sei vorgeschlagen:

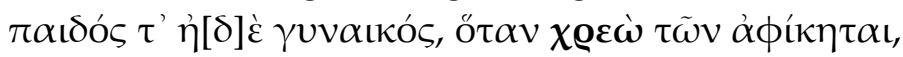
$\omega ̋ \emptyset \ldots$

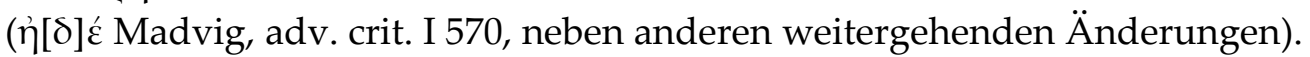

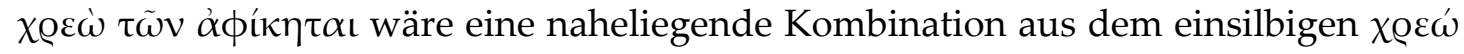

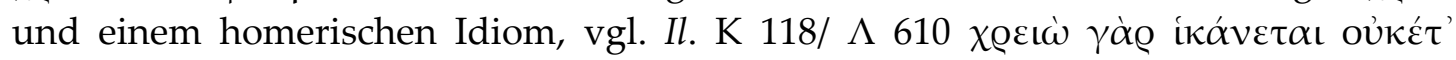

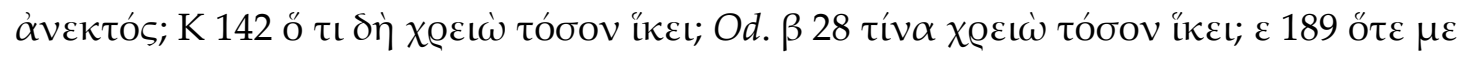

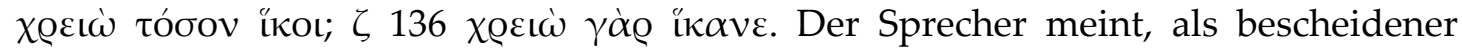
Reichtum genüge es, wenn man genug besitze, um sich ein körperliches Wohlbefinden zu verschaffen, und zudem über einen Knaben oder eine junge Frau verfüge, wenn einen das Verlangen danach überkommt, zugleich aber ( $\sigma u ̛ v ~ \delta ')$ selbst noch in einem hierzu "passenden" Jugendalter sei.

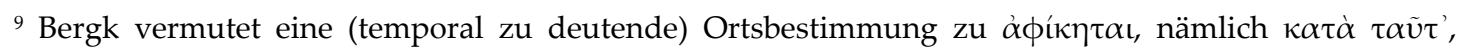

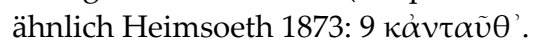




\section{(3) „Flickpartien“}

Eine besondere Rolle spielt in der Editionsgeschichte der Theognideen die Annahme von „Flickpartien“. Häufig liegen plausible Annahmen, was sich hinter solchen „Flickstücken" verbergen könnte, nicht allzu fern.

193-196

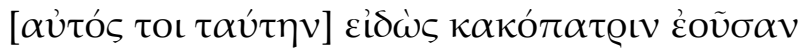

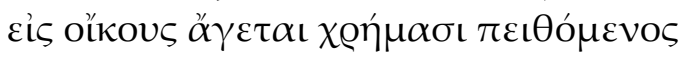

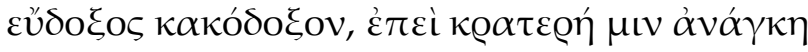

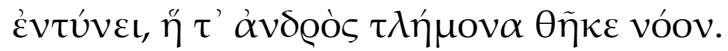

Hudson-Williams' Erklärung von aủtós ("Though the man himself knows, he still marries the woman", ähnlich Gerber 1970: 286) überzeugt in Anbetracht der Spitzenstellung von $\alpha u ̛$ đó kaum. West sondert den ersten Halbvers als "excerptoris supplementum" aus, hinter dem sich dann möglicherweise auf einen konkreten Fall bezügliche Eigennamen verbergen könnten. Alternativ empfähle sich eine konjekturale

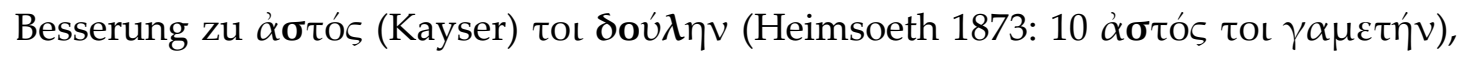
was gut zum ebenfalls antithetischen Auftakt des zweiten Distichons mit $\varepsilon u ̈ \delta o \xi o s$

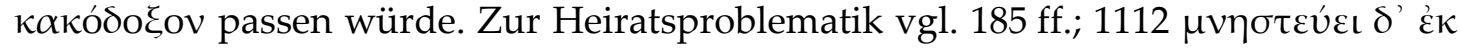

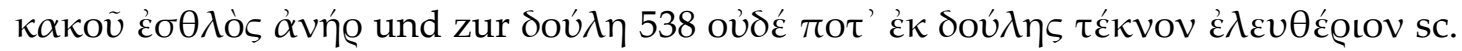

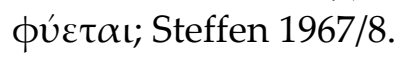

$283-286$

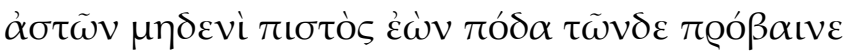

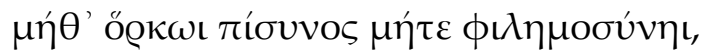

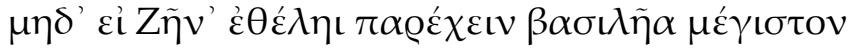

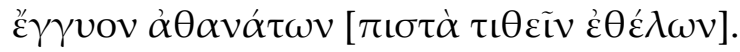

West sondert das zweite Hemistich des Schlußdistichons als "excerptoris supplementum" aus. Die Wiederaufnahme des verbum finitum $\dot{\varepsilon} \theta \dot{\varepsilon} \lambda \eta$ in der Form des Partizips $\dot{\varepsilon} \theta \varepsilon \dot{\varepsilon} \lambda \omega v$ ist in der Tat unerträglich, zumal in Verbindung mit dem nichts Neues hinzugebenden Infinitiv $\pi \iota \sigma \tau \dot{\alpha} \tau \iota \theta \varepsilon \tilde{\imath} v$. Hartung wollte demgegenüber mit dem von ihm konjizierten Imperativ $\varepsilon^{\theta} \theta \varepsilon \lambda \varepsilon$ im zweiten Distichon ein eigenständiges Kondizionalgefüge herstellen (“... wolle es nicht für sicher einschätzen”); in diesem Falle erwartet man übrigens "für sicher einschätzen" mit dem Medium $\pi \iota \sigma \tau \dot{\alpha}$ $\tau i ̂ \varepsilon \sigma \theta \alpha \iota$ ausgedrückt zu finden.

Passend und erwünscht wäre hingegen eine praegnante partizipiale Zusammenfassung der Argumentationsweise des Schwörenden, der mit solchen eidlichen Verweisen auf den höchsten Götterkönig “als Bürgschaft die Sache der Götter vorlegt": $\pi \iota \sigma \tau \dot{\alpha} \tau \iota \theta \varepsilon \grave{\iota} \varsigma \dot{\alpha} \theta \varepsilon \tilde{\omega} v$. Zum Ausdruck in diesem Zusammenhang vgl. [Era-

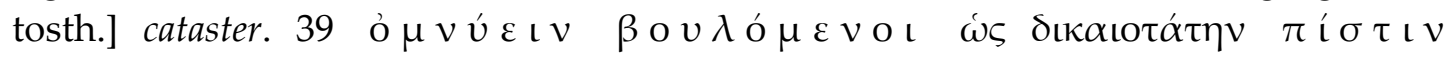
$\tau \iota \theta \varepsilon \dot{\varepsilon} \nu \varepsilon \varsigma$. Dieser Vorschlag wurde in gewisser Weise bereits antizipiert durch

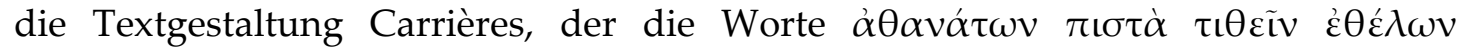




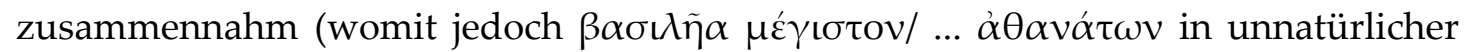
Weise getrennt würde und das Problem $\dot{\varepsilon} \theta \dot{\varepsilon} \lambda \eta \iota . . . /$... $\dot{\varepsilon} \theta \dot{\varepsilon} \lambda \omega v$ unangetastet bliebe).

$287 \mathrm{f}$.

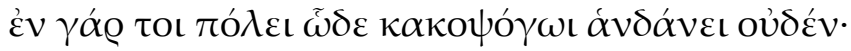

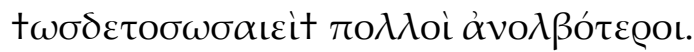

Der Defekt im Pentameter läßt sich verhältnismäßig leicht sanieren mit Ahrens'

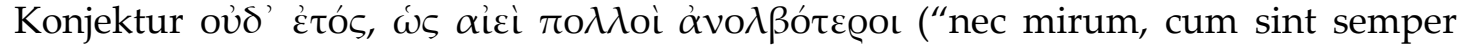
pauci fortunati" in Wests Apparat). Mit dieser Herstellung wird die "demokratische Tadelsucht" aus der Perspektive des oligarchischen Dichters mit typischem Zynis-

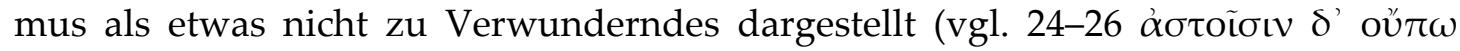

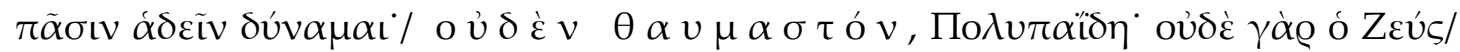

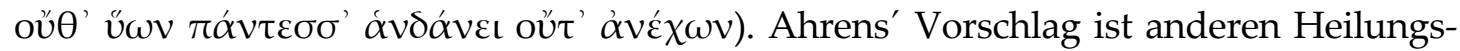
versuchen so weit überlegen, daß man ihn akzeptieren sollte trotz der berechtigten Bedenken von Sitzler (1878: 9), der darauf hinweist, daß der Ausdruck sonst nur in attischer Sprache belegt ist und auch anders verwendet wird, insofern nämlich où

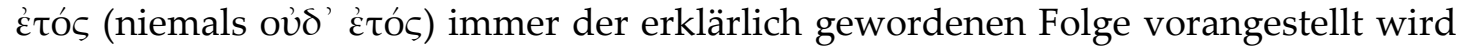

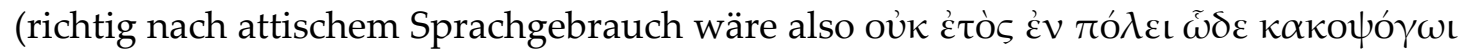

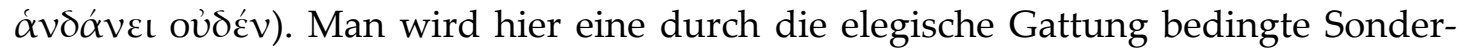
form der Ausdrucksweise annehmen müssen. Eine weitere vereinzelte Abweichung von der attischen Verwendungsweise findet sich in kaiserzeitlicher Epik, Opp. Cyn. I

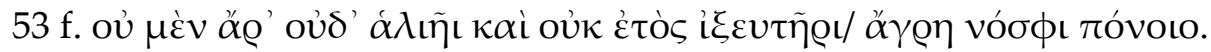

$\mathrm{Ob}$ der Hexameter in der überlieferten Form heil ist, darf man ebenfalls

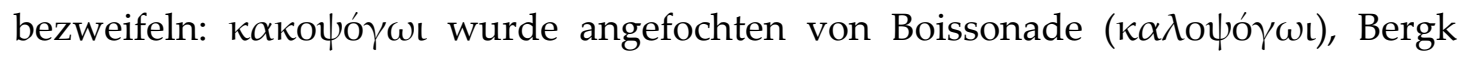

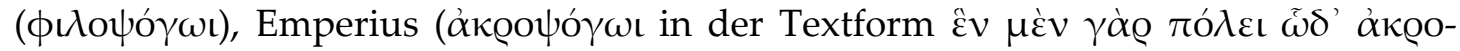

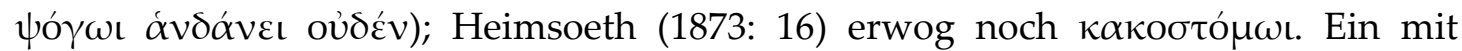
к $\alpha$ Ко- zusammengesetztes Adjektiv der Bedeutung "malignantly blaming" (LSJ, gemäß Hudson-Williams z.St., vgl. auch Sitzler (1878: 8): “maligne vituperans") wäre freilich sehr passend als Kontrast zu einem mit $\varepsilon v$ - zusammengesetzten adjektivi-

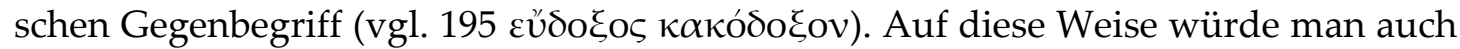

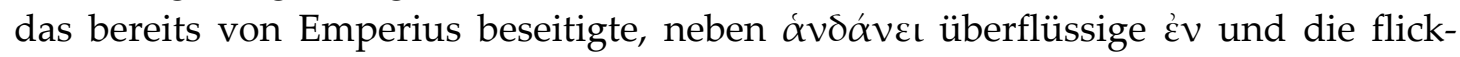

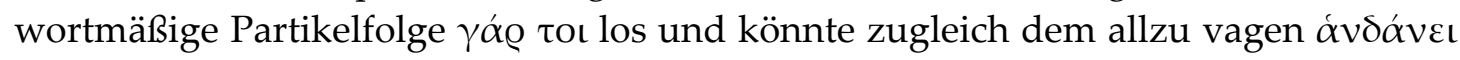
oủঠév konkreten politischen Inhalt geben:

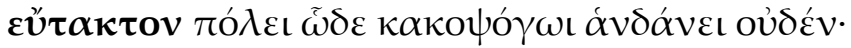

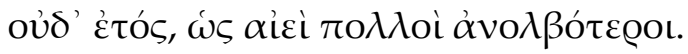

Die aristokratische, anti-demokratische Konnotation des Begriffs عút $\alpha \kappa \tau o \varsigma$ zeigt sich

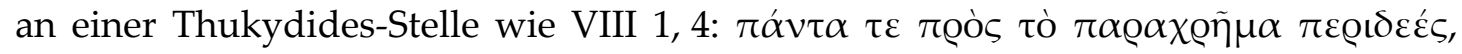

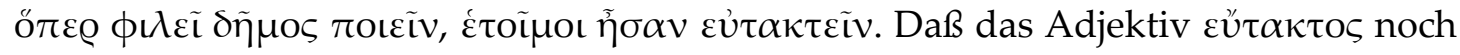
älter ist, zeigt Aisch. Pers. 399.

$625 \mathrm{f}$.

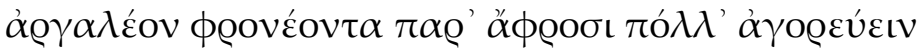

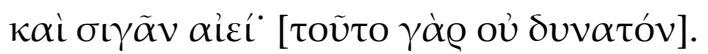


Man fragt sich warum es für den Verständigen in der Nähe von Unverständigen gerade schwierig sein soll, vieles zu sprechen ( $\pi$ ó $\left.\lambda \lambda^{\prime} \alpha \dot{\alpha} \gamma o \varrho \varepsilon v ́ \varepsilon \iota v^{10}\right)$. Prinzipiell müßte sich die Schwierigkeit auch bei nur singulärer Meinungsäußerung ergeben.

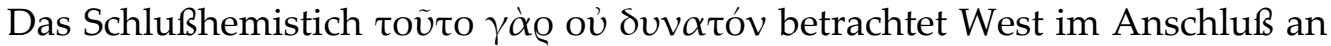
Hudson-Williams als "excerptoris supplementum", Peppmüller (1885: 235) änderte

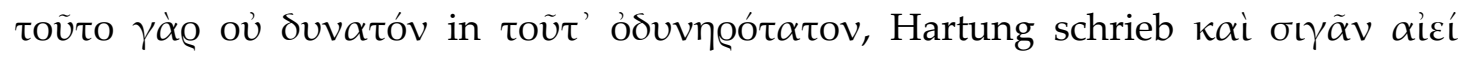

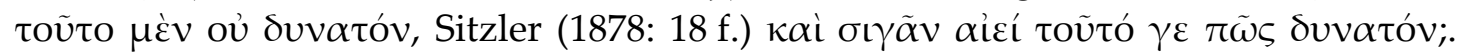

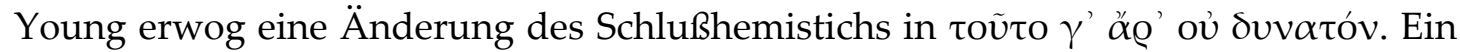
pointierter Schlußsatz im Sinne eines tertium non datur ließe sich mit folgender konjekturaler Textgestaltung gewinnen:

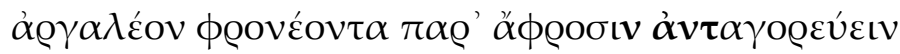

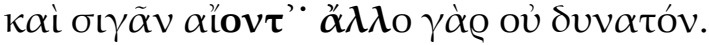

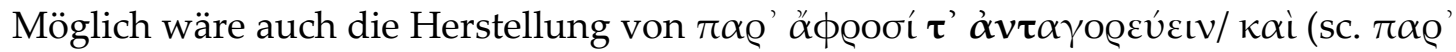

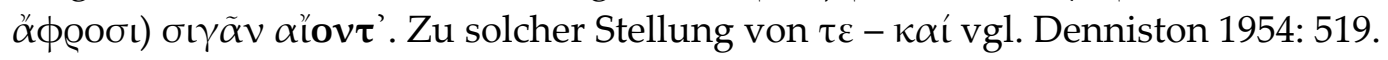

$687 \mathrm{f}$.

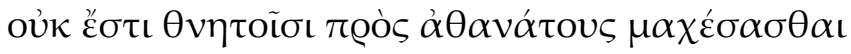

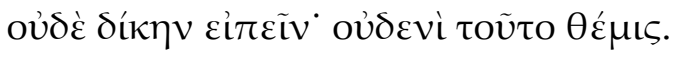

West (1974: 157 f.) sieht in dem Distichon eine gleichnishafte Verdeutlichung der Schwierigkeit einer adaequaten Rechtssprechung; aber könnte man eine solche durch politische Umstände bedingte Schwierigkeit bzw. Unmöglichkeit passend beschrei-

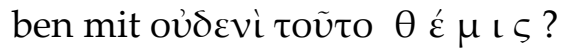

Stilistisch befriedigender als das auch mit dieser Deutung nachklappernde

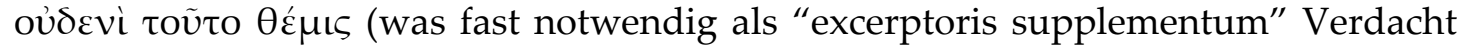
erwecken muß, vgl. West 1974: 158) wäre eine synonymische Korrespondenz des Pentameters zum Hexameter (wobei das Distichon dann wieder als rein gnomische Aussage über die Unterlegenheit von Menschen gegenüber Göttern aufzufassen

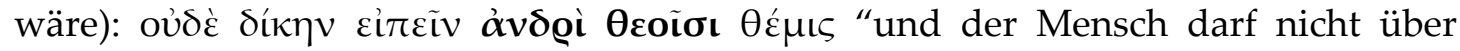
Götter Recht sprechen". Dann wäre die Entstellung des Pentameters durch einen quasi-haplographischen Ausfall von $\theta \varepsilon$ ĩ $\sigma \iota$ vor $\theta \varepsilon \dot{\mu} \mu \varsigma$ und einen anschließenden Ergänzungsversuch bedingt.

$1037 \mathrm{f}$.

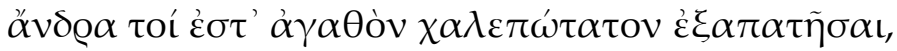

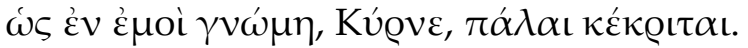

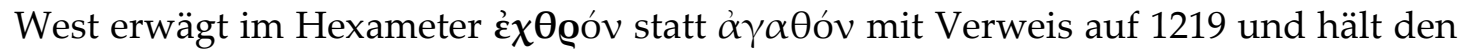
Pentameter für einen "versus ab excerptore suppositus". Eher als die Sinntendenz des Hexameters zu ändern und den Pentameter zu einer gnomologischen Platitüde zu degradieren (das in A überlieferte $\dot{\varepsilon} v \dot{\varepsilon} \mu o \grave{~} \gamma \nu \omega \dot{\mu \eta}$ wird in der übrigen Tradition weiter trivialisiert zu $\dot{\varepsilon} v \dot{\varepsilon} \mu \tilde{\eta} \iota \gamma \nu \omega ́ \mu \eta \iota)$, empfiehlt es sich, aus dem Pentameter eine

\footnotetext{
10 Young verweist auf die hier völlig unpassende Nuance der Geschwätzigkeit, van Herwerden (1870:

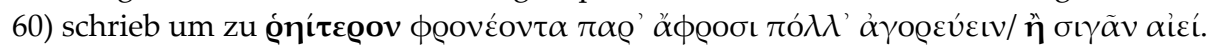


Begründung zu gewinnen, warum der "gute Mann" so schwer hinters Licht zu

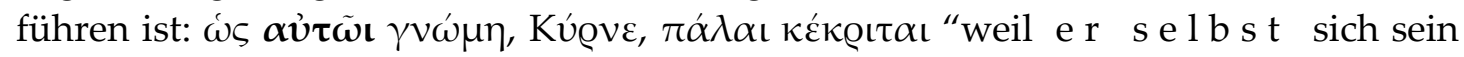
Urteil schon längst gebildet hat". Zum Ausdruck vgl. Phot. Amphiloch. 149 vol. V p.

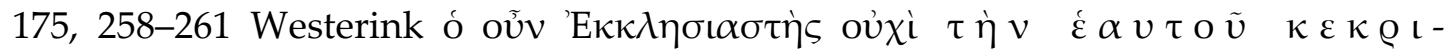

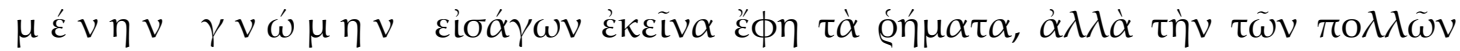

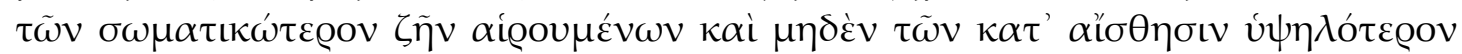

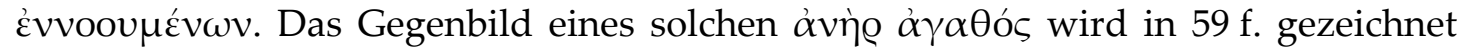
(vgl. z.St.): Es handelt sich um Emporkömmlinge, die sich infolge fehlender Wert-

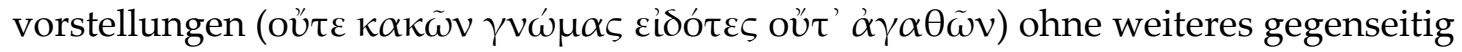
hinters Licht führen.

$1171-1176$

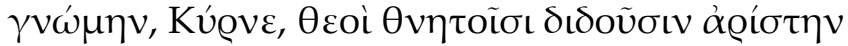

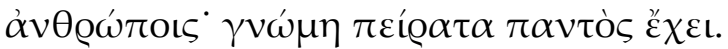

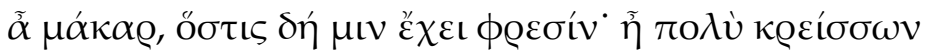

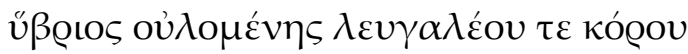

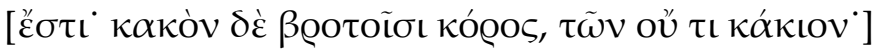

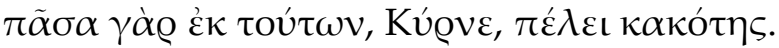

West (1974: 163 f.) tilgt den letzten Hexameter als "versus ab excerptore consutus ut 1176 adnecteret". Vielleicht läßt sich aber sowohl das nachklappernde ع̌ $\sigma \tau t$ als auch die grammatische Schwierigkeit um $\tau \tilde{\omega} v$ beseitigen, wenn man das Schlußdistichon folgendermaßen gestaltet:

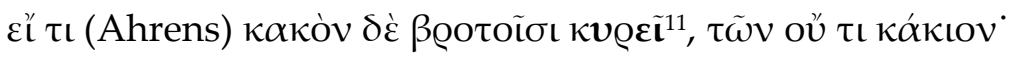

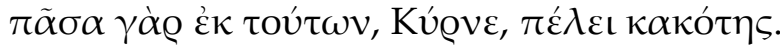

“Wenn Sterblichen nur irgendein Übel begegnet, so gibt es nichts Schlimmeres als die beiden genannten (v̌ß@ı und kó@oৎ). Denn jegliche Schlechtigkeit, Kyrnos, ergibt

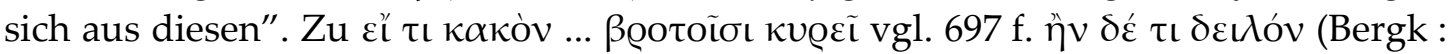

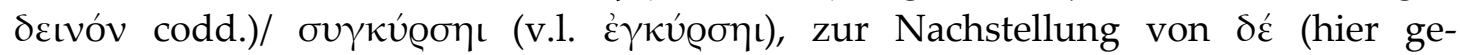

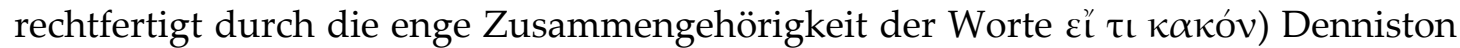
1954: 187-189; zu ähnlichen Nachstellungen von Partikeln auch West (1974: 112). Wests Auffassung (1974: 101 und 164), daß oǔtıs im Sinne von oúdzís in den Theognideen unzulässig sei, erscheint willkürlich, vgl. 787 (adj.) und 1187 (subst.).

\section{(4) unkenntlich gewordene "Fernbeziehungen"}

Mitunter werden innerhalb der kurzen Textsegmente strukturelle „Fernbeziehungen" durch Textkorruptel unkenntlich.

$105-112$

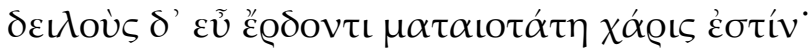

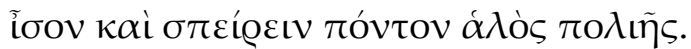

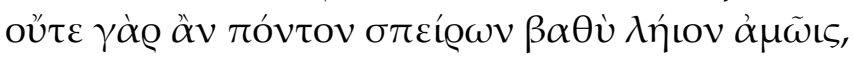

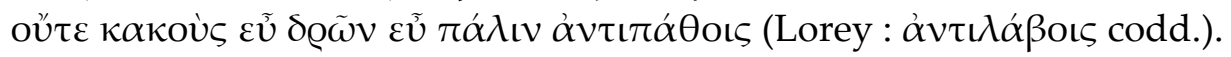

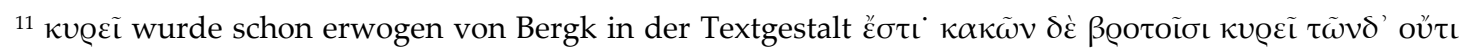
кókı๐.
} 


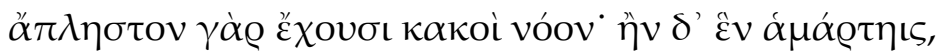

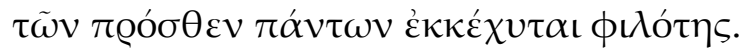

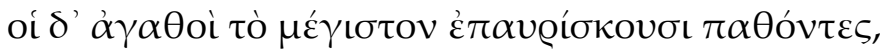

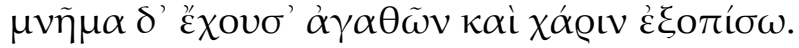

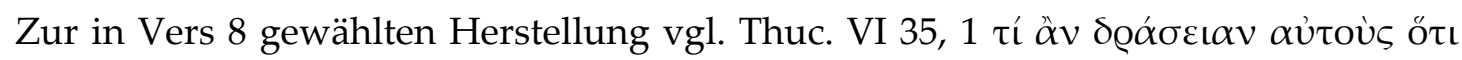

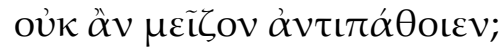

In Vers 111 müßte nach Bergk entweder "boni quovis beneficio contenti sunt" oder "boni vel mala, quae ipsis illata sunt, non curant" gemeint sein. Im Sinne der

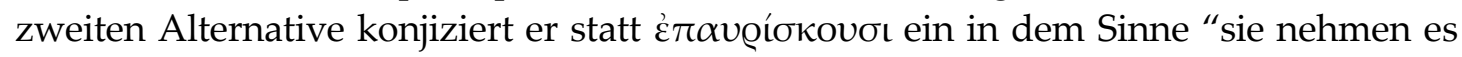

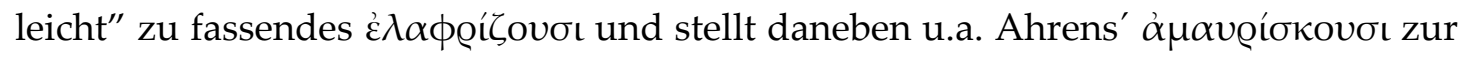
Wahl, das noch in den Apparaten von Young und West mitgeführt wird. Aber im Mittelpunkt des Gedichts steht nicht, wie nachtragend verschiedene Menschengruppen gegenüber zugefügtem Unrecht sind, sondern ob sie Dank für Wohltaten

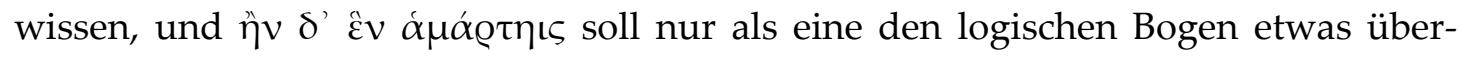
spannende Fallsetzung die Schlechtigkeit der какои́ besonders ins Licht setzen. Die

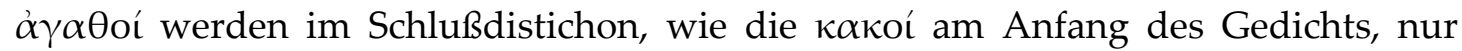

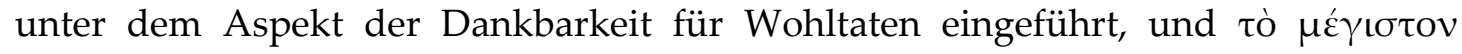

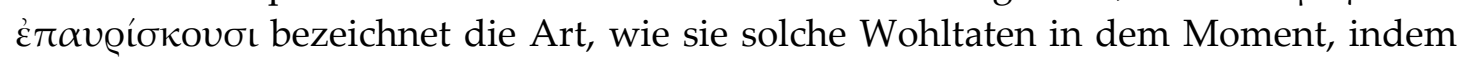
sie sie erfahren haben, goutieren (im Gegensatz zu der später empfundenen Dank-

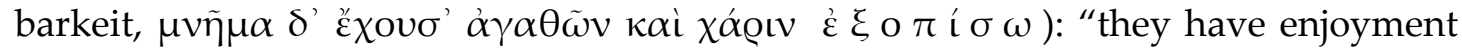
in the highest degree" (LSJ s.v. I 1, anders West (1974: 151): "they remain aware of what they have mainly had from you"). Nur muß der Gedanke, daß es sich bei dem "Erlittenen" um Wohltaten handelt, deutlich zum Ausdruck kommen"12; erst dann

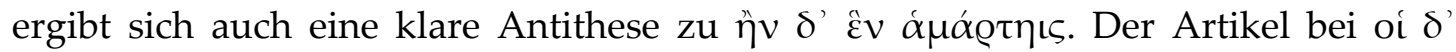

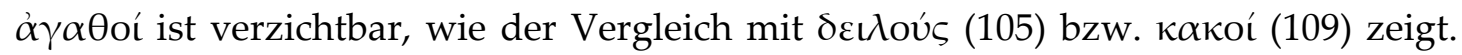
Wenn man schreibt

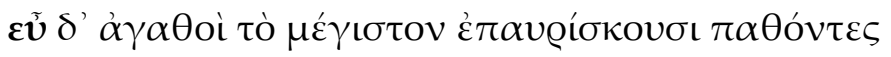

"die Guten empfinden den größten Genuß an erfahrenen Wohltaten", so wird

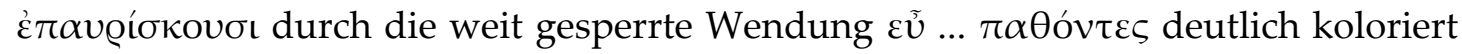
im Sinne der bei LSJ gegebenen Bedeutung. Die starke Sperrung (ähnlich, aber

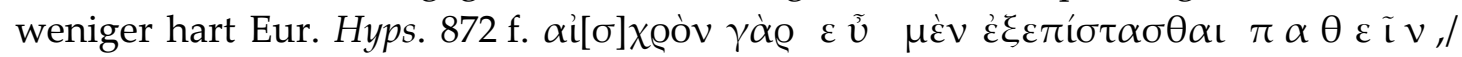

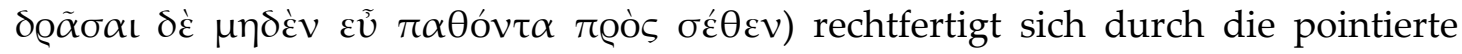

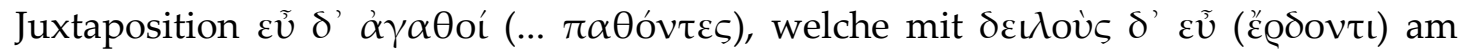
Gedichtanfang anläßlich der Exposition des Verhaltens der antithetisch gegenübergestellten Gruppe überschriftartig korrespondiert. Der Gegensatz wird zugleich durch

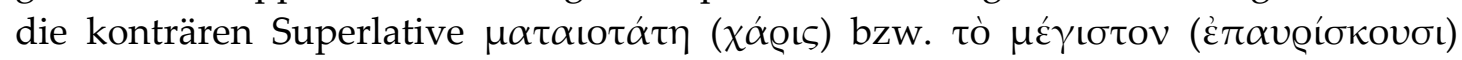
sinnfällig herausgearbeitet.

Was den Schlußpentameter anbelangt, so hat Hudson-Williams recht damit, daß $\mu \nu \tilde{\eta} \mu \alpha$ nicht im Sinne von $\mu v \eta \dot{\mu \eta} v$ gebraucht sein kann (anders etwa van Herwerden 1870: 6, van Groningen 1966 z.St. und Gerber 1970: 280). Trotzdem ist

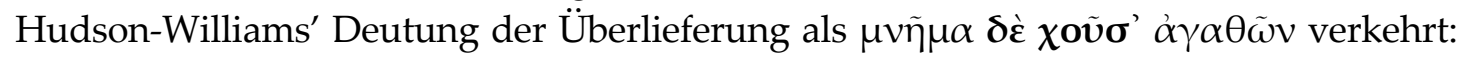
Die Worte bedeuten in ihrer herkömmlichen Form: "Als Zeichen der Erinnerung empfinden sie für die empfangenen Güter auch (über den momentanen Genuß

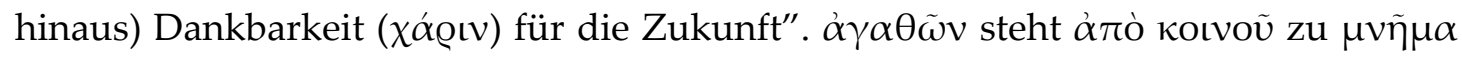

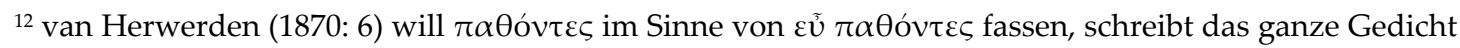
aber einem unfähigen Dichterling zu "qui hos versiculos iratis Musis composuit".
} 


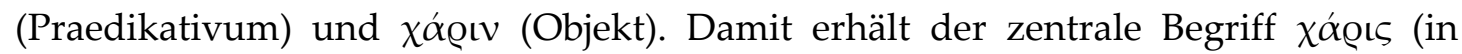

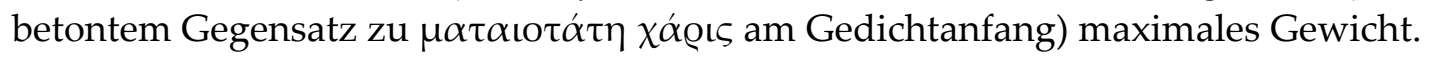

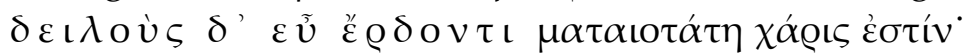

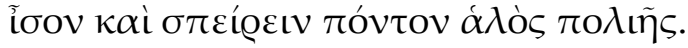

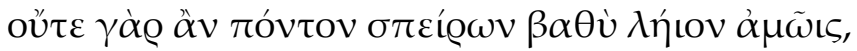

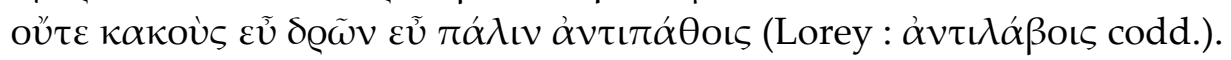

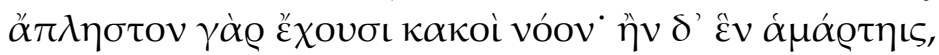

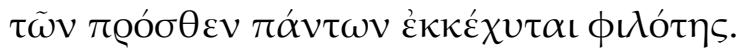

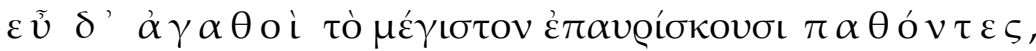

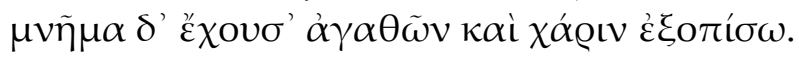

$253 \mathrm{f}$.

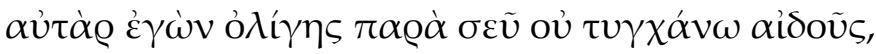

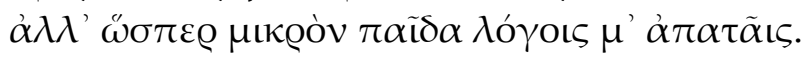

Der Hexameter leidet unter zwei Schwierigkeiten, (1) dem Hiat $\sigma \varepsilon \tilde{v}$ ov̉ und (2) der verkehrten Stellung der Negation, die (am besten in der Form oủ $\delta$ ') vor ỏ $\lambda \dot{\gamma} \gamma \eta \varsigma$ stehen sollte. Die überlieferte Wortstellung hat zu erheblichen Wirren geführt: Tarkow (1977: 99-114, bes. 114) nimmt eine gewollte Zweideutigkeit an, die einerseits "I do not chance on even slight respect from you" und andererseits auch "I chance on a good amount of respect from you" ermögliche; Gentili in einem Nachtrag zu Tarkows Aufsatz (a.a.O. 115 f.) hält nur die letztere Übersetzung für möglich und glaubt, der dem Dichter von Kyrnos erwiesene "gewaltige Respekt" werde durch den Pentameter 254 als bloße Fiktion entlarvt. Man fragt sich jedoch, worin dann die

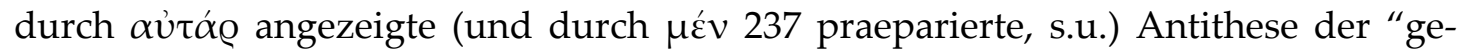
waltigen Respektserweisung" des Kyrnos zu der vorher beschriebenen, gleichermaßen gewaltigen Leistung des Dichters besteht.

Beiden oben herausgestellten Schwierigkeiten - dem Hiat und der verkehrten Stellung der Negation - trug Cobet (1858: 189) Rechnung mit seiner konjekturalen

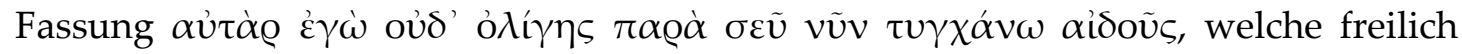
durch eine fragwürdige Krasis $(\dot{\varepsilon} \gamma \dot{\omega} \text { oủ } \delta)^{13}$ belastet ist. Einfacher und ohne metrische Schwierigkeiten wäre die Fassung La Roches, der einfach oủ durch vĩv ersetzte, womit freilich dem folgenden Anschluß mit $\dot{\alpha} \lambda \lambda^{\prime}$ der erforderliche negative Kontrast geraubt wird.

Die Wortfolge $\pi \alpha \varrho \dot{\alpha} \sigma \varepsilon \tilde{v}$ ở wird man in Anbetracht der ausgeführten Schwierigkeiten einem Interpolator zu schreiben, der einem durch Textausfall verstümmelten Text notdürftig aufhelfen wollte. Als Urfassung sei vermutet:

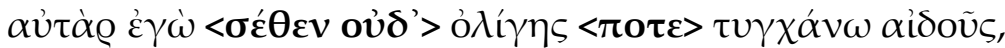

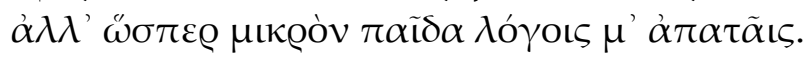

Die Form $\sigma \varepsilon \dot{\theta} \theta \varepsilon v$ ist belegt bei Theogn. 1232 (vgl. West 1974: 100)14; zur Konstruktion von $\tau v \gamma \chi \alpha ́ v \varepsilon \varepsilon v$ mit persönlichem und sächlichen Genitiv vgl. Soph. Phil. 1315 f. $\tilde{\omega} v$

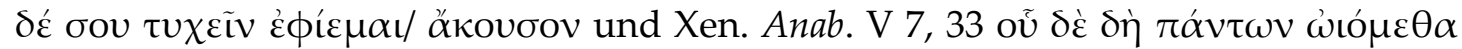

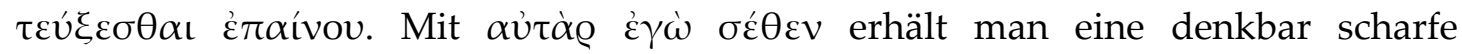

${ }^{13}$ Die bei West (1974: 86) genannten Parallelen für Krasis zwischen $\dot{\varepsilon} \gamma \omega \dot{\omega}$ und dem folgenden, vokalisch anlautenden Wort stammen sämtlich aus jambischen oder epodischen Gedichten.

${ }^{14}$ Das Gedicht wäre allerdings gemäß Vetta (1972) hellenistischer Provenienz. 


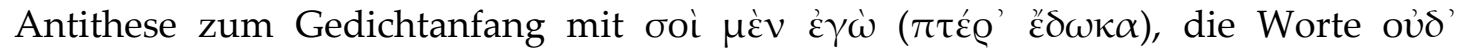

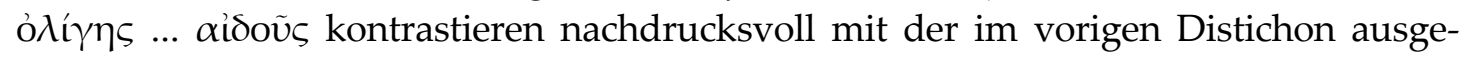
führten, dem Knaben in Aussicht gestellten a 11 s e it igen Berühmtheit ( $\pi \tilde{\alpha} \sigma$ เ

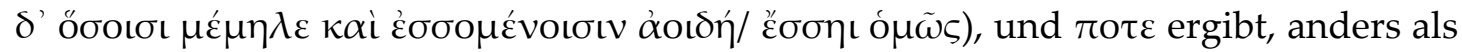
das von Cobet und La Roche eingeführte vĩv, eine klare Antithese zur Bezeichnung

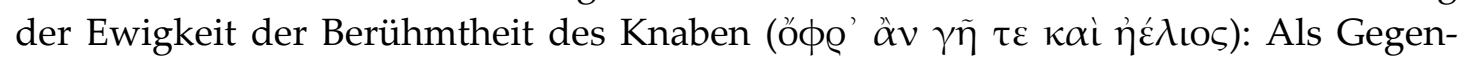
leistung für e wige alls e itige Berühmtheit erhält der Dichter nicht einmal i rge $n d w$ a $n$ e in e ge ringe Respektsbekundung. In dieser stilistisch pointierten Fassung dürfte das Schlußdistichon über jeglichen Interpolationsverdacht (Welcker, Hudson-Williams) erhaben sein, zumal Pohlenz (1932: 425) richtig darauf hingeweisen hat, daß die finale Antithese bereits durch den Anfang $\sigma o i \quad \mu \dot{\varepsilon} \nu \quad \dot{\varepsilon} \gamma \dot{\omega}$ $\pi \tau \varepsilon \dot{Q}$ ' ع̌ $\delta \omega \kappa \alpha$ praepariert ist. Die "Kurzform" des in diesem Gedicht "zerdehnten"

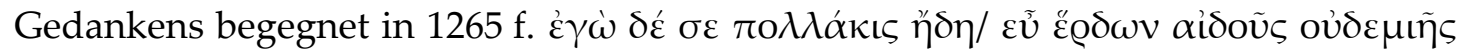
Ětuðov.

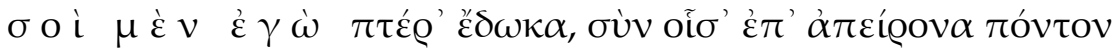

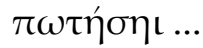

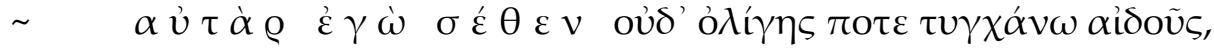

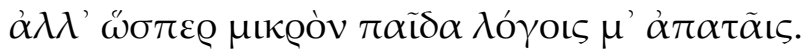

\section{(5) Influenzfehler}

Seltener läßt sich das Wirken von Influenzfehlern in den Textsegmenten der Theognideen wahrscheinlich machen.

$83-86$

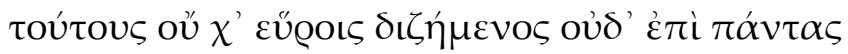

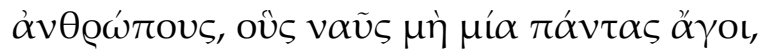

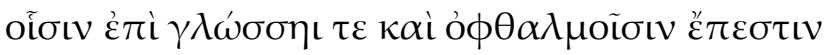

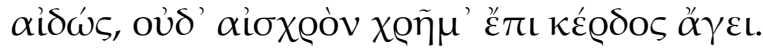

Zur Interpretation des überlieferten oủ $\chi$ als oú $\chi$ ' (van der Mey) vgl. 645 f. $\pi \alpha u ́$ govs

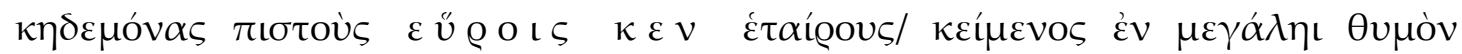
$\dot{\alpha} \mu \eta \chi \alpha$ ví

Die Schwierigkeiten im ersten Distichon liegen in der Verwendung von

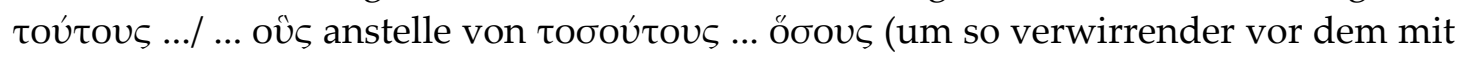
oĩotv beginnenden weiteren Relativsatz) und in der sonderbaren Stellung von $\mu \grave{\eta}$

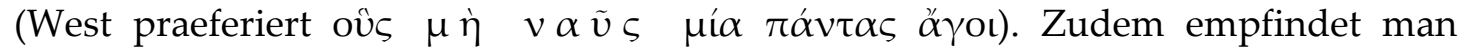

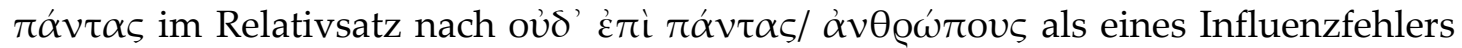
verdächtig. Besserung ergäbe sich etwa mit folgender Textfassung:

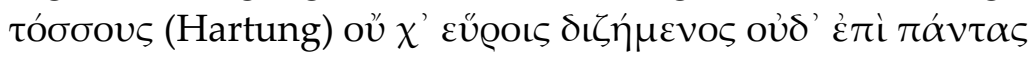

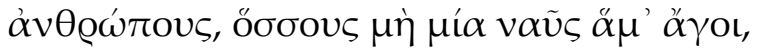

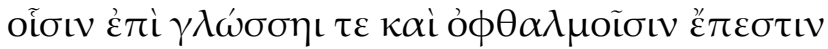

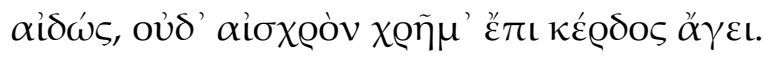

H. van Herwerden (1884: 294 f.) sieht die Negation $\mu$ í als interpoliert an und schreibt

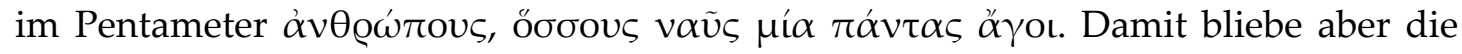
unschöne Wiederholung von $\pi \alpha \dot{v} \tau \alpha \varsigma$ bestehen, und vor allem ist zweifelhaft, ob sich 


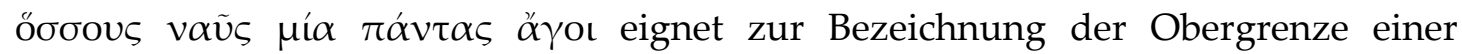
geringen Zahl von Menschen; viel eher würde man hier erwarten "soviele wie ein

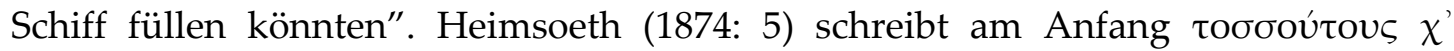

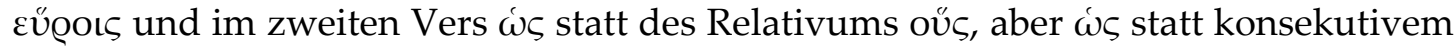
$\omega ̋ \sigma \tau \varepsilon$ scheint bei finiten Verben nicht gebräuchlich, vgl Kuehner/ Gerth II 501 Anm.

$627 \mathrm{f}$.

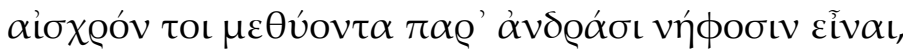

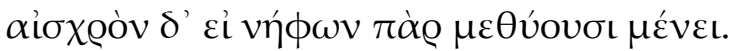

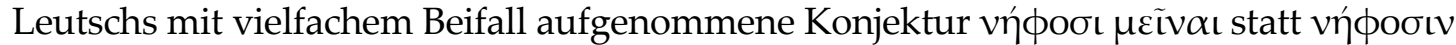
عĩvaı (1865: 19) am Hexameterende geht in die verkehrte Richtung, weil nicht der Begriff des "Bleibens" bzw. "Verharrens" erfordert ist (ganz abgesehen von dem unangenehmen Tempuswechsel $\mu \varepsilon \tilde{i} v \alpha \iota-\mu \varepsilon \dot{v} \varepsilon \iota)$, sondern einfach die Vorstellung "sich in jemandes Gesellschaft befinden" (episch $\mu \varepsilon \tau \varepsilon i \tilde{v} \alpha \iota$ cum dat.). Daher sollte

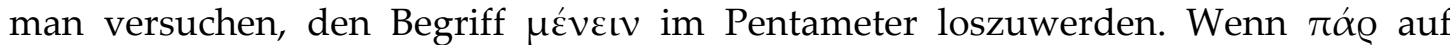
einem Influenzfehler aus dem Hexameter beruht, der ein ursprüngliches eís ("als

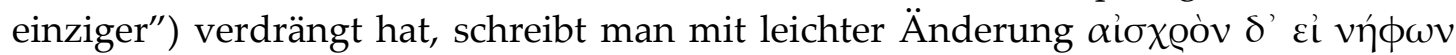

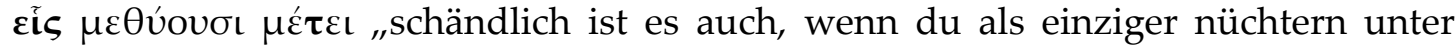
Betrunkenen bist".

(6) Lücken

Die Annahme von Lücken läßt sich häufig vermeiden (vgl. schon oben zu 53-62).

$183-192$

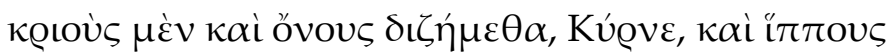

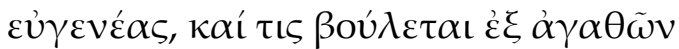

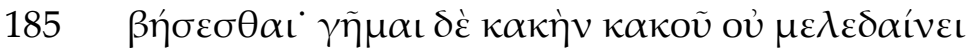

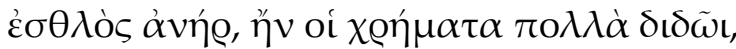

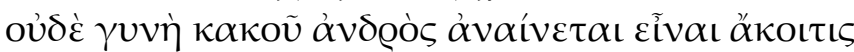

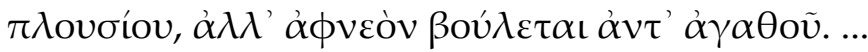

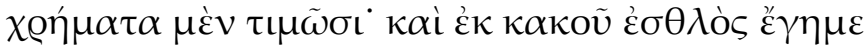

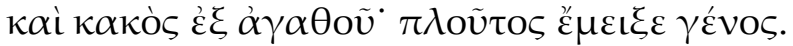

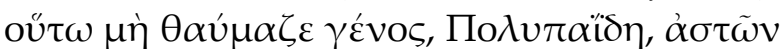

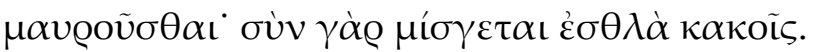

West nimmt einen frühzeitig (vor Xenophon, der hier Gewährsmann des StobaiosExzerpts ist) in der Überlieferungsgeschichte eingetretenen Textausfall nach Vers 188 an. In der Tat befremden die recht wenig besagenden Worte $\chi \varrho \eta ́ \mu \alpha \tau \alpha \mu \dot{\varepsilon} \nu$ $\tau \iota \mu \tilde{\omega} \sigma \iota$, insbesondere aber die Partikel $\mu \varepsilon ́ v$ (in der Stobaios-Überlieferung

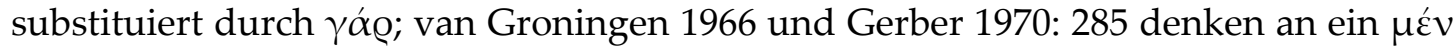
solitarium), die auf keinen passenden Gegenbegriff verweist. Ohne Lücke käme man dagegen aus, wenn man anstatt $\chi \emptyset \eta ́ \mu \alpha \tau \alpha \mu \dot{v} \nu \tau \iota \mu \tilde{\omega} \sigma \iota$ eine das Geschehen aus Sicht des oligarchischen Betrachters kommentierende, asyndetisch anschließende Sentenz 
über das Wirken des Reichtums vor sich hätte, die dann exakt mit dem folgenden

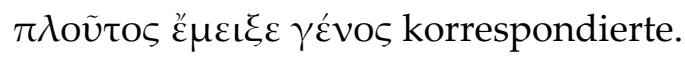

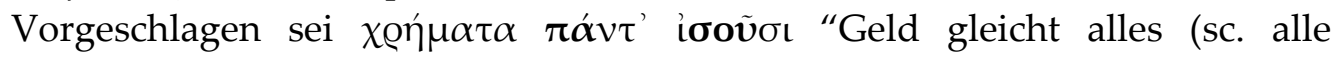
natürlichen Unterschiede) aus". Zu iooṽv mit langer Anfangssilbe vgl. Hom. Od. $\eta$

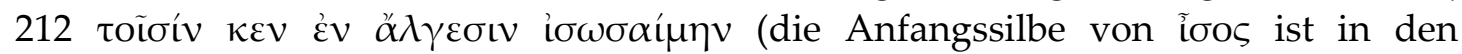
Theognideen mit Ausnahme von Vers 678 grundsätzlich lang, 82. 106. 224. 271. 544. 719, vgl. Sitzler 1885: 10), zum pluralischen Praedikat neben dem Subjekt im Neutrum Plural (insbesondere im Zusammenhang der Vereinzelung mehrerer Fälle) vgl. die Belege bei Kuehner/ Gerth I 64-66.

$1381-1384$

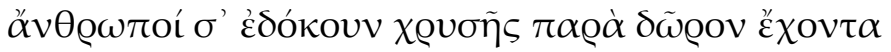

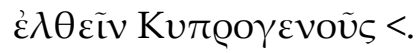

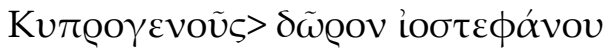

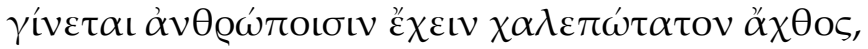

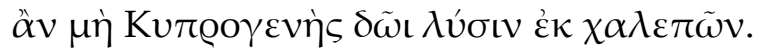

West nimmt die von Bekker angenommene Lücke entsprechend der communis opinio ${ }^{15}$ in seinen oben reproduzierten Text. Alternativ kann man mit dem ersten Distichon zurande kommen, wenn man annimmt, daß sich hinter dem zweiten $\delta \tilde{\omega} \varrho o v$ im Pentameter (Influenzfehler aufgrund desselben Wortes im Hexameter) diejenige Eigenschaft des Knaben verbirgt, die als Geschenk vonseiten der Liebesgöttin bezeichnet

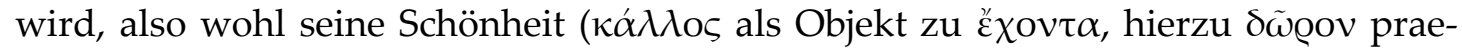

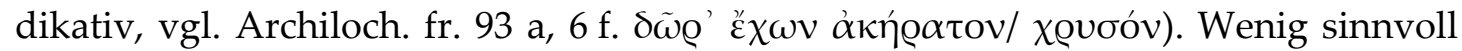
ist demgegenüber das Verfahren von Carrière, der $\beta \omega \mu$ óv (Richtungsakkusativ zu $\dot{\varepsilon} \lambda \theta \varepsilon \tilde{\varepsilon} v)$ schreibt für das zweite $\delta \tilde{\omega} \varrho o v$ und die Verse $1381 \mathrm{f}$. als selbständiges Gedichtfragment betrachtet.

Die Vermutung "der Menschen", hinter der Schönheit des angesprochenen Knaben stecke ein Geschenk der Liebesgöttin, wird jedoch sehr bald in Frage gestellt, wenn sie sich in ihn verlieben und seine nähere Bekanntschaft machen: Dann zeigt sich, daß die Liebe zu ihm ein $\chi \alpha \lambda \varepsilon \pi \omega \omega \tau \alpha \tau o v ~ \alpha ̋ \chi \emptyset o \varsigma$ ist, von dem wiederum nur die Liebesgöttin durch ihre Gnade befreien kann. Dieser Gedanke ergibt sich mit folgender Herstellung:

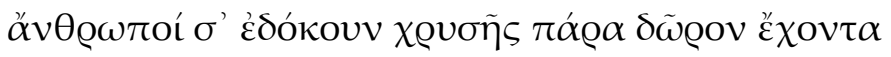

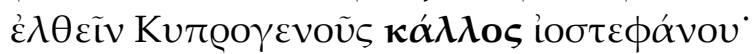

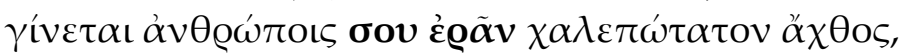

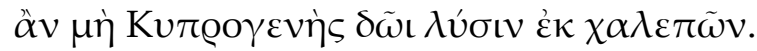

Das Asyndeton zwischen den beiden Distichen erklärt sich letztlich aus einem Ursache-Wirkungs-Verhältnis (vgl. Kuehner/ Gerth II 342 f.): Die Enttäuschung der liebenden Menschen ist eine (mittelbare) Folge der erotischen Ausstrahlung des Angesprochenen.

\footnotetext{
${ }^{15}$ Anders etwa Heimsoeth 1874: 15.
} 
An einer anderen Stelle ist die Annahme einer Lücke dennoch hilfreich.

$667-671$

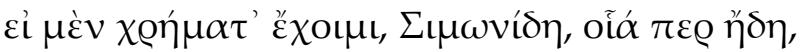

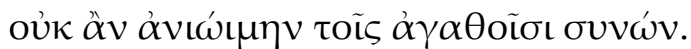

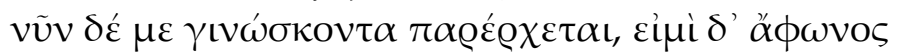

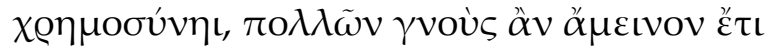

oúvยK $\alpha \ldots$

In Vers 669 wird ein Subjekt zu $\pi \alpha \varrho \varepsilon ́ \varrho \chi \varepsilon \tau \alpha \iota$ (das zugleich Objekt zu $\gamma \mathrm{t} \omega \omega ́ \sigma \kappa o v \tau \alpha$ sein sollte) gesucht, das kaum in $\chi \varrho \eta ́ \mu \alpha \tau \alpha$ gefunden werden kann (so Hudson-

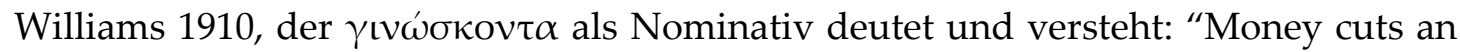
old acquaintance"). Ein solches ließe sich relativ leicht mit folgender Textfassung gewinnen:

$\nu \tilde{v} \nu \delta \varepsilon \mu^{\prime}, \hat{\alpha} \gamma\llcorner\nu \omega ́ \sigma \kappa \omega, \tau \dot{\alpha} \pi \alpha \varrho \varepsilon ́ \varrho \chi \varepsilon \tau \alpha \iota . .$.

Doch entschieden gegen diese Lösung (wie auch gegen Hudson-Williams' Interpretation) spricht die enge Parallele $419 \mathrm{f}$.:

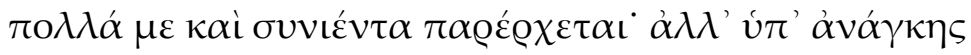

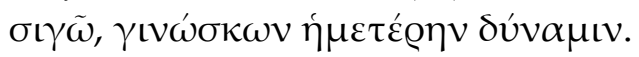

Im Lichte dieser Parallele empfiehlt sich die Annahme, daß nach vĩv $\delta \varepsilon ́$ eine nähere Erörterung der tatsächlichen momentanen wirtschaftlichen und gesellschaftlichen Lage des Sprechers ausgefallen ist und erst im folgenden Distichon zu den speziellen Konsequenzen für die politische Beratung übergegangen wird:

$v \tilde{v} v \delta \varepsilon \dot{\varepsilon}<$

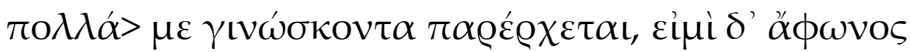

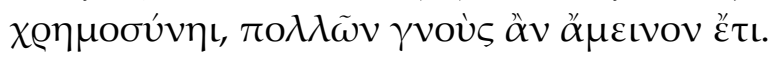

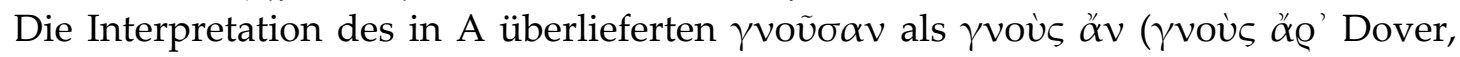

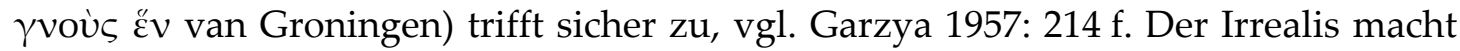
den Gedanken der Verhinderung durch äußere Umstände erst vollends deutlich: "ich wüßte Besseres als viele, sc. wenn ich nicht durch meine Mittellosigkeit am Sprechen gehindert wäre". Fraglich erscheint allerdings, ob der Begriff $\pi \mathrm{o} \lambda \lambda \tilde{\omega} \nu \ldots$

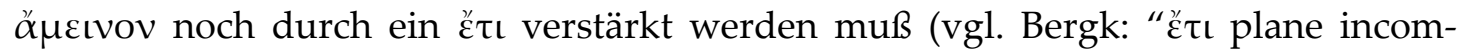

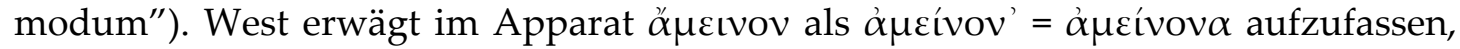
und mit dieser Deutung kann man eine leichte Änderung verbinden, die dann einen höchst praegnanten Gegenbegriff zu ő $\phi \omega v o \varsigma$ schafft (der im Prinzip schon durch Wests Formulierung "though, if I were speaking, I would have given a v e r d i c t yet better than many people" (1974: 157) antizipiert wird):

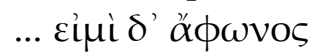

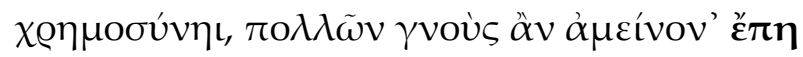

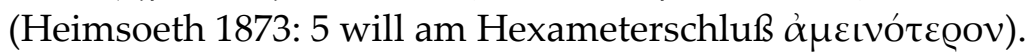

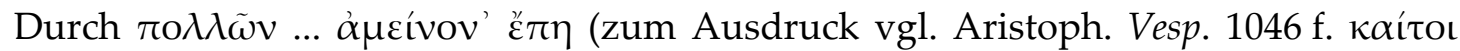

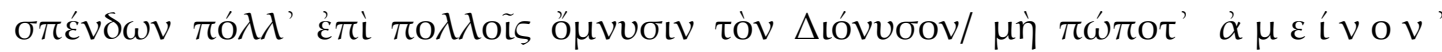

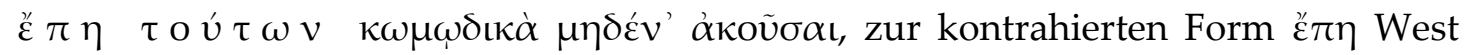

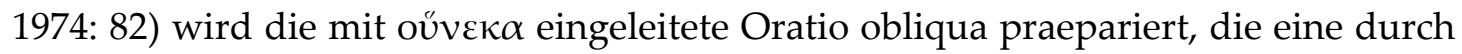


Bildsprache verrätselte politische Botschaft enthält und abgeschlossen wird durch

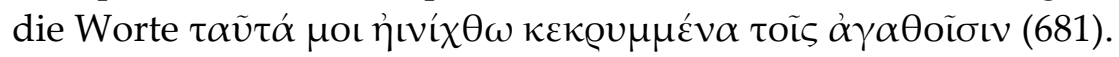

\section{(7) Umstrittene Gedichtabgrenzungen und Umstellungen}

Eher selten ist die Abgrenzung der einzelnen Exzerpte und die Reihenfolge der Distichon umstritten.

$511-522$

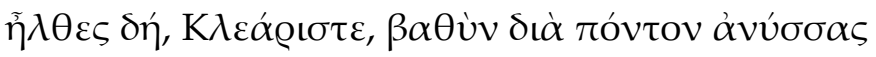

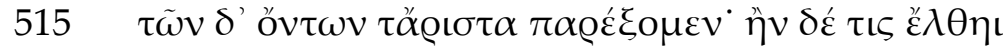

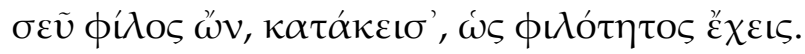

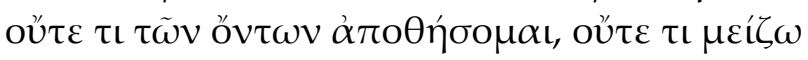

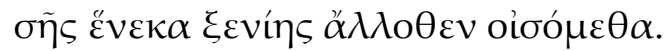

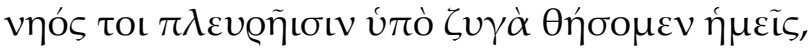

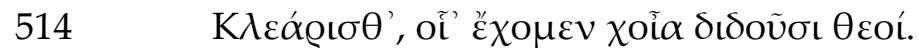

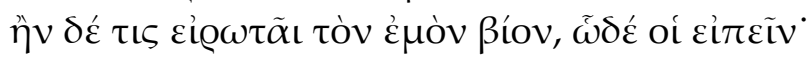

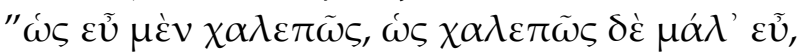

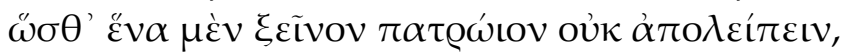

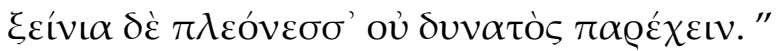

Die Versetzung Wests (1974: 156) dient dem Zweck, die Bewirtung des Freunds gemäß dem Vorbild von Odysseus' Aufenthalt bei den Phaiaken der Verproviantierung des Rückwegs voranzustellen. Sie beruht jedoch auf einer höchst ungewissen, nicht weiter hinterfragten Voraussetzung: daß nämlich die oben ausgeschriebenen Verse 511-522 überhaupt ein geschlossenes Gedicht bilden.

Die Schiffahrtsthematik findet sich ausschließlich in den Versen 511-514, ebenso der Name Klearistos. Diese Verse fügen sich ohne weiteres zu einem kurzen Gedicht zusammen, in welchem der als arm dargestellte Sprecher seinen zu Schiff einlaufenden, ebenso armen Gastfreund notdürftig verproviantiert. Der Gedanke erscheint mit dem durch bekräftigendes toı bezeichneten Aufschluß der Erwartung vollkommen geschlossen, wie es Sitzler (1878: 17) durch seine Paraphrase zum Ausdruck bringt: "venisti ad me, exspectans, ut te excipiam: sane tibi apponam, quae habeo". Nur muß man statt "excipiam" einsetzen "adiuvem" und statt "apponam"

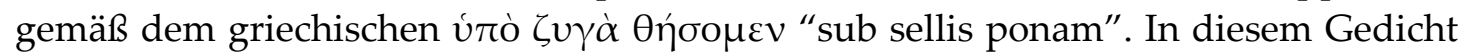
ist so wenig an ein Gastmahl gedacht wie in den folgenden Versen an eine Schifffahrt.

Die Verse 515-522 sind mit Hartung als ein separates Gedicht anzusehen, das mit dem vorigen (511-514) nur durch das allgemeine Thema einer Freundschaft in begrenzten wirtschaftlichen Verhältnissen verbunden ist. Das $\delta$ ' zwischen $\tau \tilde{\omega} v$ und ǒv $\tau \omega \nu$ könnte dann von einem Excerptor stammen und nur dem Zweck dienen, einen nicht vorhandenen Anschluß der Verse 515 ff. an 511-514 vorzutäuschen.

Entscheidend für das Verständnis dieses zweiten Gedichts ist das Distichon $515 \mathrm{f}$. Was soll die Aussage "Wenn ein Freund von dir gekommen ist, so liege entsprechend dem Rang deiner Freundschaft (zu mir) nieder" (West: "and if you 
have a friend to bring, make yourself at home")? Genaugenommen wird dem Angesprochenen nicht die Möglichkeit eingeräumt, einen Freund mitzubringen (was Wests Ausdruck "if you have a friend to bring" zu implizieren scheint), sondern

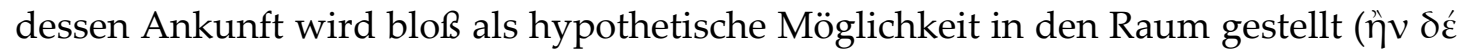

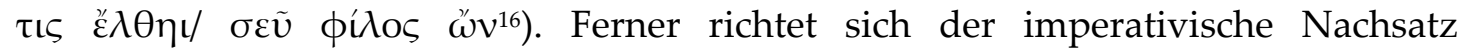

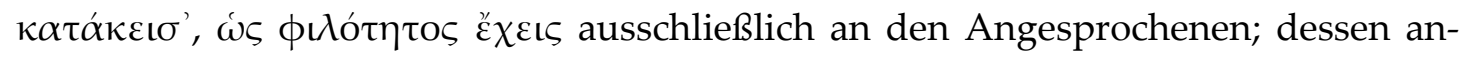
gekommener Freund wird in keiner Weise in die Einladung mit einbezogen.

Dieser vermeintliche Mißstand veranlaßte die Konjekturen $\kappa \alpha \tau \alpha \kappa \varepsilon i ́ \sigma \theta^{\prime}(\omega)$ (Boelte, Allen (1930: 189) und Howald prosodisch bedenklich, die beiden erstge-

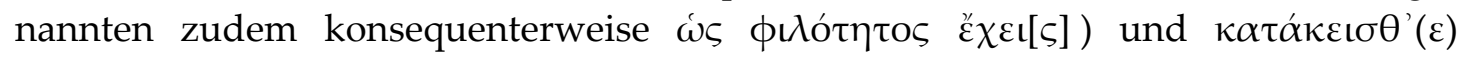
(Dover), vgl. van Groningen und Young z.St. Weitere ziemlich groteske Versuche, den Freund in die Einladung miteinzubeziehen, findet man in Bergks Apparat ad loc. Gemäß dem Schlußdistichon ist es aber klar, daß der Sprecher überhaupt nicht gewillt ist, einen solchen "Freund zweiter Ordnung" mitzubewirten. Der Sinn der

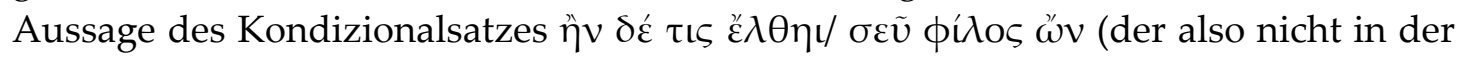
Miteinladung des Dritten liegen kann) bleibt weiterhin rätselhaft. Am konsequentesten wäre es noch, diese Vorstellung einer Mit-Einladung des Dritten vollständig zu eliminieren durch eine Beseitigung des Verbs $\kappa \alpha \tau \alpha \kappa \varepsilon \tilde{\imath} \sigma \theta \alpha \mathrm{l}$, wie sie etwa Sitzler

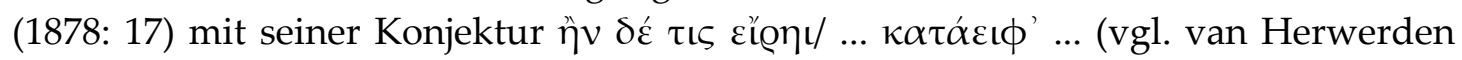

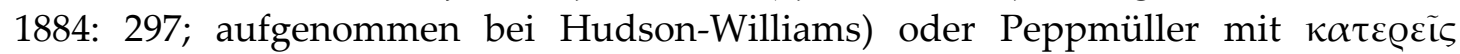
erreicht.

Ein ähnlicher Sinn (der Dritte nicht als zusätzlicher Gast, sondern als die Freundschaft zwischen Sprecher und Angeredetem beurteilende Instanz) läßt sich jedoch auch mit erheblich geringerem Aufwand erreichen. Mit Änderung eines

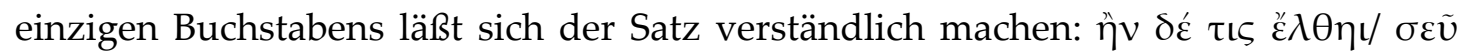

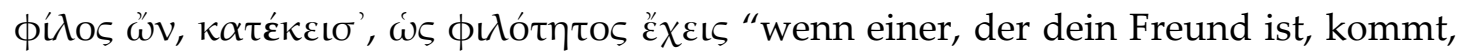
so lagest du (wie er bezeugen wird) nieder, wie es deiner Freundschaft mit mir

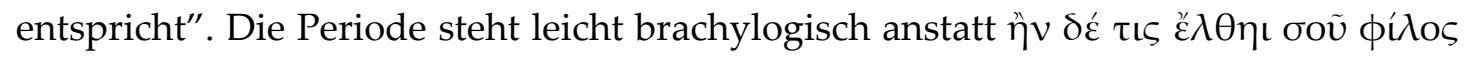

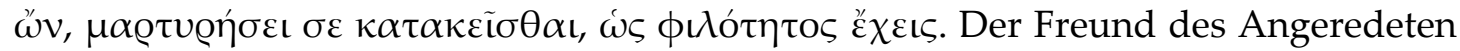
ist nicht als zusätzlicher Gast gedacht, sondern hat die Funktion, darüber Zeugnis abzulegen, daß der Angeredete vom Sprecher in einer Weise bewirtet wurde, wie es dem seit früher existierenden Freundschaftsverhältnis zwischen beiden entspricht. Er wird in der Zukunft sagen können, daß der Angeredete vom Sprecher angemessen bewirtet wurde.

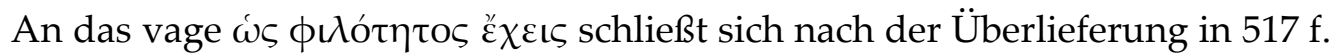
ein konkreter Hinweis an, wie die angemessene Bewirtung aussehen soll: Sie soll dem Angeredeten weder etwas von den begrenzt vorhandenen Ressourcen vorenthalten noch den Sprecher in die Lage versetzen, weitere Mittel von auswärts aufnehmen zu müssen.

In den beiden Schlußdistichon wird dann ein genaueres, fast ein wenig penetrantes Nachfragen des Gastfreunds des Angeredeten geschildert. Dieser gibt sich nicht damit zufrieden, Zeugnis abzulegen über die angemessene Bewirtung des Angeredeten beim Sprecher, sondern sucht Genaueres über die (wie in $517 \mathrm{f}$. angedeutet) begrenzten Mittel des Sprechers zu eruieren. Vers 519 ist mit van

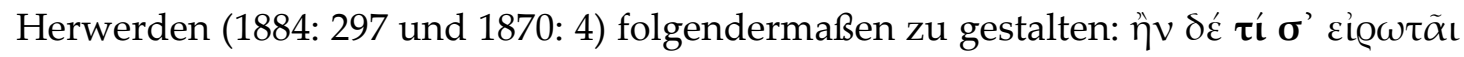

\footnotetext{
${ }^{16}$ Rosati (2000: 337-340) vergleicht hierzu Ov. trist. I 1, 17-19 Siquis, ut in populo, nostri non immemor illi, I Siquis, qui, quid agam, forte requirat, erit:/ Vivere me dices, salvum tamen esse negabis.
} 


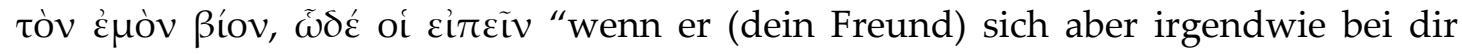
nach meinen Lebensumständen erkundigt, so sag ihm Folgendes ...".

Die auf diese Weise gewährte Information läuft auf die Schlußpointe hinaus, daß der Sprecher gerade so viel besitzt, um den Angesprochenen, einen alten Gastfreund, angemessen bewirten zu können, keinesfalls aber soviel, daß noch zusätzlich andere (wie dessen hinzugekommenem Freund) empfangen kann. Der angekommene Freund des Angesprochenen wird damit letztlich als Schmarotzer entlarvt, der nur das Ziel verfolgte, selbst am Gastmahl teilnehmen zu können. Ihm gegenüber macht der Sprecher unmißverständlich klar, daß er ihn nicht bewirten wird. Die Gesamtaussage des Gedichts ist: "Ich werde dich, meinen alten Gastfreund, unter Aufwendung der mir zur Verfügung stehenden Mittel angemessen bewirten, wovon sich ein hinzukommender Freund gerne überzeugen darf. Er soll aber nicht hoffen, selbst mitessen zu können".

Sehr gut vereinbar wäre diese Deutung mit der ebenfalls von van Herwerden a.a.O. vorgeschlagenen ${ }^{17}$ Versetzung der Distichen 515 f. und 517 f.: Dann würde der programmatischen Formulierung des oekonomischen Prinzips der Bewirtung (517 f.) die Auseinandersetzung mit dem hypothetisch gesetzten Fall der Ankunft eines Freunds des Angesprochenen (515 f., 519 f.) folgen. Insbesondere schließt sich an die

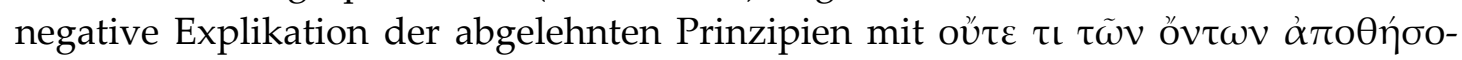

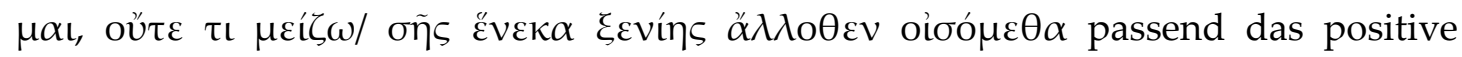

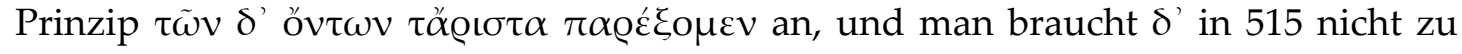
tilgen. Im ganzen:

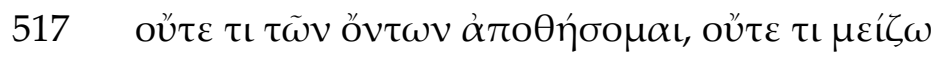

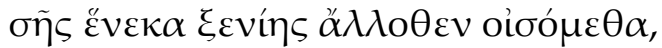

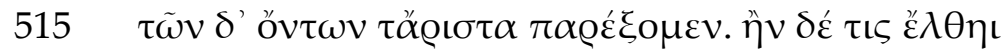

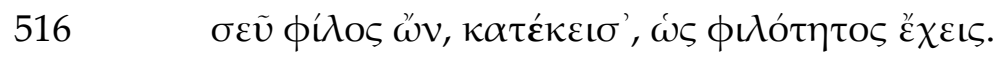
ทे

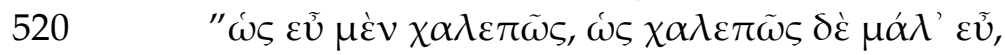

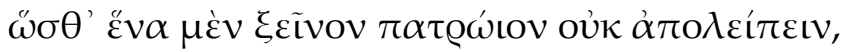

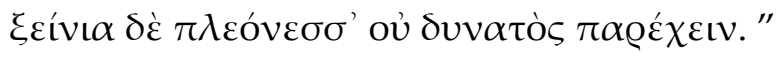

(8) Rekurs auf archaische Vorbilder

Hilfreich ist gelegentlich der Rekurs auf archaische Vorbilder, die in einzelnen Textsegmenten vom Dichter der Theognideen herangezogen werden:

203-208

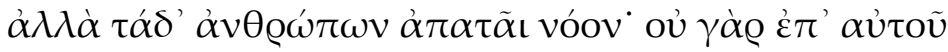

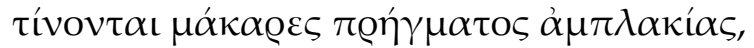

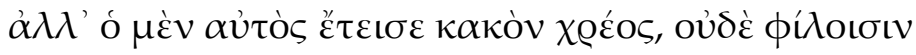

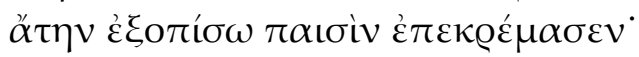

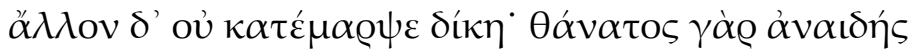

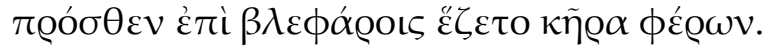

${ }^{17}$ Vgl. Hudson-Williams z.St. und Garzya 1957: 211-213. 
Der Text kommt in Ordnung, wenn man (wie West zwar im Apparat erwägt, aber in 1974: 151 f. verwirft) in Vers 205 statt oủ $\delta \dot{~ m i t ~ p ~ o ̂ \varsigma ~ \delta \varepsilon ́ ~ s c h r e i b t ~ u n d ~ d a n n ~ i n ~ V e r s ~} 207$

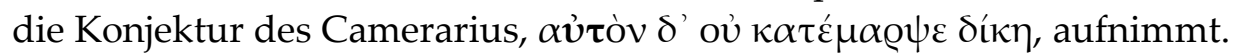

Der Verfasser des vorliegenden Gedichts modifiziert das Drei-Stufen-Modell des Solon zu einem Zwei-Stufen-Konzept:

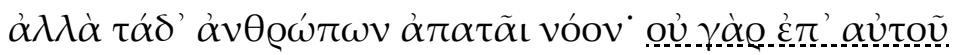

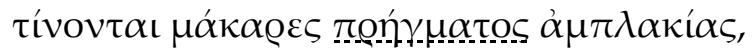

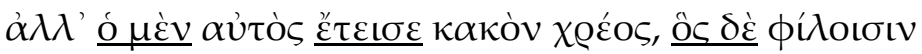

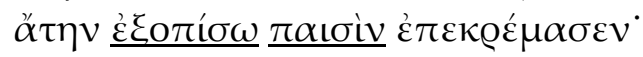

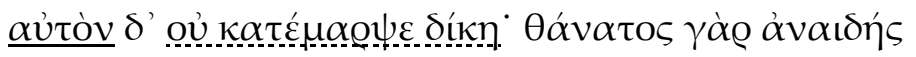

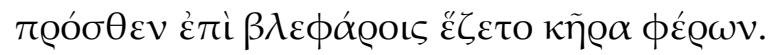

$\sim$ Solon fr. 13, 25-32

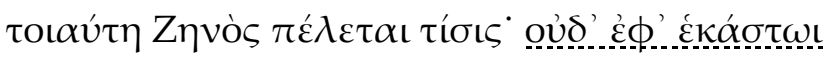

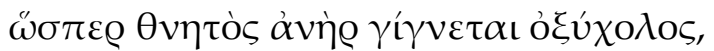

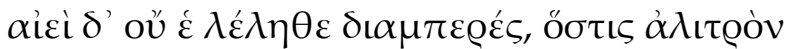

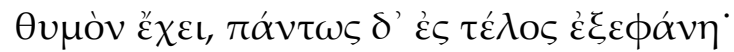

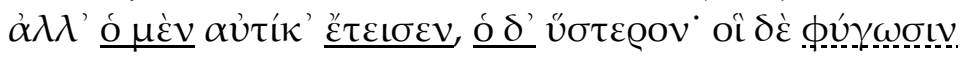

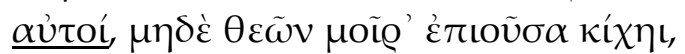

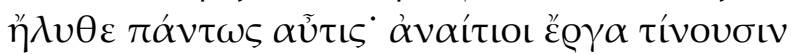

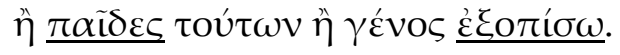

Bei Solon existiert noch die Möglichkeit, daß die Götter ein Verbrechen sofort bestrafen; es heißt nur, daß sie nicht in jedem Fall (oủ ' ' Alternative zu einer sofortigen Bestrafung ist eine spätere, die entweder noch den Übeltäter selbst in seinem späteren Leben oder aber seine unschuldigen Nachkommen trifft.

In den Theognideen heißt es dagegen, daß die Götter grundsätzlich nicht sofort

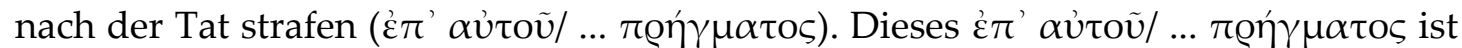
Jacobs' den erforderten Sinn treffende Konjektur, die auch in der separaten Behandlung des vorliegenden Gedichts bei Huijing \& van Raalte (1981: 13 f.) akzeptiert wird (noch weiter ging Brunck mit '̇ं $\pi^{\prime} \alpha \hat{v} \tau o \tilde{\iota} \varsigma / \ldots \pi \emptyset \eta ́ \gamma \mu \alpha \sigma \iota v$ ), für

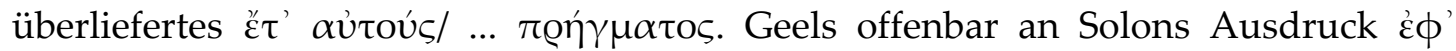

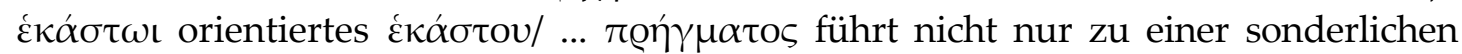

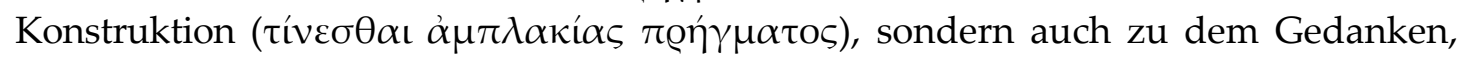

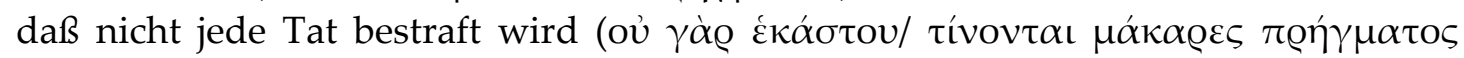

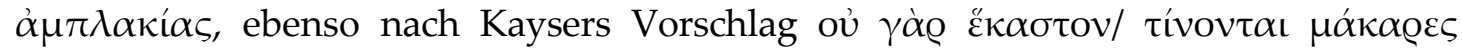
$\pi \varrho \eta ́ \gamma \mu \alpha \tau o \varsigma \alpha \dot{\alpha} \mu \pi \lambda \alpha \kappa i ́ \eta \varsigma)$, also ein Verbrechen auch völlig unbestraft bleiben kann was weder bei Solon noch in den Theognideen vorgesehen sein kann. Auch

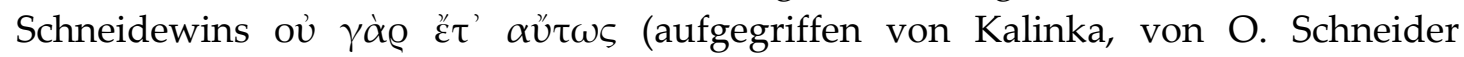

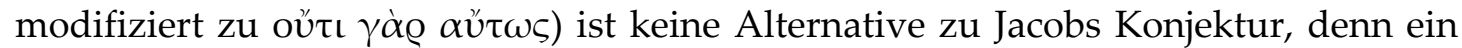
$\alpha u ̛ \tau \omega \varsigma$ in dem Sinne "eodem modo" benötigte als Gegenbegriff unbedingt den Ausdruck "j e d e Verfehlung", ganz abgesehen von dem wieder müßig allein-

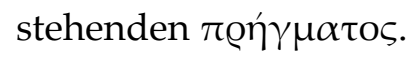


Für den Theognideen-Dichter gibt es anders als für Solon keine sofortige, sondern nur eine spätere Bestrafung, die sich entweder am noch lebenden Übeltäter oder nach dessen Tod an seinen Nachfahren vollzieht.

$557-560$

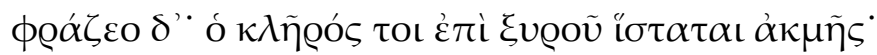

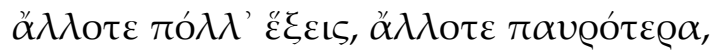

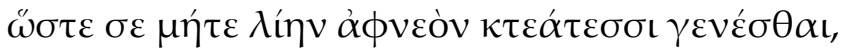

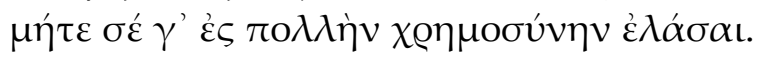

Die oben gedruckte Version am Anfang ist Wests Konjektur (1974: 156). Überliefert

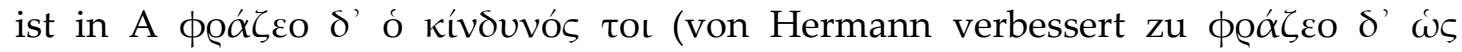

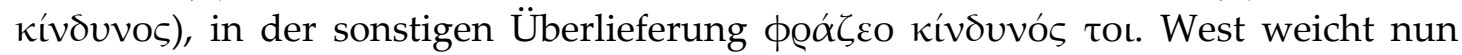
von der communis opinio (die letztere Version für authentisch ansieht) ab und

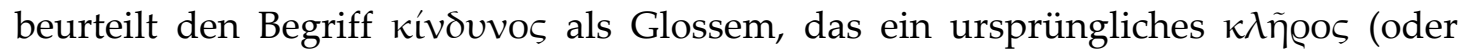
$\kappa \tau \tilde{\eta}$ vos) aus dem Feld geschlagen habe.

Fraglich erscheint aber, ob die Aussage "dein Erbe (oder "dein Besitz") steht

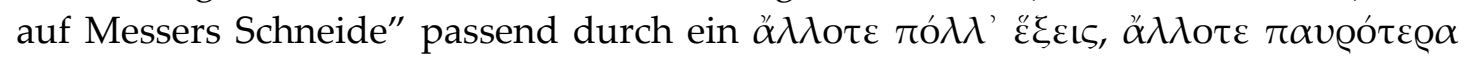
fortgeführt wird, welches dann seinerseits zu einer moralisierenden Aufforderung überleitet, in Sachen Besitz die aurea mediocritas zu erstreben. Denn der Vers 557 erweckt in Wests Version den Eindruck, als bestehe eine unmittelbare, akute Bedrohung für den Besitz des Angeredeten und nicht etwa nur die jeden Menschen bedrohende Unsicherheit, irgendwann einmal Mangel ertragen zu müssen. Der griechischen Begriff kívouvos schließt nun aber diese existentielle Unsicherheit (anders als das deutsche "Gefahr", das immer auf etwas Akutes hindeutet) durchaus mit ein ${ }^{18}$, wie 585-590 (aus Solon, fr. 13, 65-70) dartut:

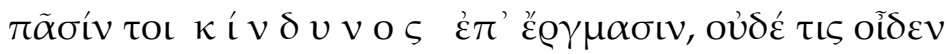

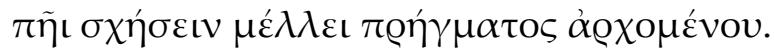

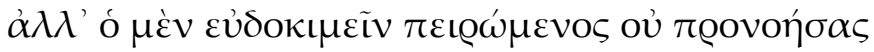

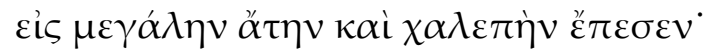

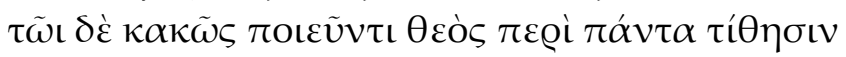

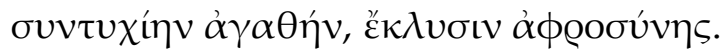

Für die "existentielle Unsicherheit" hinsichtlich der Besitzverhältnisse scheint also gerade das von West verbannte kívঠuvos der richtige Begriff zu sein. Freilich hat West andererseits recht, wenn er sagt: "Something at risk may be said to be غ̇ंì $\xi$ vอov $\dot{\alpha} \kappa \mu \tilde{\eta} \varsigma$; danger itself cannot". Unter Zugrundelegung der Vulgata erhält man mit einer leichten Textänderung:

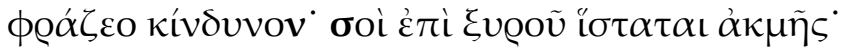

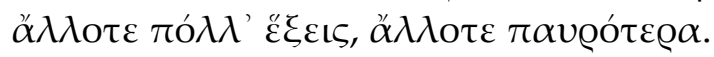

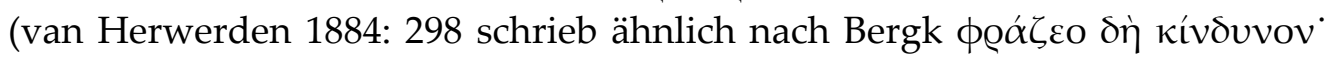

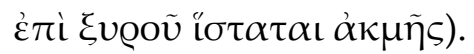

Die so hergestellte Textfassung mit der unpersönlichen, durch einen syntak-

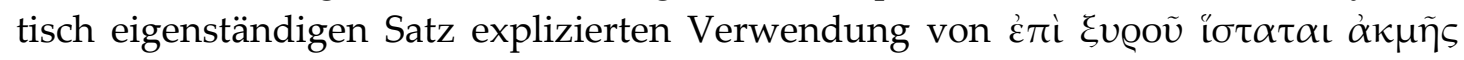
unterscheidet sich nicht erheblich von dem homerischen Vorbild Il. K 173 f.:

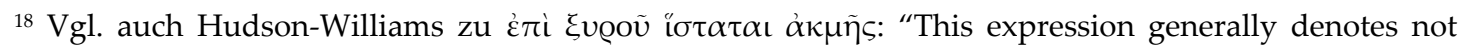
danger but uncertainty". 


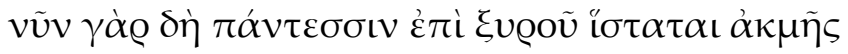

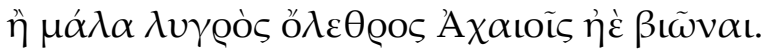

Diese Konstruktion wird treffend analysiert von Leaf z.St.: "i $\sigma \tau \alpha \tau \alpha \iota$ is really an impersonal verb, and the substantive of $\lambda \varepsilon \theta 0 o \varsigma$ is not added in a very strict construction. Logically, the idea is 'the state of all is on the razor's edge (balancing) between destruction and safety' ".

897-900

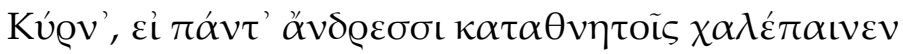

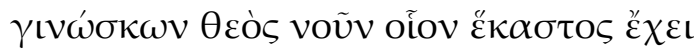

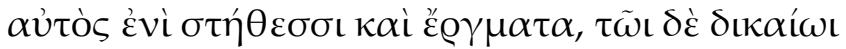

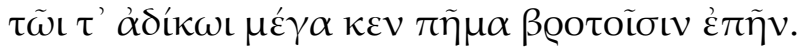

Zur textkritischen Behandlung des äußerst schwierigen Vierzeilers (vgl. West 1974: $160 \mathrm{f}$.) sollte vor allem das homerische Vorbild Il. П 385-388 (zitiert bei HudsonWilliams) herangezogen werden:

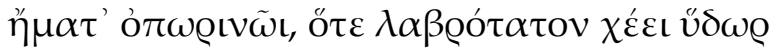

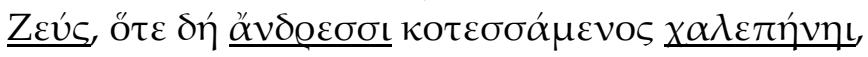

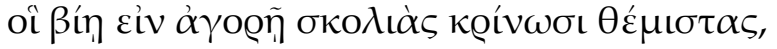

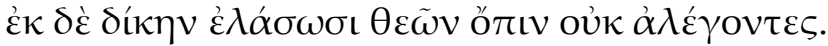

Demnach wird man für das syntaktisch unverständliche $\omega \varsigma$ in Vers 898 eher mit Bergk ein konkretes Zzús als mit West l.c. ein allgemeines $\theta \varepsilon o ́ \varsigma$ einsetzen. Vgl. auch Solon fr. 13, $25 \mathrm{f}$.

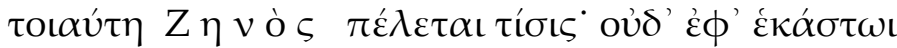

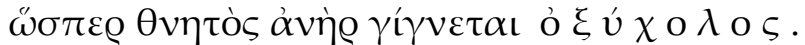

Eine Schwierigkeit ist aủtós am Anfang von Vers 899. Damit der auf voũv bezügliche Relativsatz eine ordentliche Antithese $\mathrm{zu}$ dem im folgenden gemäß

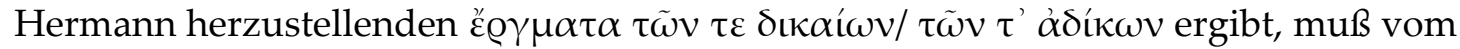
voũs "im Inneren" im Gegensatz zu den sich nach außen bekundenden Taten die Rede sein: Gemäß der schon bei Bergk aufgenommenen Variante eines cod. deterior

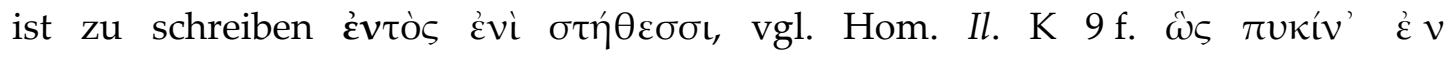

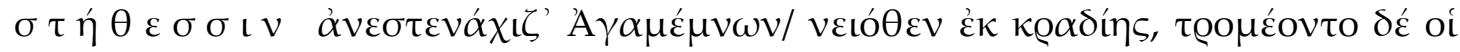

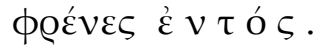

Im ganzen:

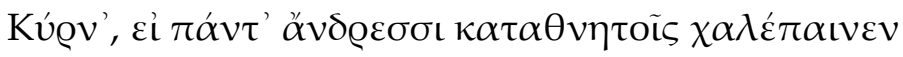

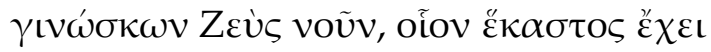

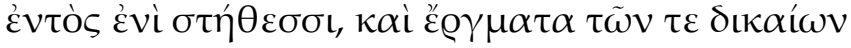

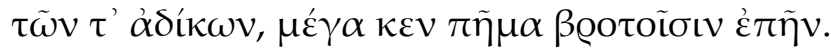

1007-1012

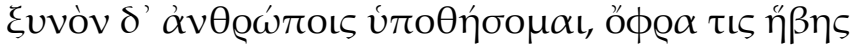

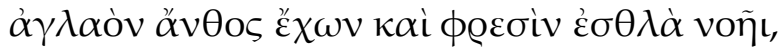

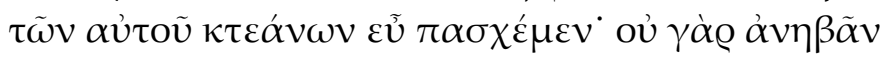

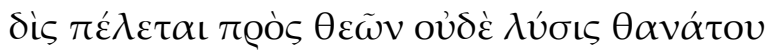

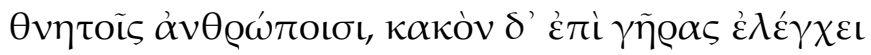

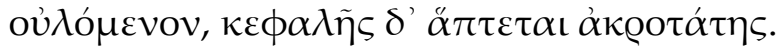




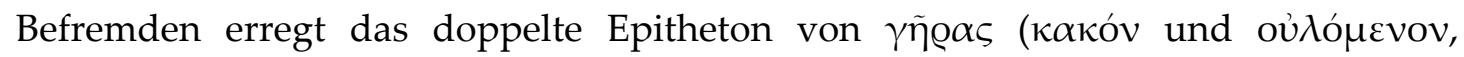
letzteres gestützt durch $1021 \mathrm{f}$.). Pierson änderte $\dot{\varepsilon} \lambda \dot{\varepsilon} \gamma \chi \varepsilon \mathrm{s}$ in $\dot{\varepsilon} \pi \varepsilon \dot{\varepsilon} \gamma \varepsilon \mathrm{\varepsilon}$, Hartung in

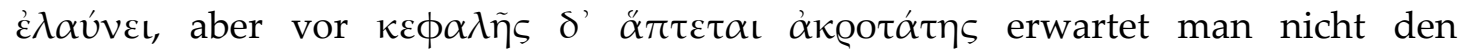
Gedanken "das Alter drängt zum Übel", sondern "das Alter widerlegt den, der (scheinbar) noch jugendlich schön ist, es berührt ihn (zunächst) an der Spitze des Kopfes (mit grauen Haaren)". $\dot{\varepsilon} \lambda \varepsilon ́ \gamma \chi \varepsilon \iota$ wird als Praedikat zu $\gamma \tilde{\eta} \varrho \alpha \varsigma$ erst wirkungsvoll durch einen Gegenbegriff, der pointiert jugendliche Schönheit dagegenstellt (vgl.

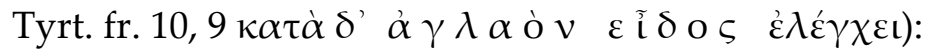

... oủ $\gamma \dot{\alpha} \varrho \dot{\alpha} v \eta \beta \tilde{\alpha} v$

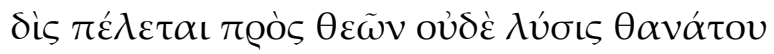

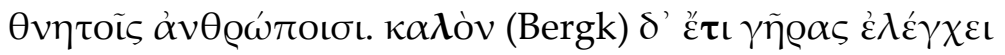

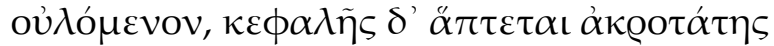

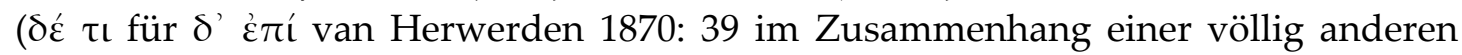

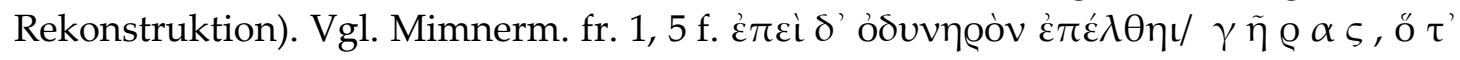

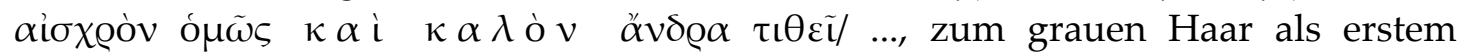
Zeichen des Alterns eines sonst noch jugendlich schönen Menschen hymn. Ven. $228 \mathrm{f}$.

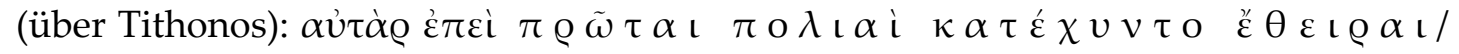

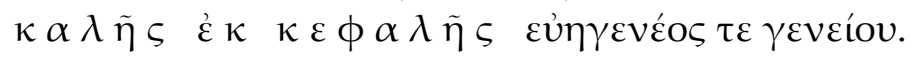

\section{$1135-1138$}

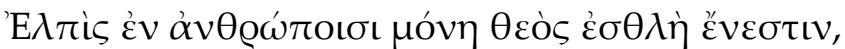

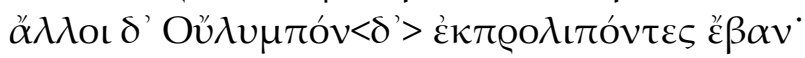

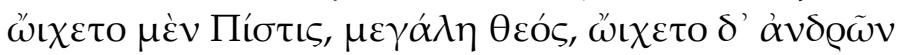

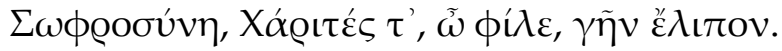

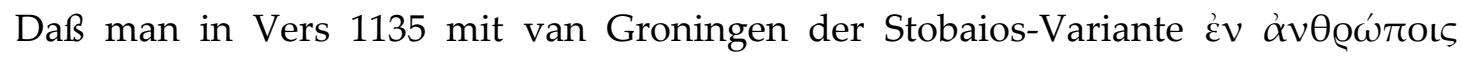

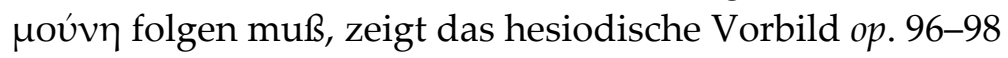

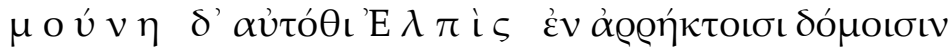

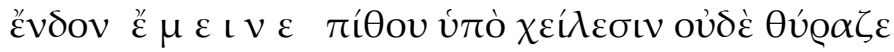
$\dot{\varepsilon} \xi \dot{\varepsilon} \pi \tau \eta \ldots$

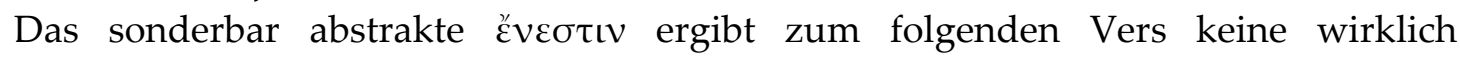

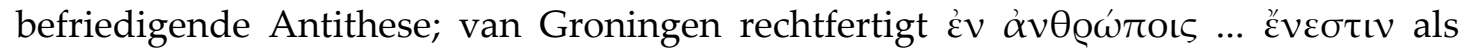
Beschreibung eines psychischen Vorgangs, aber dieser Gedanke scheint gegenüber dem “Bei-den-Menschen-Verbleiben" (im Gegensatz zu $\gamma \tilde{\eta} v \pi 00 \lambda ı \pi o ́ v \tau \varepsilon \varsigma ~ \varepsilon ̌ \beta \alpha \nu$ [s.u.] im folgenden Vers) allenfalls nebensächlich vorhanden zu sein. Gemäß Hesiod wird

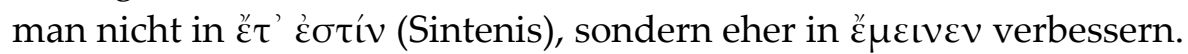

Im folgenden Vers hat Camerarius' Einfügung Oú $\lambda v \mu \pi o^{\prime} v<\delta>$ geringe

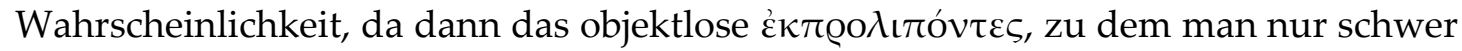
aus $\alpha \dot{v} \theta \varrho \omega ́ \pi$ oı den Akkusativ ergänzen kann, allein stehen bleibt. Zu einem formal

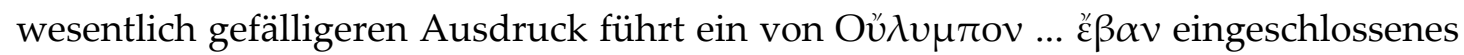

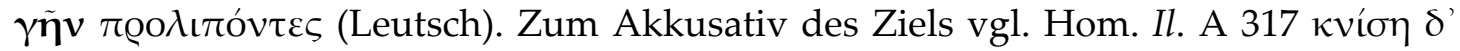

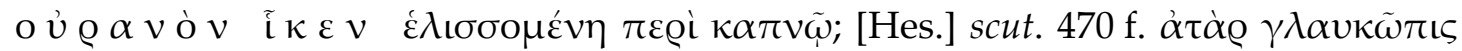

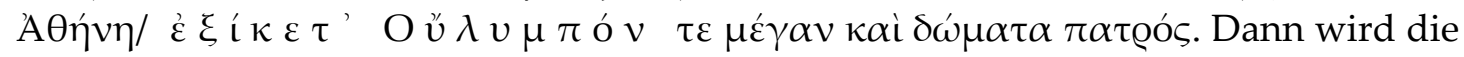
Aufzählung eingeschlossen durch die korrespondierenden Ausdrücke $\gamma \tilde{\eta} v$

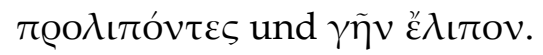


(9) Rekurs auf spätere Imitationen oder Parallelen

Andererseits können auch Imitationen oder motivische Parallelen aus späterer Dichtung hilfreich sein.

1299-1304

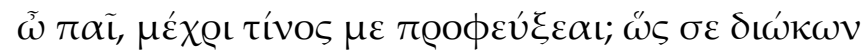

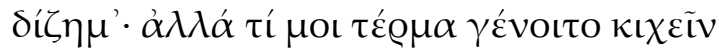

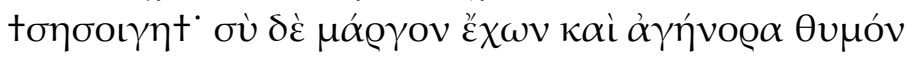

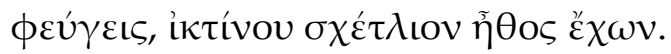

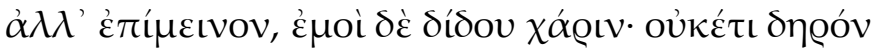

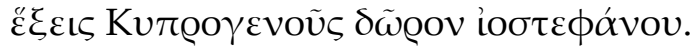

Das obelisierte Textsegment am Anfang des dritten Verses wird von Young gedruckt

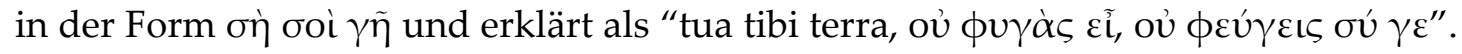
Selbst wenn die Worte das bedeuten können, fragt man sich, wie sich hieran où $\delta \dot{\varepsilon}$

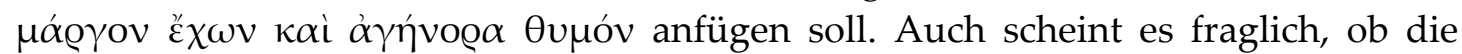

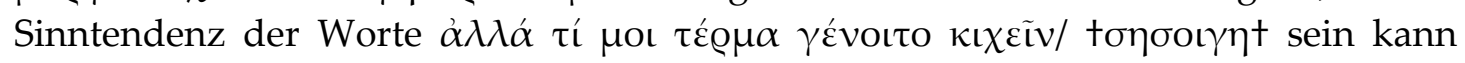
"May there come some end to my eager desire for you?" (Gerber), woran sich

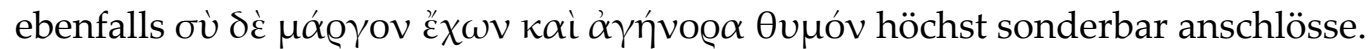

Dieser deutlich adversative Anschluß dürfte die schroffe Abneigung des puer dem Versuch seitens des dichterischen Ichs entgegensetzen, dessen Zuneigung zu

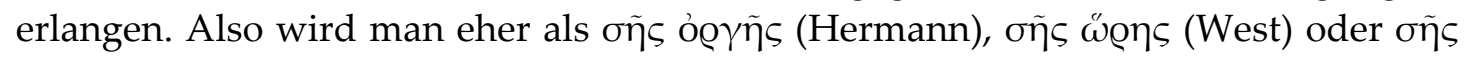

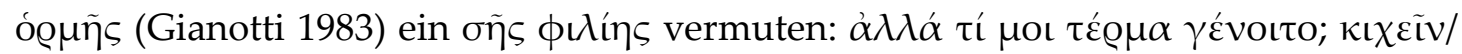

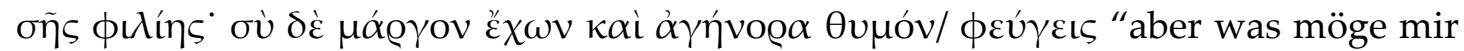
als Ziel (meiner Verfolgung) zuteilwerden? Deine Freundschaft zu erlangen. Du

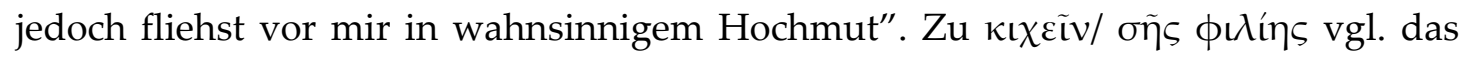

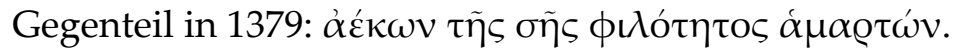

1300 f. wird in dieser Textfassung imitiert bei Meleager AP XII $158=$ HE 44964503:

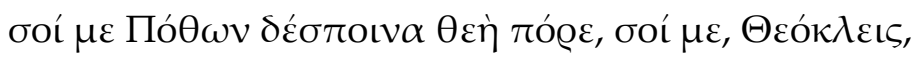
$\alpha \beta \varrho 0 \pi \varepsilon ́ \delta \iota l o s$ ”E

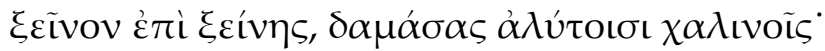

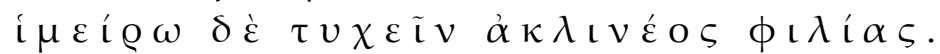

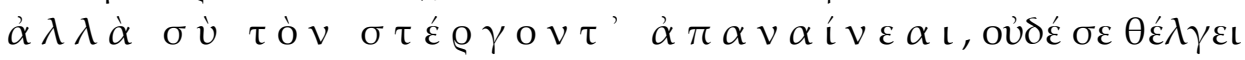

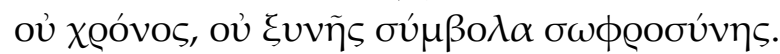

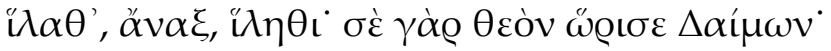

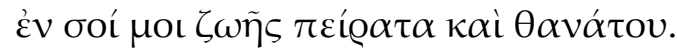

$1327-1334$

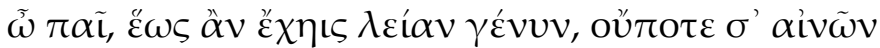

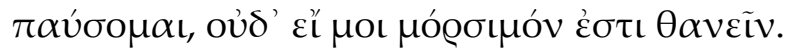

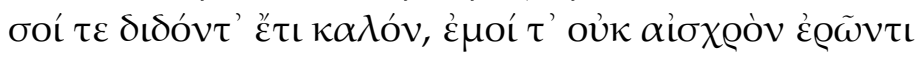

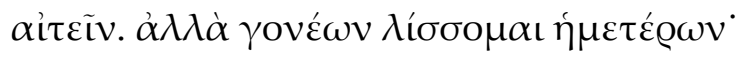

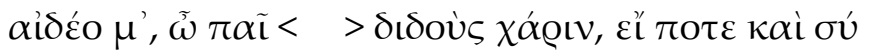

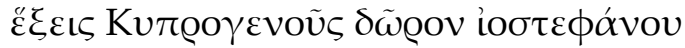




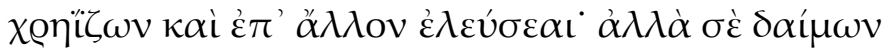

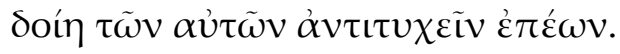

Wenn das Distichon $1327 \mathrm{f}$. mit dem Folgenden zusammengehört, ergibt sich ein gedanklicher Zusammenhang nur, wenn man für das überlieferte $\sigma \alpha i ́ v \omega v$ nicht $\sigma^{\prime}$

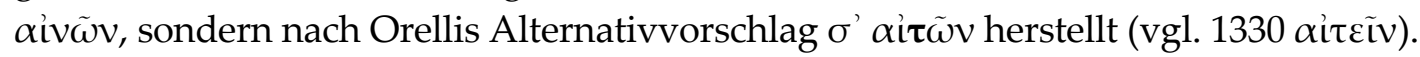

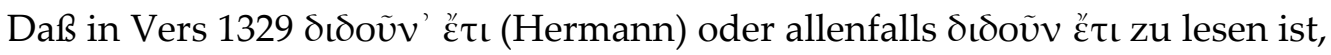
wird zu 1323-1326 gezeigt.

Die Lücke in Vers 1331 sähe man lieber als durch $<\tau \eta ́ v \delta \varepsilon>$ (van Herwerden,

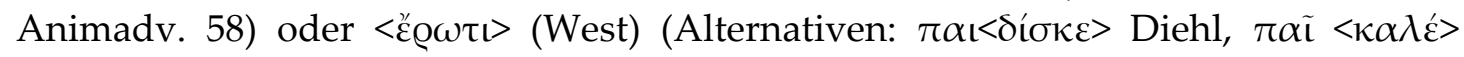
Bergk, $\pi \alpha \tilde{\mathrm{l}},<\pi \alpha \iota \delta i>$ Kalinka, $\pi \alpha \tilde{\mathrm{l}},<\dot{\varepsilon} \mu \mathrm{i} \delta \dot{\varepsilon}>\delta i ́ \delta o v$ Passow) durch ein das Partizip in

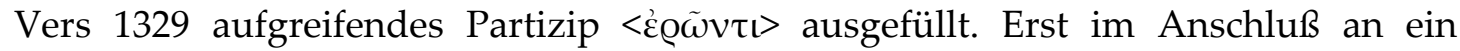
solches Partizip entfaltet der mit $\varepsilon$ ľ $\pi 0 \tau \varepsilon$ beginnende Bedingungssatz, der auf die

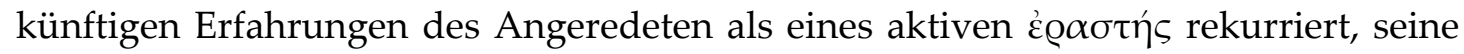
volle Wirkung.

In diesem Bedingungssatz kann $\varepsilon^{\natural} \xi \varepsilon\llcorner\varsigma$ trotz des wörtlich übereinstimmenden Pentameters 1304 (Hudson-Williams tilgt 1332 als interpolierten Lückenfüller) nicht richtig sein, da es hier nicht (wie dort) um den Vollbesitz der Gaben Aphrodites geht, sondern um eine zukünftige Situation intensiven erotischen Verlangens (komplizierte Versuche, im hier vorliegenden Kontext mit $\varepsilon^{\|} \xi \varepsilon\llcorner\varsigma$ fertigzuwerden, bietet

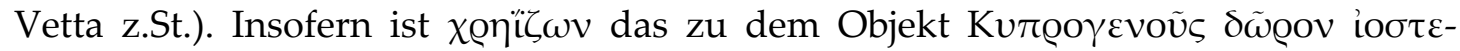

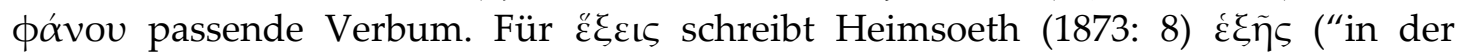
Folge", sonst in den Theognideen nicht belegt), aber man vermißt ein mit $\kappa \alpha \grave{\iota}$ '̇ं '

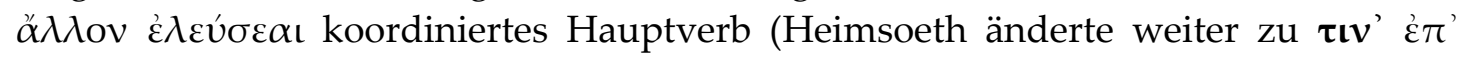

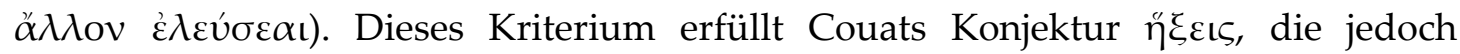
$\varepsilon \grave{\lambda} \varepsilon v ́ \sigma \varepsilon \alpha \iota$ unschön antizipiert. Wenn man die Herstellung eines erst in späterer Liebesdichtung belegten Verbums riskieren will, kann man eine passende Variation $\mathrm{zu}<\dot{\varepsilon} \varrho \tilde{\omega} \nu \tau 1>$ herstellen:

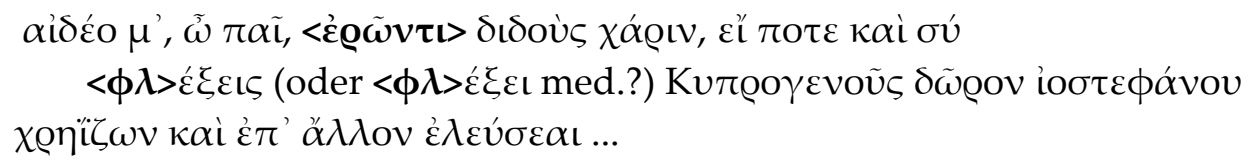

“Gewähre mir Liebendem deine Gunst, Knabe, wenn auch du einst aus Verlangen nach dem Geschenk der veilchenbekränzten Kypris lodern und dich zu einem anderen (Knaben) hinbegeben wirst ...". $\mathrm{Zu}$ intransitivem und metaphorischem $\phi \lambda \varepsilon ́ \gamma \varepsilon \iota v$ vgl. LSJ s.v. B 2.

Eine Imitation der Verse $1331 \mathrm{f}$. begegnet bei Straton Anth. Pal. XII 16, $3 \mathrm{f}$.

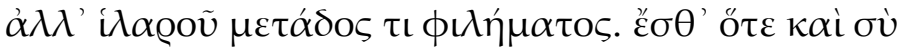

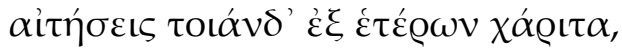

wo man das Asyndeton '̌́ $\sigma \theta^{\prime}$ ó $\tau \varepsilon$ und die fragwürdige Verwendung dieses Ausdrucks von einem einzigen ungewissen Zeitpunkt in der Zukunft (laut Kuehner/

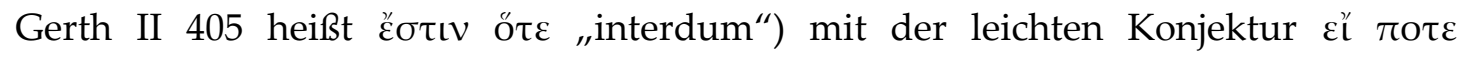
zugunsten einer dem Vorbild entsprechenden Beteuerung beseitigen könnte.

$901 \mathrm{f}$.

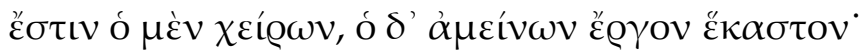

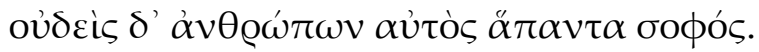




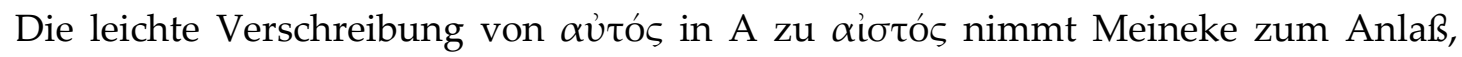

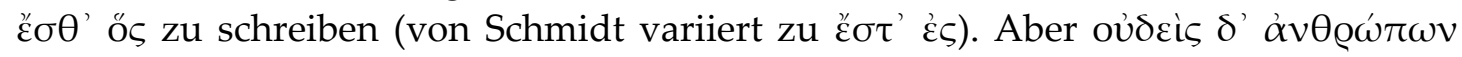

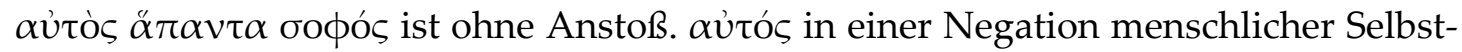
genügsamkeit wie an der gleichfalls umstrittenen Stelle Eur. Tro. 1203-1206:

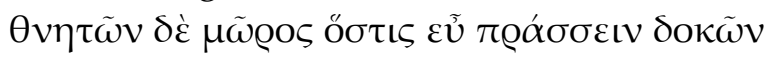

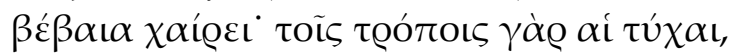

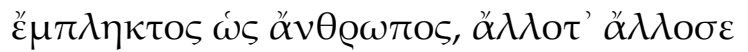

$\pi \eta \delta \tilde{\omega} \sigma \mathrm{l}, \kappa \circ \dot{v} \delta \varepsilon \dot{\iota} \varsigma \alpha \dot{v} \tau \dot{o} \varsigma \varepsilon \dot{v} \tau \dot{v} \chi \varepsilon \tilde{\imath} \pi \mathrm{o} \tau \varepsilon$.

$1271-1274$

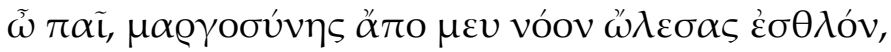

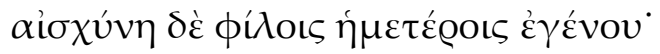

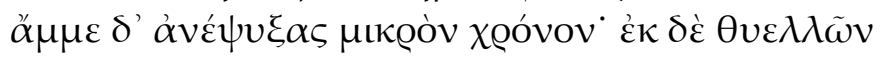

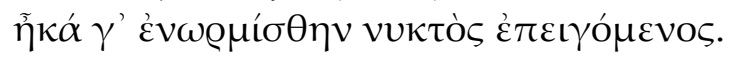

In Vers 1271 geht es um das Verlieren des eigenen Sinns (vgl. $35 \mathrm{f}$. ๆ̀

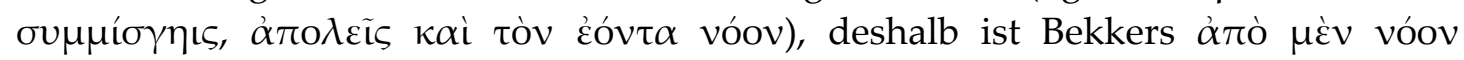

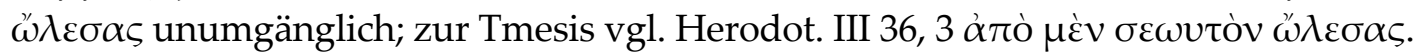

Die Heilung von Vers 1274 ergibt sich durch die Kombination der Vorschläge

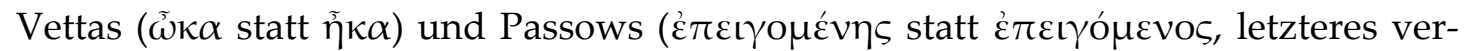

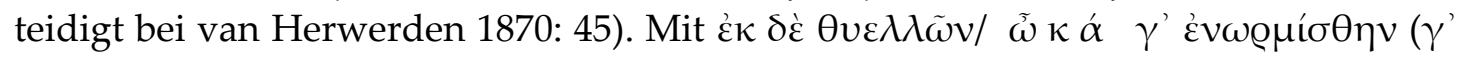

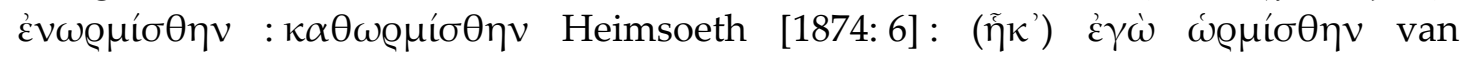

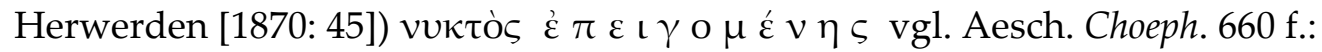

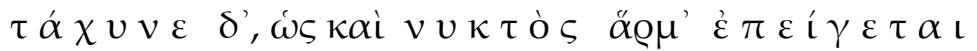

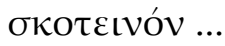

(10) Verunklarung anthetischer bzw. parallelistischer Redefiguren

Gelegentlich verlangen antithetische bzw. parallelistische Redefiguren in der überlieferten Fassung eine stilistische Schärfung.

$461 \mathrm{f}$.

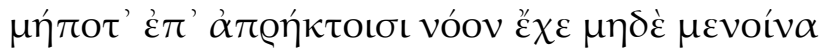

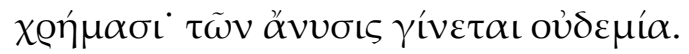

Die metrische Schwierigkeit im Hexameter wird man mit dem von Bergk erwogenen

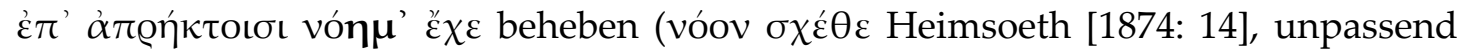

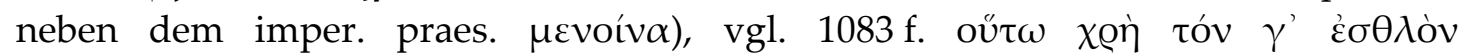

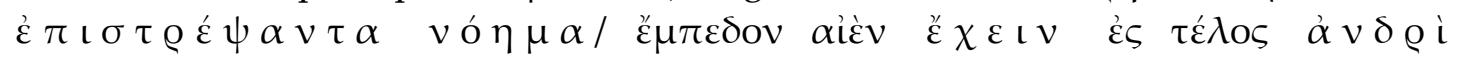

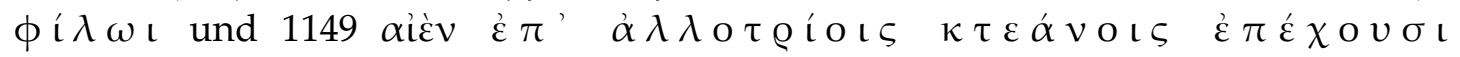
$v$ ó $\eta \mu \alpha$. Wesentlich weiter entfernt von der Überlieferung läge etwa ein $\varepsilon \dot{\pi}{ }^{\prime}$

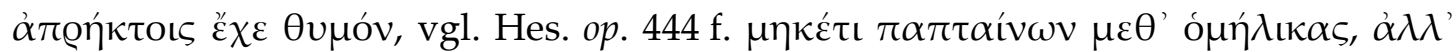
$\dot{\varepsilon} \pi \grave{i}$ है $\mathrm{\gamma} \omega / \theta v \mu$ òv है $\chi \omega v$. 


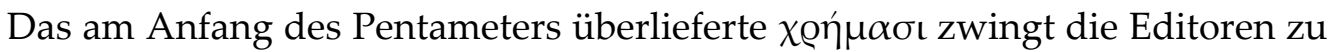

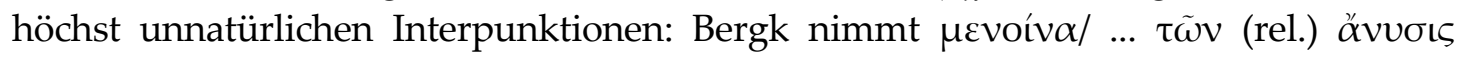

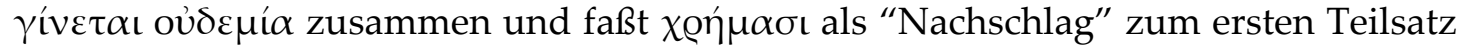

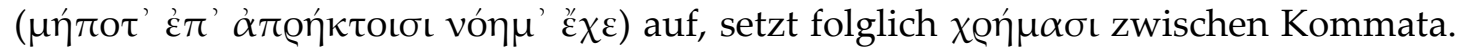
Im Apparat argumentiert er jedoch ausführlich, daß Belege für eine solch manirierte Wortstellung in der archaischen Dichtung fehlen, und erwägt, entweder $\mu \varepsilon v o i ́ v \alpha$ in

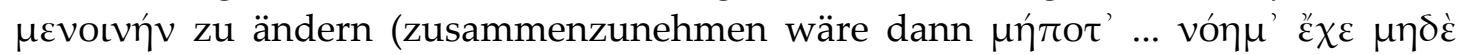

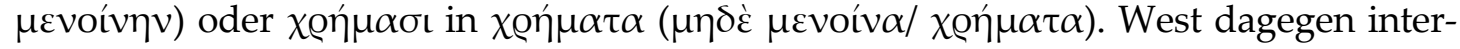
pungiert stark nach $\chi \varrho \eta \dot{\mu} \alpha \sigma \mathrm{l}$, hat dann im ersten Teilsatz immer noch eine wegen des nachklappenden $\chi \varrho \eta ́ \mu \alpha \sigma \iota$ ungewöhnliche Wortstellung und andererseits im Pentameter einen sachlich trivialen Schlußsatz ("das Unausführbare [ $\tau \tilde{\omega} v=\dot{\alpha} \pi \varrho \eta \kappa-$ $\tau \omega \nu]$ gestattet keine Vollendung"). Dagegen erhielte man einen formal vollendeten zweigliedrigen Parallelismus, wenn man $\tau \tilde{\omega} v$ weiterhin als Relativpronomen auffaßt

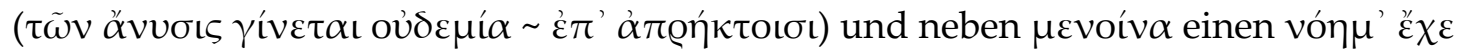
entsprechenden dativischen Ausdruck der psychischen Instanz herstellt:

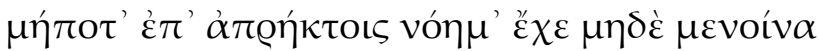

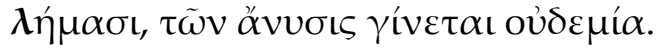

$547 \mathrm{f}$.

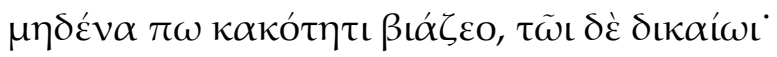

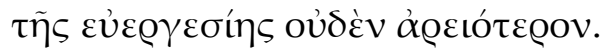

West hat die Worte $\tau \tilde{\omega} \iota \delta \dot{\varepsilon} \delta \iota \kappa \alpha i ́ \omega \iota$ zurecht syntaktisch vom Pentameter abgelöst. Als

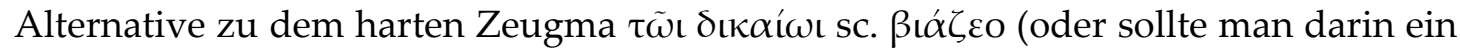
Oxymoron sehen, das freilich durch die Ellipse des Verbs einigermaßen verdunkelt würde?) böte sich ein durch eine leichte Textänderung erkaufter, formal perfekter

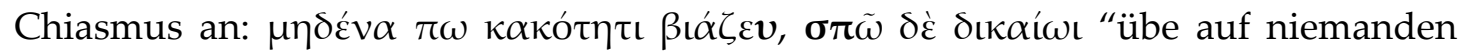
Zwang durch Schlechtigkeit aus, sondern ziehe ihn mit Gerechtigkeit auf deine

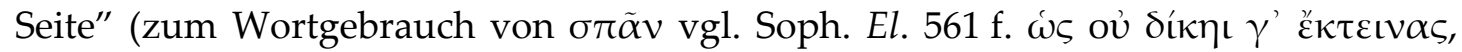

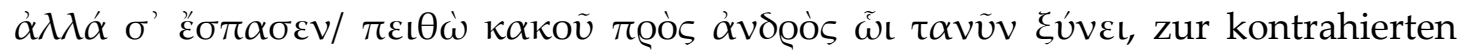
Form des medialen Imperativs auf $-\varepsilon v$ vgl. Theogn. 61. 220. 1050. 1073). Hieran

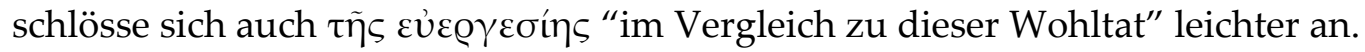

$731-736$

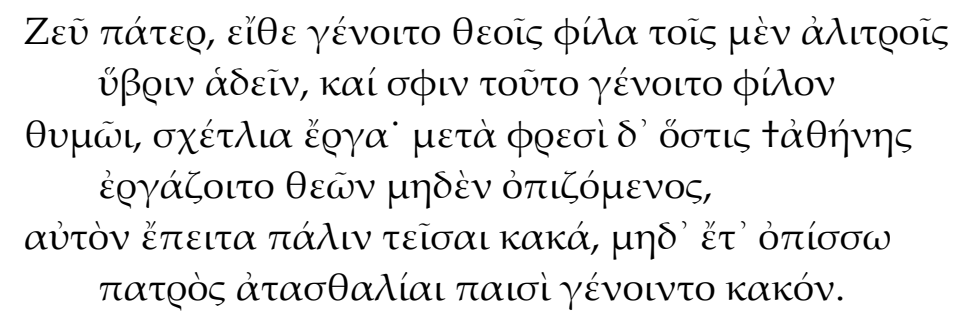

Das Gebet läuft offenbar darauf hinaus, daß die Bestrafung des Übeltäters zur Sühnung eines Verbrechens ausreicht und daß nicht auch noch dessen unschuldige Nachkommen in die Vergeltung miteinbezogen werden sollen. Der Gedanke scheint zunächst von $\varepsilon$ 


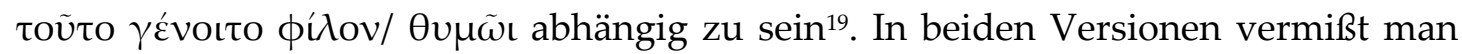
den Gedanken "zur Genüge büßen”. Der ursprünglichen Fassung nahekommen könnte folgendes:

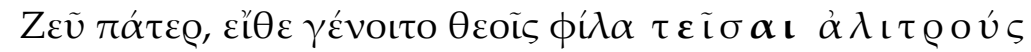

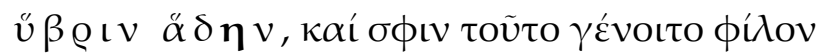

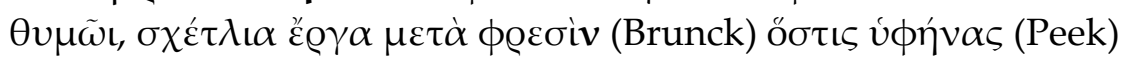

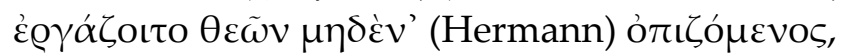

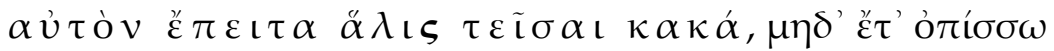

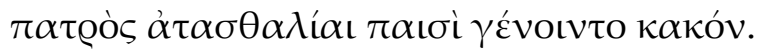

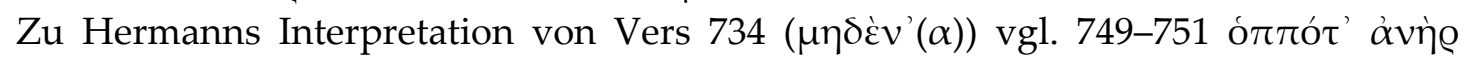

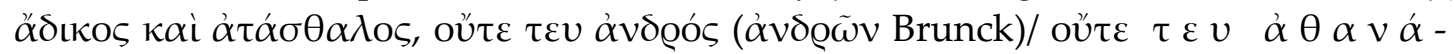

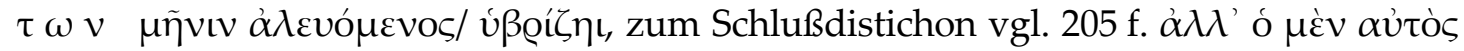

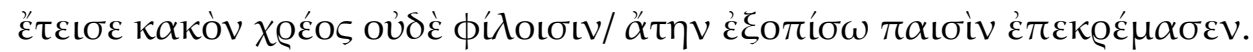

Die hiatverschließende Wirkung des Digamma in $\varepsilon^{\prime} \tau \varepsilon \iota \tau \alpha ~ \alpha ٌ \lambda ı \varsigma$ genau wie in

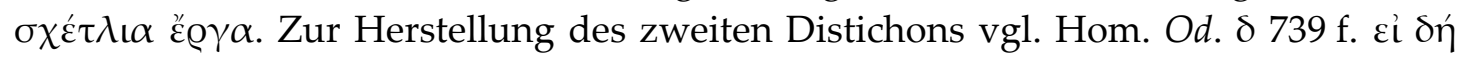

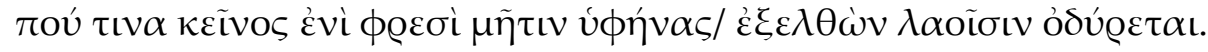

$823 \mathrm{f}$.

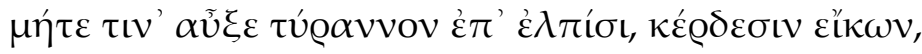

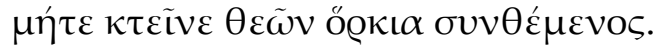

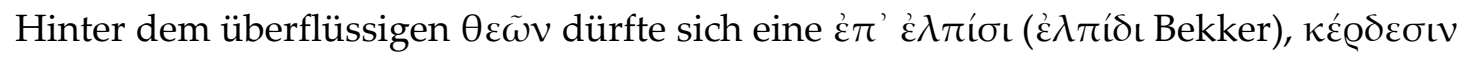
$\varepsilon$ عॉ $\omega \nu$ entsprechende, die Antithese formal abrundende Explikation der psychologi-

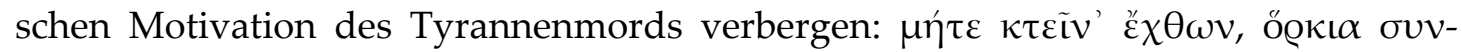

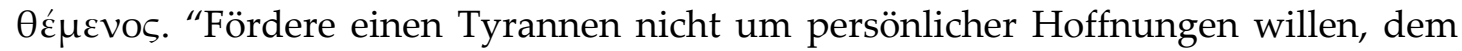
Gewinnstreben nachgebend, aber töte ihn auch nicht aus Haß, einen Eid gegen ihn

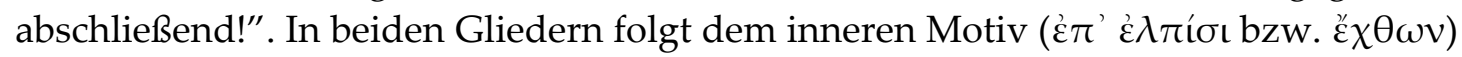

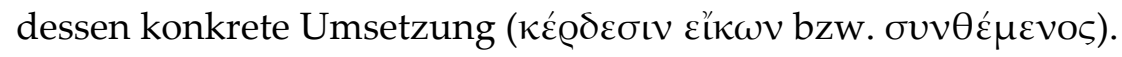

$947 \mathrm{f}$.

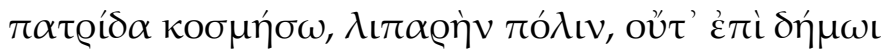

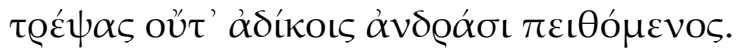

Die Tempusdifferenz zwischen den eng koordinierten Partizipien $€ \varrho \varepsilon ́ \psi \alpha \varsigma$ und

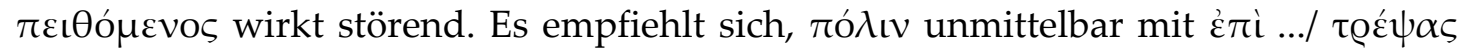

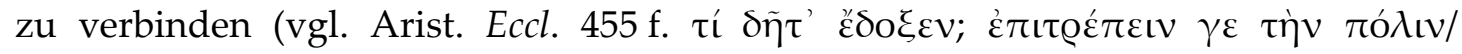

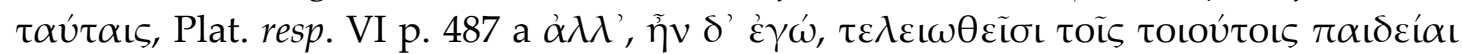

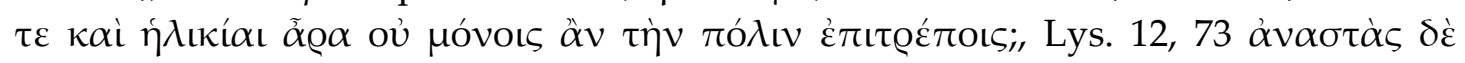

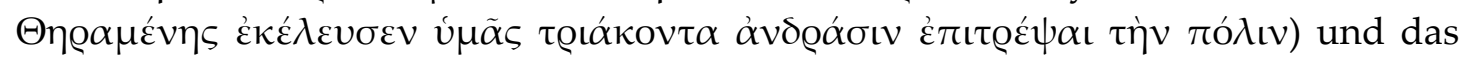
zweite Partizip so zu ändern, daß es auf beide Glieder bezogen werden kann:

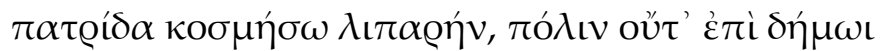

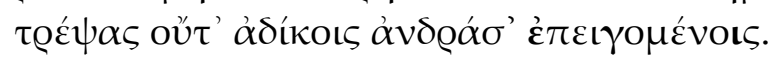

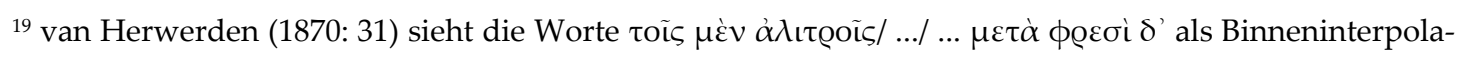
tion an. 


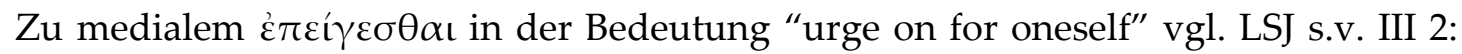
Solchem beiderseitigen Drängen gab der Sprecher (gemäß Bergk der Athener Solon) eben nicht nach. Die Elision der Endung - $\sigma \iota$ im Dativ Plural der konsonantischen Deklination ist (im Gegensatz zu derjenigen des singularischen -l, vgl. zu 1323-1326) gesichert durch das in seiner Authentizität unanfechtbare Siegelgedicht, 24-26 (diese kardinale Stelle fehlt bei West 1974: 116):

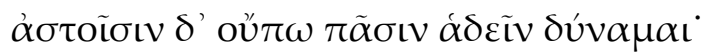

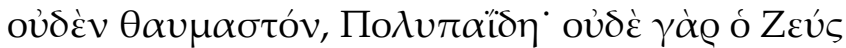

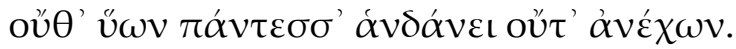

Ferner ist eine solche Elision sehr wahrscheinlich in $521 \mathrm{f}$.

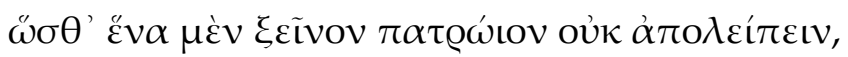

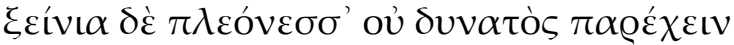

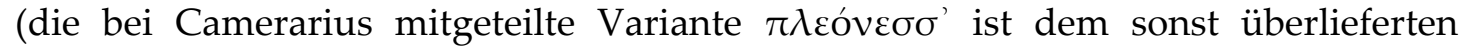

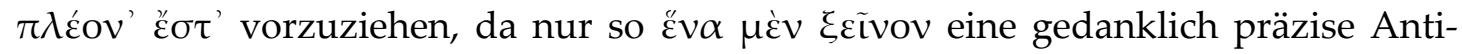
these erhält).

$1219 \mathrm{f}$.

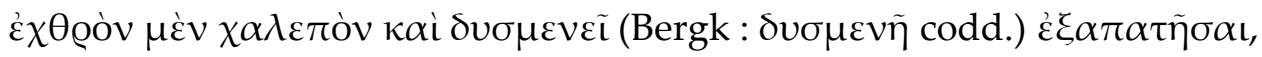

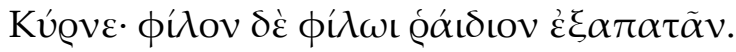

Die mit einer nicht gerade schönen Wiederholung verbundene unmotivierte Tempusdifferenz zwischen $\grave{\varepsilon} \xi \alpha \pi \alpha \tau \tilde{\eta} \sigma \alpha \iota$ und $\grave{\varepsilon} \xi \alpha \pi \alpha \tau \tilde{\alpha} \nu$ läßt sich beseitigen, indem man letzteres in das epische Verbum $\dot{\varepsilon} \xi \alpha \pi \alpha \phi \varepsilon \tilde{\imath} v$ ändert. Die Variation ist ihrerseits

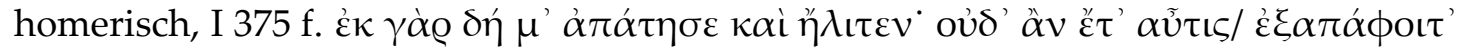

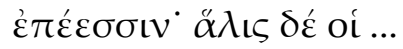

$1311-1318$

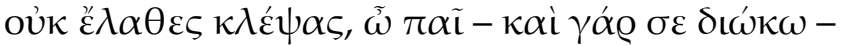

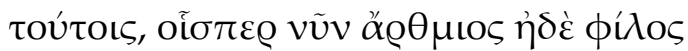

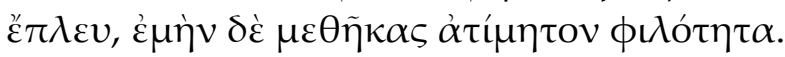

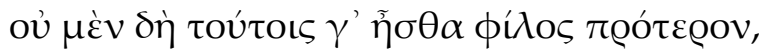

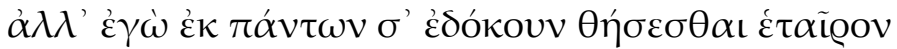

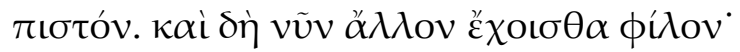

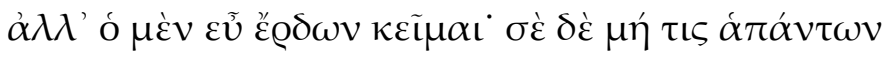

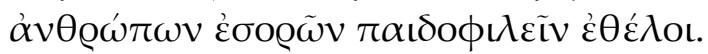

West (1974: 167) nimmt die Verse 1312 f., die früher als Determination eines epexegetischen toútoıs $\gamma^{\prime}$ (1314) verstanden wurden, mit dem Auftaktsatz zusammen.

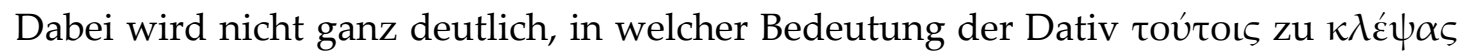
gezogen werden soll: etwa als dativus commodi "du betrogst mich zugunsten derer, ...$\left.^{\prime 20}\right)$ ?

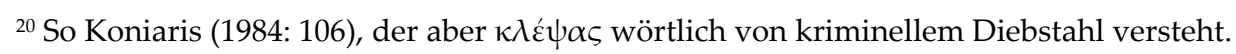


Gelöst werden die Probleme erst, wenn man die Verse 1312 f. als syntaktisch eigenständigen Satz nimmt. Hierzu ist nur die Abänderung eines Buchstabens erforderlich:

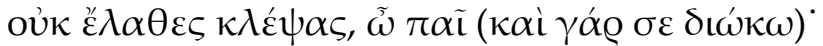

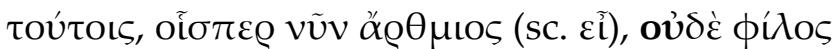

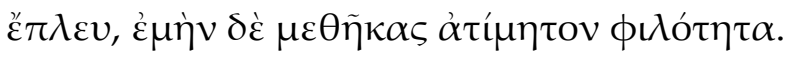

Dann wird das im Folgenden behandelte Thema (1314 ff.: Freundschaft mit zuvor Unbekannten, Verstoßung des vertrauten Freundes) bereits hier eingeleitet:

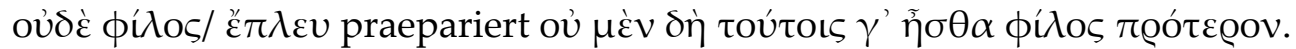

In Vers 1315 wurde $\theta \eta \dot{\sigma \varepsilon \sigma \theta \alpha \iota ~ v o n ~ S e i d l e r ~ a u s ~ u ̈ b e r l i e f e r t e m ~} \sigma \eta ́ \sigma \varepsilon \sigma \theta \alpha \iota$ hergestellt. Als Alternative wäre ein die Besitzansprüche des Sprechers deutlicher

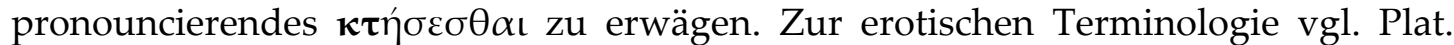

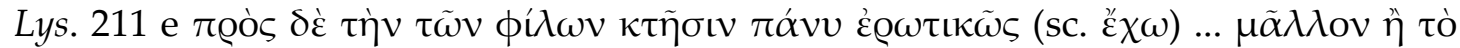

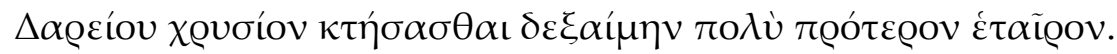

(11) Verlust der sprachlichen Praegnanz

Mitunter scheint die Praegnanz der Sprache in der Überlieferung gelitten zu haben.

$971 \mathrm{f}$.

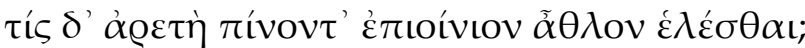

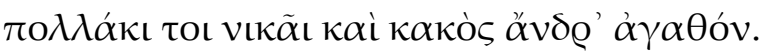

In der sentenziösen Zuspitzung im Pentameter vermißt man die Beschränkung aufs Trinken, die das Ganze erst zu einer praegnanten Sentenz macht. Da adverbiales

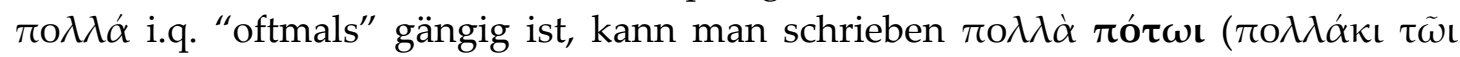

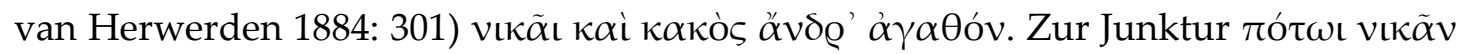

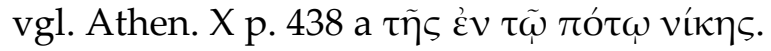

$1185 \mathrm{f}$.

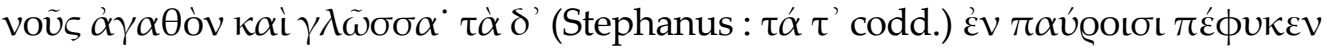

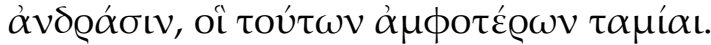

Der entscheidende Gedanke des Zusammentreffens von voũ mit Änderung eines einzigen Buchstaben sehr deutlich ins Licht rücken²1:

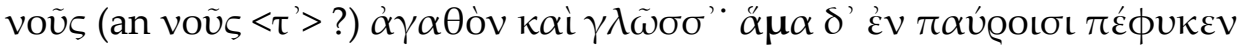

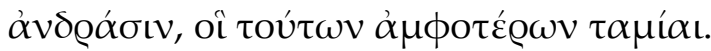

$1191 \mathrm{f}$.

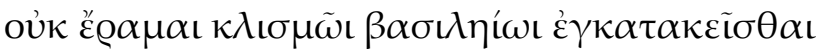

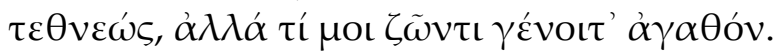

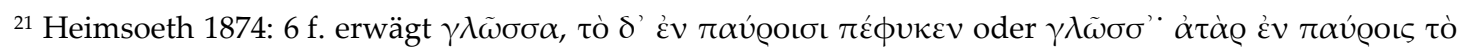
$\pi \varepsilon \dot{\phi} \cup \kappa \varepsilon v$.
} 


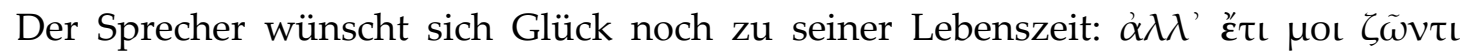

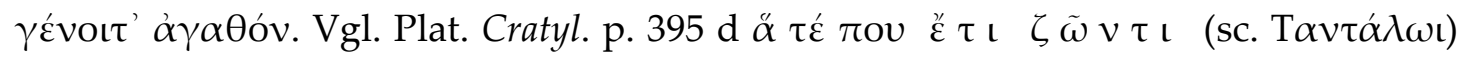

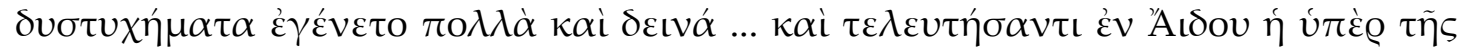

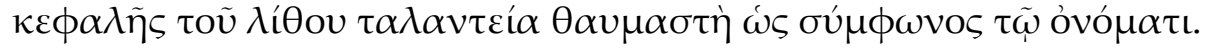

$1239 \mathrm{f}$.

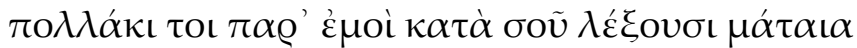

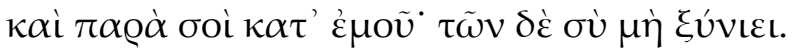

$\mathrm{Zu} \tau \tilde{\omega} v \delta \varepsilon$ würde es gut passen, wenn die Identität der Lästerer vorher spezifiziert

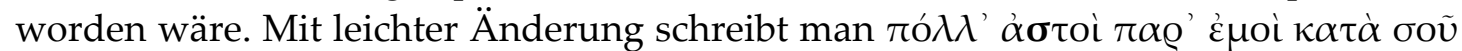

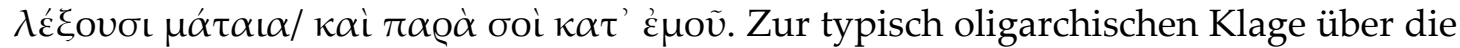

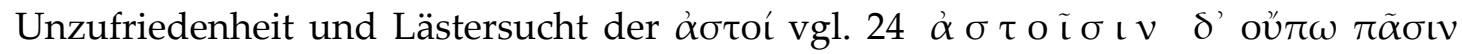

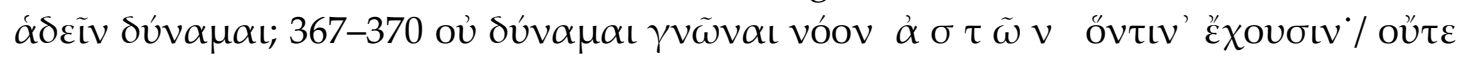

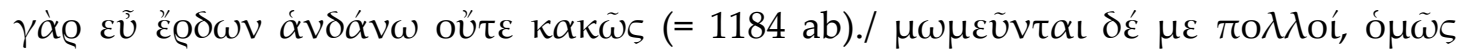

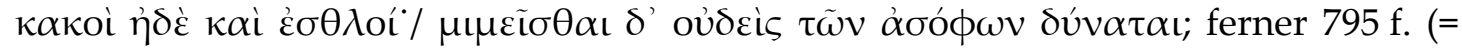

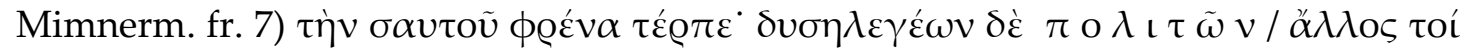

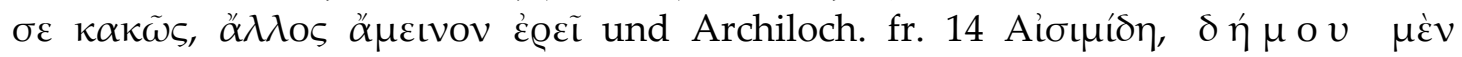

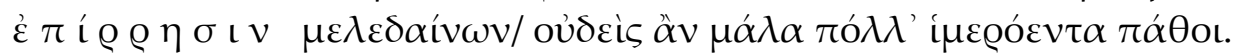

Auf dem Wege solcher Korruptelen kann die Schlußpointe eines epigrammartigen Gebildes unkenntlich werden:

959-962

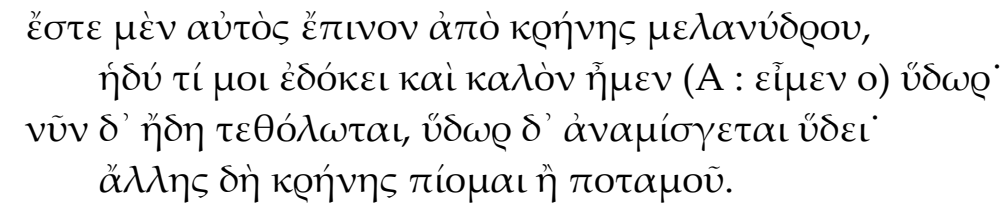

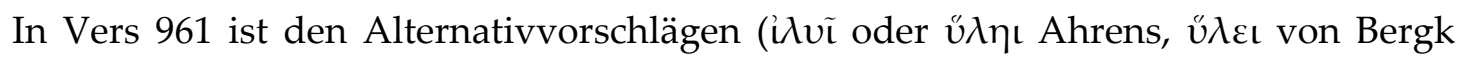
erwogen) die Konjektur $<0>v \ddot{\delta} \varepsilon \iota$ vorzuziehen (Allen 1933: 53). Abgesehen von der

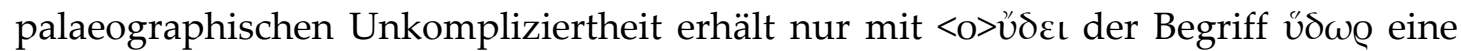
derart grundsätzliche Antithese, daß es gerechtfertigt erscheint, an einen Satz, in

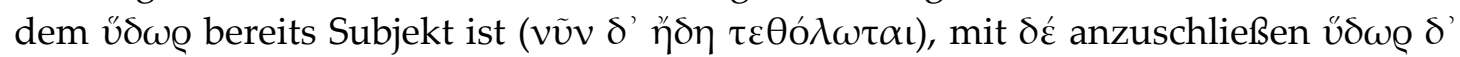

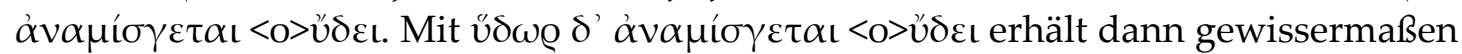
der Begriff $\tau \varepsilon \theta$ ó $\lambda \omega \tau \alpha \iota$ eine epexegetische Erklärung. Trotzdem stößt sich West (1974: 161) nicht zu Unrecht an dem gleichbleibenden Subjekt und erwägt daher ăoıs $\delta$ '

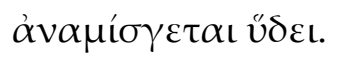

Das Problem des gleichbleibenden Subjekts läßt sich mit einem weiterem Eingriff beheben, der jedoch am vorigen Satz anzusetzen hat und zugleich den

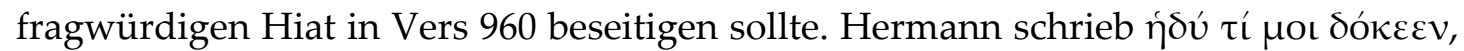

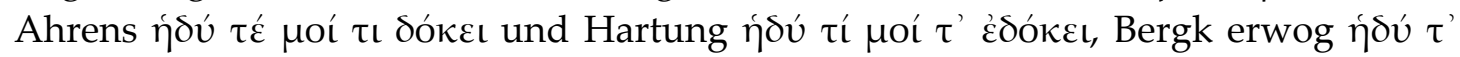

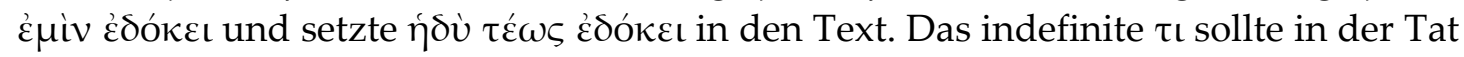
verschwinden, aber die Form $\varepsilon \dot{\mu} \mu$ ív ist in der Elegie nicht belegt (West 1974: 100), und eliminieren darf man das Personalpronomen auch nicht, da es gerade auf die 
subjektiven Erfahrungen ankommt, die der Sprecher gemacht hat. Diesen Aspekt kann man folgendermaßen idiomatisch verstärken:

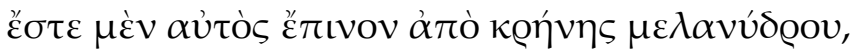

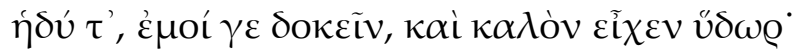

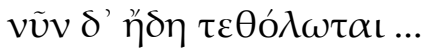

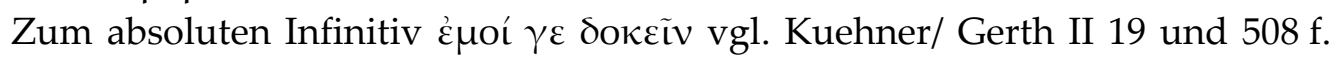

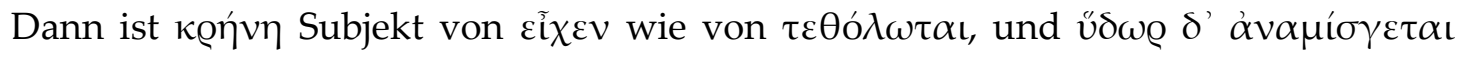

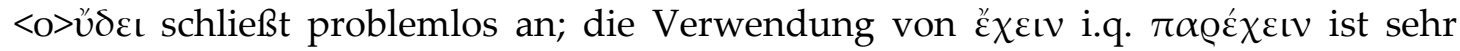

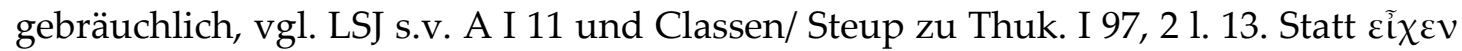
könnte man auch einen dichterischeren Ausdruck herstellen, etwa $\kappa \alpha i ̀ ~ \kappa \alpha \lambda$ òv í $\varepsilon$

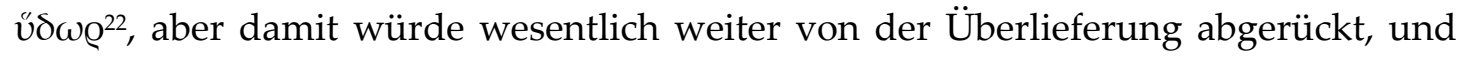
aus der Perspektive des "Trinkenden" paßt "die Quelle bot Wasser" besser als ein pittoreskes "die Quelle verströmte Wasser".

Als weitere Schwierigkeit verbleibt die Deutung des Schlußpentameters. Gomme (1925: 101) und Allen a.a.O. wenden sich zurecht gegen die Deutung von

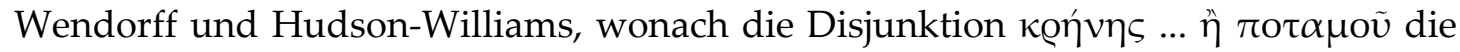
Alternative zwischen weiblicher Geliebter und männlichem Geliebten bezeichnen

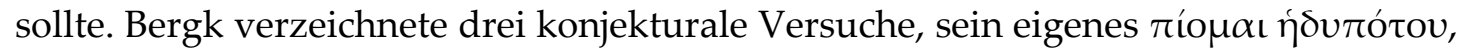

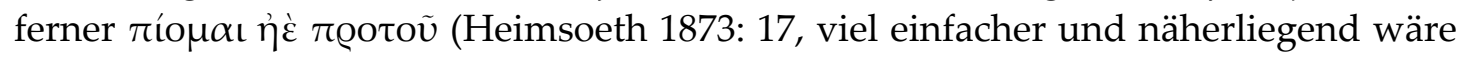

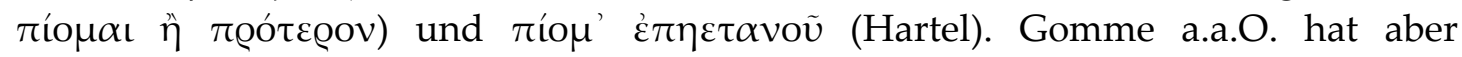

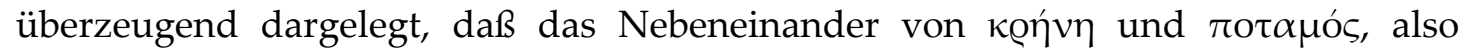
(reiner) Quelle und (schlammigem) Fluß, für einen Griechen durchaus Sinn ergibt.

Nach Gomme hätte der Schlußsatz des (erotischen) Gedichts die Bedeutung "I will find me another mistress or pick up a girl from anywhere, the market-place or a brodel", ähnlich Allen a.a.O.: "Theognis says he might take to what we might call the drain, sink, street" (die im folgenden angeführten Parallelen machen deutlich, daß auch er an Prostitution denkt). Aber kann der Dichter, wenn er in den ersten drei Versen so nachdrücklich den Unterschied zwischen der früheren quellenhaften Reinheit (1 f.) seines Geliebten (die Umgebung der Theognideen läßt eher an ein homoerotisches Verhältnis denken) und der späteren flußhaften Verschmutzung (3) herausgestellt hat, jetzt so einfach sich der Disjunktion bedienen "dann werde ich eben aus einer anderen Quelle oder einem (anderen) Fluß trinken", als würde dies gar keinen Unterschied ergeben, ob er einen "reinen" Geliebten (entsprechend dem früheren Zustand des ehemaligen) oder einen "verschmutzten" (entsprechend dessen jetzigem Zustand) findet? Die Aussage des Gedichts wäre dann etwa "Früher, als ich mit dir verkehrte, warst du rein, jetzt bist du verschmutzt; also suche ich mir einen anderen Reinen oder Verschmutzten".

Die Schlußfolgerung, die das dichterische Ich nach der gemachten Erfahrung der Depravation des Geliebten zieht, kann doch nur diejenige sein, daß er sich einen anderen suchen wird, der so rein ist, wie es der Ehemalige früher war, da er dessen jetzigen Zustand nicht mehr ertragen kann. Gomme hat die erforderliche Konjektur mit seiner Formulierung "men drank water drawn from a spring, no t f r o m a

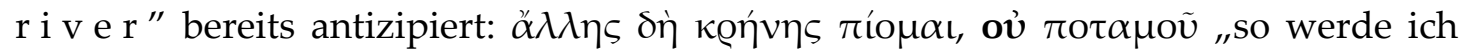
eben aus einer anderen Quelle trinken, nicht aus dem Fluß (der du jetzt bist)“. Im

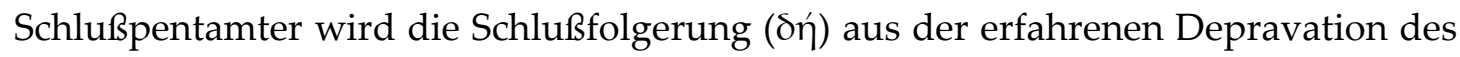

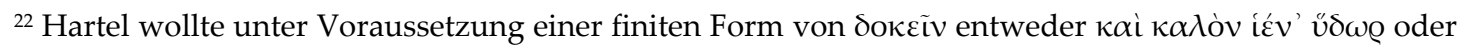

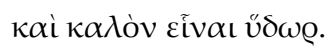


ehemaligen Geliebten gezogen: Das dichterische Ich will sich einen anderen quellenhaft reinen Geliebten suchen und nicht mehr aus dem (schlammig gewordenen) Fluß trinken. In der Abschiedserklärung im Schlußpentameter wird die Antithese sprachlich so schroff ausgedrückt wie nur möglich, nämlich mit einem zwei konträre Substantive kontrastierenden ovं; zu dieser "nachdrücklicheren" Alternativkonstruktion zu oủঠé bieten Kuehner/ Gerth II 293 einige dichterische Parallelen.

Im ganzen:

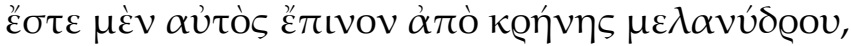

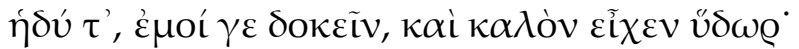

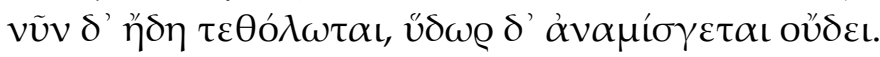

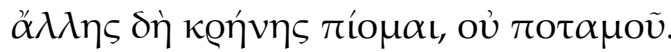

\section{(12) metrische Trugschlüsse}

Auch metrische Trugschlüsse können durch die korrumpierende Wirkung der Überlieferung zustandekommen.

$1323-1326$

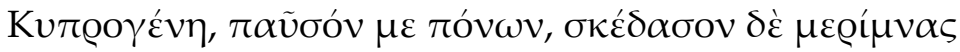

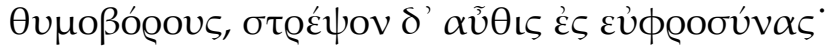

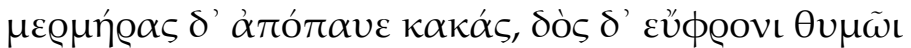

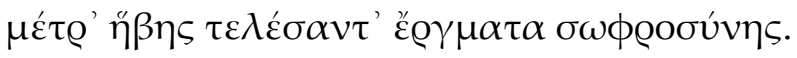

Die Elision der singularischen Dativendung -l, die der archaischen Elegie sonst fremd ist, wird von West (1974: 116) durch zwei Parallelen aus den Theognideen belegt:

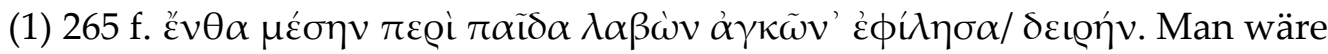
froh, wenn sich die exegetischen Schwierigkeiten des Gedichts (vgl. West 1974: 152) so leicht beheben ließe wie die sprachliche Anomalie durch Hermanns Konjektur

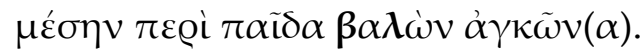

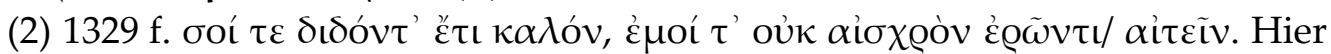

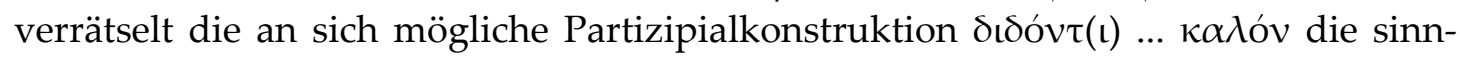

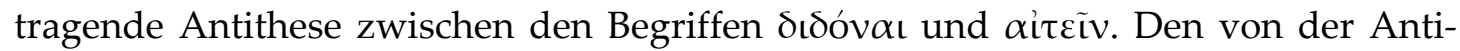
these schreiend eingeforderten Infinitiv erhält man relativ leicht mit Hermanns

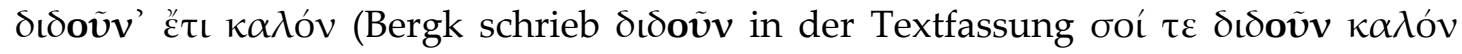

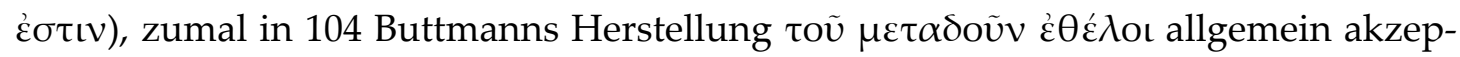
tiert ist (West erwägt $\mu \varepsilon \tau \alpha \delta o \tilde{v} v^{\prime} \varepsilon \dot{\varepsilon} \theta \dot{\lambda} \lambda \mathrm{ol}^{23}$ ). Übrigens findet sich bei dem hellenisti-

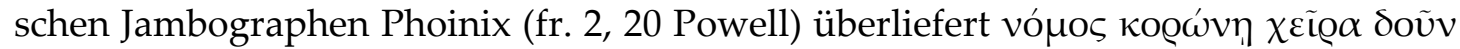

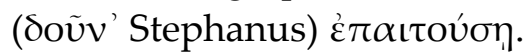

Demnach spricht in dem hier besprochenen Gedicht prima facie nur sehr wenig für ein $\tau \varepsilon \lambda \varepsilon ́ \sigma \alpha \nu \tau(\iota)$. Aber auch hier geben inhaltliche Argumente den Ausschlag zur konjekturalen Abänderung: Ein vom Liebeskummer gequälter Mensch

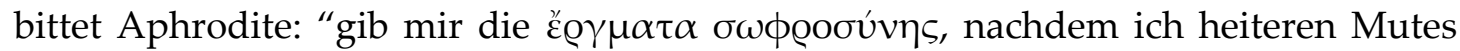
das Vollmaß der Jugend vollendet habe". Zunächst einmal ist Aphrodite für die ع̌@ $\gamma \mu \alpha \tau \alpha \sigma \omega \phi \varrho o \sigma u ́ v \eta \varsigma$ einfach nicht zuständig. Man müßte das Schwergewicht ganz

${ }^{23}$ Allgemein zugunsten der elidierten Form auf - $v \alpha \iota$ in vergleichbaren Fällen van Herwerden 1870: 5. 
auf das Partizipialglied legen: “Laß mich das Vollmaß meiner Jugend heiteren Mutes

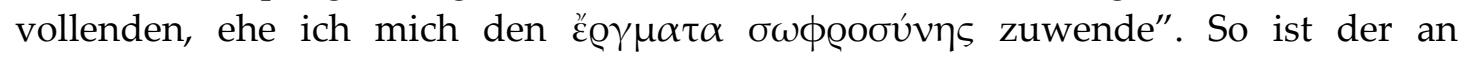

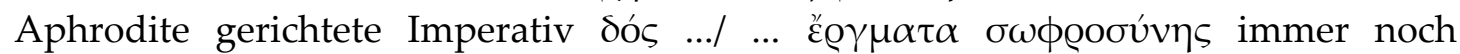
unbehaglich genug, aber vor allem ist es psychologisch höchst unplausibel, daß ein vom Liebeskummer geplagter Mensch seine jetzige Lebensphase nur als eine Vorstufe zu den ع̣̌ $\gamma \mu \alpha \tau \alpha \sigma \omega \phi \varrho o \sigma u ́ v \eta \varsigma$ ansieht, denen er sich anschließend zuwenden will. Formal müßte sich der Dichter ankreiden lassen, das ganz auf Heiterkeit und Sorgenfreiheit abgezweckte Gedicht in seinem Schlußhemistich mit dem gegen-

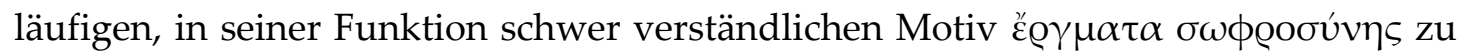
Ende gebracht zu haben.

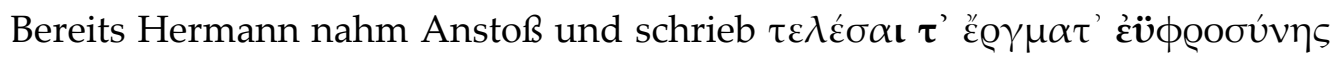

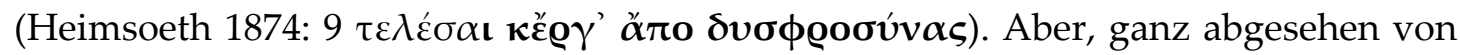
der irregulären Stellung des $\tau \varepsilon$, geht es nicht an, den nunmehr dritten Vers in Folge

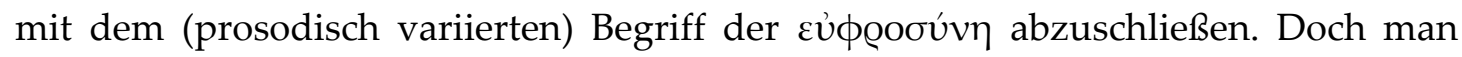
erwartet in der Tat eine (wahrscheinlich stilistisch anspruchsvollere) Variation von

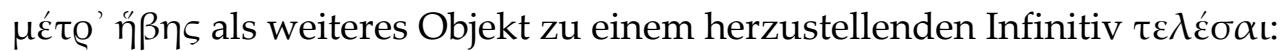

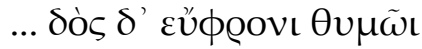

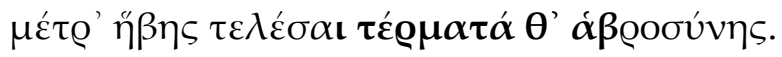

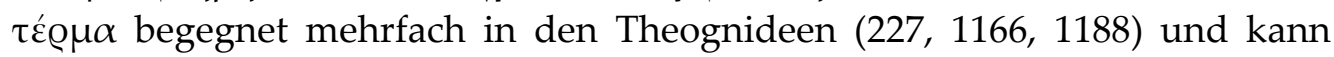
durchaus die erforderliche Bedeutung "culmination, highest point, goal" haben (LSJ s.v. II 3). $\alpha \beta$ Qoov́v $\eta$ bezeichnet die Verlockungen der Liebe in vergleichsweise gewählter Weise und wird durch die in anderem Zusammenhang stehende Stelle Xenophanes fr. eleg. 3, 1 West zugleich als elegisch erwiesen.

$831 \mathrm{f}$.

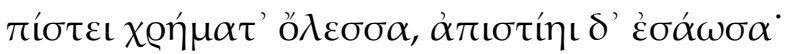

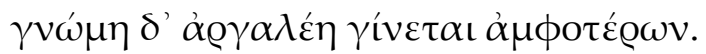

West erwägt eine Änderung in $\chi \varrho \tilde{\eta} \mu \alpha \dot{\alpha} \kappa$ 'ó $\lambda \varepsilon \sigma \sigma \alpha$, aber der Singular $\chi \varrho \tilde{\eta} \mu \alpha$ erhält durch 197 nur eine fragwürdige Stütze, wo das Wort wahrscheinlich einfach "Ding" und nicht "Geld; Vermögen" bedeutet. Die Einfügung einer Modalpartikel freilich ist in der Tat verlockend, da man sein Vermögen nicht gleichzeitig verlieren und retten kann. Man sollte sie aber nur auf das erste Glied beziehen (anders West 1974: 159)

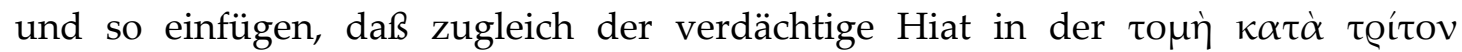

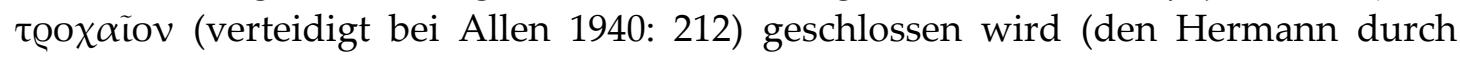
Abänderung von ǒ $\lambda \varepsilon \sigma \sigma \alpha$ in $\alpha \dot{\alpha} \omega \dot{\lambda} \lambda \varepsilon \sigma^{\prime}$ beseitigen wollte): $\pi i ́ \sigma \tau \varepsilon \iota ~ \chi \varrho \eta ́ \mu \alpha \tau$ ' ó $\lambda \varepsilon \sigma \sigma \sigma^{\prime}$

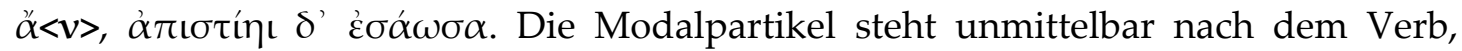

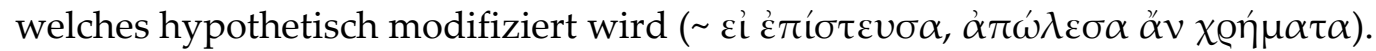

Der Pentameter bedeutet eher "beide Vorgänge lassen sich nur schwer begreifen sc. für den moralisch empfindenden Menschen" (Ahrens denkt wohl an zwei vom Sprecher faktisch erfahrene Tatsachen und ändert $\gamma \nu \omega \mu \eta$ in $\mu \nu \eta \dot{\mu \eta})$ als "both are difficult attitudes to adopt" (West 1974: 159). Zur Verbindung von $\gamma \nu \omega ́ \mu \eta$

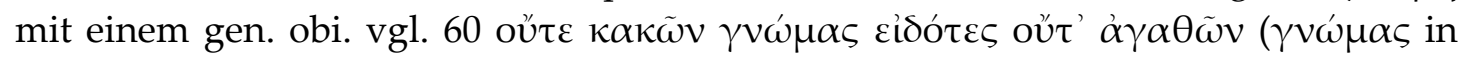
der Parallelüberlieferung $1114 \mathrm{zu} \mu \nu \eta \dot{\mu \eta} v$ trivialisiert). Wieder anders versteht van Herwerden (1870: 34) $\gamma \nu \omega \mu \eta$ als "sententia, quam is pronuntiat, qui iudex sedeat inter fidem et perfidiam". 


\section{(13) Glosseme}

Seltener lassen sich Unklarheiten der Überlieferung durch die Annahme eines Glossems erklären.

$261 \mathrm{f}$.

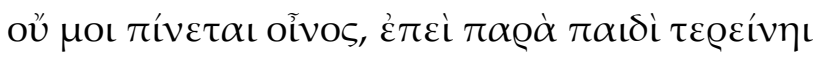

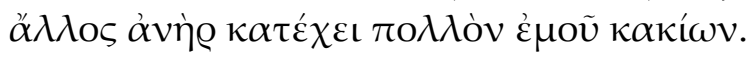

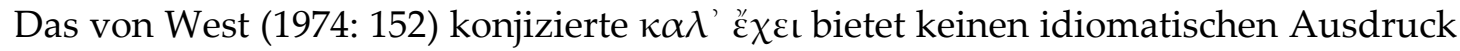
für die erotische Überlegenheit eines Widersachers. Hertzberg und Emperius

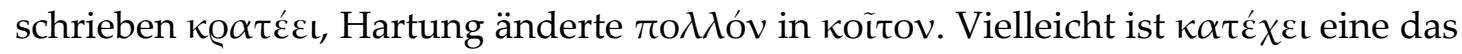
Versmaß restituierende Korruptel von unmetrischem $\kappa \alpha \theta \varepsilon v ́ \delta \varepsilon l$, was wiederum ein

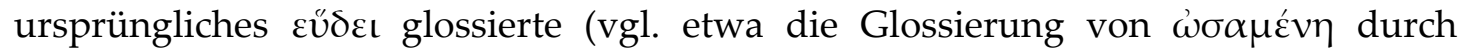

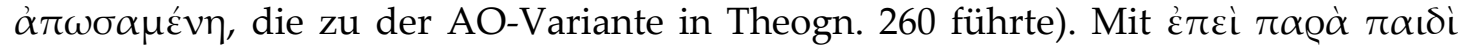

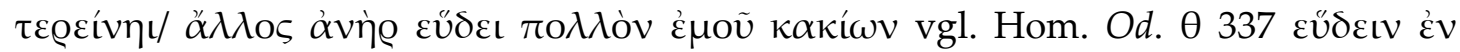

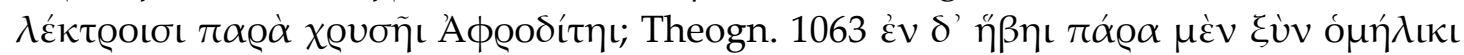

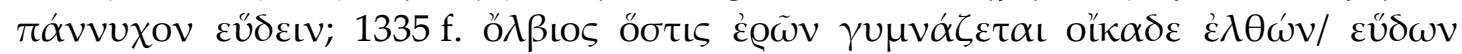

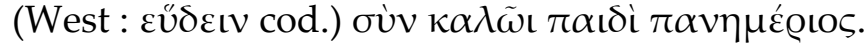

\section{(14) sonstige textkritische Probleme}

Es folgt eine Reihe nicht exakt zu klassifizierender textkritischer Probleme.

$69-72$

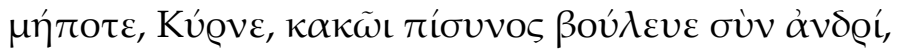

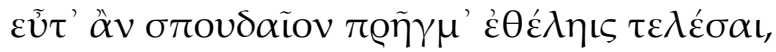

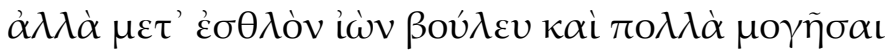

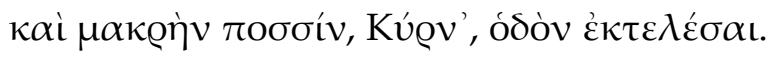

Im zweiten Distichon wird der Begriff des "Beratens" vermißt, der nur in dem Gedanken der Konsultation ( $\mu \varepsilon \tau^{\prime} \dot{\varepsilon} \sigma \theta \lambda$ òv $\left.i \omega \omega v\right)$ impliziert scheint. Die Varianten zur oben wiedergegebenen A-Überlieferung verraten das Bemühen, den vermißten

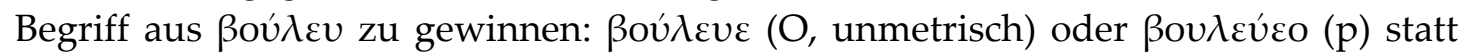

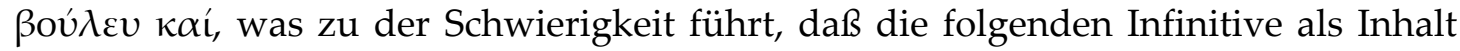
des Beratens gefaßt werden müssen; dies gibt wiederum Anlaß zur Herstellung der

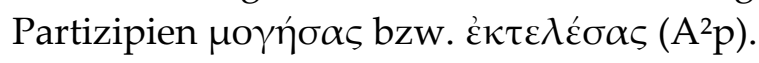

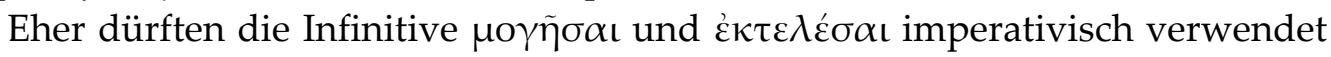
sein ${ }^{24}$; dann verbirgt sich das Ziel der vielen Mühen in dem leicht herstellbaren

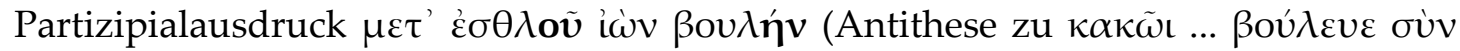
àvঠ@í): "Wenn du nach dem Rat eines guten Mannes gehst, nimm jedwede Mühe und jeden noch so weiten Weg auf dich".

${ }^{24}$ Zum Aufgreifen echter Imperative durch imperativische Infinitive vgl. Chantraine II 316. 


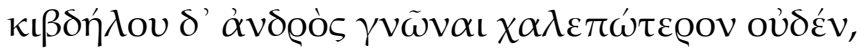

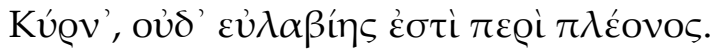

Für die Deutung "nor is there anything of greater value than caution" (HudsonWilliams, ähnlich van Herwerden 1870: 7: "nec quicquam est cautela praestantius") fehlt eben das Subjekt "anything" im Griechischen, und selbst wenn sie möglich

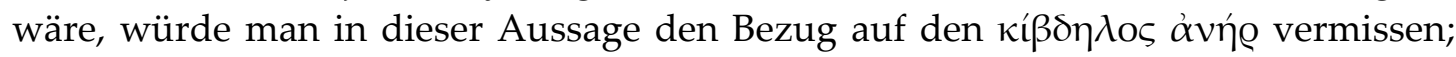
Gerber (1970: 281) ergänzt sehr hart aus dem Hexameter zu củ $\alpha \beta \beta$ ín als Genitivus

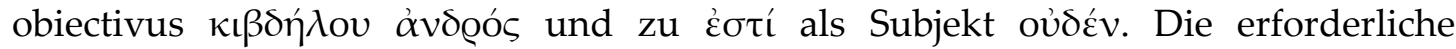
Aussage "nothing requires more caution than such a man" (Hudson-Williams) hat

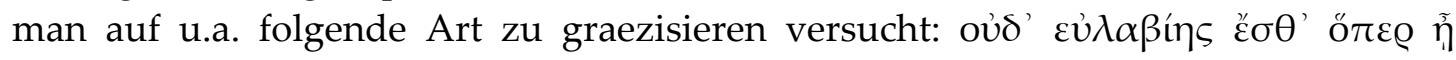

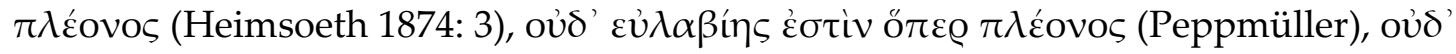

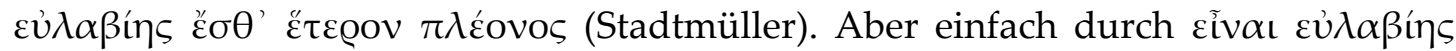
läßt sich der Gedanke "Vorsicht erfordern" kaum ausdrücken (trotz Euen. fr. 1, 5 f.

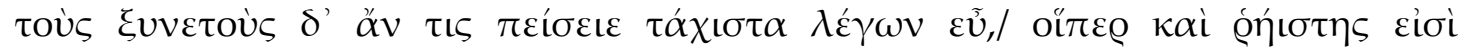

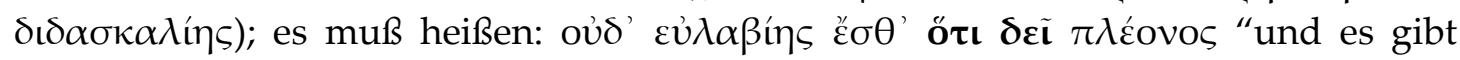
nichts, was größere Vorsicht erfordert" (M. Schmidt wollte oủ ' $\varepsilon \dot{u} \lambda \alpha \beta i ́ n \varsigma ~ \delta \varepsilon \tilde{\imath} \pi \varepsilon Q i ́$ $\tau \varepsilon v \pi \lambda \dot{\varepsilon}$ ovo $)$. Aktives $\delta \varepsilon \tilde{\imath} v$ kann gelegentlich mit einem nominalen Subjekt ver-

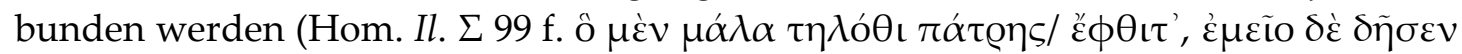

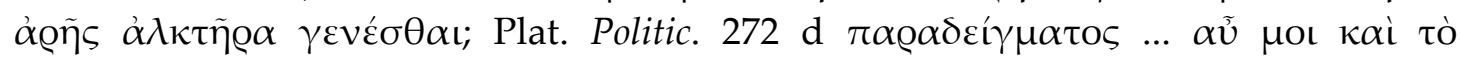

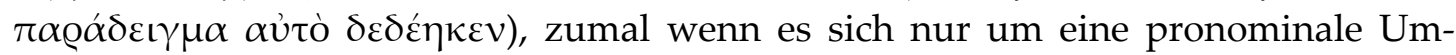

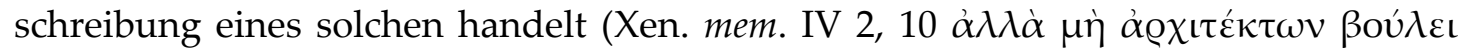

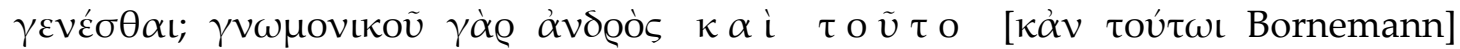

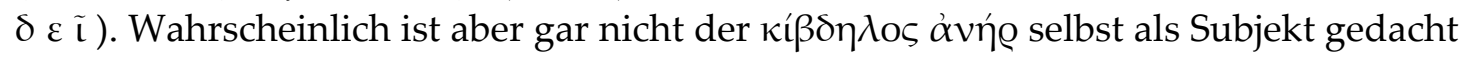
(in welchem Fall $\delta \varepsilon \tilde{\imath}$ heißen müßte "erfordert zwecks seiner Bekämpfung"), sondern

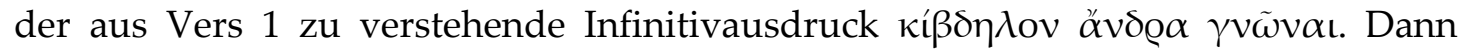
wäre gesagt, daß es höchste Vorsicht erfordert, einen solchen falschen Menschen zu entlarven (ohne dabei seinerseits zu Schaden zu kommen).

$155-158$

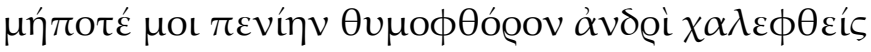

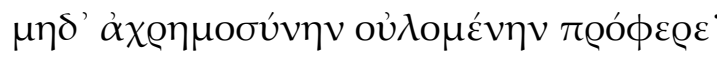

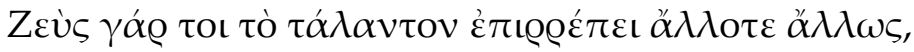

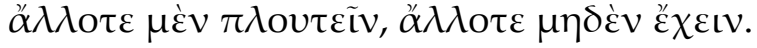

Der in der direkten Theognis-Überlieferung am Ende von Vers 157 überlieferte Dativ $\alpha ̋ \lambda \lambda \omega \iota$ paßt nicht in den Zusammenhang, da es nicht darum geht, daß bald der eine, bald der andere reich ist (vgl. Hudson-Williams z.St.: "Zeus shifts the balance for one man now, for another at another time ..., now for wealth, now for poverty"), sondern darum, daß Zeus die Schicksalswage für denselben Menschen bald hierhin, bald dorthin ausschlagen läßt. Dieser Gedanke wird freilich nicht durch das von West praeferierte (1974: 151), bei Stobaeus und Basilius überlieferte $\alpha ̋ \lambda \lambda \omega \varsigma$ ("er läßt die Schicksalswage bald auf diese, bald auf jene Weise ausschlagen") richtig zum 
Ausdruck gebracht, sondern nur durch ein aus $\alpha \ddot{\alpha} \lambda \lambda \omega \iota$ herzustellendes $\alpha \ddot{\alpha} \lambda \lambda \eta \mathbf{l}$, vgl.

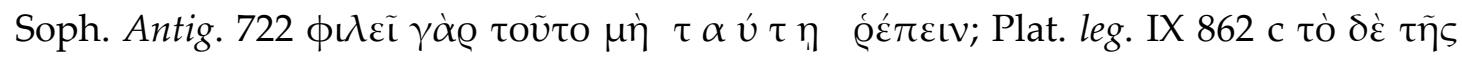

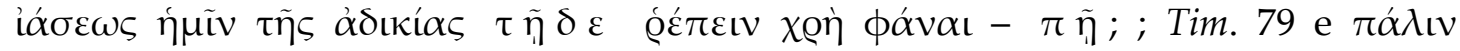

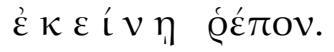

$169 \mathrm{f}$.

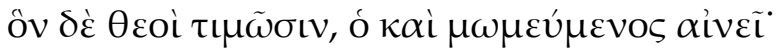

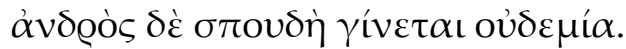

Radermacher (1938: $1 \mathrm{f}$.) hat in eindringlicher Argumentation gezeigt, daß es sich bei ó um den Artikel handelt und daß insbesondere die Variante ôv $\delta \dot{\varepsilon} \theta \varepsilon o \grave{~} \tau \iota \mu \tilde{\omega} \sigma$, ôv $\kappa \alpha \grave{i} \mu \omega \mu \varepsilon \dot{u} \mu \varepsilon v o \varsigma$ aìvẽ sprachlich nicht zu rechtfertigen ist. Überdies verbürgt ein Zitat aus Hesiod (der wohl auch Quelle des vorliegenden Spruchs ist) bei

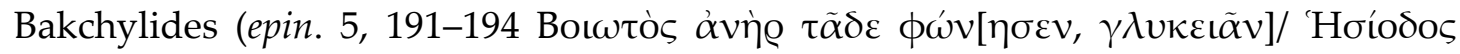

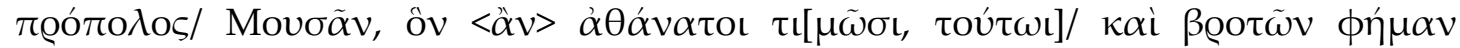
$\varepsilon \tilde{\pi}[\varepsilon \sigma \theta \alpha \mathrm{l}])$, daß die Rede sein muß von der Resonanz, die der von den Göttern Geschätzte in der Menschenwelt erhält. Alles Gesagte spricht gegen den in Wests

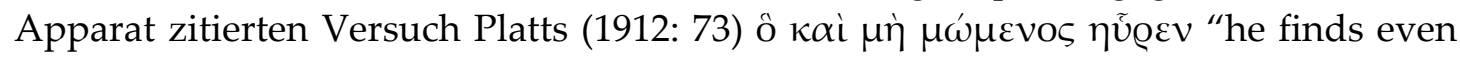
without seeking", der wieder ein Demonstrativum und überdies eine Aussage über ein aktives Handeln des Gottgeschätzten impliziert. Die überlieferte Antithese $\mu \omega \mu \varepsilon \hat{u} \mu \varepsilon v o \varsigma \alpha i v \varepsilon \tilde{\imath}$ bedarf schwerlich einer Änderung in zwei andere Begriffe.

Freilich bleibt in der von Radermacher verfestigten Deutung die Schwierig-

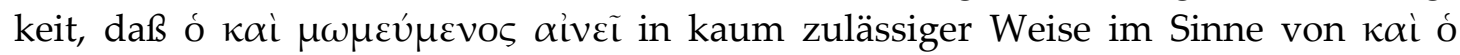

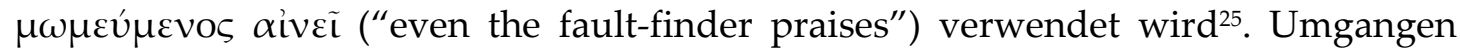
wird diese Schwierigkeit nur durch die Deutung von Hudson-Williams ("The man who is actually ( $\kappa \alpha$ í) blaming the favourite of the gods is at that very time praising him", vgl. Gerber (1970: 283) "a man even when he finds fault is praising ..."), die

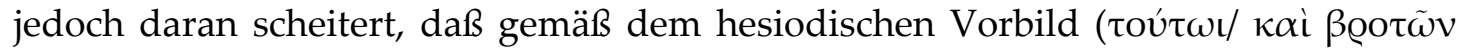
$\phi \eta \dot{\mu} \alpha v \varepsilon^{\prime} \pi[\varepsilon \sigma \theta \alpha \mathrm{\iota})$ von der faktischen Hochachtung des Gottgeliebten in der Menschenwelt, nicht von seinem verborgenen Wert, die Rede sein muß.

Demnach wird man für $\kappa \alpha i ́$ einen sich unmittelbar zu $\mu \omega \mu \varepsilon v ́ \mu \varepsilon v o \varsigma$ fügenden Begriff einsetzen: ó $\pi \tilde{\alpha} v \mu \omega \mu \varepsilon u ́ \mu \varepsilon v o \varsigma \alpha i v \varepsilon \tilde{\imath}$ "wen die Götter ehren, den lobt [auch] der, der [sonst] alles tadelt". Damit erhält die hesiodische Sentenz eine denkbar prägnante Schärfung.

$271-278$

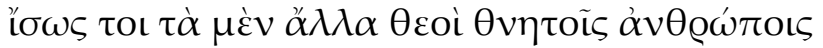

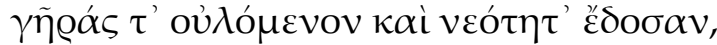

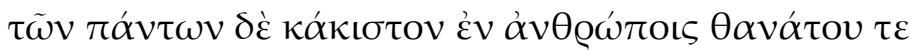

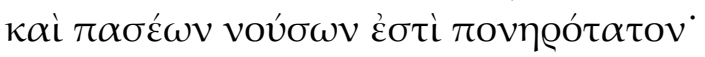

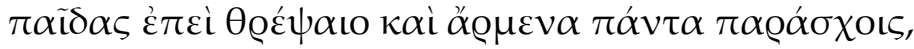

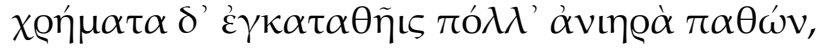

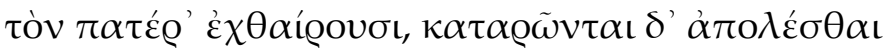

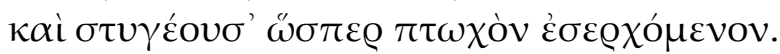

${ }^{25}$ Herausgestellt schon von Friedländer (1913: 590 Anm. 1), vgl. auch van Groningen z.St. 
Im zweiten Distichon läßt sich folgendermaßen eine Zuspitzung des pointierten

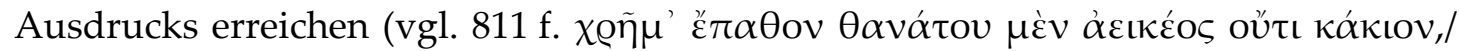

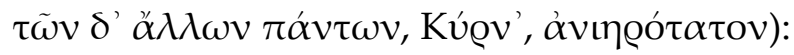

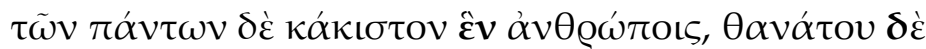

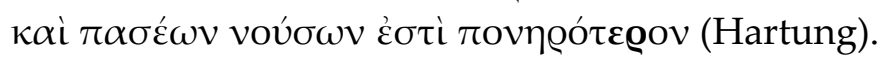

Unnötig kompliziert sind die in gleiche Richtung gehenden Versuche von

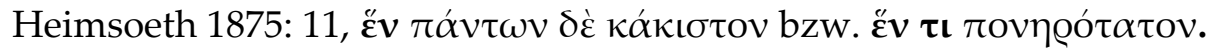

Der syntaktische Ablauf des dritten Distichons läßt sich einfacher glätten als

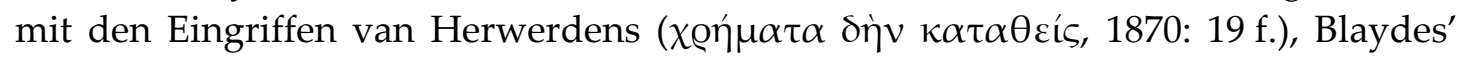

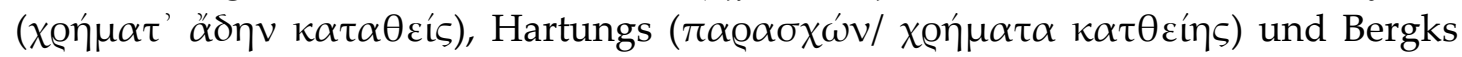

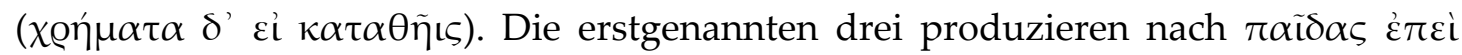
Ө९乏́ $\psi \alpha$ เo ein schwerfälliges zweites Glied mit einem finiten Verb und zwei untergeordneten Partizipien, wohingegen Bergks Konjektur einen unmotivierten Neuansatz

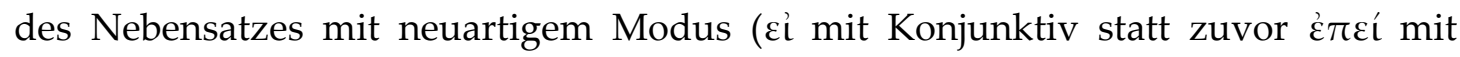

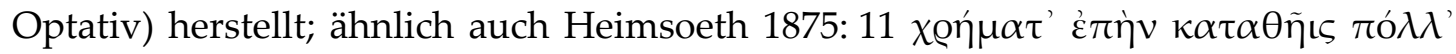

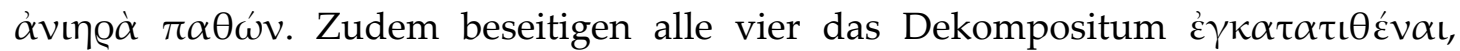
dessen ersten Bestandteil Friedländer überzeugend durch die Analogie zu $\omega \sigma \tau \varepsilon Q$

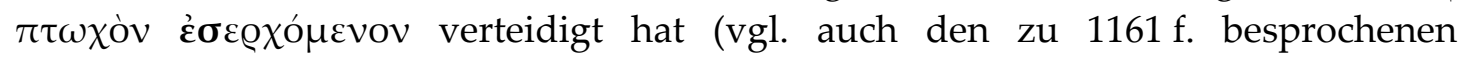

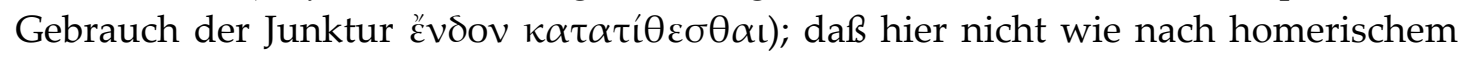
Sprachgebrauch das Medium des Verbs verwendet wird, ist nur natürlich, da der unglückliche Vater ja seine Reichtümer eben nicht zu seinem eigenen Nutzen, sondern für seine undankbaren Kinder hinterlegt. Nach den mit $\pi \alpha i \tilde{\delta} \alpha \varsigma$ bzw. $\alpha$ $\varrho \mu \varepsilon v \alpha$

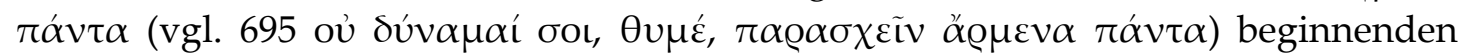
Satzsegmenten erwartet man nach dem Gesetz der wachsenden Glieder ein drittes längeres Segment, welches den alles entscheidenden Begriff $\chi \varrho \eta ́ \mu \alpha \tau \alpha$ nachdrücklich einführt; Sitzler (1878: 7) erkennt richtig, daß das dritte Glied bei- und nicht unter-

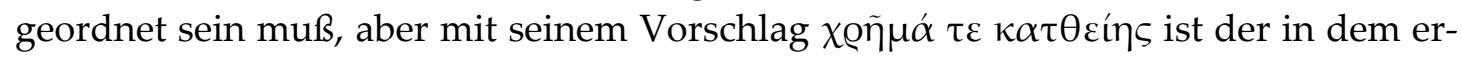
forderten Sinne "Geld" höchst ungebräuchliche Singular $\chi \varrho \tilde{\eta} \mu \alpha$ verbunden. Besser

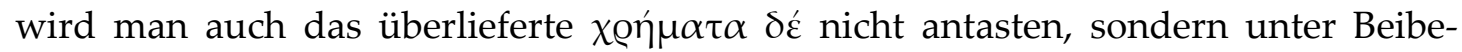
haltung der durch die papyrologische Überlieferung gesicherten Textbestandteile folgendes schreiben:

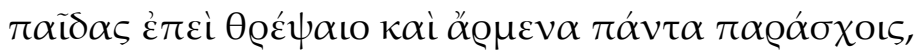

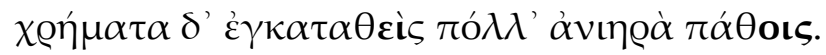

$319-322$

Kú

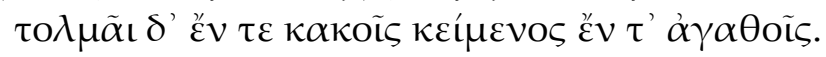

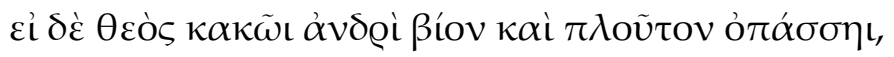

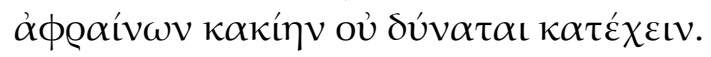

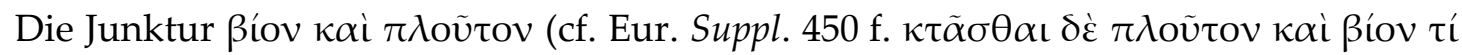

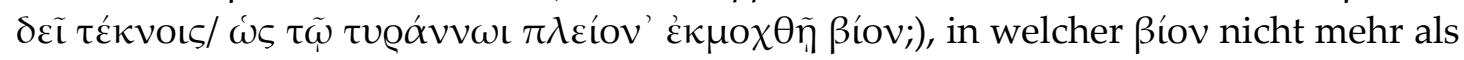
das zum Lebensunterhalt Erforderliche bezeichnet, erscheint zu schwach in einem Zusammenhang, wo es um die äußeren Machtmittel geht, welche eine Entfaltung

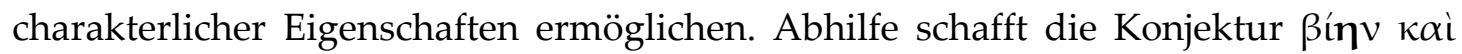




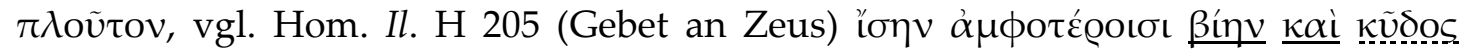
ót $\alpha \sigma \sigma o v$ (zitiert bei Hudson-Williams z.St., der jedoch keinerlei Textänderung

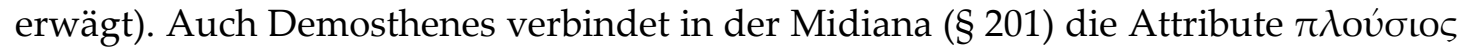
und ßía ıo in der Charakteristik seines reichen Kontrahenten.

$337-340$

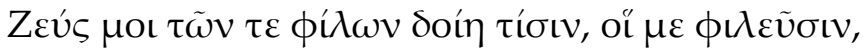

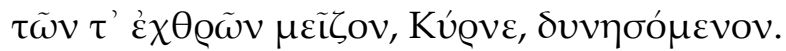

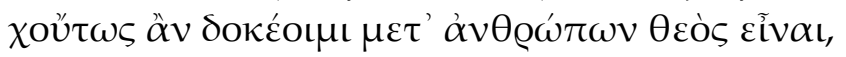

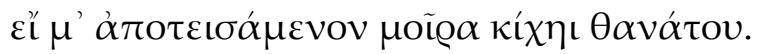

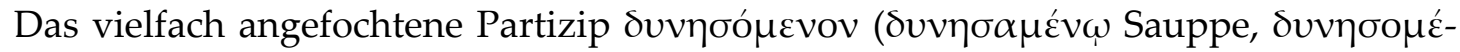

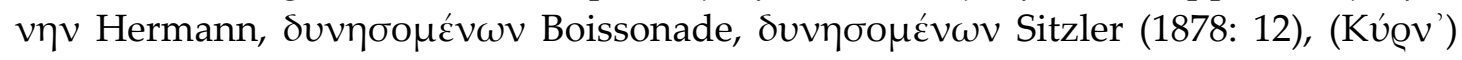

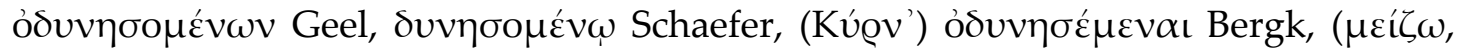

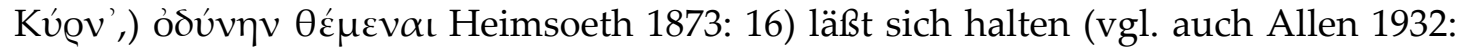
86; Carrière 1954: 53 f. nimmt unnötigerweise einen anakoluthisch auf $\mu$ oı bezüglichen Akkusativ an): “... und größere Macht als meine Feinde in der Zukunft”. Zu der (bei Thukydides zum Stilmerkmal gewordenen) substantivierten Verwendung des neutralen Partizips vgl. Kuehner/ Gerth I 267 f., besonders zum Partizip Futur

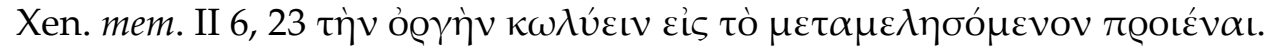

$351-354$

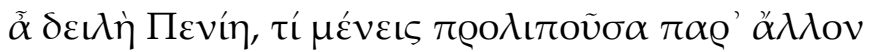

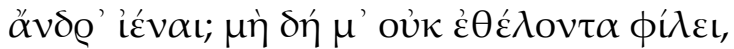

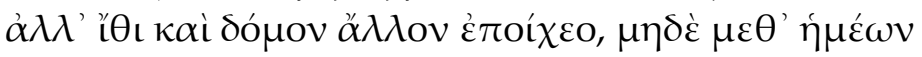

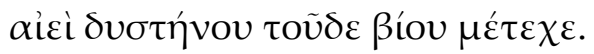

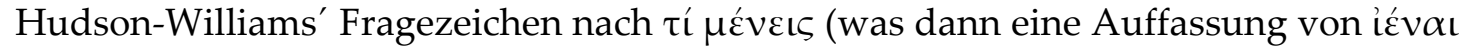
als imperativischen Infinitiv erforderlich macht) löst die Schwierigkeit nicht. $\pi \varrho \circ \lambda ı \pi \circ \tilde{v} \sigma \alpha$ erhält sein erfordertes Objekt (welches wegen der Antithese zu $\pi \alpha \varrho$ '

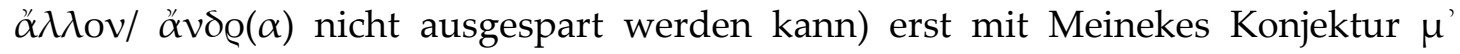

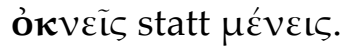

$401-406$

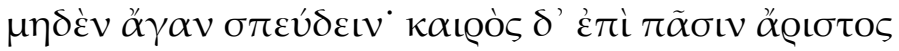

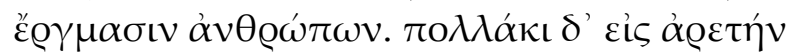

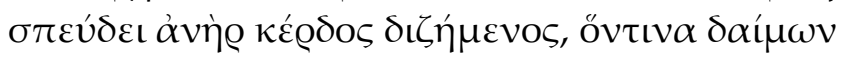

$$
\begin{aligned}
& \pi \varrho o ́ \phi \varrho \omega v \varepsilon i \varsigma \varsigma \mu \varepsilon \gamma \alpha \dot{\alpha} \lambda \eta v \dot{\alpha} \mu \pi \lambda \alpha \kappa i ́ \eta v \pi \alpha \varrho \alpha ́ \gamma \varepsilon l \text {, }
\end{aligned}
$$

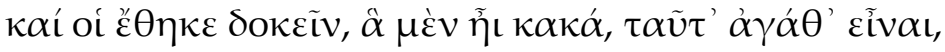

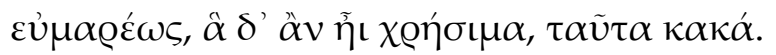

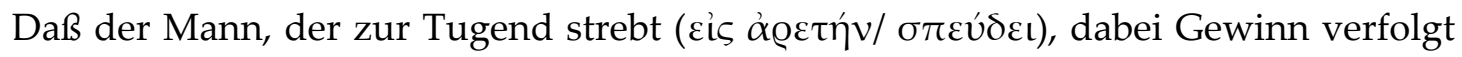

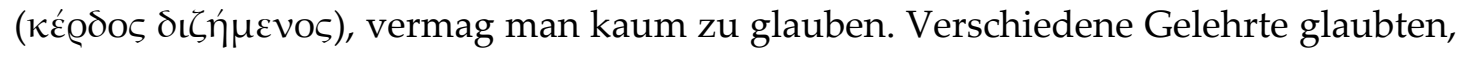
es gehe schon seit Beginn der mit $\pi$ o $\lambda \lambda \alpha \alpha_{\kappa} \iota \delta^{\prime}$ beginnenden Periode um einen 
habgierigen Bösen, der von der Gottheit zurecht ins Unglück gestürzt wird: Ahrens

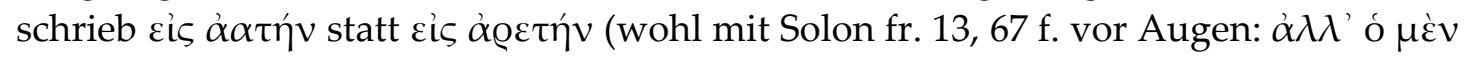

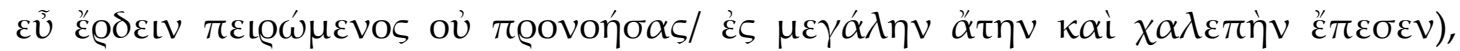

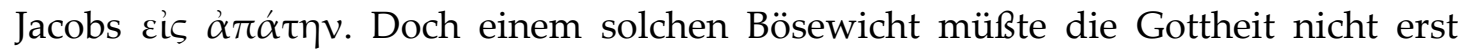

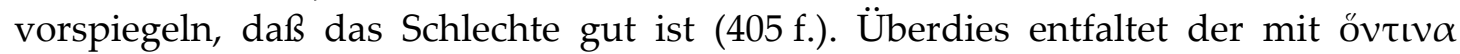

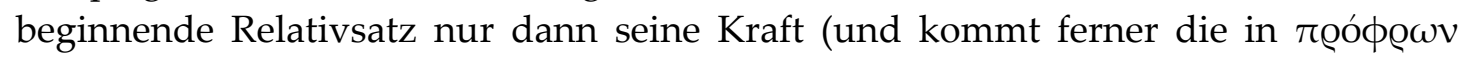
liegende Bosheit der Gottheit richtig heraus), wenn vor diesem Relativsatz nur von den guten Absichten des Menschen die Rede war. Dies läßt sich relativ leicht er-

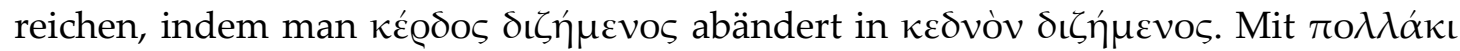

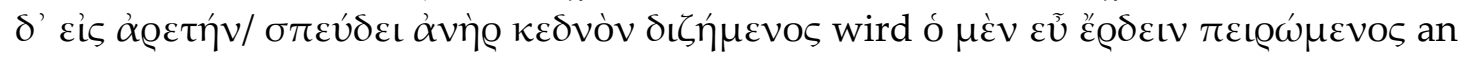
der oben zitierten Solon-Stelle umschrieben.

$421-424$

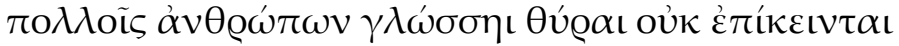

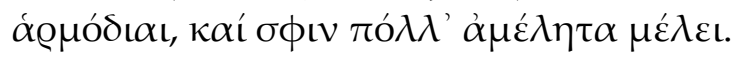

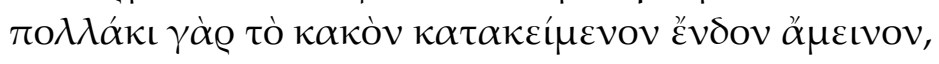

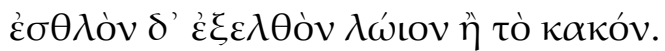

West beurteilt den Schlußteil von Vers 424 im Anschluß an Hudson-Williams als "finis fort. ab excerptore expletus". Bergk erwog dagegen im Apparat eine Umschreibung des Schlußpentameters: "Versus corruptus. Poeta videtur dixisse: bonum, quod divulgatum, plus nocuit, quam malum". Wenn der Sinn des Schlußverses im Gegenteil darauf hinausliefe, daß ein દ̇ $\sigma \theta \lambda$ óv ruhig ausgeplaudert werden darf (worauf viele Konjekturen abzielen), verstünde man nicht, was diese Aussage zum Gesamtthema des Gedichts (Bedeutung der Verschwiegenheit) beitrüge. Den von Bergk postulierten Sinn könnte man leicht in Form einer Litotes einführen (und dabei auch den

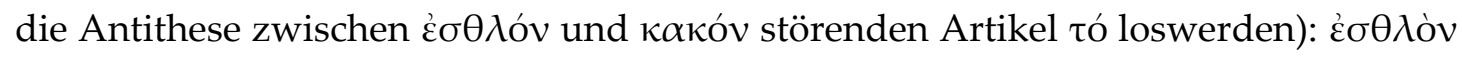

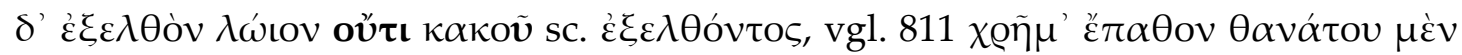

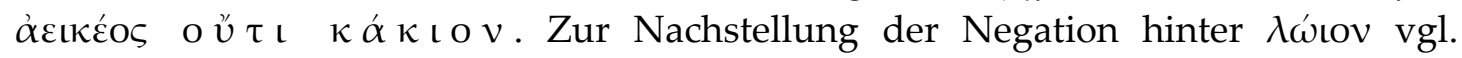
Kuehner/ Gerth II 179.

$571 \mathrm{f} .=1104 \mathrm{ab}$

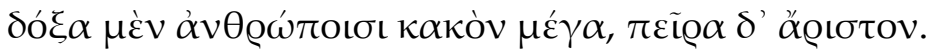

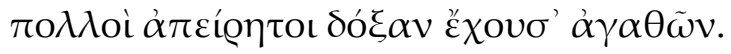

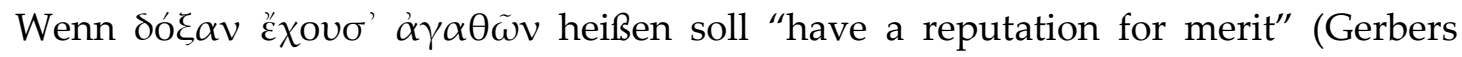
Übersetzung), so wird der entscheidende Gedanke offenkundig verfehlt, insofern hier $\delta o ́ \xi \alpha$ wie im Hexameter in der Bedeutung "bloße Meinung" als Gegenbegriff zu $\pi \varepsilon \tilde{\imath} \varrho \alpha$ verwendet sein muß. Unter dieser Voraussetzung müßte man annehmen, daß sich der Gedanke im Pentameter entweder auf philosophische Erkenntnis über $\dot{\alpha} \gamma \alpha \theta \dot{\alpha}$ ("viele haben ohne wirkliche Erfahrung eine bloße Meinung über die $\dot{\alpha} \gamma \alpha \theta \alpha^{\prime \prime}$ ) oder auf persönliche Zukunftserwartungen verengt ("many imagine joys they've never known", Gerber 1999: 255 Anm. 1 nach West 1974: 156). Eine solche Verengung des Gedankens schadet jedoch nur der Praegnanz des Epigramms; entsprechendes gälte auch für das in $1104 \mathrm{~b}$ als A-Variante überlieferte $\dot{\alpha} \gamma \alpha \theta$ oí (dort aufgenommen bei van Groningen, auch von Bergk zu 572 als "non male" bewertet), 
welches das Subjekt $\pi$ o $\lambda \lambda$ oó in völlig müßiger Weise erweitern würde. Eine konjekturale Verbesserung ${ }^{26}$ ergibt sich, wenn man den Pentameter abändert zu $\pi \mathrm{o} \lambda$ 入oì

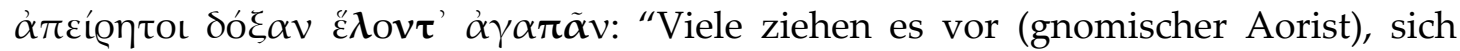
ohne Erfahrung mit bloßer Meinung zufriedenzugeben". Diese (im Attischen verbreitete) Bedeutung von $\dot{\alpha} \gamma \alpha \pi \tilde{\alpha} v$ ist bereits homerisch, vgl. Od. $\phi 289 \mathrm{f}$.

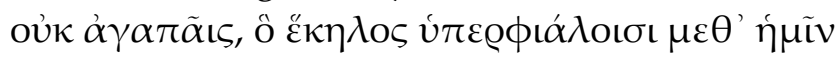

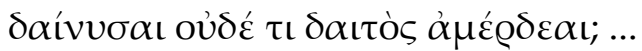

$591-594$

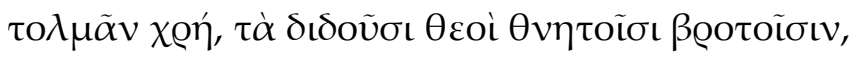

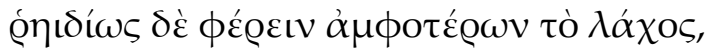

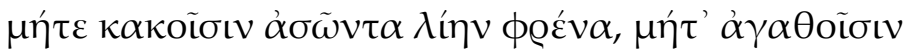

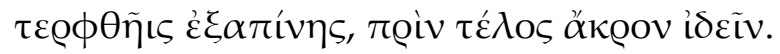

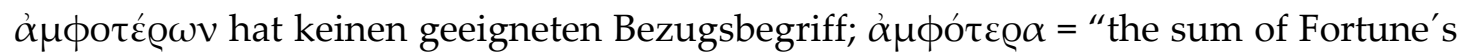
chances on either side" (Hudson-Williams) ist dem Text nicht gerade leicht abzugewinnen (an der zitierten Parallelstelle 934 sind die mit $\dot{\alpha} \mu \phi o ́ \tau \varepsilon \varrho \alpha$ bezeichneten

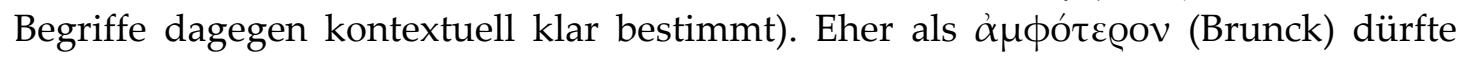

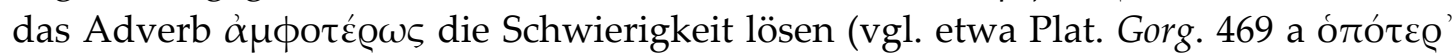

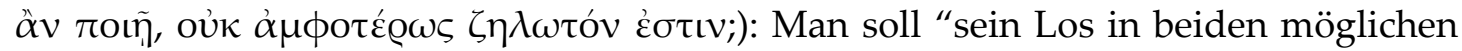
Fällen (im Glück wie im Unglück) mit Leichtigkeit tragen".

$607-610$

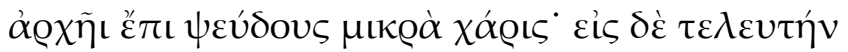

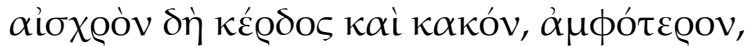

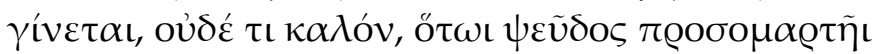

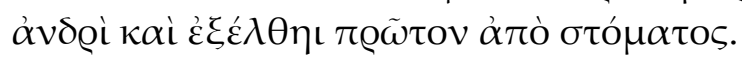

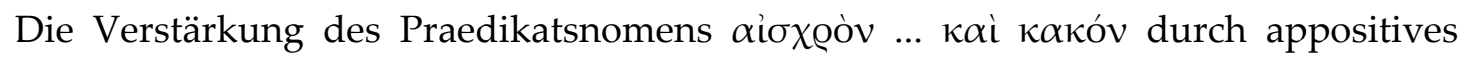

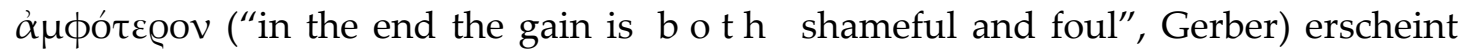
verdächtig. Passender wäre eine Bemerkung über die letztlich unvermeidliche Ent-

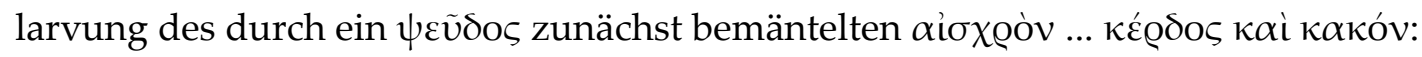

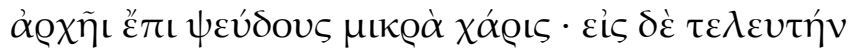

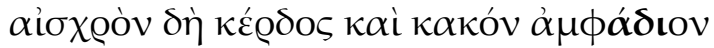

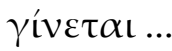

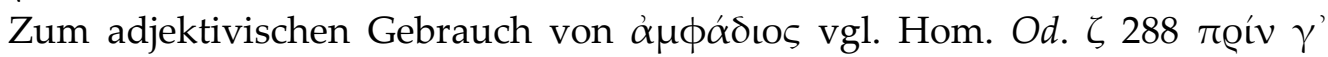

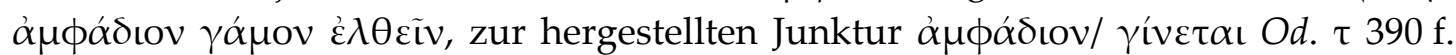

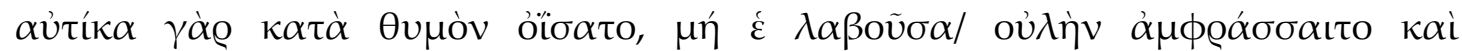

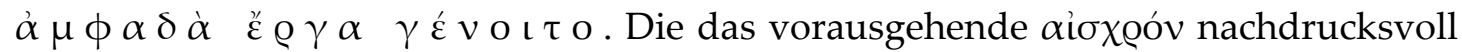
betonende Partikel $\delta \eta \dot{~ s t e l l t ~ d i e ~ K o r r e k t u r ~ d e s ~ t r u ̈ g e r i s c h e n ~ A n f a n g s e i n d r u c k s ~(~} \dot{\alpha} \varrho \tilde{\eta} \iota$

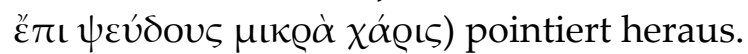

Im folgenden wird mit der idiomatischen Anakoluthie $\kappa \alpha \lambda$ óv, ö $\tau \omega$ (vgl.

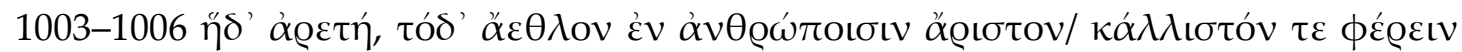

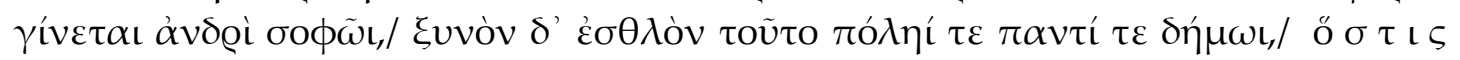

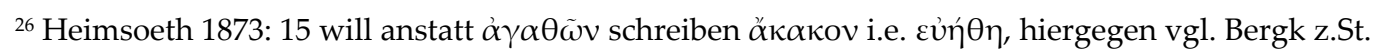




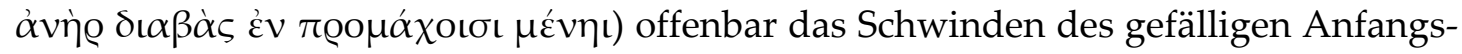
eindrucks des $\psi \varepsilon \tilde{v} \delta o \varsigma$ und seine dauerhaften Folgen beschrieben: Wem einmal ein solches $\psi \varepsilon \tilde{v} \delta$ o $\varsigma$ von den Lippen gegangen ist, der wird es nicht mehr los. Diesen Gedanken ("wer einmal lügt, dem glaubt man nie mehr") und den temporalen Aspekt, daß der gefällige Anfangseindruck nicht mehr vorhanden ist, kann man mit folgender gebesserter Textfassung einigermaßen praegnant herausbringen:

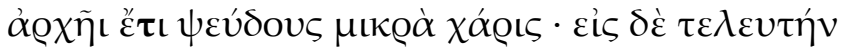

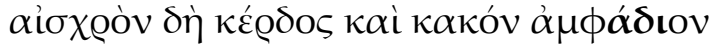

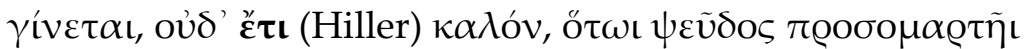

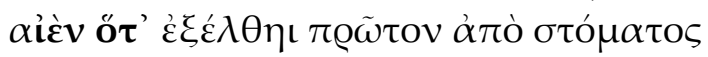

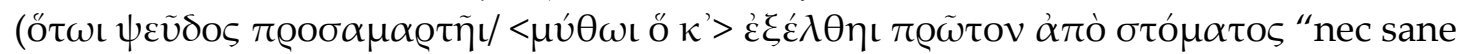
pulchrum est, si verbo quod primum ori exierit adest mendacium" van Herwerden 1884: 299). “Dem Anfang einer Lüge wohnt noch eine gewisse Gefälligkeit inne. Am Schluß aber wird das häßliche und böse Gewinnstreben offenbar, und es ist nicht mehr schön, wenn einem die Lüge immerzu folgt, seit sie einem erstmalig aus dem Mund getreten ist".

$641-644$

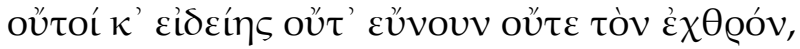

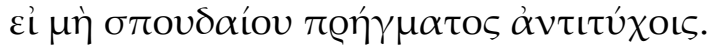

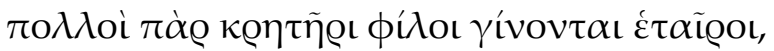

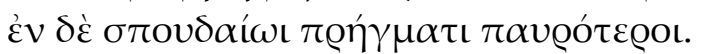

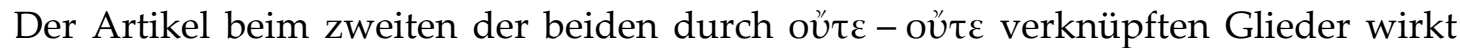
sonderbar. Emperius änderte $\tau o ̀ v$ in $\tau$ oı (Carrière hat stattdessen $\mu \varepsilon ́ v$ ohne Angabe

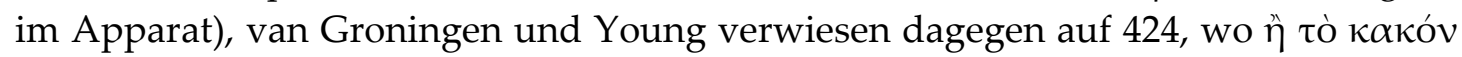
nach dem oben Gesagten ebenfalls zweifelhaft überliefert ist. Hinzukommt, daß

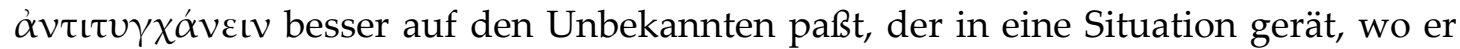
seine wahren Eigenschaften offenbart, als auf den Angesprochenen, der eine solche Situation nicht zufällig antrifft, sondern geradezu herbeisehnen müßte. Vor allem schließt sich an den Gedanken, daß der Unbekannte in eine ihn offenbarende

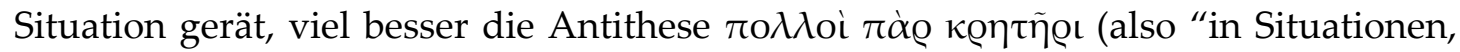
die nicht entlarven") an. Beide Schwierigkeiten lösen sich, wenn man schreibt:

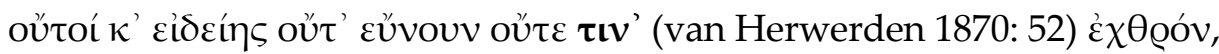

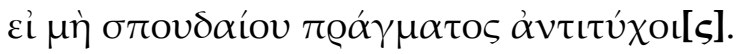

So erhält auch $\dot{\alpha} v \tau t-$ eine gewisse Pointe: "Man kann erst über ihn urteilen, wenn er

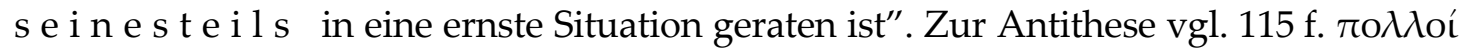

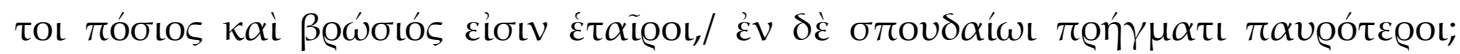

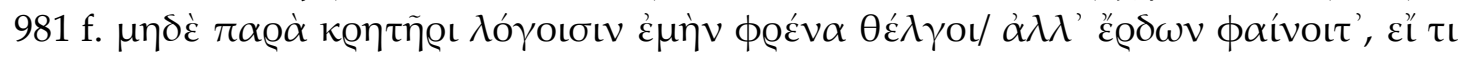

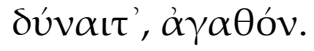

$659-662$

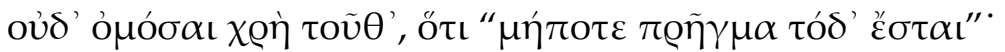

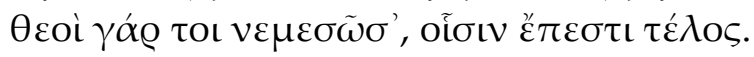

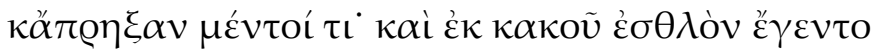

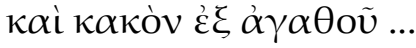




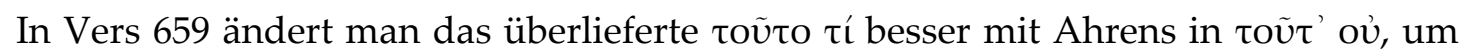
mit dem idiomatischen oủ $\mu$ ń die hybrishafte Überheblichkeit des Sprechers zu pronouncieren (vgl. Kroll 1955: 79).

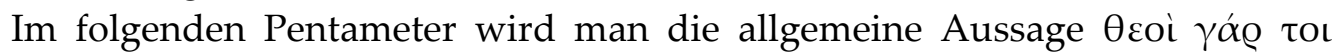

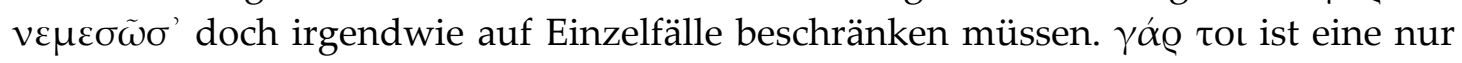
durch Camerarius bezeugte Variante für sonst überliefertes $\gamma \alpha \dot{\varrho} \varrho \varepsilon$. Die nötige Einschränkung ergibt sich (zugleich mit einem passenden drohenden Unterton) mit $\theta \varepsilon o i$

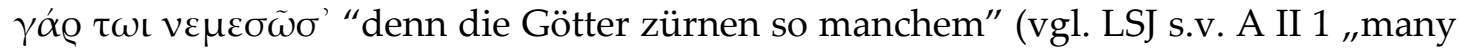
a one“); eine indefinite Variation von Hes. op. $741 \tau \tilde{\omega} \delta \dot{\varepsilon} \theta \varepsilon o \grave{~} v \varepsilon \mu \varepsilon \sigma \tilde{\omega} \sigma \iota \kappa \alpha \grave{\iota} \alpha \not \lambda \gamma \varepsilon \alpha$ $\delta \tilde{\omega} \kappa \alpha v$ ò $\pi \dot{\sigma} \sigma \sigma \omega$.

Schließlich erscheint auch Wests Konjektur in Vers 661 für überliefertes $\kappa \alpha \grave{~}$ $\pi \varrho \tilde{\eta} \xi \alpha \mathrm{l}$, die einen neuen Hauptsatz einführt ${ }^{27}$, fraglich (1974: 157: “The gods not only dislike assumptions about the future, which is in their hands, they confute them effectively"; Gerber übersetzt: "They act, what's more"). Eine epexegetische

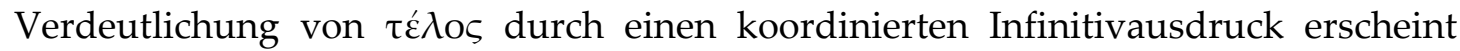

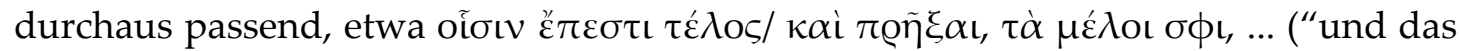
umzusetzen, was ihnen am Herzen liegen mag"), vgl. 1048 ă $\sigma \sigma \alpha \delta$ '

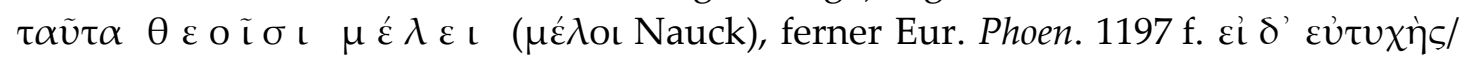

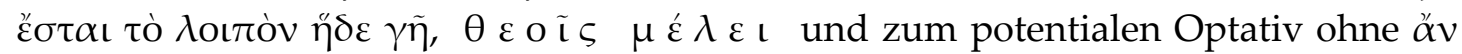
in Relativsätzen vg. Kuehner/Gerth I 252 f. (West 1974: 150) und II 429. Als

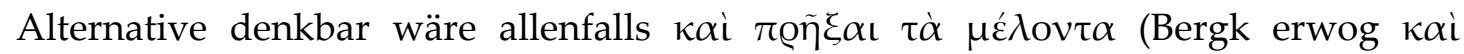
$\pi \varrho \tilde{\eta} \xi \alpha \iota \mu \varepsilon \lambda \varepsilon \dot{\varepsilon} \omega \omega$, aber an den Imperativ $\mu \varepsilon \lambda \varepsilon \dot{\varepsilon} \tau \omega$ fügt sich die schildernde Fort-

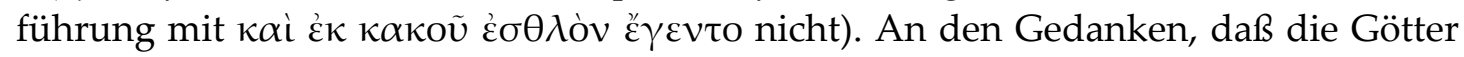
die Macht haben, alles ihnen Beliebende in die Tat umzusetzen, schließen sich mit

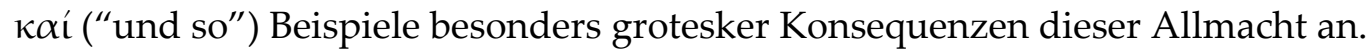

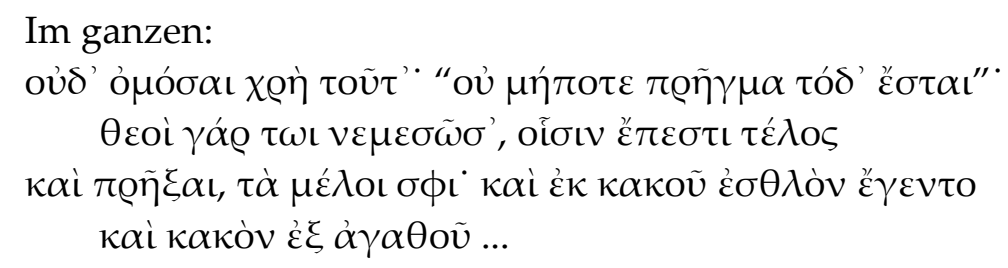

683-686

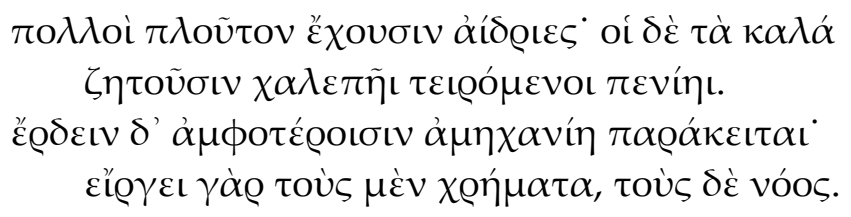

Die etwas befremdliche Verwendung von elo $\gamma \varepsilon \iota$ in dem Sinne von "hindert durch sein Nichtvorhandensein" (vgl. Hudson-Williams z.St.: "res pro rei defectu") beruht möglicherweise aus einem Influenzfehler aus dem darüberstehenden ع̌ dann ein ursprüngliches $\lambda \varepsilon i ́ r \varepsilon \iota$ ("geht aus", "ermangelt") zum Opfer gefallen sein könnte. $\lambda \varepsilon i ́ \pi \varepsilon \iota$ paßt gut zum Gedanken der $\alpha \dot{\mu} \eta \chi \alpha v i ́ \eta$.

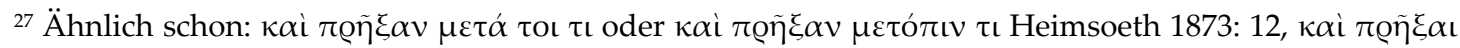

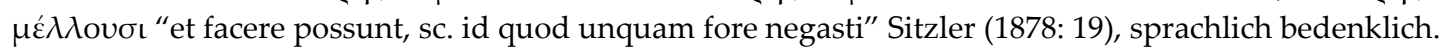


$715 \mathrm{f}$.

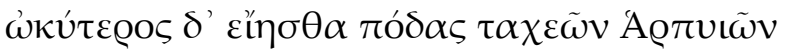

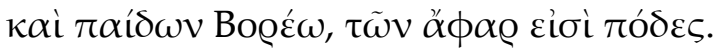

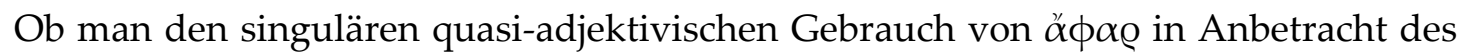

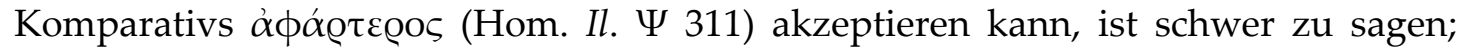

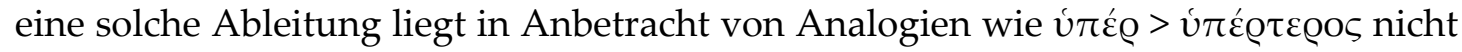
gerade fern und besagt wenig über die Verwendung des zugrundeliegenden

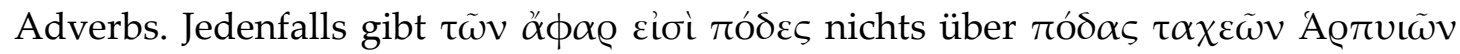
Hinausgehendes, was den hypothetisch gesetzten Gedanken enormer Schnelligkeit

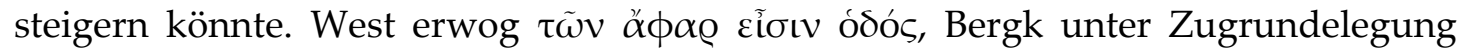

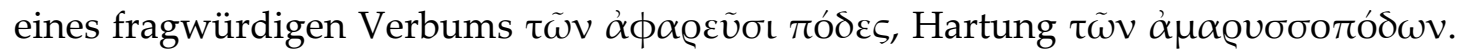
Am besten käme die Besonderheit der Boreaden wohl zum Ausdruck durch ein $\tau \tilde{\omega} v$

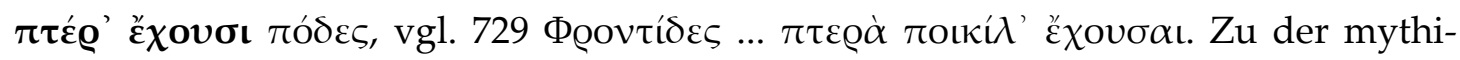
schen Version, daß die Boreaden Flügel an ihren Füßen haben (meist werden diese an ihrem Rücken lokalisiert, vgl. Roscher I 798/ 800), vgl. Apoll. Rhod. Arg. I 219-221

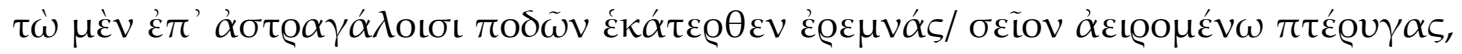

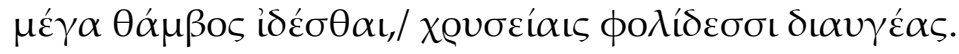

\section{$769-772$}

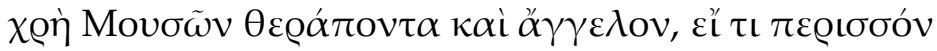

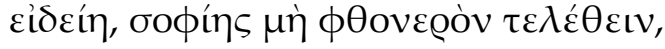

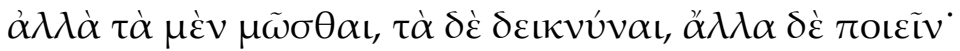

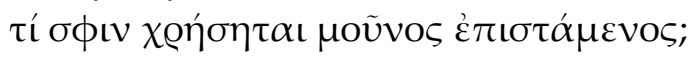

Eine Zusammenstellung der bisherigen, wenig erfolgreichen Versuche, die drei

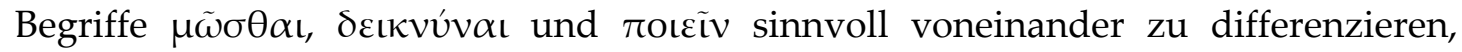
bietet Leonard E. Woodbury (1991: 483-490). Sein eigener Versuch (a.a.O. 488: "first inspiration and discovery, then public demonstration and finally, what remains, the production of verses") bringt einen Aspekt zeitlicher Abfolge hinein, der dem Griechischen $\tau \dot{\alpha} \mu \grave{\varepsilon} v \ldots, \tau \dot{\alpha} \delta \dot{\varepsilon} \ldots, \not \ddot{\alpha} \lambda \lambda \alpha \delta \dot{\varepsilon} \ldots$ völlig fremd ist.

West (1974: 158) faßt $\mu \tilde{\omega} \sigma \theta \alpha \mathrm{\iota}$ in der Bedeutung "'meditate', with a suggestion

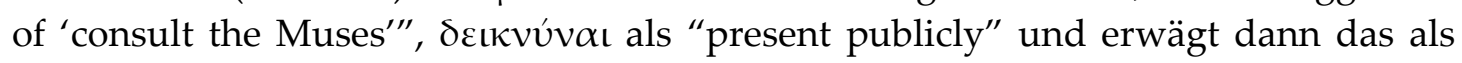

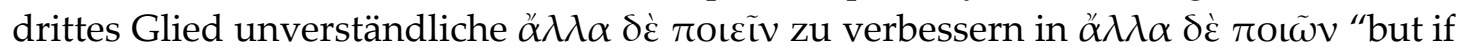
he does otherwise", was jedoch eine ziemlich müßige Vorwegnahme von noṽvos

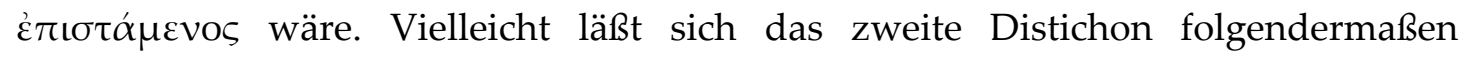
emendieren:

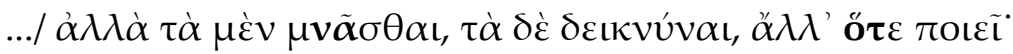

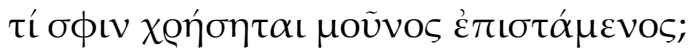

“.. sondern er muß (von seinem Wissen) sich an manches (beiläufig) erinnern, manches aber (exkursartig) vorführen, während er (eigentlich) über anderes dichtet". Der

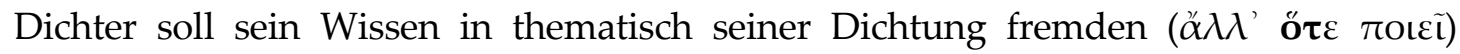

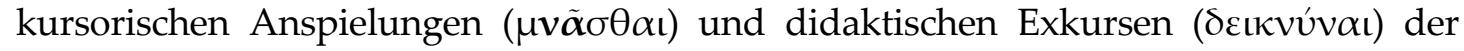
Öffentlichkeit präsentieren. 
$957 \mathrm{f}$.

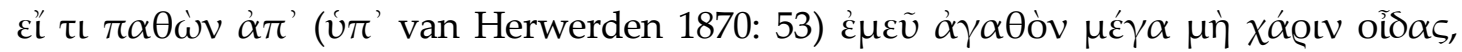

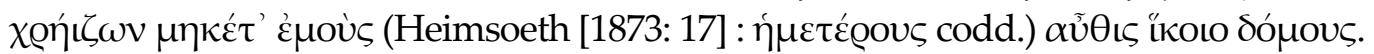

Es geht nicht um das Erfahren besonders großer Wohltaten, sondern einfach "ums Prinzip". Insofern wird man $\mu \varepsilon ́ \gamma \alpha$ in adverbiales $\mu \varepsilon \tau \alpha \dot{\alpha}$ (vgl. zu 923-930) abändern: "wenn du, nachdem du von mir etwas Gutes erfahren hast, h i n t e r h e r keinen Dank weißt".

973-978

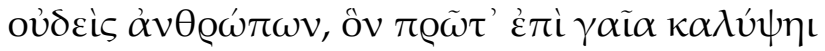

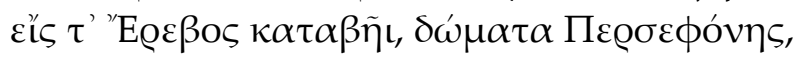

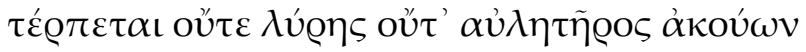

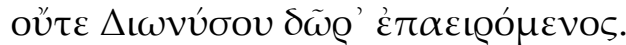

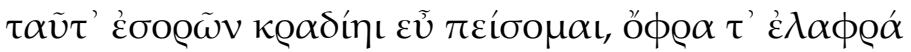

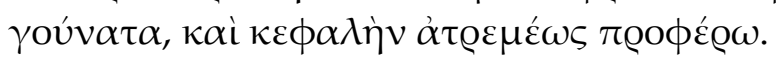

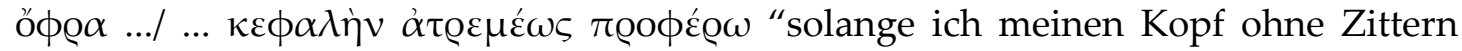

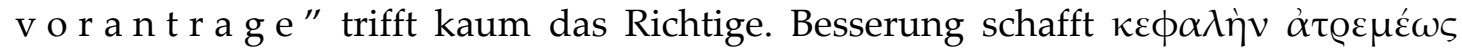
óф@’ ع́ $\chi \omega$ (Bergk versucht die Überlieferung mit der fragwürdigen Analogie zu

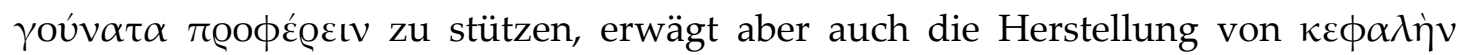

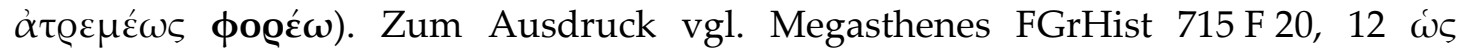

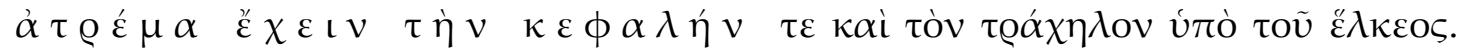
Zur Kurzmessung der ersten Silbe von öф@ $\alpha$ vgl. 1143; zu der Nachstellung von őф@ $\alpha$ (verbunden mit unmittelbarer Juxtaposition neben dem Verb) Hom. Od. $\lambda 96$

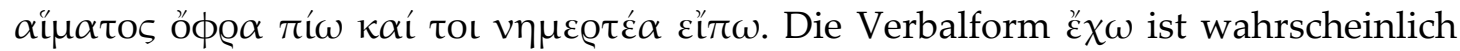
Konjunktiv, zum bloßen Konjunktiv ohne Modalpartikel in solchen Temporalsätzen vgl. Kuehner/ Gerth II 449 f. Anm. 4; zur Ellipse des konjunktivischen Auxiliarverbs Hom. Il. $\Lambda 477$ (s.u.). Der zweigliedrige "solange"-Satz wie bei Hom. Il. I 609 f. = K

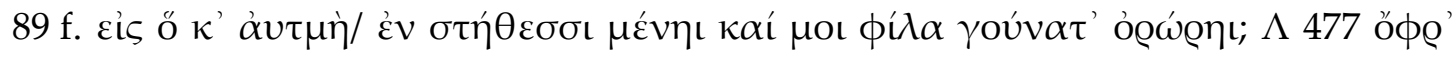

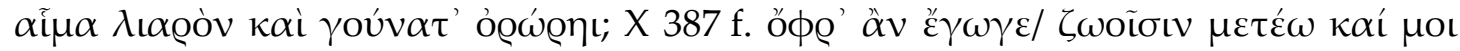

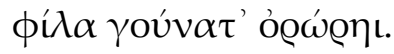

983-988

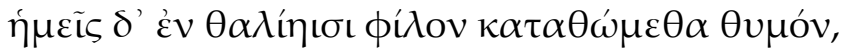

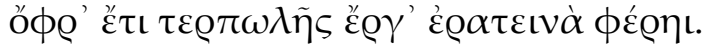

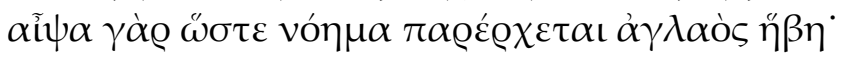

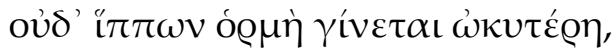

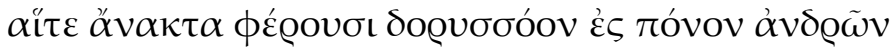

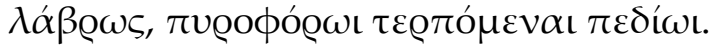

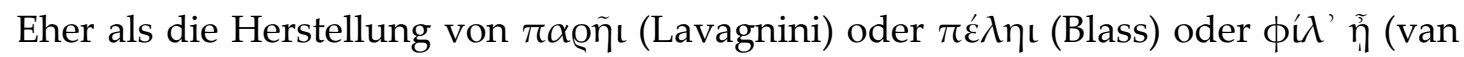

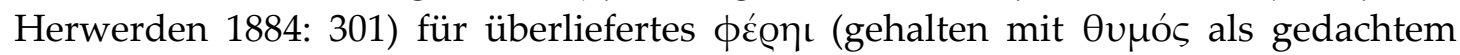


Subjekt bei Hudson-Williams z.St. und Garzya 1957: 216) empfiehlt sich öфQ' étı ...

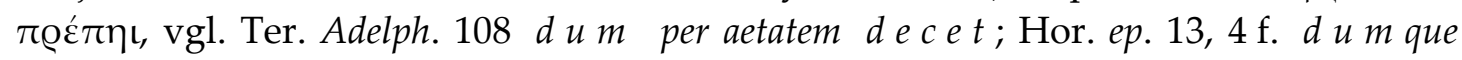
virent genual Et d e ce $t$, obducta solvatur fronte senectus.

Im Schlußvers kommt das Vorwärtsdrängen der Pferde erst richtig zum

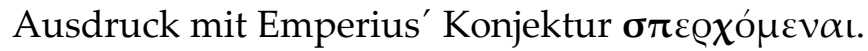

997-1002

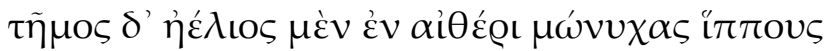

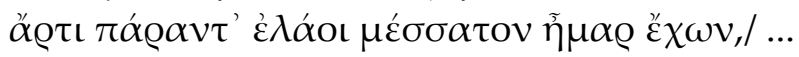

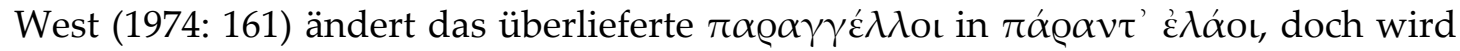
man den in der Tat erforderlichen Begriff $\dot{\varepsilon} \lambda \alpha u ́ v \varepsilon เ v$ eher an anderer Stelle einfügen:

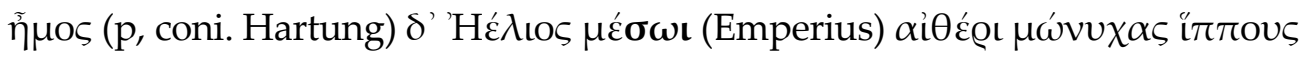

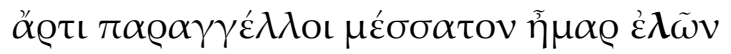

"sobald die Tatsache, daß die Sonne ihre Pferde mitten über den Himmel treibt, gerade den Mittag ankündigt". Zum Gebrauch von $\dot{\varepsilon} \lambda \tilde{\omega} v$ als Partizip Praesens in bezug auf den Sonnengott vgl. Timon von Phlius, SH 841, 6 ôs $\pi \varepsilon @ i ̀ ~ \pi \tilde{\alpha} \sigma \alpha v$ $\dot{\varepsilon} \lambda \tilde{\omega} v \quad \gamma \alpha \tilde{\mathrm{i} \alpha} v \dot{\alpha} v \alpha \sigma \tau \varrho \varepsilon ́ \phi \varepsilon \tau \alpha$. Die Herstellung von $\dot{\varepsilon} \boldsymbol{\lambda} \tilde{\omega} v$ wird möglicherweise bereits antizipiert in der lateinischen Übersetzung von Franz Kraneveld (vgl. Ijsewijn

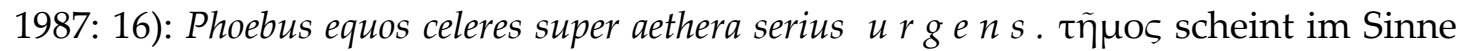
von $\tilde{\eta} \mu$ os gebraucht zu sein, wie Allen (1940: 214) annimmt.

1049-1054

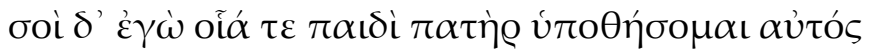

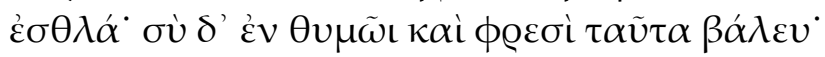

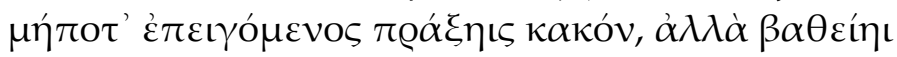

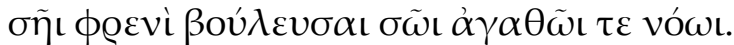

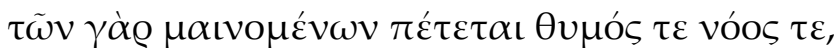

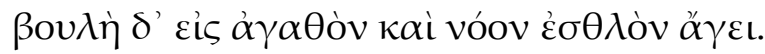

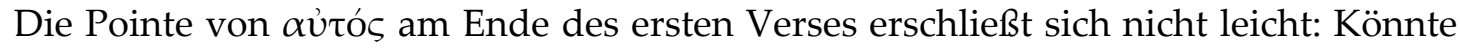
der Vater die Beratung seines Sohnes etwa an einen dritten delegieren? Ein pointiertes Polyptoton ganz im Sinne der aristokratischen Tendenz der Theognideen

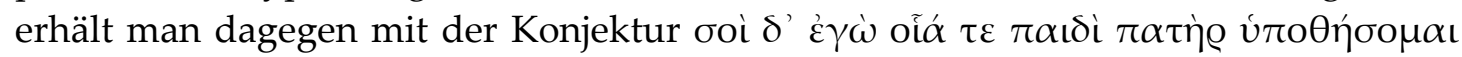

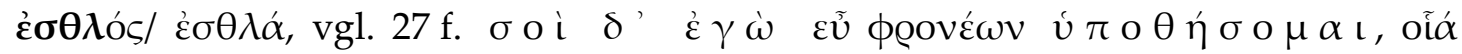

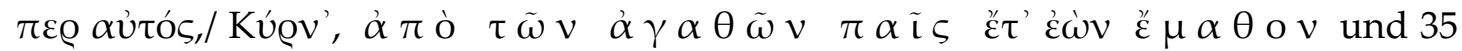

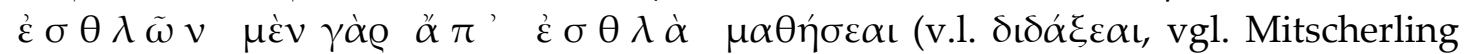
1982: 468 f.). Dieses Polyptoton ist bereits homerisch, Il. $\Xi 381 \mathrm{f}$. oiðó $\mu \varepsilon v o r ~ \delta ’ \dot{\varepsilon} \pi \mathrm{i}$

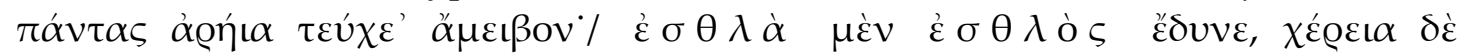

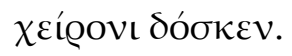

Was das Schlußdistichon anbelangt, so findet man in den Apparaten immer

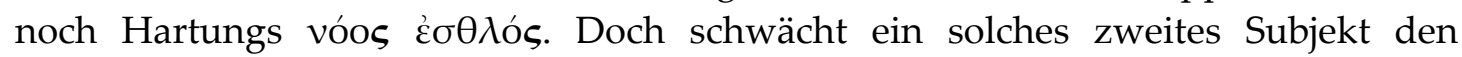

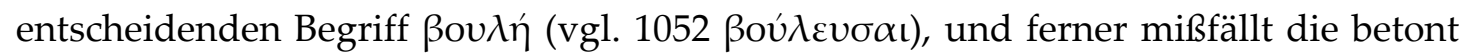
qualitative Verwendung von vóos nach der wertneutralen Verwendung desselben Wortes in Vers 1053. Beide Bedenken betreffen auch Bergks Versuch, nur vóov in 


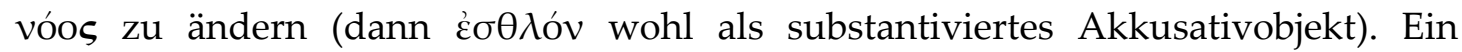

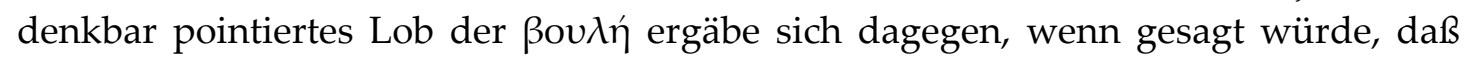

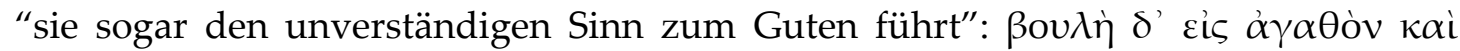

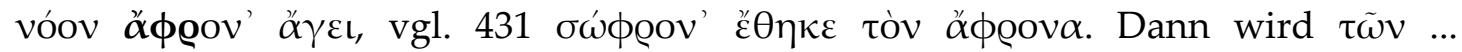

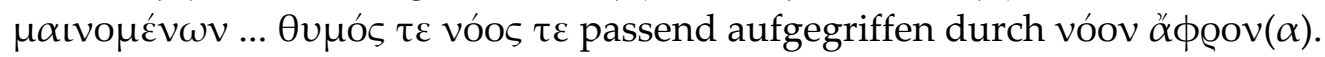

1075-1078

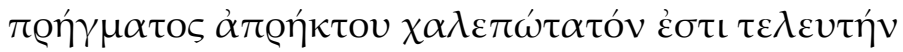

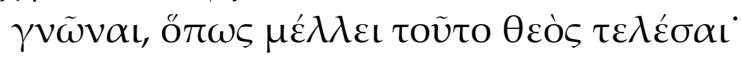

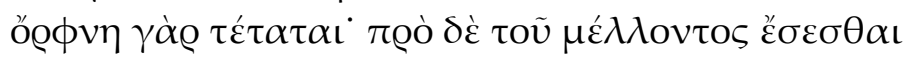

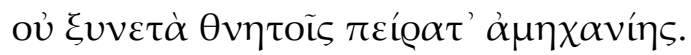

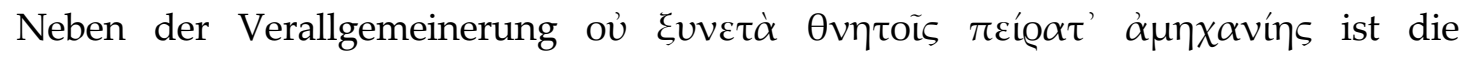

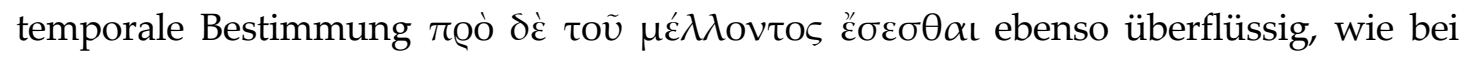

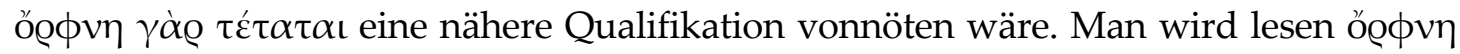

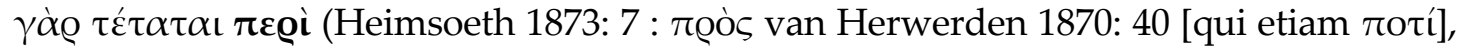

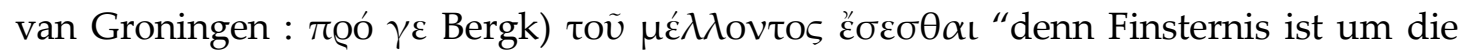

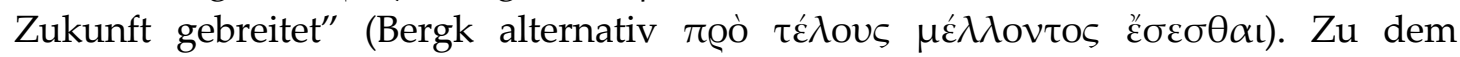
epischen Gebrauch von $\pi \varepsilon$ Qí cum gen. in dem Sinne "um" vgl. LSJ s.v. I 1, besonders

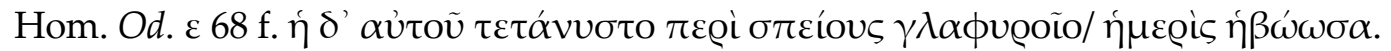

Ferner fragt man sich, wie es über die "Grenzen der Unmöglichkeit" ( $\pi \varepsilon \dot{L} \varrho \alpha \tau$ '

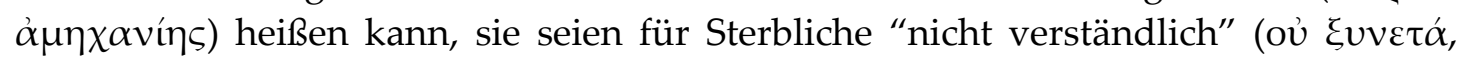
"mortals cannot comprehend the limits of their helplessness", Gerber). Anderswo werden diese "Grenzen" als etwas den Einzelnen Einschränkendes erwähnt (139 f.

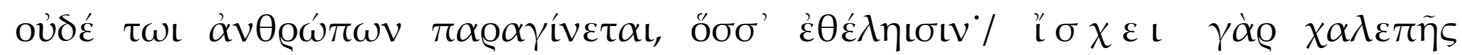

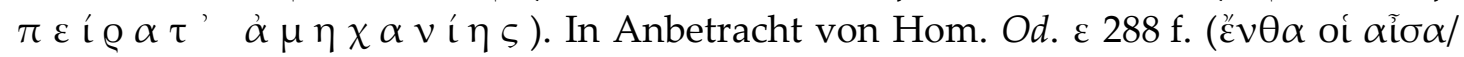

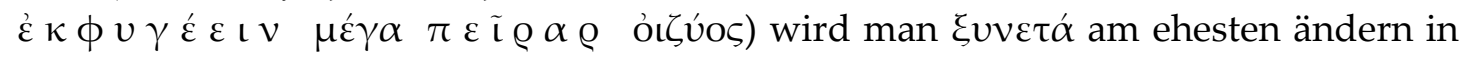

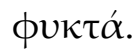

$1115 \mathrm{f}$.

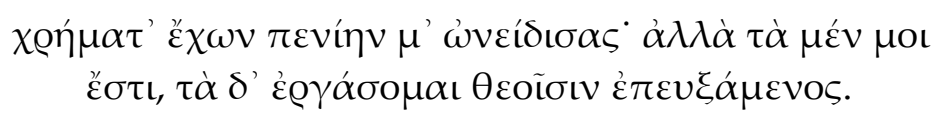

Mit "but I have some things and other things I'll earn" (Gerber) wird der Vorwurf

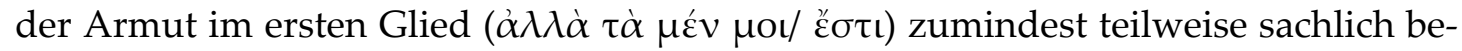
stritten. Eine rein argumentative Erwiderung ergäbe sich, wenn man schreibt $\dot{\alpha} \lambda \lambda \dot{\alpha}$

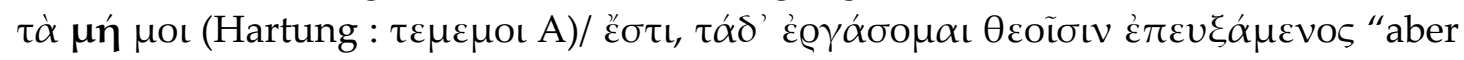
wenn ich etwas nicht besitze, werde ich es mir nach einem Gebet an die Götter erarbeiten". Insofern $\tau \dot{\alpha} \mu \dot{\varepsilon} v \mu \mathrm{or}$ nicht einhellig überliefert ist, geht Hudson-Williams' Bemerkung, Hartungs Konjektur sei "not needed", ins Leere.

$1129-1132$

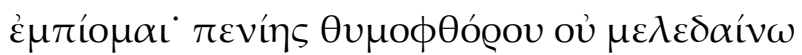

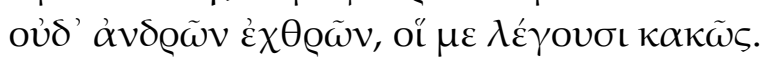




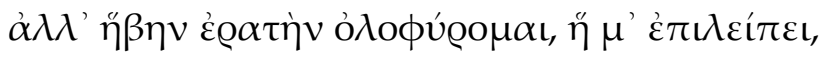

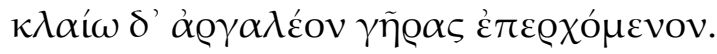

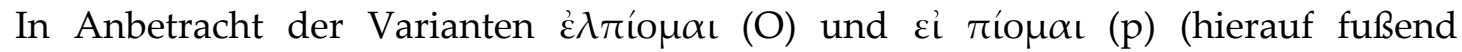

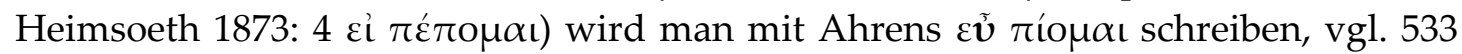

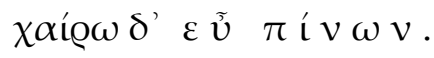

$1139 \mathrm{f}$.

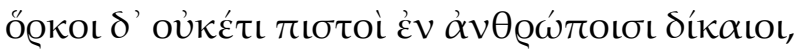

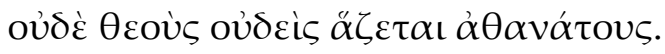

Die Überlieferung stellt den Leser vor die schwierige Frage, welches der beiden Ad-

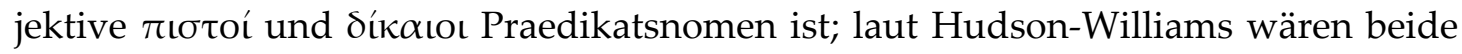
Attribute, nach van Groningen wäre $\tau$ ı $\tau \tau o$ vó von der danebenstehenden Negation

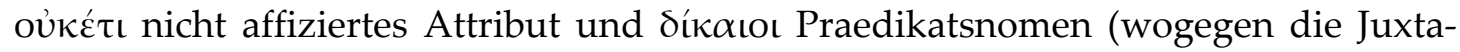

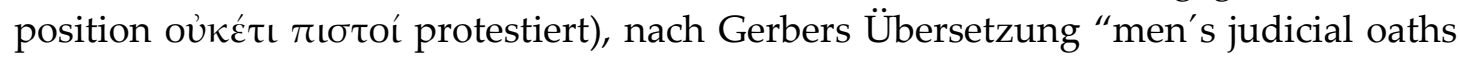
are no longer to be trusted" verhielte es sich umgekehrt (in welchem Falle das starke

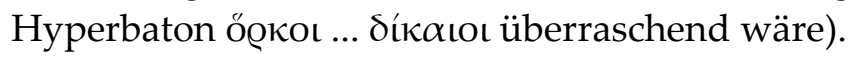

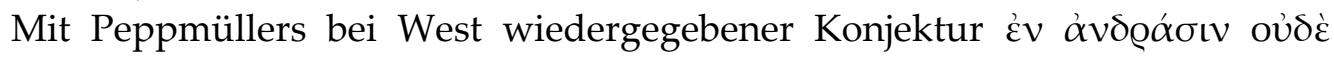

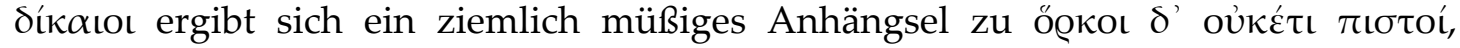

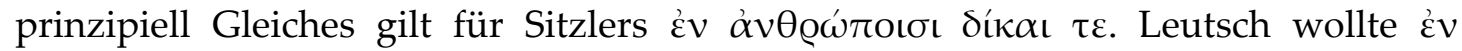

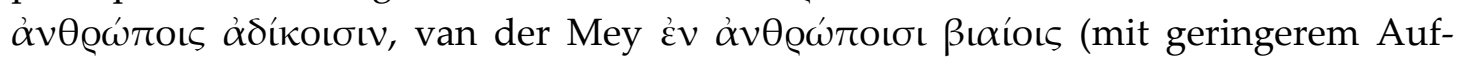
wand könnte man unter der Annahme, daß die Negation oủkétı noch auf den Prae-

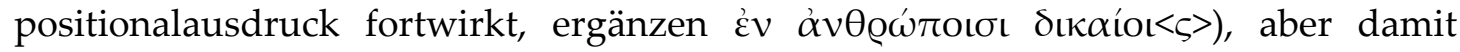
würde der Gedanke der Entartung der Menschen selbst aus dem nächsten Distichon vorweggenommen. Bergk bemerkt richtig: "locus alia medicina eget".

Einen kompakten Ausdruck, der das Erlöschen der Kraft der Eide zugleich als

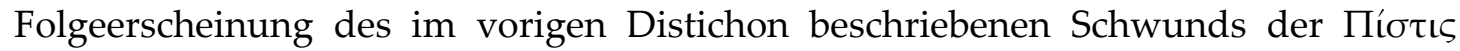

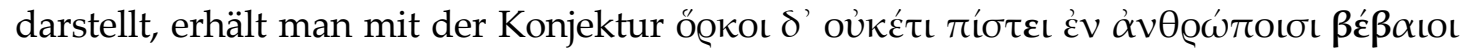

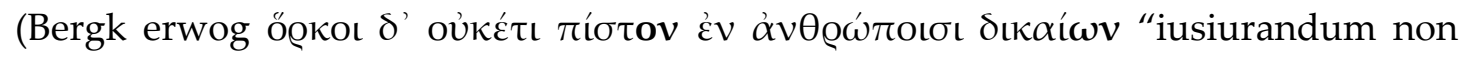

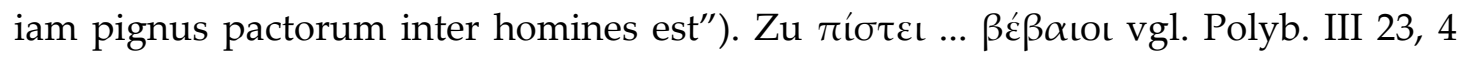

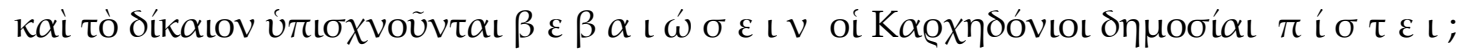

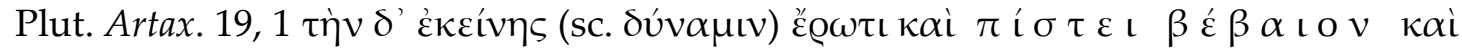
iơv@óv.

$1141 \mathrm{f}$.

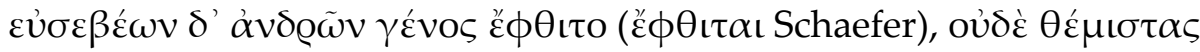

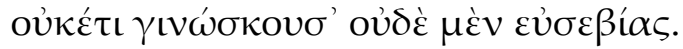

Um die Wiederholung zu vermeiden, schrieb van Herwerden (1884: 302 und 1870:

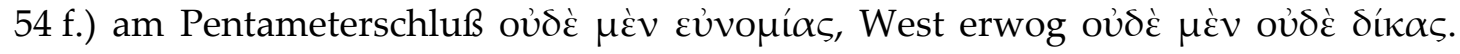
Kräftiger als eine zweite nicht erkannte Instanz wirkt aber wohl die faktische

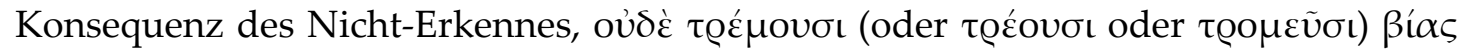
"und sie schaudern nicht vor Gewalttaten". Zur (in den Theognideen verhältnis- 
mäßig häufigen) Correptio attica vgl. West 1974: 114; in diesem Gedicht 1143 ( $\dot{\alpha} \lambda \lambda^{\prime}$

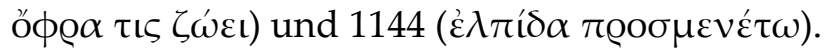

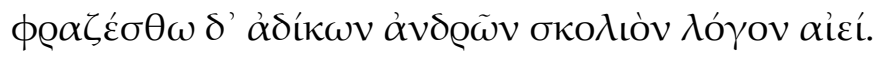

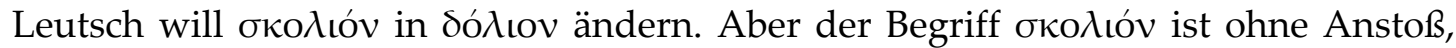

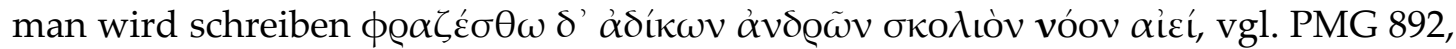

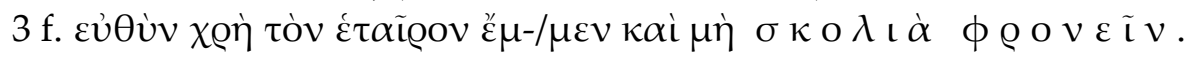

$1163 \mathrm{f}$.

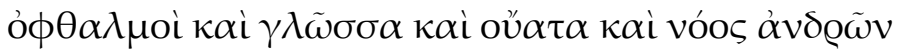

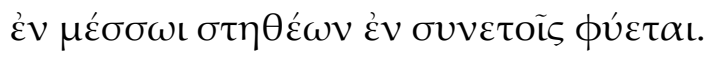

Ein zweiter Praepositionalausdruck mit $\dot{\varepsilon} \nu$ ist nach $\dot{\varepsilon} \nu \mu \varepsilon \dot{\varepsilon} \sigma \sigma \omega \iota \sigma \tau \eta \theta \dot{\varepsilon} \omega \nu$ schon formal störend, und insofern es um die richtige Art der "Wahrnehmung" geht, erscheint der von Geel mit Hilfe des bei Stobaios überlieferten $\varepsilon \dot{v} \xi u ́ v \varepsilon \tau o \varsigma$ hergestellte Dativ

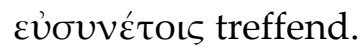

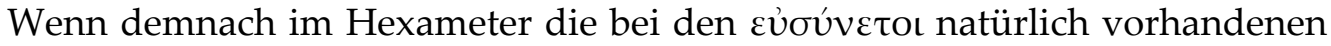
inneren Wahrnehmungsinstanzen aufgezählt werden, muß der völlig allgemeine Genitiv $\alpha \dot{v} \delta \varrho \tilde{\omega} v$ zu vóos in der Tat befremden ${ }^{28}$. Der bei Stobaios überlieferte Singular àvঠ@ós ist nicht besser. Überhaupt paßt der vóos aber nur in den Kreis der aufgezählten Sinnesorgane, wenn er durch ein Attribut als Wahrnehmungsorgan, also als "beobachtender Sinn" gekennzeichnet wird:

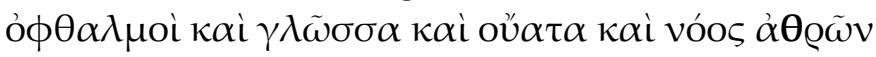

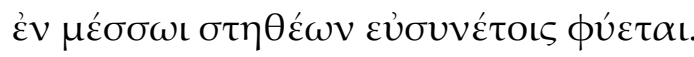

$1165 \mathrm{f}$.

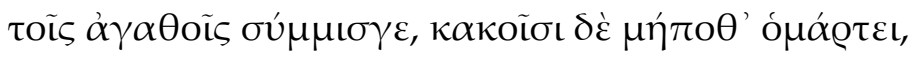

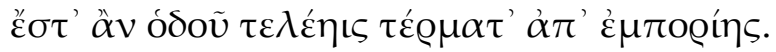

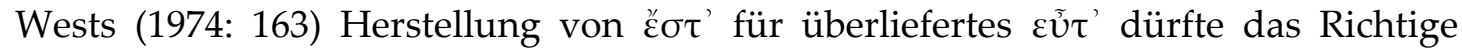
treffen, insofern das Verbot, nicht mit Schlechten zu verkehren, intensiviert wird mit einer zeitlichen Präzisierung von $\mu \eta \dot{\pi} \pi 0$ ' durch ein nachgeschobenes "bis du deinen

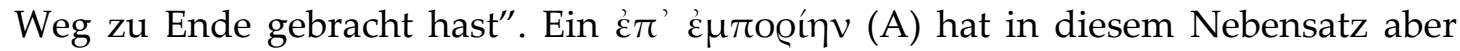
ebensowenig Platz wie das von West aus dem Genitiv $\dot{\varepsilon} \mu \pi$ roón $\rceil$ der übrigen Über-

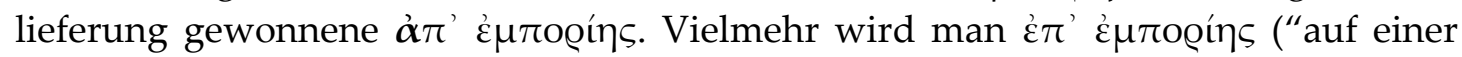
[Handels-]Reise") schreiben und mit den Imperativen des Hauptsatzes verbinden:

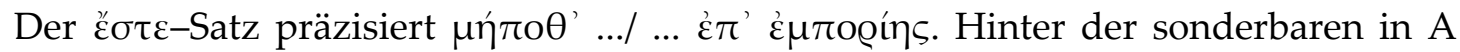
überlieferten Verbalform $\sigma \tau \varepsilon \lambda \varepsilon \eta \tilde{\imath}^{29}$ wird man nicht mehr diagnostizieren als ein

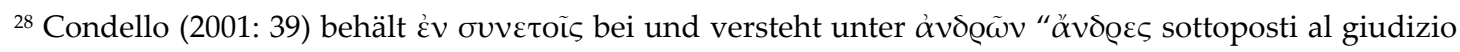

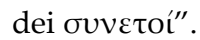

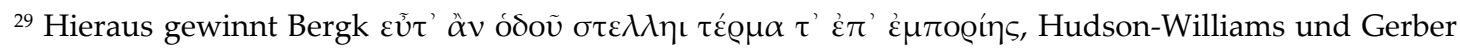

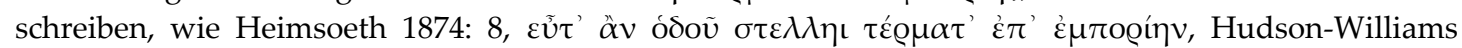


falsch in den Text integriertes interlineares $\sigma$, welches ursprünglich die in dem Satz ("bis du deinen Weg zu Ende gebracht hast”) zu erwartende Aoristform anmahnte:

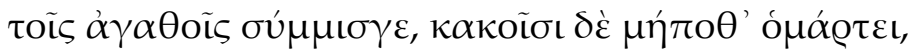

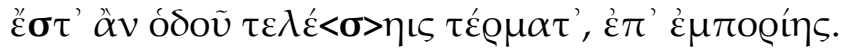

1203-1206

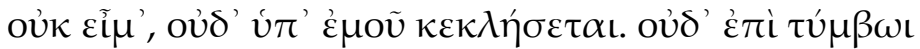

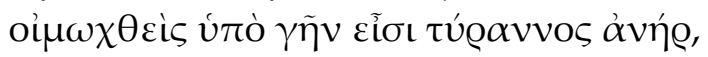

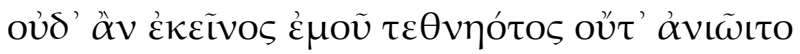

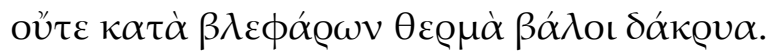

Gerber interpretiert den Einleitungssatz in dem Sinne "I shall not go to his funeral nor will he be invited to me", der sich einem Leser jedoch allenfalls nach Lektüre des weiteren Wortlauts des Epigramms erschließt. Eine praegnante, aber zugleich verständliche Andeutung des im folgenden chiastisch entfalteten Sachverhalts erhält

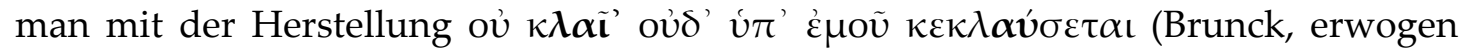
auch bei Hudson-Williams) "er pflegte nicht (um andere) zu weinen, und dafür wird er (bei seinem Tod) auch nicht von mir beweint sein". Zur monosyllabischen Form

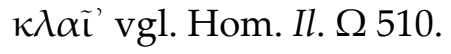

1225 f. (= Stob. IV 22, 5)

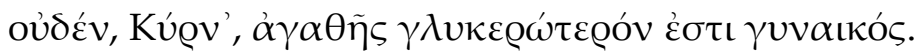

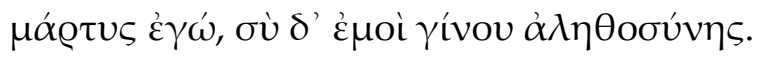

Hudson-Williams' Deutung "I testify to it myself, and you must also do so by taking

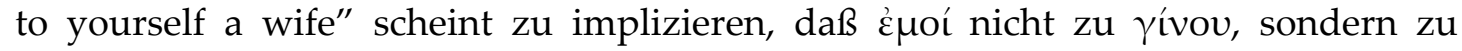

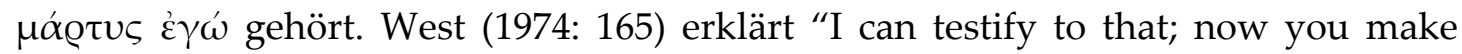
yourself able to testify to my trustworthiness", womit die schlichte Aufforderung "marry one" eine nicht gerade luzide Formulierung erhielte.

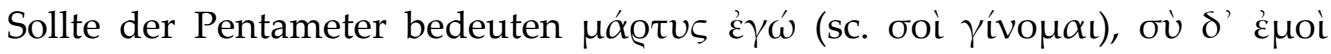

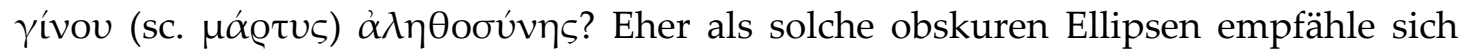

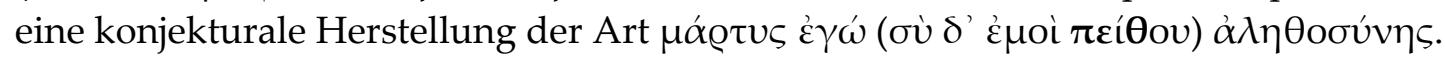

$1249-1252$

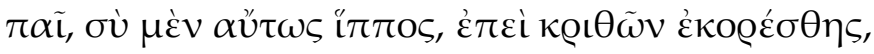

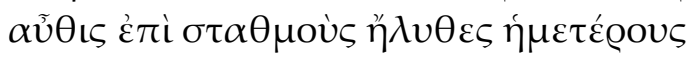

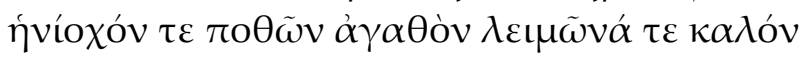

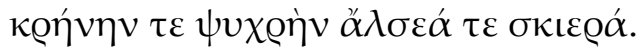

Bergk vermutet hinter dem ersten Hexameter eine Lücke und rekonstruiert den Gedankengang folgendermaßen: "ubi satiatus es, abrumpis vincula et aufugis: post,

übersetzt "when you are going to the end of a journey for business", Gerber "whenever you set out for your journey's goal with a view to trade". 
ubi fames te premit, redis ad pristinum dominum". Dieser Befund ergibt sich fast

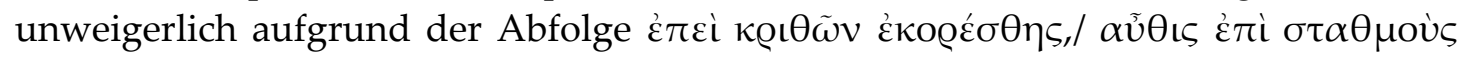

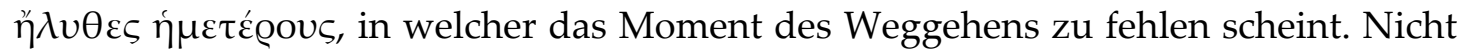
durchschlagend gegen Bergks Diagnose ist die Argumentation Hudson-Williams',

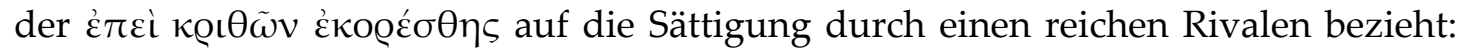
"the horse serves two masters; from one he gets fodder, from the other enjoyment".

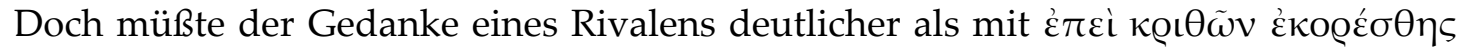

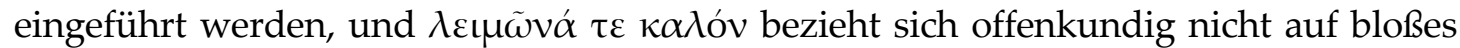
"enjoyment", sondern nimmt die Futter-Metaphorik wieder auf.

Allerdings steckt im ersten Vers, wo $\alpha u ̈ \tau \omega \varsigma$ i $\pi \pi$ o unverständlich ist, noch ein anderer Defekt, den man mit Bergks $\alpha$ $\tau \omega \varsigma$ í $\pi \pi \omega \iota$ (vgl. Anakreon PMG 388, 12 $\gamma v v \alpha \iota \xi i v \alpha u ̛ \tau \omega \varsigma)$ heilen wird. Dann kann man aber den folgenden Temporalsatz in

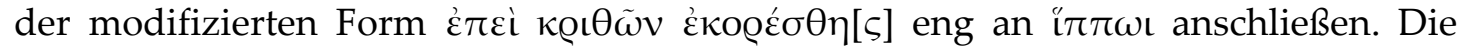
Eigenschaft eines solchen "Pferdes, nachdem es sich einmal (aor.) vom Weizen gesättigt hat," besteht eben darin, an den Ort der Bewirtung immer wieder zurückzukehren. Insofern fügt sich der den Pferde-Vergleich metaphorisierende Ausdruck

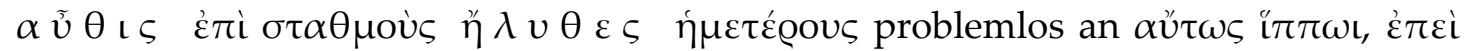

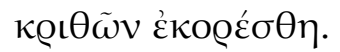

Auch in 1267-1270 bezieht sich das "Vom-Weizen-Gesättigt-Werden" ausschließlich auf das im Gleichnis verwendete Pferd:

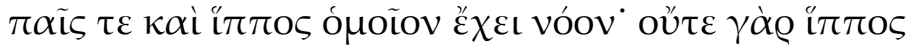

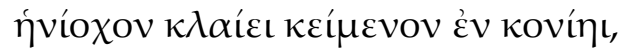

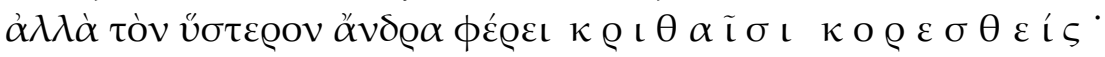

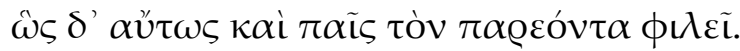

$1259-1262$

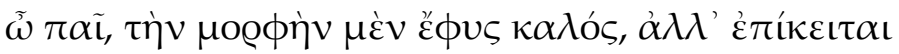

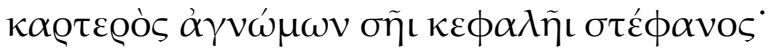

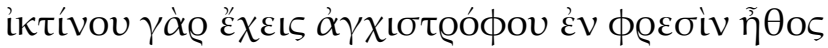

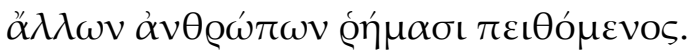

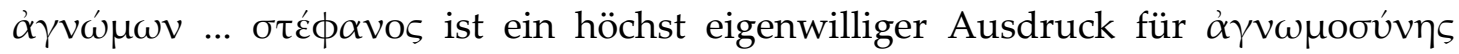

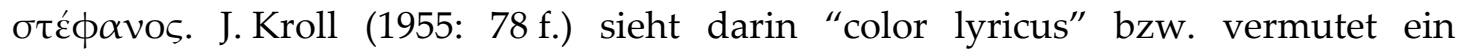
"abgewandeltes lyrisches Vorbild", und Young vergleicht - relativ fernliegend -

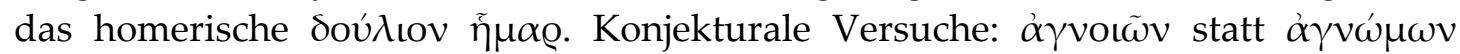
Kukula, $\dot{\alpha} \mu \mu \omega \nu \tilde{\omega} v=\dot{\alpha} v \varepsilon \mu \omega \nu \tilde{\omega} v$ Bergk (verteidigt von van Herwerden 1870: 45),

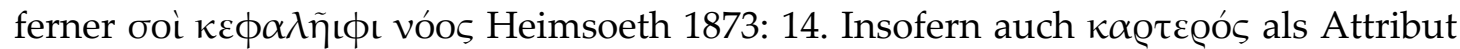

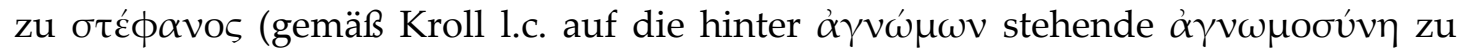
beziehen) höchst problematisch ist, liegt es nahe, den Gedanken des "mit Dummheit bekränzten Beaus" (Kroll) mit einer praegnanten Juxtaposition zum Ausdruck zu bringen:

$$
\ldots \dot{\alpha} \lambda \lambda^{\prime} \dot{\varepsilon} \pi \epsilon^{\prime} \kappa \varepsilon \iota \tau \alpha \iota
$$

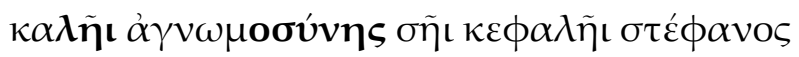

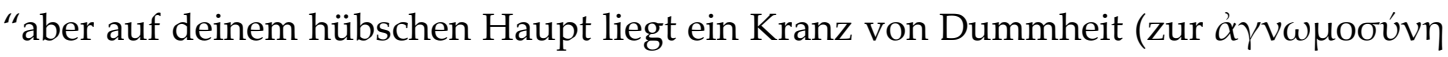
vgl. 896)". Damit dürfte sich Hudson-Williams' Bemerkung, es handele sich bei dem

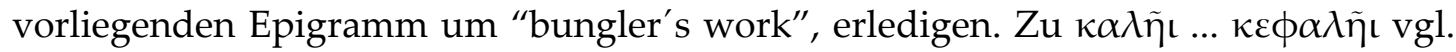




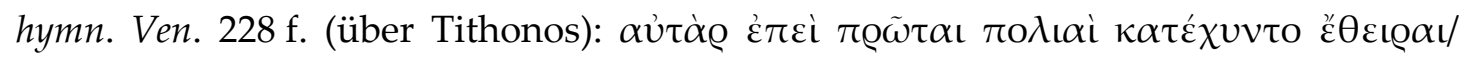

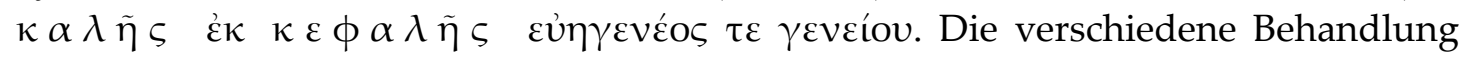
der Quantität der ersten Silbe von $\kappa \alpha \lambda$ ó (grundsätzlich hierzu: Sitzler 1885: 7 f.) in aufeinanderfolgenden Versen wie in $16 \mathrm{f}$. Im selben Vers begegnet diese prosodische Variation bei Kallim. epigr. 29, 3 Pf. Zur Hiatkürzung nach $\kappa \alpha \lambda \tilde{\eta} \iota$ vgl. Hom. Il. B 307

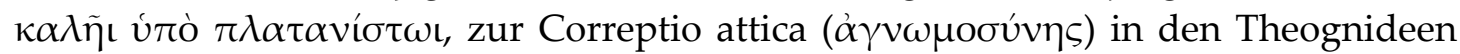
West 1974: 114.

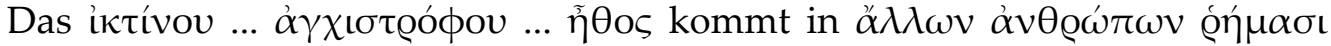
$\pi \varepsilon \iota \theta$ ó $\mu \varepsilon v o \varsigma$ noch nicht richtig heraus, und wenn man mit Bekker gemäß $1152 \alpha \ddot{\lambda} \lambda \omega \omega \nu$ in $\delta \varepsilon \iota \lambda \tilde{\omega} v$ ändert, wird der entscheidende Begriff gerade eliminiert. Eher sollte man das hier überlieferte $\alpha \grave{\alpha} \lambda \lambda \omega \nu$ pronouncieren, etwa durch Abänderung des folgenden

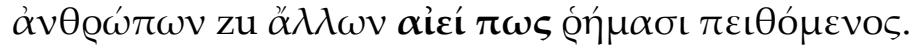

$1353-1356$

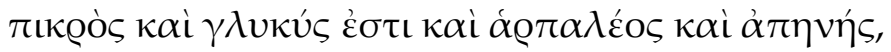

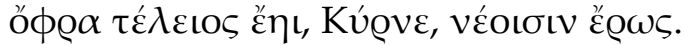

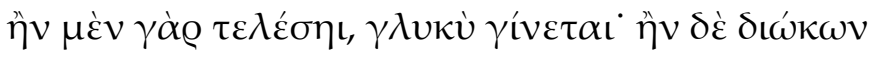

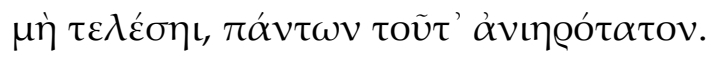

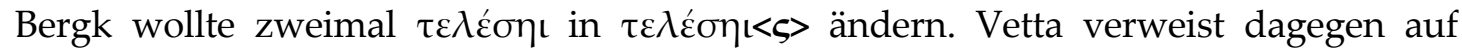
Wackernagel, Vorlesungen über Syntax I 111 ff., wo der archaische Gebrauch der 3. Person Singular im Sinne eines unpersönlichen "man" besprochen wird. Unbestreitbar wird ein solcher Sprachgebrauch entscheidend erleichtert durch das Hinzutreten eines Partizips (vgl. Wackernagel a.a.O. 112 f.), wie es hier im zweiten Glied (

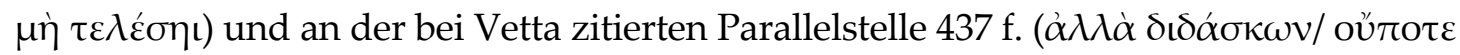

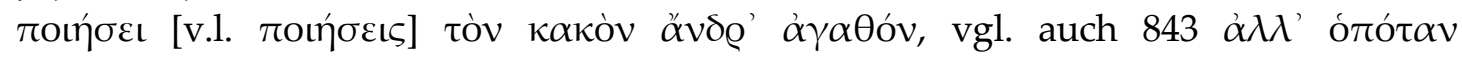

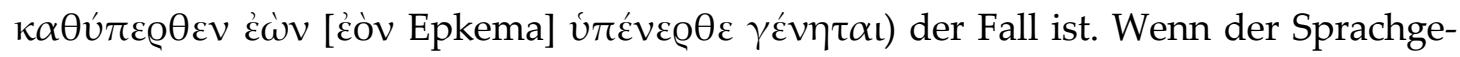
brauch im zweiten Kondizionalsatz derart erleichtert ist (wo übrigens auch das Subjekt

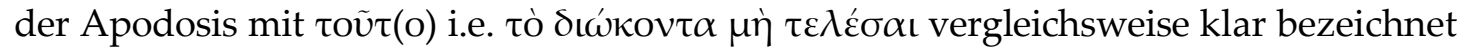

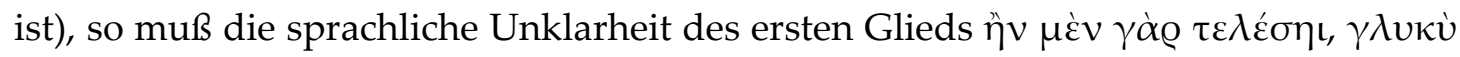

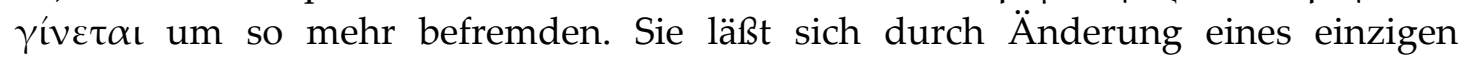

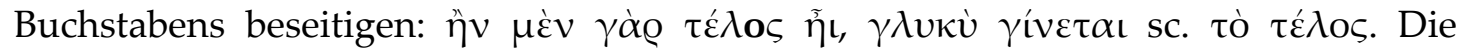
Konjunktivform $\tilde{\eta} \iota$ ist gegenüber $\ddot{\eta} \iota$ in den Theognideen die gewöhnlichere, vgl. West 1974: 107.

$1377-1380$

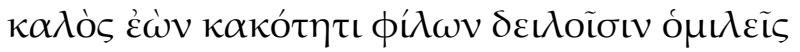

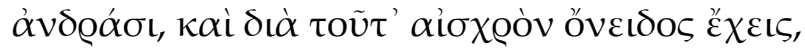

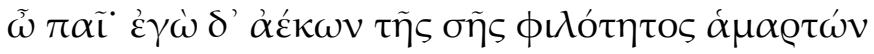

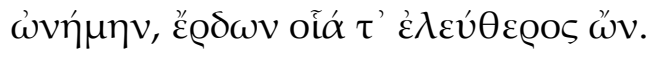

Eine antithetisch von $\kappa \alpha \lambda$ ò $\dot{\varepsilon} \omega \dot{\omega} v$ überleitende abstrakte Praeparation des Gedankens

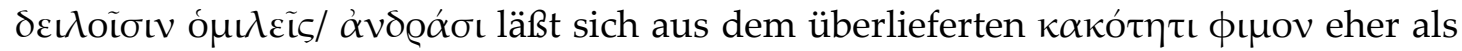

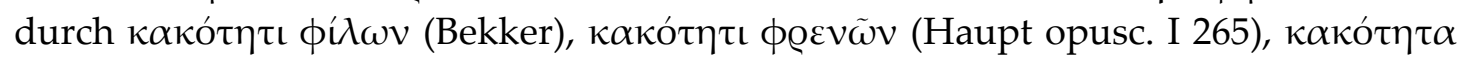

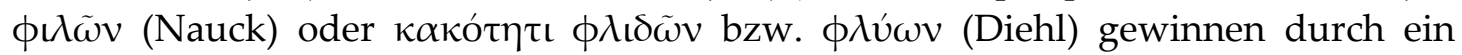




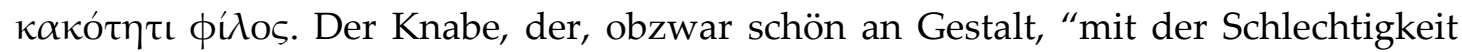
befreundet ist", sucht sich naheliegenderweise auch schlechte Freunde. Ähnlich

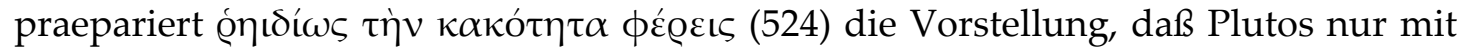
schlechten Menschen verkehrt.

Der Schlußpentameter bleibt zu restituieren durch ein mit $\dot{\varepsilon} \lambda \varepsilon v ́ \theta \varepsilon \rho o \zeta ~ z u$

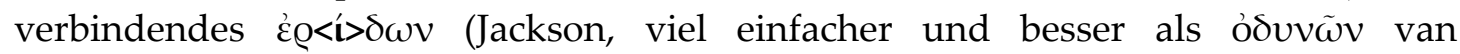
Herwerden 1884: 303).

\section{(15) besonders tiefsitzende Korruptelen}

Mitunter scheint die Korruptel ausgesprochen tief zu sitzen und läßt sich kaum ohne spekulative Ansätze beheben.

$1373 \mathrm{f}$.

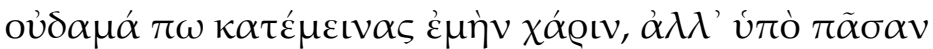

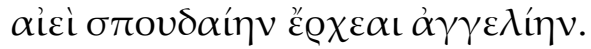

Das den Sprecher Verletzende scheint darin zu liegen, daß der angesprochene Knabe jegliches erotische Anerbieten ( $\dot{\alpha} \gamma \gamma \varepsilon \lambda i ́ \eta v)$ außer demjenigen des Sprechers ernst

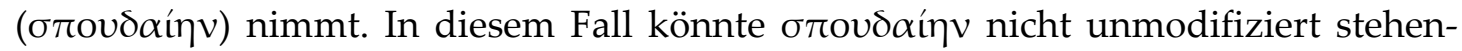
bleiben: van Herwerden (1870: 58) stellte das auf das Handeln des Knaben bezüg-

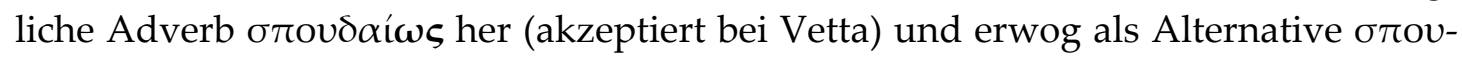
$\delta \alpha ́ \zeta \omega v$, Heimsoeth (1875: 15) schrieb, den Sinn des überlieferten $\sigma \pi 0 v \delta \alpha i ́ \eta v$ um-

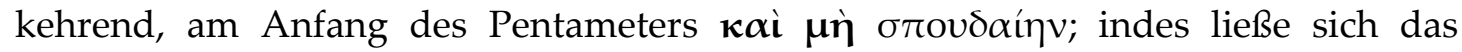
gleiche Ergebnis eines nur vermeintlich ernstgemeinten Anerbietens wesentlich

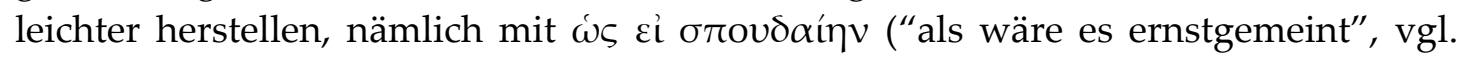
Kuehner/ Gerth II 492). Darüber hinaus suchte Bergk durch seine Konjektur ol' $\chi \varepsilon \alpha$ เ

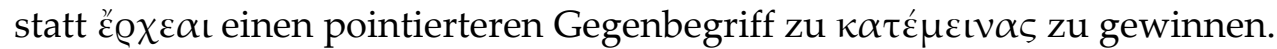

Auf das Ganze betrachtet, erscheint jedoch eine Rekonstruktion in folgender Richtung erfolgversprechender:

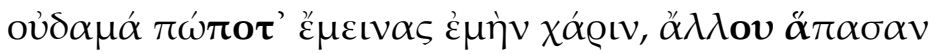

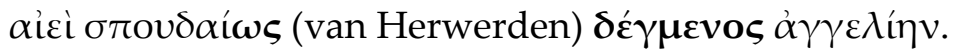

"Nie hast du jemals meine Gunstbezeugung abgewartet, während du von einem anderen jede beliebige (Liebes-)Botschaft stets voller Eifer entgegennahmst". Ein

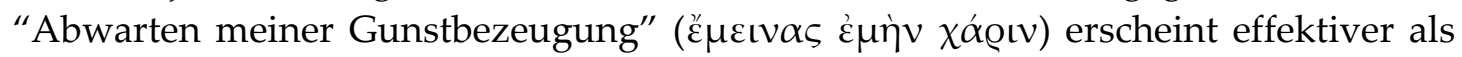
Gegensatz zum zweiten Glied gegenüber einem "meinetwegen Ausharren"

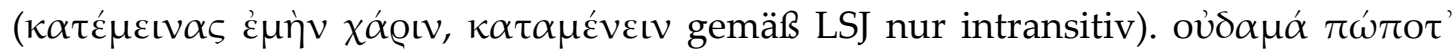

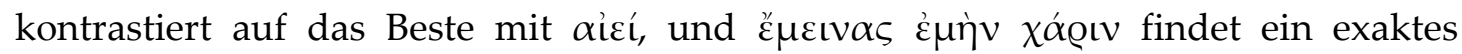

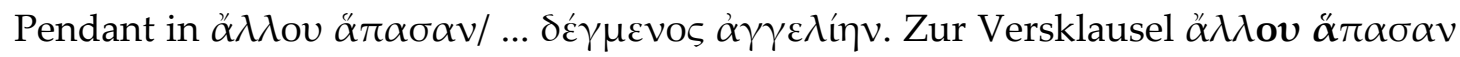

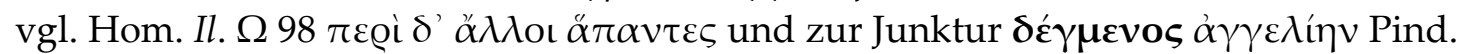
Pyth. 2, 41 und Eur. IT $447 \mathrm{f}$.

Besonderes Augenmerk verlangt das innerhalb der Theognideen aus sprachlichen Gründen modernistisch anmutende lange Gedicht 903 ff. über das rechte Verhältnis zum Geld: 
$903 \mathrm{f}$.

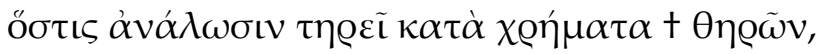

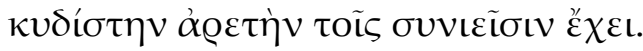

Cataudella (1956: $40 \mathrm{f.}$ ) schreibt $\theta \eta \dot{\sigma \omega \nu}$. Eine paläographisch weniger elegante Möglichkeit, die jedoch den Vorzug hätte, ein zu $923 \mathrm{f}$. (oư $\tau \omega, \Delta \eta \mu o ́ \kappa \lambda \varepsilon เ \varsigma, ~ \kappa \alpha \tau \dot{\alpha}$

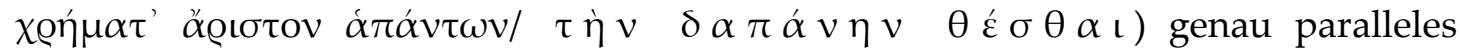

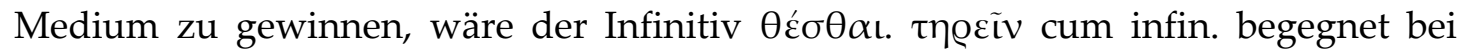

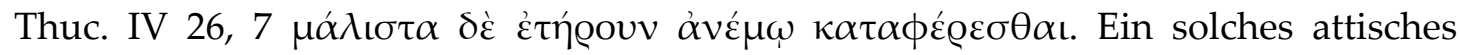
Idiom würde gut passen zu einem Gedicht, dessen offenbar relativ junges Datum häufiger hervorgehoben wurde.

Erbses Umkehrung der Verteilung zwischen Hauptsatz und Partizip durch

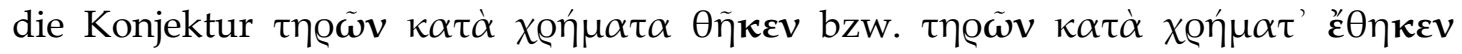
(1998: 240 f.) ist kaum erforderlich.

911-914

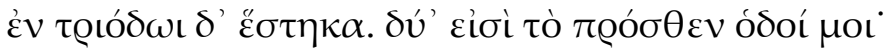

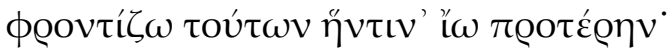

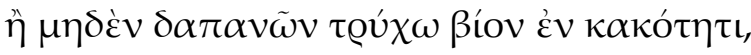

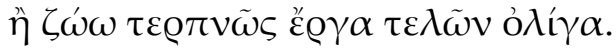

$\tau \varepsilon \lambda \tilde{\omega} \nu$ ò $\lambda \hat{i} \gamma \alpha$ ("accomplishing little", Gerber) ist kaum richtig in einem Zusammenhang, wo es nicht um Leistungen, sondern nur um materiellen Besitz gibt. Die Antithese zu $\mu \eta \delta \dot{\varepsilon} v \delta \alpha \pi \alpha \nu \tilde{\omega} v$ erfordert etwas der Art "wenig Besitz zurückbehaltend", $\chi \varrho \eta \dot{\mu} \mu \alpha \tau^{\prime}$ '̌ $\chi \omega \nu$ ò $\lambda \hat{\gamma} \gamma \alpha$.

915-920

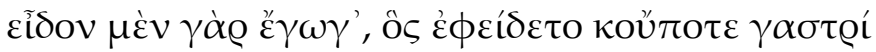

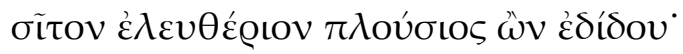

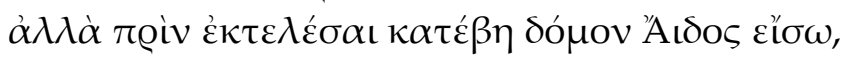

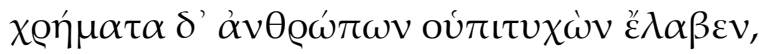

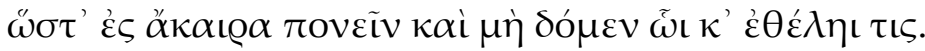

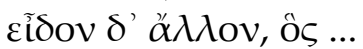

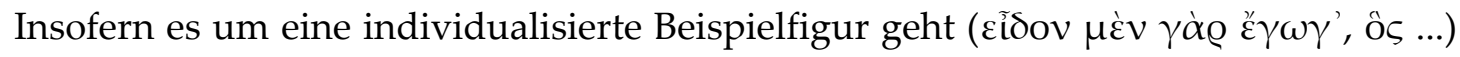

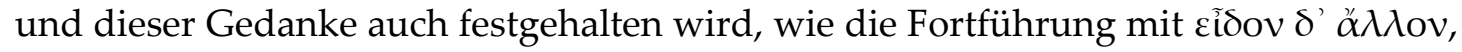

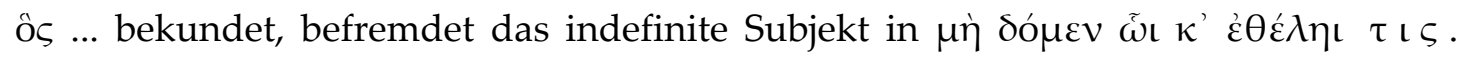

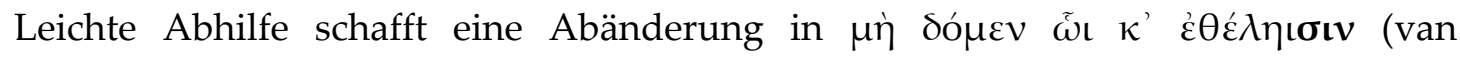
Herwerden 1870: 53 : $\tilde{\omega} \iota \kappa \varepsilon$ $\theta \dot{\varepsilon} \lambda \eta \iota \sigma \iota v$ Wilamowitz gemäß Youngs Apparat). Ebendiese Konjunktivform ist am Hexameterschluß belegt in 139 oủdé $\tau \omega \iota \dot{\alpha} v \theta \varrho \omega ́ \pi \omega \nu$ $\tau \alpha \varrho \alpha \gamma^{\prime} \operatorname{v\varepsilon \tau } \alpha \iota$, ö $\sigma \sigma^{\prime} \dot{\varepsilon} \theta \dot{\varepsilon} \lambda \eta \iota \sigma \iota v$.

923-930

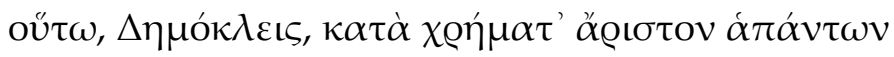

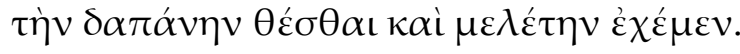




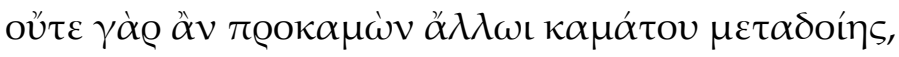

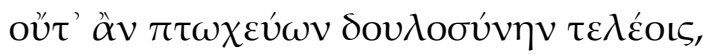

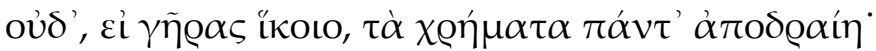

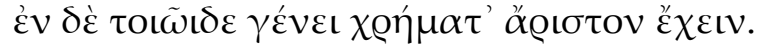

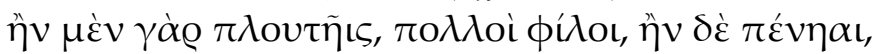

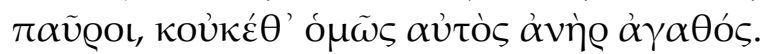

In Vers 925 bietet die Hauptüberlieferung, auch Pap. Berol. 2122030, кá $\mu \alpha \tau o v$. $\kappa \alpha \mu \alpha ́$ tov findet sich nur in einem cod. recentior, und West erwägt als Alternative im Apparat den Plural $\kappa \alpha \mu \alpha \dot{\tau} \tau \omega v$. Insofern aber derjenige, der nach einem arbeitsreichen Leben zu früh stirbt, seinen Erben nicht nur "Anteil gibt" an seinem Besitz, also am Ergebnis seiner Mühen, sondern diese im Gegenteil vollständig übergibt, scheint

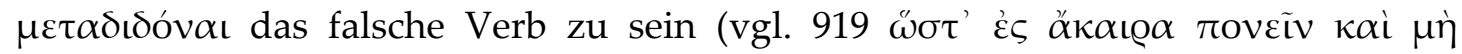
$\delta$ ó $\mu \varepsilon v \quad \tilde{\omega} \kappa^{\prime} \dot{\varepsilon} \theta \dot{\varepsilon} \lambda \eta \iota \sigma \iota$, zum Text z.St.); Bergk erwägt u.a. $\pi \alpha \varrho \alpha \delta o i ́ n \varsigma . ~ D a r a n$ ändert auch nichts die bei Hudson-Williams von "give a share of" differenzierte Sonderbedeutung "give as a share", weil eben der Gedanke eines "Anteils" überhaupt nicht vorschwebt. Eine prägnante Antithese zu тоок $\alpha \mu \omega \nu$ (zeitlich, kaum im

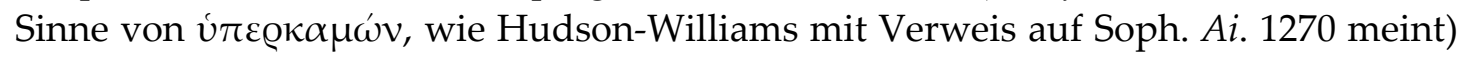

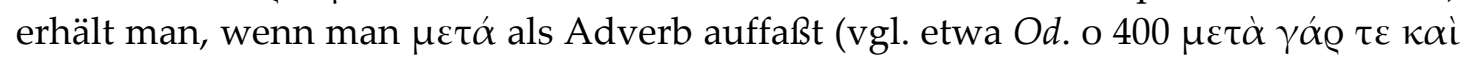

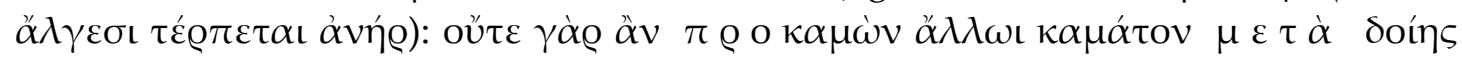
"weder wirst du, nachdem du dich vorher gemüht hast, die Frucht deiner Mühen $\mathrm{n}$ a ch h e r einem anderen geben".

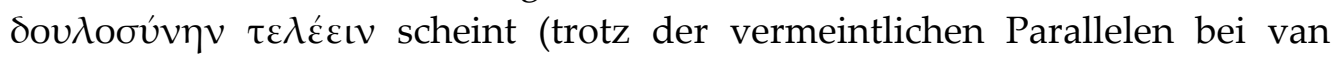
Groningen) der falsche Ausdruck für "endure slavery" (Gerber). Obwohl $\tau \varepsilon \lambda$ ćoı durch Pap. Berol. 21220 bestätigt wird, erscheint das Verb zunächst fragwürdig, wie

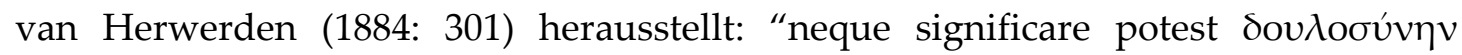
$\tau \varepsilon \lambda \varepsilon_{\varepsilon} \varepsilon v$ in fine vitae servitutem experiri, neque satis probabiliter interpretari licet: neque mendicando servitutem efficias, quoniam ipsa mendacitas est servitus, cuius causa potius prodiga vita praedicanda fuit". Ein idiomatischer epischer Ausdruck für "Unangenehmes erdulden", der insbesondere von das Leben beendenden Umständen

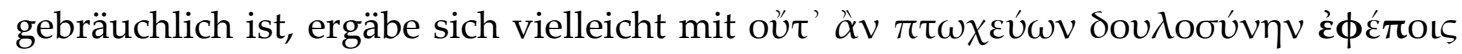

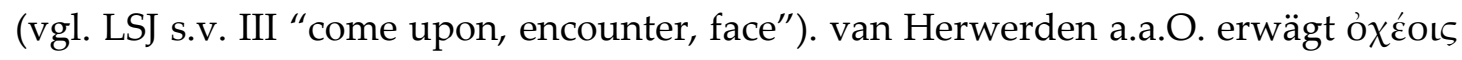
und $\tau \alpha \lambda \alpha ́ \sigma \alpha ı \varsigma$ (letzteres auch Bergk), entscheidet sich am Ende aber für ỏ $\tau \lambda \dot{\varepsilon}$ oıs, ein

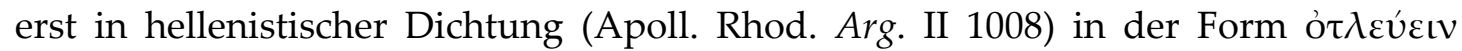

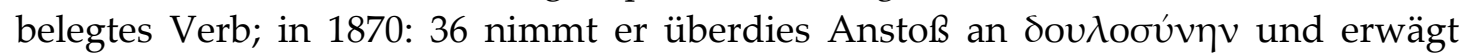

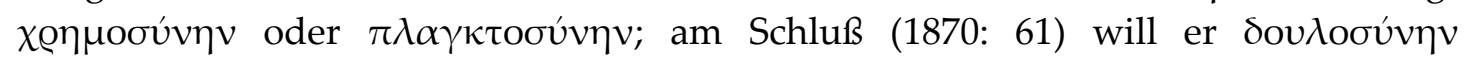

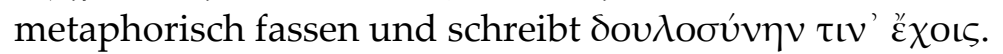

Besser als konjekturale Versuche an $\tau \varepsilon \lambda \dot{c}$ oı $\varsigma$ vorzunehmen, erscheint es aber,

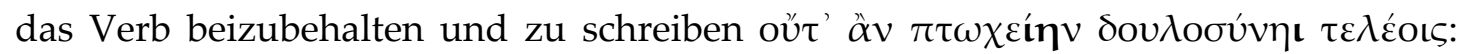
Das bereits in Vers 922 als Folge der Armut herausgestellte $\pi \tau \omega \chi \varepsilon v ́ \varepsilon เ v$ kulminiert im schlimmsten Fall im Verlust der Freiheit. Dann würden in dem Distichon 925 f. die Folgen der beiden verkehrten Verhaltensweisen in paralleler Weise jeweils in zwei Stufen dargestellt, die durch die Hauptzäsur des jeweiligen Verses gesondert werden: Exzessives Sparen führt zunächst zu großen Mühen ( $\pi$ ○ o $\kappa \alpha \mu \omega \nu)$ und dann zu einer unfreiwilligen Übergabe des Reichtums an einen dritten (ä $\lambda \lambda \omega \iota \kappa \alpha \mu \alpha ́ \alpha \tau$

${ }^{30}$ Vgl. Kotansky 1993: 1-5. 


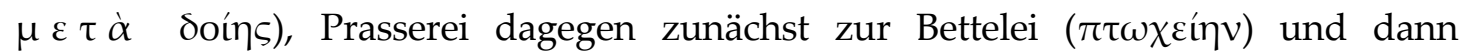

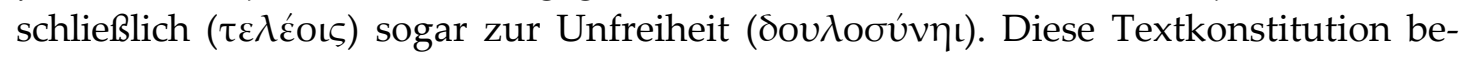
deutet freilich immer noch ein Abweichen vom ältesten Textzeugen, dem Papyrus,

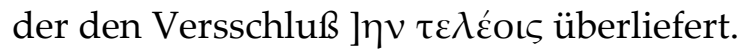

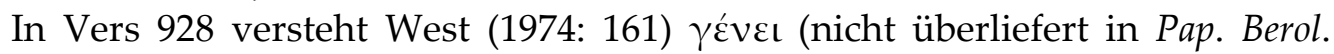
21220) in dem Sinne "class of people, sc. the elderly" (diesen Sprachgebrauch empfand Bergk als "insolenter dictum" und erwog $\pi \varepsilon \dot{\varepsilon} \lambda \varepsilon$ ); Hudson-Williams deutet "in this age or society of ours" bzw. "among such a generation of citizens as the present"; in seiner Appendix (1910: 261) wird die sprachlich bedenkliche Alternative "on this principle" erwogen. Eine Aussage "unter alten Leuten (oder: in der heutigen Gesellschaft) ist es am besten Geld zu haben" würde jedoch schlecht in das vorliegende Gedicht passen, insofern darin deutlich impliziert wäre, daß es eben doch sicherer ist zu sparen, also die Position der abwägenden Mittelhaltung eindeutig verlassen würde. Eher als eine solch konkrete Stellungnahme zugunsten des Sparens erwartet man eine abstrakte Aussage der Art "Zu solchem Anteil/ In solcher Proportion ist es am besten Geld zu haben", mit einem Rückverweis auf die oü $\tau \varepsilon-$

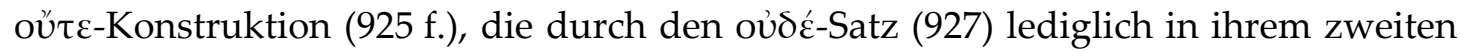
Glied erweitert wird (realisiert in der oben gegebenen Interpunktion): $\dot{\varepsilon} \nu \delta \dot{\varepsilon} \tau o เ \tilde{\omega} \iota \delta \varepsilon$

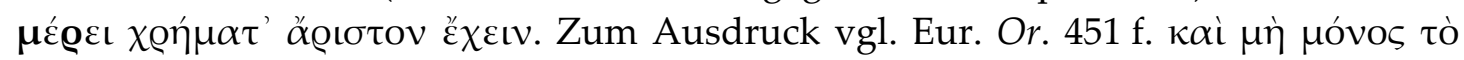

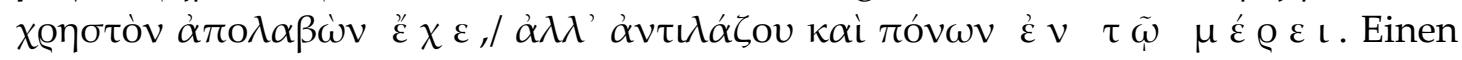
gedanklich noch wesentlich praeziseren Ausdruck, der zugleich die unmittelbar zuvor genannten Extreme miteinbezieht, erhält man aber, wenn man mit etwas stärkerem Eingriff schreibt

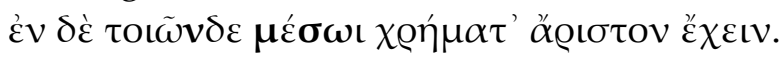

Vgl. 837-840

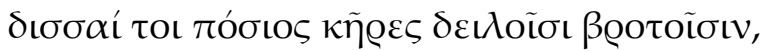

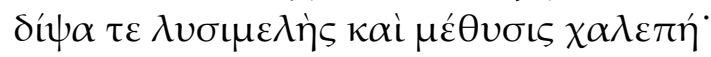

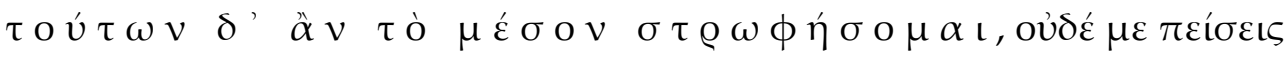

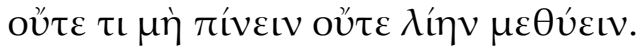

Mit diesem Begriff des $\mu \varepsilon ́ \sigma o v$ vor Augen kann man auch die nach dem praezisen

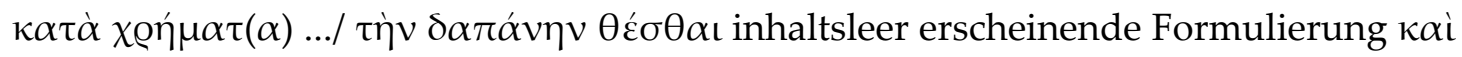

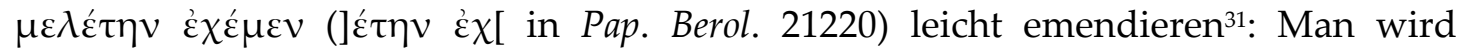

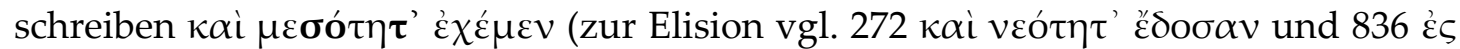
$\kappa \alpha \kappa o ́ \tau \eta \tau$ ' ع́ $\beta \alpha \lambda \varepsilon v)$ "und die Mitte bewahren", so daß der Rekurs auf die "Mitte" negative Ausführung der beiden Extreme rahmt. Das philosophisch-abstrakte $\mu \varepsilon \sigma o ́ \tau \eta \varsigma$ paßt exakt in die innerhalb der Theognideen singuläre, modernistisch wirkende Diktion des vorliegenden Gedichts. Die als Similie behandelte Hesiod-Stelle op. 457

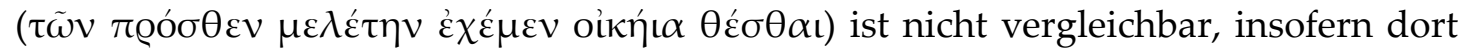
sowohl durch Genitiv als auch durch epexegetischen Infinitiv genau bezeichnet ist, w o r u m Sorge getragen werden soll.

Diese Anweisung wird im Schlußdistichon durch eine Explikation der schäd-

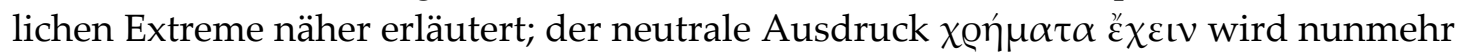

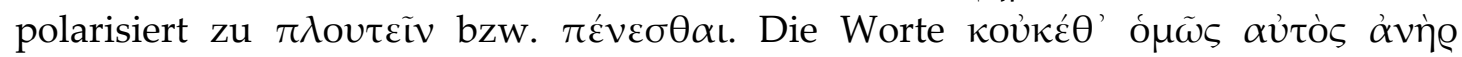
$\grave{\alpha} \gamma \alpha \theta$ ó $\varsigma$ ("and you are no longer the same worthy man you once were", Gerber)

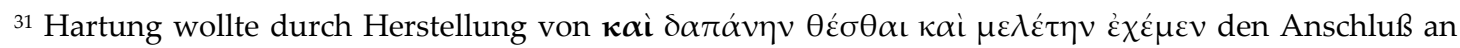
das Vorige notdürftig bessern.
} 
ergeben freilich eine höchst sonderbare Aussage, zumal die Eigenschaft $\alpha \gamma \gamma \alpha \theta$ ó (nicht überliefert in Pap. Berol. 21220) durch die Finanzlage gerade nicht affiziert wird und ein "du giltst als" nicht eben leicht als Auxiliarverb suppliert wird. Man erwartet: "und du wirst, obwohl der gleiche Mann, nicht mehr gleichermaßen sozial

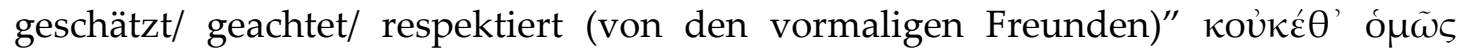

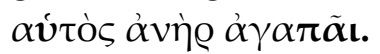

Im ganzen:

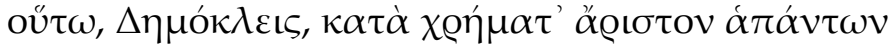

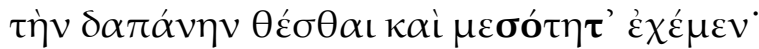

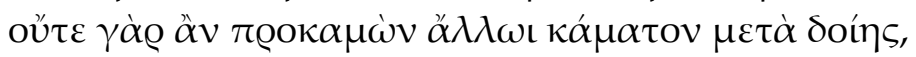

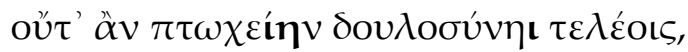

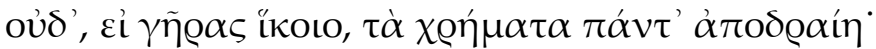

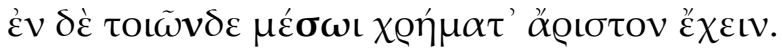

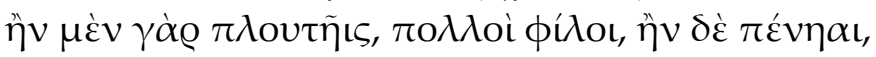

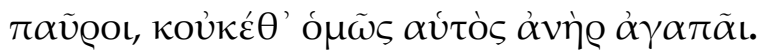

\section{(16) Zwei mythische Paradeigmata}

Von besonders schweren Korruptelen heimgesucht sind ferner zwei mythische Paradeigmata.

$1123-1128$

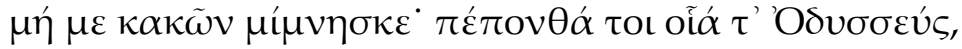

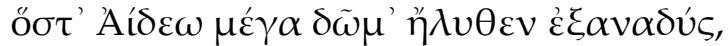

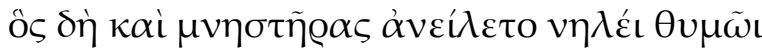

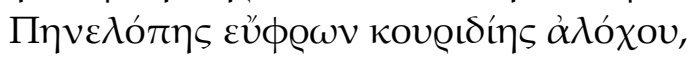

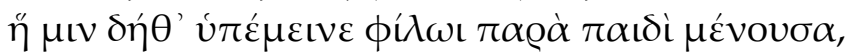

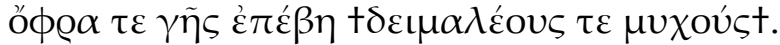

Insofern das Tertium comparationis zwischen dem Sprecher und Odysseus das überstandene Leiden (perf. $\pi \varepsilon ́ \pi \operatorname{ov} \theta \alpha$ ) ist, kann es in Vers 2 nicht um den Gang des Odysseus in die Unterwelt gehen (Aí $\delta \varepsilon \omega \mu \varepsilon \tau \dot{\alpha} \delta \tilde{\omega} \mu$ ' $\eta \hat{} \lambda v \theta \varepsilon v$ nach Sitzlers Konjektur), sondern nur um seine schließliche Heimkehr nach überstandenem Abenteuer. Falls man nicht die seltene Verbindung von $\dot{\varepsilon} \xi \alpha v \alpha \delta v ́ \varepsilon \sigma \theta \alpha \iota$ mit Akkusativ akzeptieren will (die LSJ nur mit einem pythischen Orakel bei Pausan. IV 12, 4

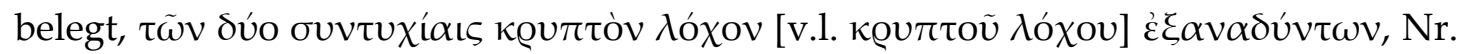

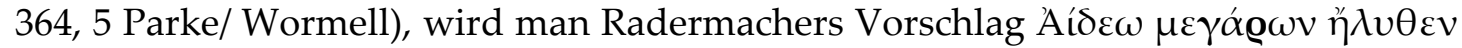

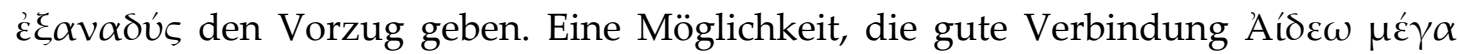

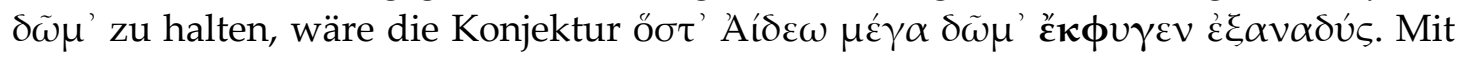

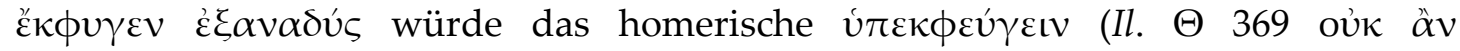

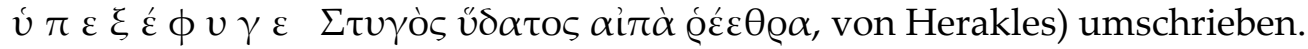

Weitere Korruptelen verbleiben in den folgenden Distichen. Wie sich zu dem

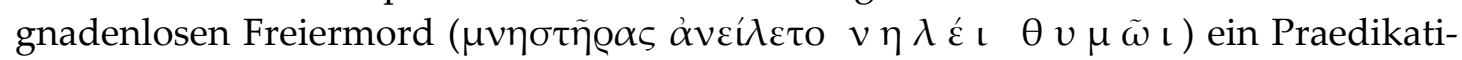
vum der Bedeutung "heiter" ( $\varepsilon$ steht es mit der Variante "verständig" ( $\left.\mu^{\prime} \phi \varrho \omega v\right)$; Hudson-Williams erwägt dieses

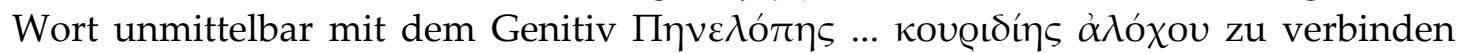


(wohl in der Bedeutung "eingedenk"), sagt dann aber zurecht: "ع́ $\mu \phi \varrho \omega \nu$ would be more applicable to Od. in a distant land before his return". Stattdessen erwartet man ein Partizip, welches das persistierende Verlangen des Odysseus nach seiner Gattin

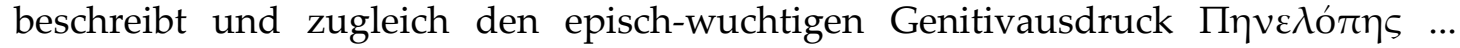

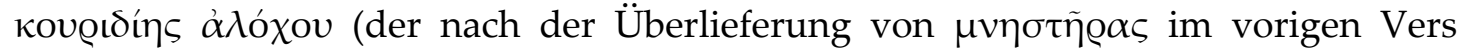

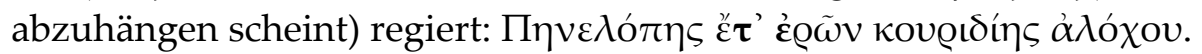

Mit dem andauernden Verlangen des Odysseus scheint im folgenden Distichon die langjährige Liebe der Penelope zu kontrastieren: $\eta \mu^{\mu} \nu \delta \eta \theta^{\prime} \quad \dot{\tau} \pi \varepsilon \dot{\mu} \mu \varepsilon \imath \varepsilon$ (imperf.). Erwägen könnte man hier auf den ersten Blick eine Abänderung in $\eta ๊ \mu \nu$ $\delta \eta \dot{v} v \dot{\tau} \varepsilon \dot{\mu} \mu \varepsilon v \varepsilon$, insofern temporales $\delta \eta \dot{v}$ (anders als das nur vereinzelt in der Ilias begegnende $\delta \eta \theta \alpha \dot{\alpha}, \mathrm{E} 587$ und K 52) in der Odyssee formelhaft gebraucht wird von

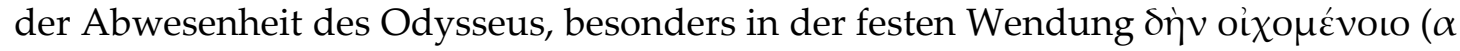

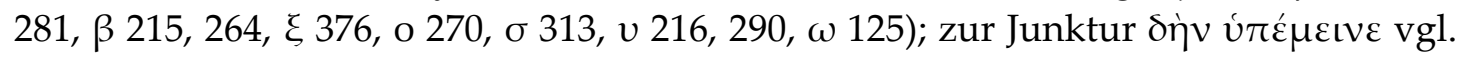

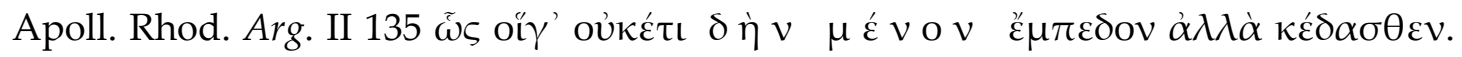
Diese homerische temporale Verwendung von $\delta \eta ́ v$ könnte sich (zumal in einem homerischen Kontext) dann problemlos fügen neben die lokale Bedeutung "weit entfernt", die P. von der Mühll (1932: 135-138 und 1955: 112) überzeugend für zwei Stellen der Theognideen (494 und 597) wahrscheinlich gemacht hat.

Aber bei genauerer Betrachtung scheint das Problem dieses Verses tiefer zu

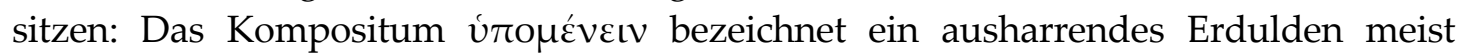
unangenehmer Umstände, ist nicht belegt im Zusammenhang eines sehnsuchtsvollen Erwarten eines Vermißten und würde ein Mißverständnis in dem Sinne "die ihn lange erdulden mußte" nahelegen. Auch ein sich anbietendes $\dot{\pi} \pi \varepsilon \dot{\mu} \mu \nu \varepsilon$ brächte keine Besserung; transitives $\dot{\pi} \pi \mu \varepsilon ́ v \varepsilon \iota v$ wird vorzugsweise gebraucht von Dingen, die einer bestimmten Behandlung "harren”. Vor allem aber ist die Wiederholung $v i \varepsilon \dot{\mu} \mu \varepsilon v \varepsilon$... $\mu \varepsilon \dot{v}{ }^{\prime} v \sigma \alpha$ in den Theognideen beispiellos. Nach allem Gesagten kann die Korruptel nur in $\delta \eta \theta^{\prime} \dot{v} \pi \varepsilon \dot{\mu} \mu \varepsilon v \varepsilon$ liegen, zumal der Versschluß gesichert ist durch Hom. Od. $\lambda$

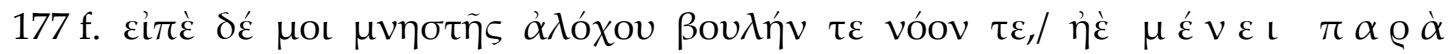

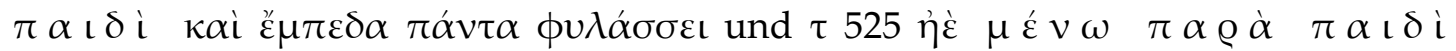

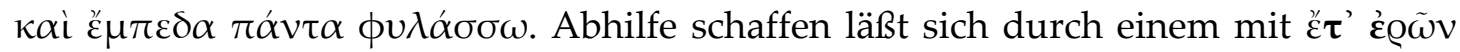

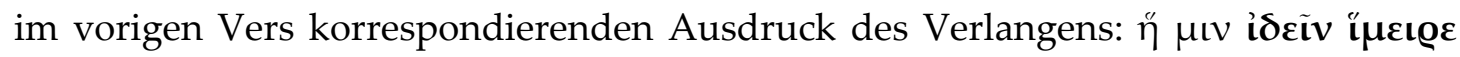

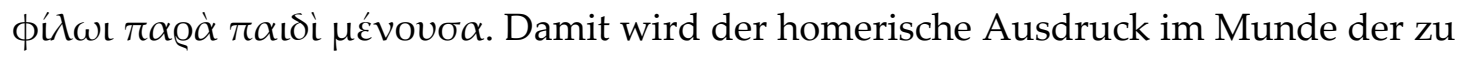
Odysseus sprechenden Kalypso perspektivisch umgekehrt und auf Penelope übertragen (Od. \& 208-210):

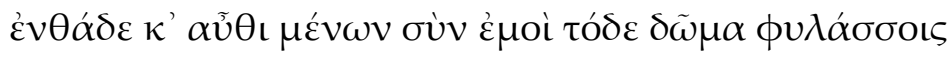

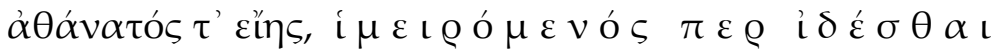

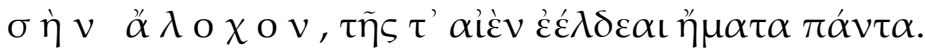

Aktives ífé́@єıv mit Infinitiv ist zwar unhomerisch, aber für die Elegie durch Solon fr. 13, 7 gesichert (vgl. auch Hesiod fr. 199, 2 Merkelbach/ West).

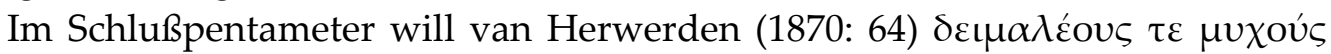

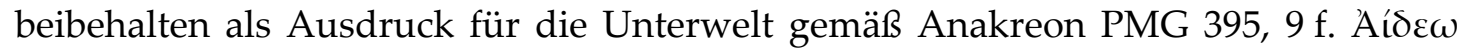

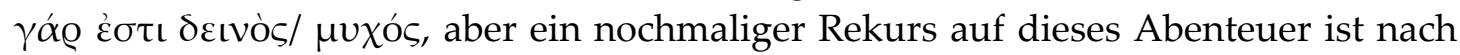
1124 höchst unwahrscheinlich. Haupt opusc. I 265 schrieb (in Wests Apparat

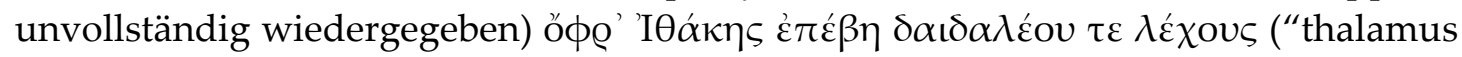
eleganter et affabre structus"), nahm also einen auf die Rückkehr des Odysseus bezüglichen "bis"-Satz an. Dagegen versuchte Sitzler mit sehr viel geringerem 


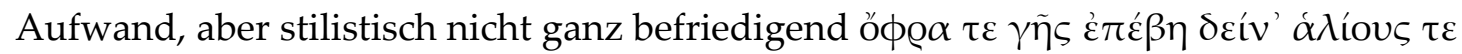

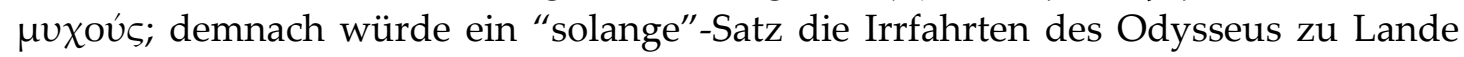
und zu Wasser bezeichnen. Dieser Gedanke wäre sehr angemessen, freilich müßte man

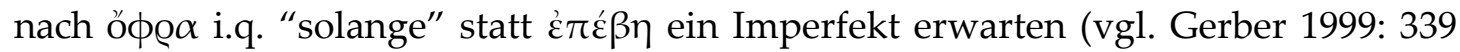

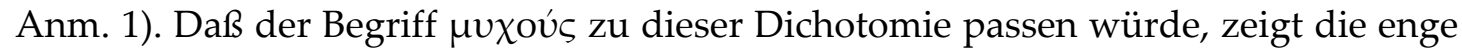

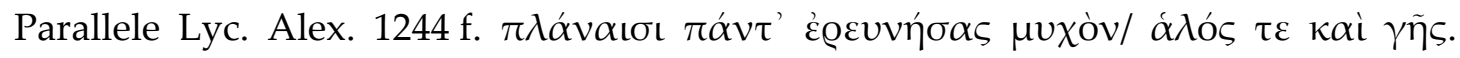
Demnach wird man den Schlußpentameter (hinter welchem Hudson-Williams kaum $\mathrm{zu}$ Recht den Ausfall weiterer Verse annimmt) am leichtesten sanieren mit einem

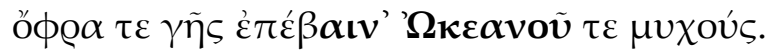

Das Gedicht ist nach der hier gegebenen Rekonstruktion wahrscheinlich erotischer Art: Der mit $\mu$ í $\mu \nu\rceil \kappa \varepsilon$ angesprochene Adressat dürfte ein Knabe sein, der an die Mühen erinnert werden soll, die der Sprecher während einer längeren Abwesenheit erduldet hat, um schließlich zu dem geliebten Knaben zurückzukehren. Die Personen des Mythos sind Paradigmen für eine glückliche Weiterführung des

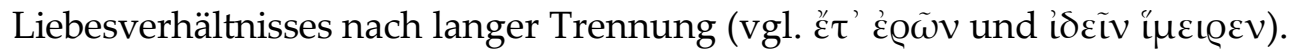

Im ganzen:

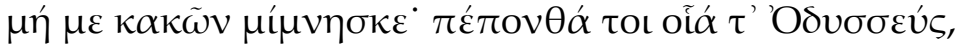

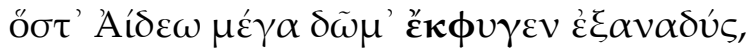

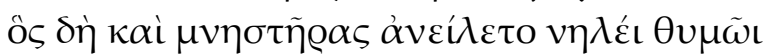

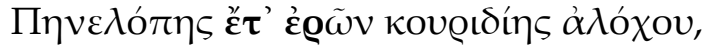

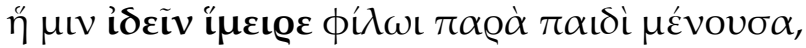

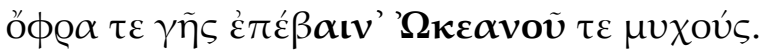

$1283-1294$

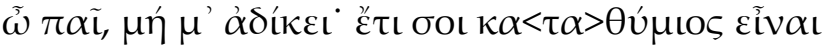

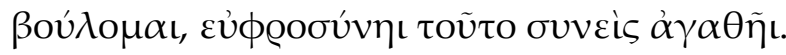

1285

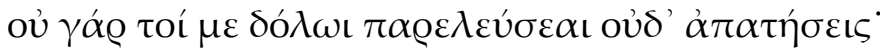

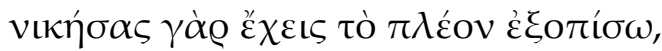

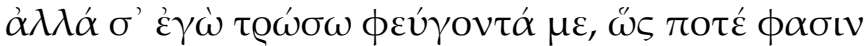

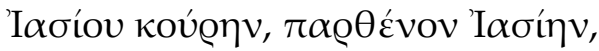

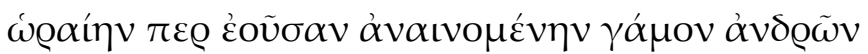

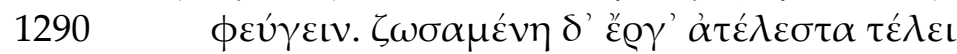

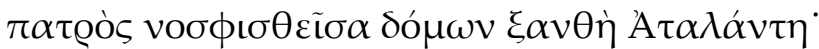

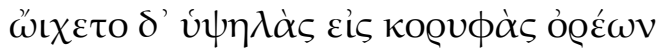

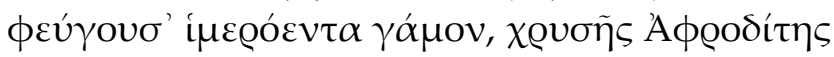

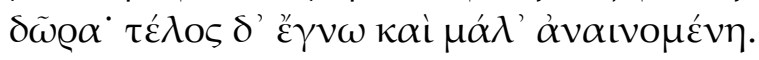

West (1974: 165-167; vgl. bereits Hudson-Williams zu 1283-1294), gibt eine recht komplizierte analytische Deutung des Gedichts, wonach eine Urfassung (in welcher Atalante als eine ihre Konkurrenten tötende Läuferin mit dem Sprecher verglichen wird, 1283-1288 a) und eine sekundäre Erweiterung (in der Atalante unter dem Gesichtspunkt der Flucht mit dem Knaben verglichen wird, 1288 b-1294) ineinander geflossen wären. C. Carey (1984: 362-366, bes. 365 f.) schließt sich dieser Deutung im Prinzip an, sieht hinter den verschiedenen Ausprägungen des Atalante-Vergleichs aber nicht wie West zwei verschiedene Dichter wirksam, sondern erklärt die 
Verschiebung aus den von Hermann Fränkel untersuchten Eigenheiten des archaischen Gleichnisses. Grundsätzlich gegen eine analytische Betrachtungsweise: Renehan 1983: 24-27, Koniaris 1984: 104-106; ferner Gerber 1999: 371 n. 4. Weitere Doxographie bei Gerber 1991: $213 \mathrm{f}$.

Demgegenüber wird hier eine in sich konsistente Urfassung angenommen, die Atalante von vorneherein unter dem Gesichtspunkt zum Scheitern verurteilter Flucht heranzieht. Vorgeschlagen sei etwa folgender Wortlaut:

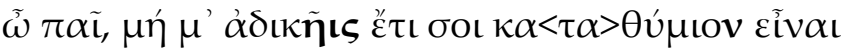

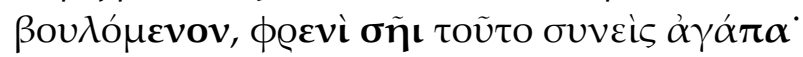

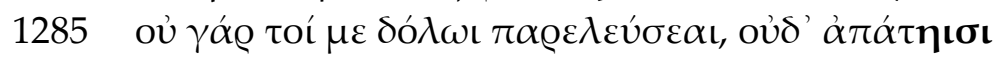

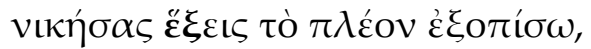

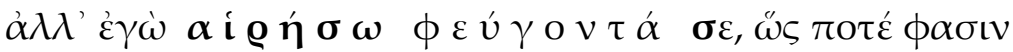

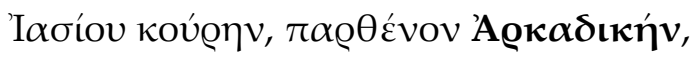

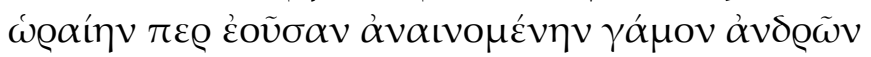

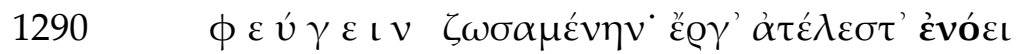

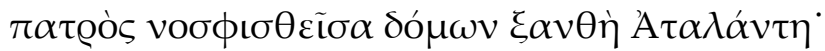

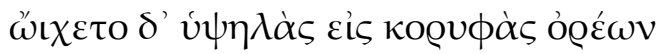

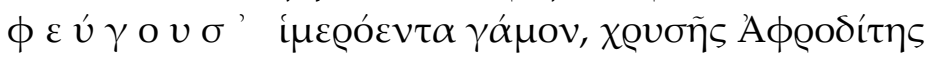

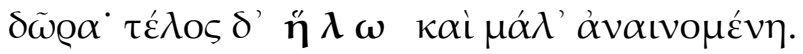

$\mathrm{Zu}$ den einzelnen Problemen:

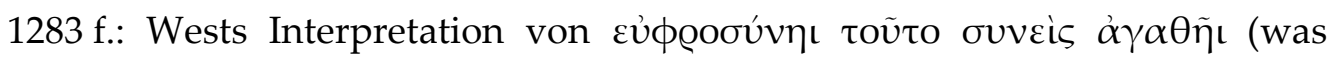

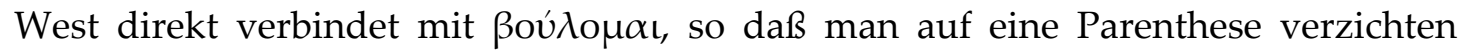

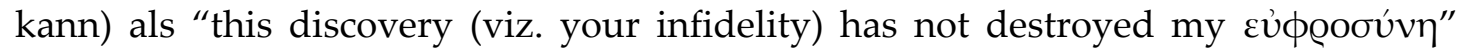
(1974: 165) ist unwahrscheinlich, weil ovvı́́vaı ein Begreifen, nicht ein bloßes ZurKenntnis-Nehmen bezeichnen sollte. Gegen Wests Deutung des Eingangsdistichons vgl. auch Carey 1984: $362 \mathrm{f}$.

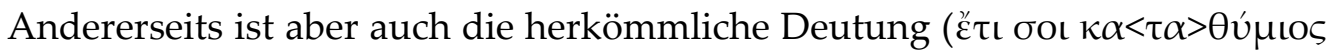

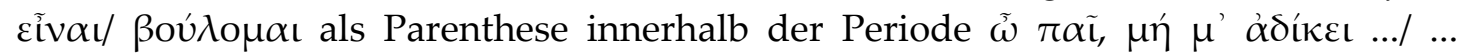

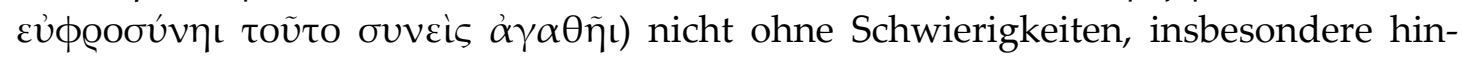

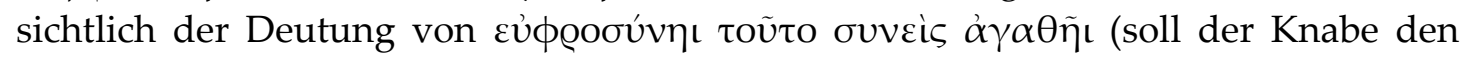
ihm von Sprecher erteilten Rat mit "gelassener Heiterkeit" zur Kenntnis nehmen und rationalisieren?).

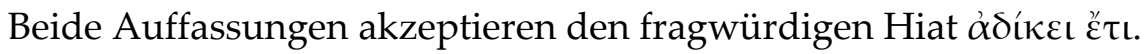

Die oben hergestellte Textfassung bedeutet: "Knabe, damit du mir, der ich dir zu Gefallen sein will, nicht weiter Unrecht tust, verstehe Folgendes in Deinem Sinn

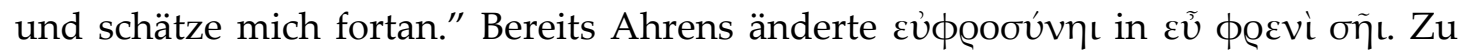

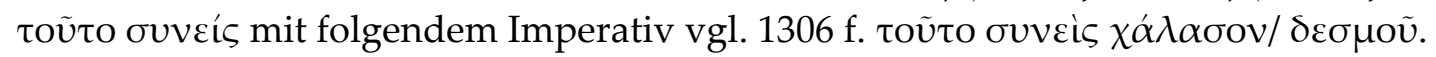

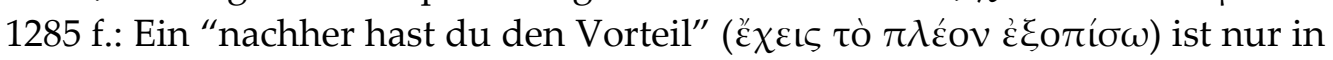
negierter Form sinnvoll. Der Sprecher will dem Knaben klarmachen, daß ihm ein momentan erfolgreicher Betrug auf die Dauer eben keinen Vorteil verschaft. Daher

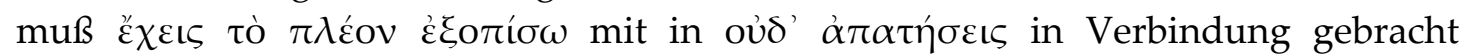

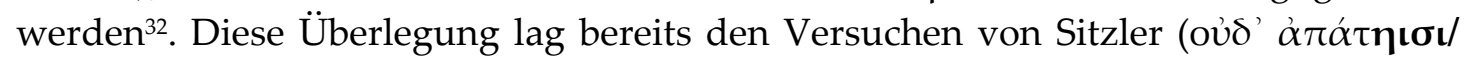

32 Erbse (1998: 238) schreibt nach Emperius oủ statt des Artikels tó, aber dann steht die Negation unnatürlich spät im Satz, und andererseits ist der Artikel sehr berechtigt, da es um einen im Kontext bestimmten Vorteil, nämlich den Erfolg der Täuschung, geht. 


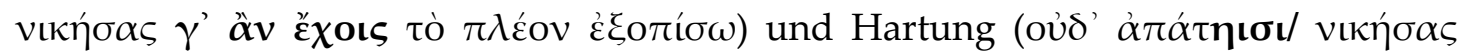

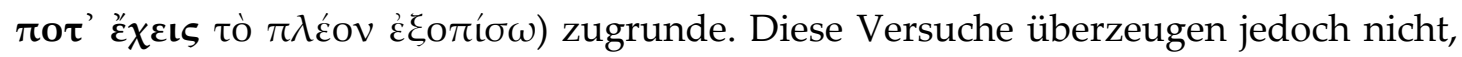
weil man in einer zweistufigen Aussage ("du wirst mich nicht betrügen, und selbst wenn du mit deinem Trug erfolgreich bist, wirst du diesen Vorteil nachher nicht behalten") konzinne Tempora erwarten muß33; das passende Tempus ist naturgemäß

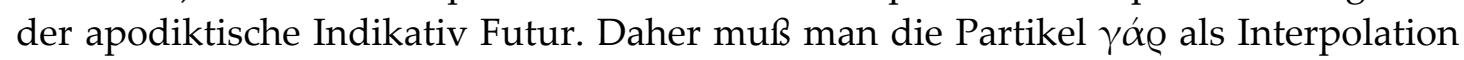
betrachten (erwogen als eine Alternative bei Heimsoeth 1873: 7).

1287 f.: Die Vorstellung eines "Verwundens" ( $ڤ \omega \sigma \omega$, was nach West keine sexuelle Obszoenität enthalten soll) ist unverständlich; bei der von West angeführten Stelle Hyg. fab. 185 ist ausdrücklich von “Töten” die Rede. In der oben dargebotenen

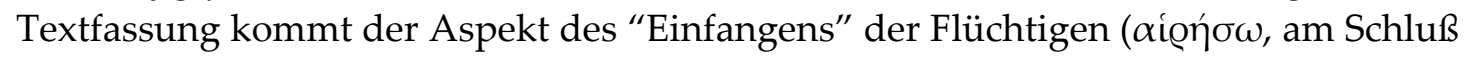

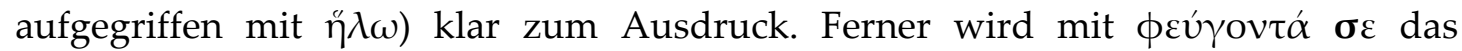
Tertium comparationis klar bezeichnet ${ }^{34}$. Auf der Wirklichkeitsebene werden nur die

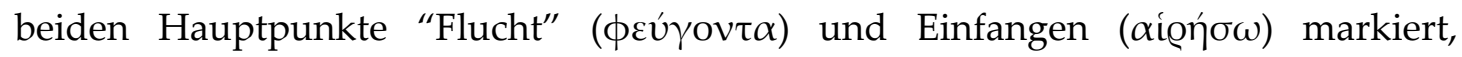

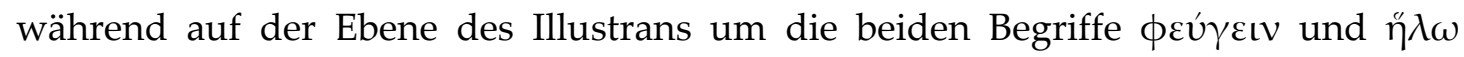
herum eine ausführliche Darstellung angelegt wird. Im Gleichnis wird der in der Haupthandlung nur umrissene Sachverhalt detailliert nachvollzogen.

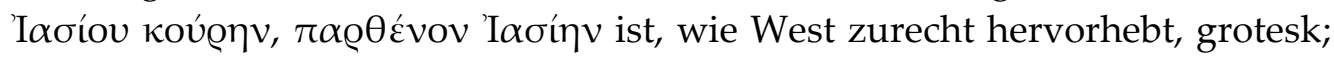
die Verteidigung der Überlieferung durch Renehan (1983: 26) als "a deliberate correction: 'the daughter of Iasius - the unmarried daughter of Iasius'" überzeugt kaum, zumal an keiner der von Renehan angeführten Parallelstellen die beiden sinngleichen patronymischen Bezeichnungen so unmittelbar aufeinanderprallen; ähnliche apologetische Bemühungen bei Koniaris (1984: $105 \mathrm{f}$.), der von einem "artful reversal" spricht. Es handelt sich bei dem inkriminierten Vers aber kaum um eine Schnittstelle zwischen zwei Bearbeitern des Gedichts, sondern nur um einen trivialen

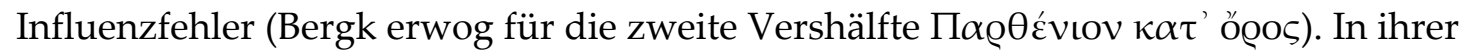

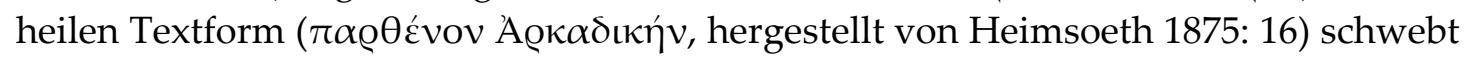
die Stelle wohl im Hero-und-LeanderEpyllion des Musaios vor, wo Atalante ebenfalls als warnendes Beispiel für eine "Flucht" vor der Liebe herangezogen wird (153$155)^{35}$ :

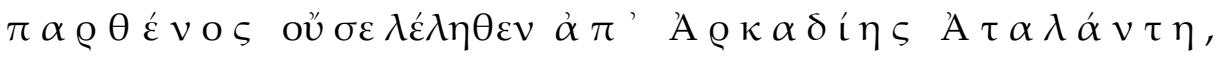

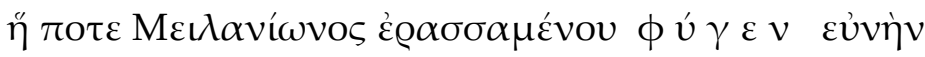

$\pi \alpha \varrho \theta \varepsilon v i ́ n \varsigma \alpha \dot{\alpha} \lambda \varepsilon ́ \gamma o v \sigma \alpha \ldots$

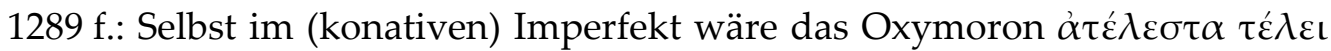
im Rahmen des hier verwendeten Exempels ungeschickt, insofern es die Möglichkeit implizieren könnte, eine Flucht wie die der Atalante bzw. des angesprochenen Knaben könnte gelingen. Formal zieht der metrisch unbegründete Verzicht auf das

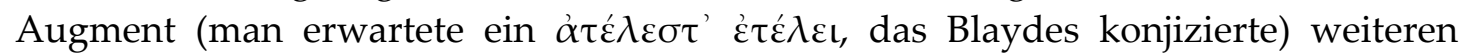

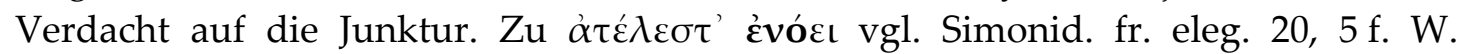

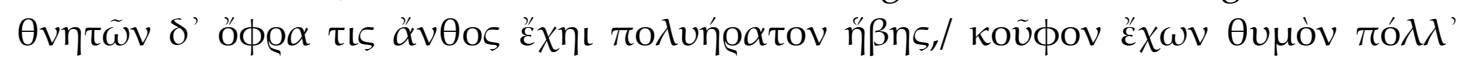
$\dot{\alpha} \tau \dot{\varepsilon} \lambda \varepsilon \sigma \tau \alpha$ v o $\varepsilon \tilde{\imath}$. Entsprechend soll die Flucht der Atalante als nichtiger Gedanke dargestellt werden.

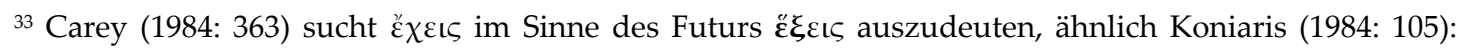
"present of anticipation", vgl. bereits Hudson-Williams zu 1285.

${ }^{34} \mathrm{Zu} \phi \varepsilon v ́ \gamma \varepsilon \iota v$ als Schlüsselbegriff vgl. auch Carey 1984: 364.

${ }^{35}$ Vgl. Rossi 1982: 177-186.
} 
1293 f.: Ein schließliches "Erkennen" ( $\check{\gamma} \gamma \nu \omega$, wohl ohne erotische Eindeutigkeit zu verstehen) der Ehe als Geschenk Aphrodites ist kaum der richtige Gedanke,

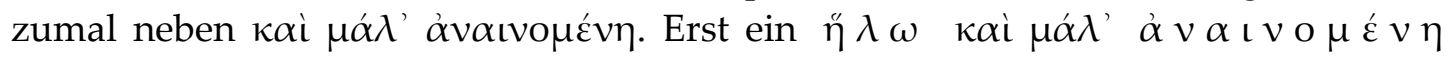

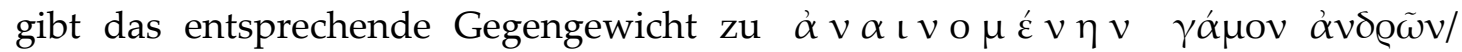
$\phi \varepsilon \dot{v} \gamma \varepsilon \iota v$. Zur erfolglosen Flucht Atalantes vor einer Ehe vgl. auch Ov. met. X 564-566 (Orakel an Atalante): coniuge .../ Nil opus est, Atalanta, tibi: $f u g e$ coniugis usum./ Nec tamen effugies teque ipsavivacarebis. 


\section{b. Übereinstimmungen zwischen den homoerotischen Theognideen und der römischen Liebeselegie}

Die im folgenden vorgetragenen Beobachtungen versuchen zu zeigen, daß bestimmte als gattungsrelevant geltende Characteristica der römischen Liebeselegie (die im allgemeinen auf hellenistische Vorbilder zurückgeführt wird) durchaus frappante Parallelen bereits im Bereich der erotischen Theognideen haben.

Wie in der römischen Liebeselegie verbindet sich auch in den erotischen Epigrammen des Theognis mit der Haltung des Sprechers eine generelle Bevorzugung der Liebe als systematischer Lebensweise, die einen auf Ausübung der Erotik angelegten Lebensstil programmatisch als anderen Lebensformen (ßíoı) überlegen herausstellt.

In einem ersten Epigramm (1253 f. = Solon fr. 23) ${ }^{36}$ erscheint die Knabenliebe als eines von mehreren Kriterien, die einen ö $\lambda \beta \iota \varsigma$ ausmachen:

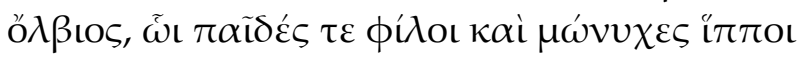

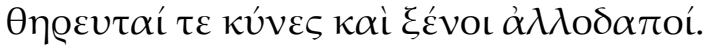

In einer Variation desselben Gedichts (1255 f.) ist die Knabenliebe bereits Ausschlußkriterium für menschliches Glück; wer keine Knaben liebt, kann nicht wirklich glücklich sein:

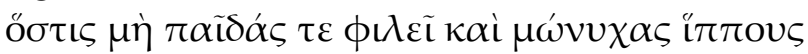

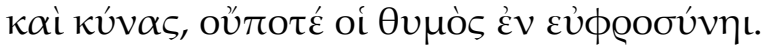

In einem späteren Epigramm (1335 f.) ist die Knabenliebe dann schon einziges Kriterium des ő $\lambda \beta\llcorner$ เo:

ö $\lambda \beta เ$

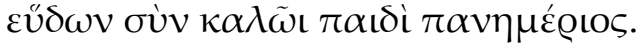

Aus den einzelnen Formulierungen dieses Gedichts geht hervor, daß derjenige, welcher sich in dieser Weise der Knabenliebe verschreibt, nach Hause gehen muß

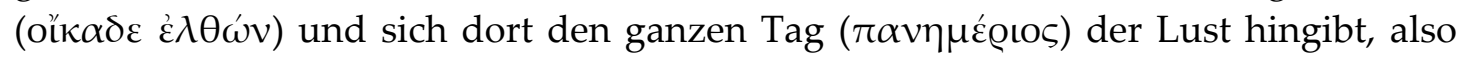
mithin keiner anderen professionellen Tätigkeit nachgehen kann. Diese exklusive Wendung gegen andere Berufe bzw. Lebensformen (ßíos) wird explizit gemacht in

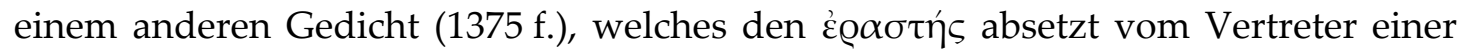
anderen Lebensform, die naturgemäß Abwesenheit von zu Hause und damit Verzicht auf häusliche Erotik impliziert, nämlich derjenigen des Seefahrers:

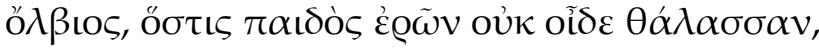

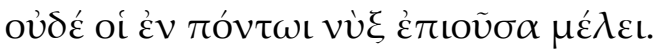

Wie in der römischen Liebeselegie fungiert der um die Gezeiten bekümmerte Seefahrer als kontradiktorisches Gegenbild des sein Heim liebenden Erotikers.

Entsprechend der bösen Kupplerin in der römischen Elegie gibt es auch in den Theognideen Figuren, welche der Liebe entgegenarbeiten, wie aus folgendem Fragment (1278 a/b) hervorgeht:

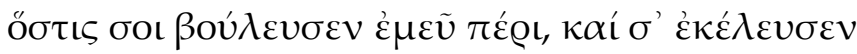

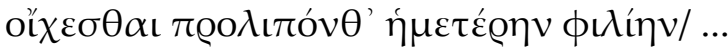

Der Theognideen-Dichter leidet ständig unter dem Gefühl, daß ihm in seinem Liebesverhältnis Unrecht getan wird und er sich trotzdem nicht wehren kann (12791282):

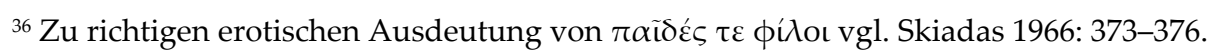




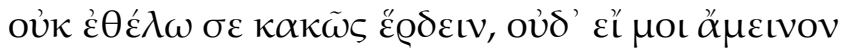

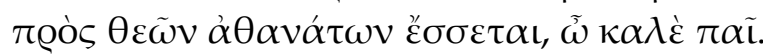

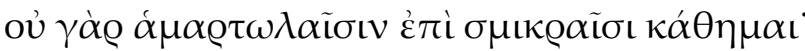

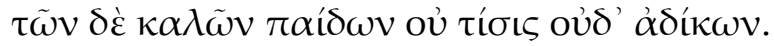

Wie etwa Catull in carm. 76 bittet er die Liebesgottheit darum, die Qual seiner Liebe zu beenden (1323-1325):

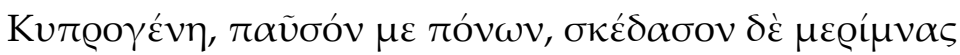

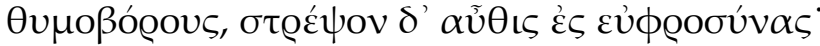

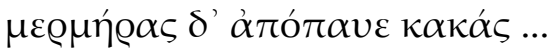

An anderer Stelle wird dieselbe Liebesgottheit entsprechend dem zweiten Elegienbuch Tibulls ${ }^{37}$ als eine im allgemeinen, insbesondere im mythischen Bereich, zerstörerisch wirkende Gottheit begriffen (1231-1234):

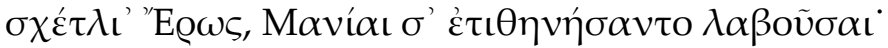

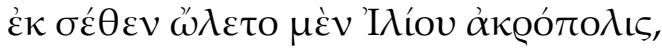

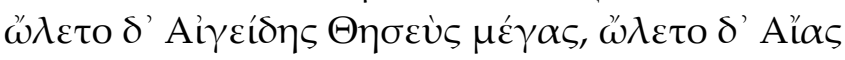

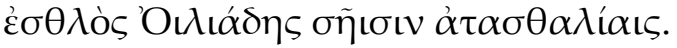

Dieser zerstörerische Eros wird wie bei Tibull (II 1, 67-72 Ipse quoque inter agros interque armenta Cupido/ Natus et indomitas dicitur inter equas:/ Illic indocto primum se exercuit arcu:/ Ei mihi, quam doctas nunc habet ille manus!/ Nec pecudes, velut ante, petit: fixisse puellas/ Gestit et audaces perdomuisse viros) ${ }^{38}$ als eine auf den Menschen bezügliche Sonderausprägung des allgemein-natürlichen Eros gedeutet (1275-1278):

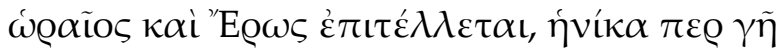

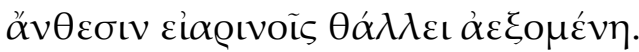

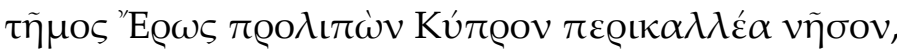

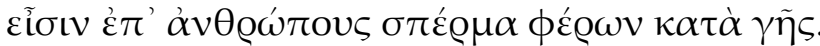

Er macht den Liebenden zu einer in seiner sozialen Umgebung lächerlichen bzw. mitleidheischenden Figur (1107 f. $=1318 \mathrm{ab})$ :

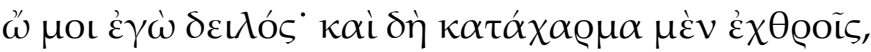

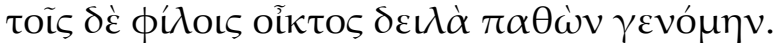

Der Mythos dient bereits in den Theognideen als argumentatives Paradigma, zum einen in der Auseinandersetzung mit dem Geliebten. So wird Atalante herangezogen als ein negatives Exempel dafür, daß man der Liebe nicht entkommen kann (1283-1294):

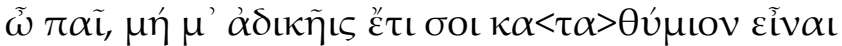

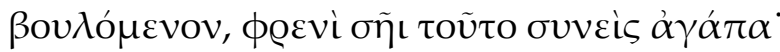

1285

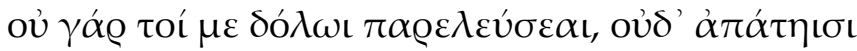

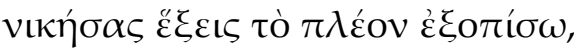

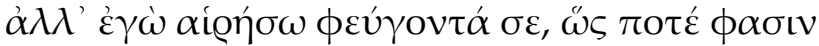

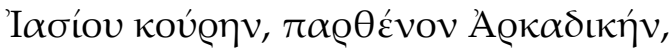

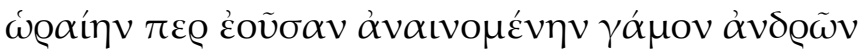

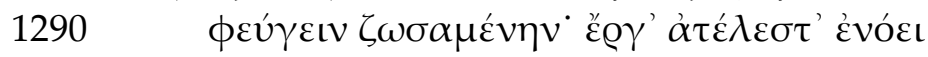

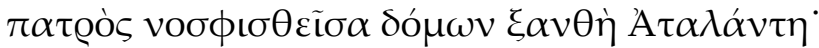

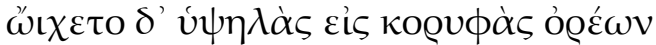

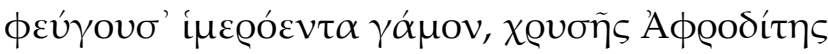

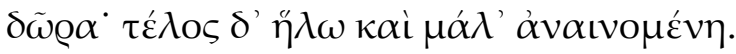

37 Vgl. Gärtner 2003: 218 ff.

${ }^{38}$ Vgl. Gärtner 2003: 219 ff. 
Demgegenüber fungiert Penelope als positives Exempel für die treue Loyalität des Geliebten gegenüber einem lange Zeit abwesenden Liebhaber (1123-1128):

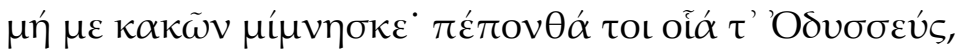

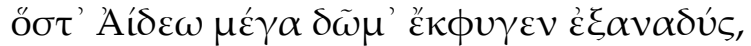

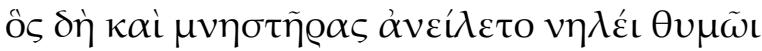

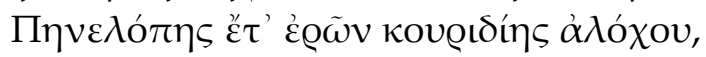

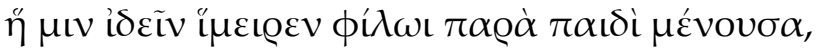

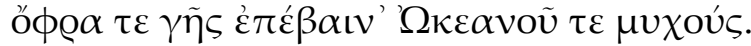

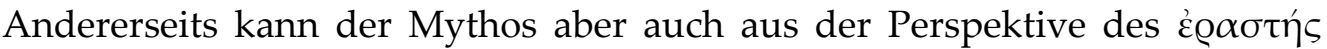
verwendet werden, etwa zur Rechtfertigung der Tatsache homoerotischer Verliebtheit gegenüber einem dritten (1341-1350):

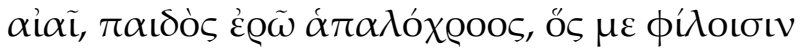

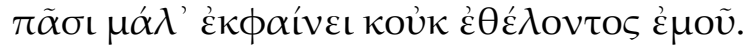

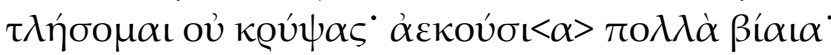

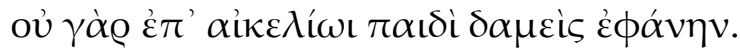

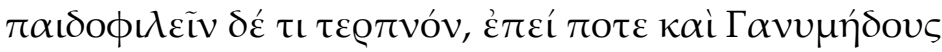

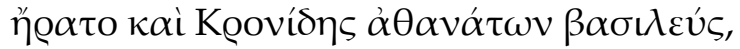

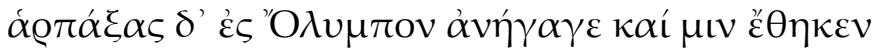

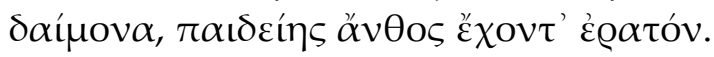

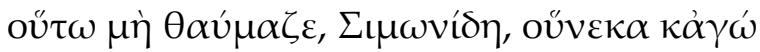

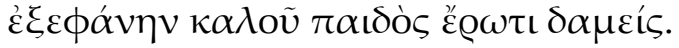

In diesem Fall dient das Exempel also eher der rühmenden Überhöhung des Liebesverhältnisses, im ersten (1283-1294) dagegen der argumentativen Einwirkung auf den Geliebten. Damit sind die beiden bei $\mathrm{Stroh}^{39}$ als für die römische Liebeselegie charakteristisch herausgearbeiteten Kategorien "rühmendes Preisen" und "schmeichelndes Umwerben" zumindest in der rhetorischen Verwendung von Paradigmen in den Theognideen prinzipiell nachweisbar.

Auch ein Bewußtsein von der Funktion der Dichtung als einer argumentativen Einwirkung auf den Geliebten im Sinne Strohs läßt sich in den Theognideen nachweisen. Denn in den Versen 1327-1334, wird man anfangs, um an der Einheit des Gedichts festhalten zu können, nach Orellis Alternativvorschlag $\sigma^{\prime} \alpha i \tau \tilde{\omega} v$, nicht $\sigma^{\prime} \alpha \grave{\imath} v \tilde{\omega} v$ für das überlieferte $\sigma \alpha i ́ v \omega v$ herstellen müssen:

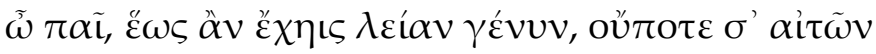

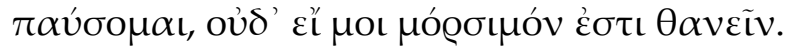

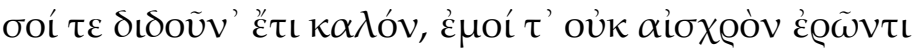

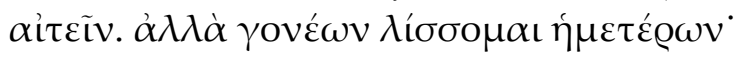

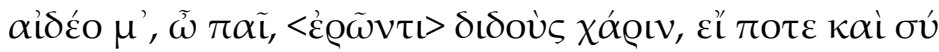

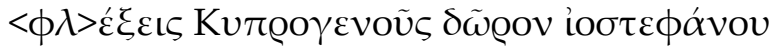

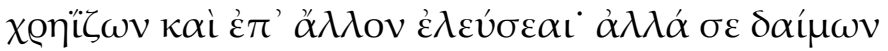

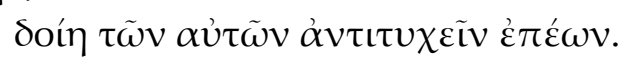

Wenn dieses textkritische Raisonnement zutrifft, ist die ganze Versreihe als ein an den Geliebten gerichtetes Werbegedicht zu verstehen. Dabei begegnet das auch in der römischen Elegie anzutreffende Drohargument, daß der bzw. die

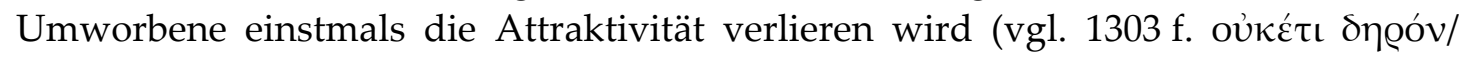

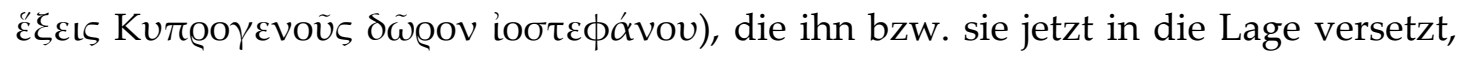

\footnotetext{
${ }^{39}$ Stroh 1971.
} 
hochmütig sein zu können, und sich dann selbst in der verzweifelten Lage befinden

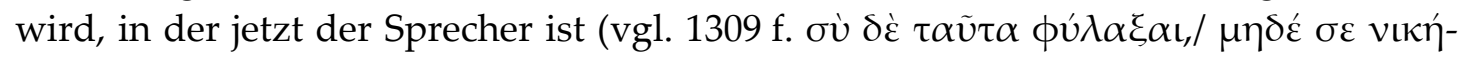

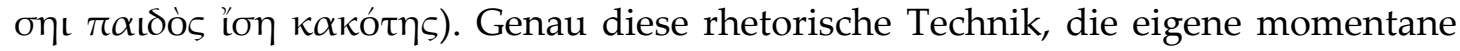
Situation auf den Angesprochenen zu projizieren, liegt auch in den Theognideen vor.

Schließlich läßt sich sogar das bewußte Nebeneinander der beiden auf die eigene Dichtung angewandten Funktionen "rühmendes Preisen" und "schmeichelndes Umwerben" nachweisen, und zwar in dem an Kyrnos gerichteten "Siegelgedicht" (237-254):

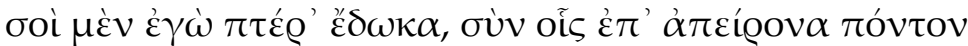

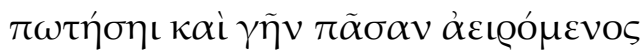

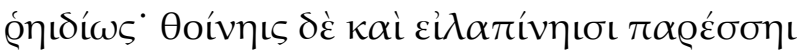

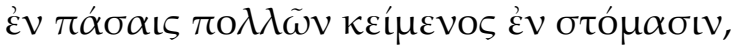

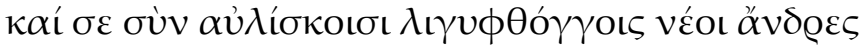

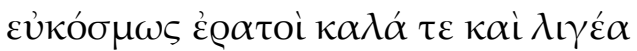

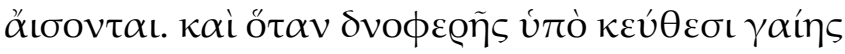

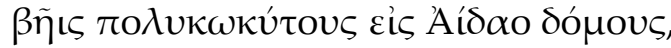

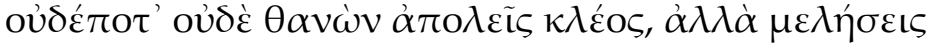

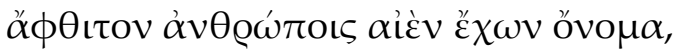

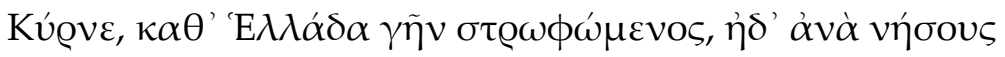

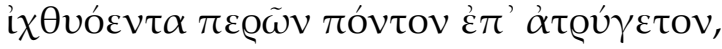

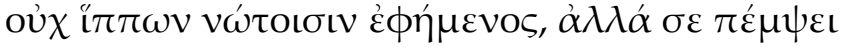

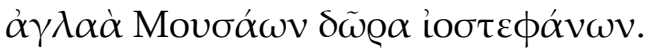

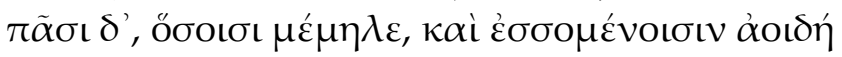

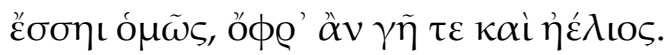

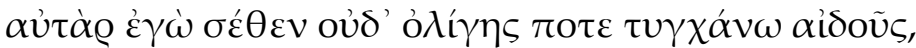

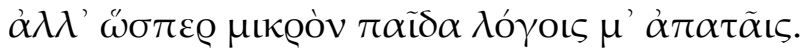

Zunächst malt der Dichten den dem adressierten Knaben aufgrund der Dichtung verheißenen Ruhm breit aus, aber im Schlußdistichon wird deutlich, daß die Dichtung im Sinne ihres Urhebers noch eine ganz andere Funktion hat, nämlich "irgendwann wenigstens eine geringe Respektsbekundung" von dem geliebten Knaben zu erlangen, also seine Gunst einzuwerben. Aber in dieser Hoffnung sieht sich der Dichter, wie Tibull so oft in seinem Nemesis-Buch, getäuscht, und auf diese Enttäuschung reflektiert er innerhalb der in ihrer intendierten Funktion versagenden Verse - wieder wie Tibull (II 4, $13 \mathrm{ff}$. Nec prosunt elegi nec carminis auctor Apollo:/ Illa cava pretium flagitat usque manu./ Ite procul, Musae, si non prodestis amanti:/ .../ .../ .../ Ad dominam faciles aditus per carmina quaero:/ Ite procul, Musae, si nihil ista valent ${ }^{40}$.

Nicht nur die Dichtung soll über das Leben hinaus währen (243 ff.). Wie in der römischen Liebeselegie, wird auch die Liebe im Horizont des Todes vertieft ${ }^{41}$ : Der Dichter stellt sich vor, liebesbedingt (konkret: durch unglückliche Liebe) in die Unterwelt zu gehen, und gibt dem angeredeten Geliebten die Schuld daran (12951298):

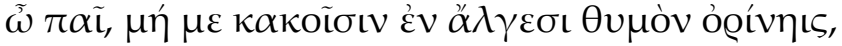

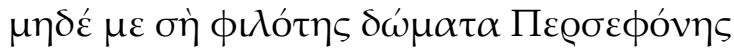

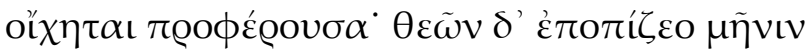

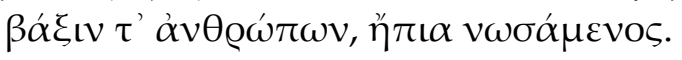

${ }^{40}$ Vgl. Gärtner 2003: 233 f.

${ }^{41}$ Vgl. Gärtner 2003: 235 ff. 
Schließlich zeigt sich auch ein bewußter methodischer Umgang mit dem Phänomen "Liebe", der geradezu die erotodidaktische Lehrdichtung Ovids praefiguriert. In einem textkritisch nicht unproblematischen Gedicht heißt es (1369-1372):

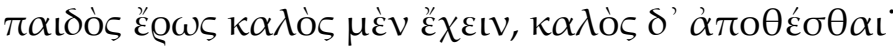

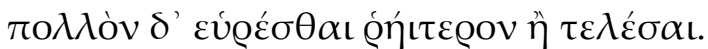

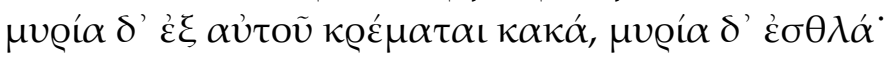

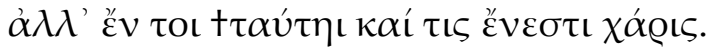

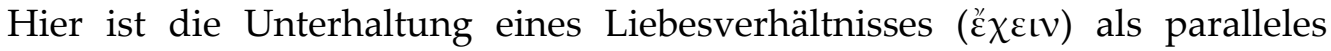
Phänomen zu der "Niederlegung" eines solchen ( $\alpha \dot{\pi} 0 \theta \dot{\varepsilon} \sigma \theta \alpha \mathrm{\iota})$ begriffen. Wenn der

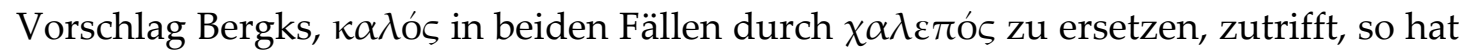
man ein dem ovidischen Vorgehen in Ars amatoria bzw. Remedia amoris genau entsprechendes Verständnis über die Parallelität der beiden Problemfelder "Liebe erlangen" und "Liebe wieder von sich ablegen". Im Pentameter 1370 wird ferner exakt der Grundgedanke artikuliert, welcher der stofflichen Scheidung zwischen erstem und zweitem Buch der Ars amatoria (vgl. ars II 1 ff.) zugrundeliegt, daß

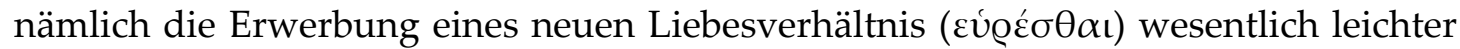
ist als die Vollendung eines bereits bestehenden ( $\tau \varepsilon \lambda \dot{\varepsilon} \sigma \alpha \mathrm{\iota})$.

Priv.-Doz. Dr. Thomas Gärtner

Universität zu Köln, Institut für Altertumskunde

E-Mail: th-gaertner@gmx.de

\section{Literaturverzeichnis}

Allen, T. W. (1930) ,Miscellanea.' - CQ 24, 188-190.

Allen, T. W. (1932) ,Miscellanea.' - CQ 26, 82-87.

Allen, T. W. (1933) ,Miscellanea.' - CQ 27, 51-53.

Allen, T. W. (1940) ,Theognis.' - RPh 66, 211-214.

Carey, C. (1984) ,Theognidea 1283-1294.' - PP 39, 362-366.

Carrière, J. (1954) ,Nouvelles remarques sur l' époque et sur le texte de Théognis.' REG 67, 39-68.

Carrière, J. (1975) ,Quelques corrections et suggestions nouvelles sur le texte de Théognis.' - Pallas 22, 3-11.

Cataudella, Q. (1956) ,Theognidea 903-930.' - RhM 99, 40-46.

Cobet, C. G. (1858) Novae lectiones quibus continentur observationes criticae in scriptores Graecos. Leiden: Brill.

Condello, F. (2001) ,Theognis de amicitia.' - Eikasmos 12, 27-41.

Crönert, W. (1925) ,Kaibel zu Theognis.' - PhW 45, 312-316.

Denniston, J. D. (1954) Greek Particles. 2. ed. Oxford: Clarendon Press.

Erbse, H. (1998) ,Theognidea.' - Hermes 126, 238-241.

Ercolani, A. (1996) ,Theogn. 127.' - MD 37, 195-204.

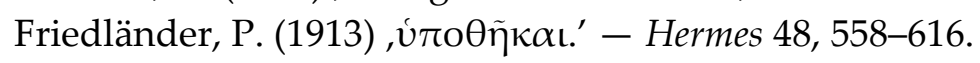

Gärtner, Th. (2003) ,Die Destruktion des elegischen Wertgefüges. Zu den beiden Elegienbüchern des Albius Tibullus.' - Eos 90, 215-245. 
Garzya, A. (1957) ,Note a Teognide.' - Emerita 25, 198-219.

Gerber, D. E. (1970) Euterpe: an anthology of early Greek lyric, elegiac, and iambic poetry. Ed. with introd. remarks and commentary. Amsterdam: Hakkert.

Gerber, D. E. (1991) ,Early Greek elegy and iambus 1921-1989.' - Lustrum 33, 7-225.

Gerber, D. E. (1999) Greek elegiac poetry from the seventh to the fifth centuries BC. Ed. and transl. by D. E. Gerber. (The Loeb classical library; 258.) Cambridge, Mass.: Harvard University Press.

Gianotti, G. F. (1983) ,Theogn. 1299 ss.' - MCr 18, 29-33.

Gomme, A. W. (1925) ,Theognis 959-962.' - CR 39, 101.

Groningen, B. A. van (1966) Theognis: le premier livre. Éd. avec un commentaire. Amsterdam: Noord-Hollandsche Uitgevers Maatschappij.

Haupt, Moriz (1875-1876) Opuscula. I-III. Leipzig: Hirzel.

Heimsoeth, Fr. (1873) Emendationes Theognideae 1. Bonn: Georgi.

Heimsoeth, Fr. (1874) Emendationes Theognideae 2. Bonn: Georgi.

Heimsoeth, Fr. (1875) Emendationes Theognideae 3. Bonn: Georgi.

Herwerden, H. van (1870) Animadversiones philologicae ad Theognidem. Utrecht: Bejers.

Herwerden, H. van (1884) ,Animadversiones ad poetas Graecos.' - Mnemosyne 12, 293-318.

Hudson-Williams, Th. (1910) The elegies of Theognis and other elegies included in the Theognidean sylloge. A revised text based on a new collation of the Mutinensis Ms. London: Bell.

Huijing, E. G. P.; Raalte, M. van (1981) ,Theognidea 197-208.' - Lampas 14, 5-16.

Ijsewijn, J. (1987), Theognidis sententiae a Francisco Craneveldio latine versae (1541).' - Bonanno, A.; Vella, H. C. R. (edd.), Laurea corona. Studies in honour of Edward Coleiro. Amsterdam: Grüner, 14-22.

Kamerbeek, J. C. (1968) ,Notes sur quelques passages de Théognis.' - Mnemosyne 21, 159-162.

Koniaris, G. L. (1984) ,Rez. zu M. Vetta, Theognis. Elegiarum liber secundus.' - AJPh 105, 102-107.

Kotansky, R. (1993) ,P. Berol 21220 = Theognis, Elegiae I, 917-933.' - ZPE 96, 1-5.

Kroll, J. (1955) ,Rez. zur Ausgabe von J. Carriere (Paris 1948).' - Gnomon 27, 76-83.

Kuehner, R.; Gerth, B. (1898) Ausführliche Grammatik der griechischen Sprache. 3. Aufl. Hannover; Leipzig: Hahnsche Buchhandlung.

Leaf, W. (1900-1902) The Iliad. 2. ed. London: Macmillan \& Co.

Leutsch, E. v. (1865) ,Ein Gedicht des Theognis.' - Philologus 22, 17-29.

Luiselli, B. (1959) ,Ad Theognis V. 1202.' - RCCM 1, 394-398.

Madvig, J. N. (1871-1884) Adversaria critica ad scriptores Graecos et Latinos. Hauniae: Gyldendalianae.

Martinelli Tempesta, St. (1998) ,Ancora su Theogn. 127.' - Acme 51, 197-202.

Merkelbach, R. (1956) ,Theognis 127.' - Philologus 100, 133-134.

Mitscherling, J. (1982) ,Xenophon and Plato.' - CQ 76 (n.s. 32), 468-469.

Mühll, Peter von der (1932) ,Zwei griechische Wörter.' - IF 50, 135-139.

Mühll, Peter von der (195) ,Nochmals $\delta \eta v v=$ fern.' - MH 12, 112.

Nagy, G. (1982) ,Theognis of Megara. The poet as seer, pilot, and revenant.' Arethusa 15, 109-128.

Peppmüller, R. (1885) ,Zu Theognis.' - Philologus 44, 235.

Platt, A. (1912) ,Theognidea.' - CR 26, 73-76. 
Pohlenz, M. (1932) ,Rez. zu F. Jacoby, Theognis, Berlin 1931.' - GGA 194, 410-432.

Radermacher, L. (1938) ,Zu griechischen Texten.' - WS 56, 1-2.

Reitzenstein, R. (1893) Epigramm und Skolion: ein Beitrag zur Geschichte der alexandrinischen Dichtung. Giessen: Ricker.

Renehan, R. (1983) ,The early Greek poets. Some interpretations.' - HSPh 87, 1-29.

Rosati, G. (2000) ,Ancora su Teognide in Ovidio.' - SemRom 3, 337-340.

Rossi, M. (1982) ,L'esempio mitico di Atalanta nell' epico Museo.' - Prometheus 8, $177-186$.

Sitzler, J. (1878) Emendationes Theognideae. Baden-Baden.

Sitzler, J. (1885)2 Studien zum Elegiker Theognis. Tauberbischofsheim.

Skiadas, A. D. (1966) ,Bemerkungen zu Solons fr. 13 D.' - Hermes 94, 373-376.

Steffen, V. (1967/8) ,Theognis über die Mischehen.' - Eos 57, 32-36. (= Scripta minora selecta I. Breslau 1973, 106-110.)

Stroh, W. (1971) Die römische Liebeselegie als werbende Dichtung. Amsterdam: Hakkert.

Tarkow, T. A. (1977) ,Theognis 237-254. A reexamination.' - QUCC 26, 99-114.

Vendruscolo, F. (2000) ,Theogn. 126 s.' - Eikasmos 11, 53-58.

Vetta, M. (1972) ,Ps.-Teognide 1231-34 e Apollonio Rodio.' - RFIC 100, 283-294.

Vetta, M. (1980) Theognis. Elegiarum liber secundus. Ed. M. Vetta. Roma: aed. Athenaei.

Wackernagel, J. (1926) Vorlesungen über Syntax I. Basel: Birkhäuser.

West, M. L. (1974) Studies in Greek elegy and iambus. (Untersuchungen zur antiken Literatur und Geschichte; 14.). Berlin; New York: de Gruyter.

Woodbury, L. E. (1991) Collected Writings. Atlanta: Scholars Press.

Xydas, Chr. Ch. (1982) ,Loci lyrici emendandi.' - Parousia 1, 322-328.

Young, D. (ed.) (1961) Theognis. Leipzig: Teubner. 